\title{
1,4-Bis-Dipp/Mes-1,2,4-Triazolylidenes: Carbene Catalysts that Efficiently Overcome Steric Hindrance in the Redox Esterification of $\alpha$ - and $\beta$-Substituted $\alpha, \beta$-Enals
}

Veera Reddy Yatham, Wacharee Harnying, Darius Kootz, Jörg-M. Neudörfl, Nils E. Schlörer and Albrecht Berkessel*

Department of Chemistry, Cologne University, Greinstraße 4, 50939 Cologne, Germany; *e-mail: berkessel@uni-koeln.de

\section{Supporting Information}

Table of contents

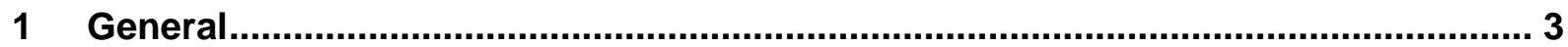

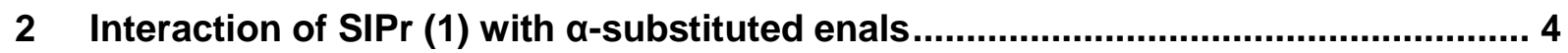

3 Synthesis of mono-Mes/Dipp-substituted triazolium salts...................................... 7

3.1 Synthesis of $N$-(benzylidene)- $N$-phenyl-hydrazine (S1) ............................... 8

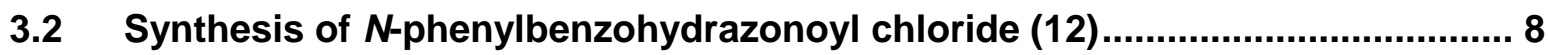

3.3 General procedure (GP) for the synthesis of the mono-Mes/Dippsubstituted hydrazonamide hydrochlorides 14

3.4 General procedure (GP) for the synthesis of the mono-Mes/Dipp-substituted

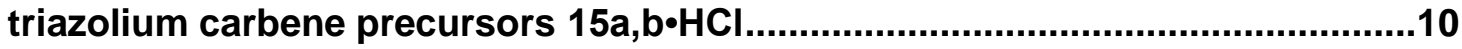

4 Synthesis of bis-Mes/Dipp-substituted triazolium salts ........................................11

4.1 Synthesis of 2,4,6-trimethylphenylhydrazine hydrochloride $(18 a)^{[9]} \ldots \ldots . . . . . . . . .11$

4.2 Synthesis of 2,6-bis(2-propyl)phenylhydrazine hydrochloride (18b)...............12

4.3 Synthesis of (Z)-N-(2,4,6-trimethylphenyl)benzenecarboximidoyl chloride (16a) 13

4.4 Synthesis of (Z)-N-[2,6-di(2-propyl)phenyl]benzenecarboximidoyl chloride (16b)

4.5 Synthesis of 3-phenyl-1,4-bis(2,4,6-trimethylphenyl)-1H-1,2,4-triazol-4-ium chloride $(21 \mathrm{a} \cdot \mathrm{HCl})$

4.6 Synthesis of 1,4-bis[2,6-di(2-propyl)phenyl]-3-phenyl-1H-1,2,4-triazol-4-ium chloride $(21 \mathrm{~b} \cdot \mathrm{HCl})$

5 Synthesis of bis-Ipp/BPh-substituted triazolium salts

5.1 Synthesis of 1,4-bis[2(2-propyl)phenyl]-3-phenyl-1,2,4-triazol-4-ium tetraphenylborate $\left(21 \mathrm{c} \cdot \mathrm{HBPh}_{4}\right)$ 
5.2 Synthesis of 1,4-bis[(2-phenyl)phenyl]-3-phenyl-1,2,4-triazol-4-ium tetraphenylborate $\left(21 \mathrm{~d} \bullet \mathrm{HBPh}_{4}\right)$

6 Synthesis of 2-[2,6-di(2-propyl)phenyl]-6,7-dihydro-5H-pyrrolo[2,1-c][1,2,4]triazol-2-

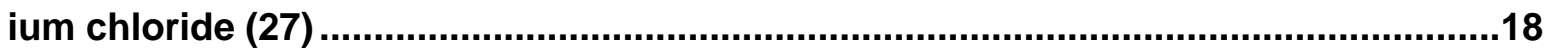

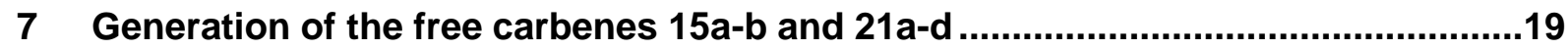

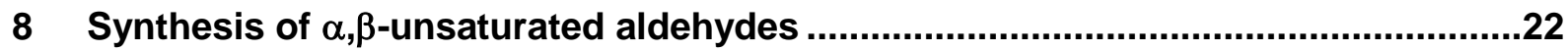

9 General procedure for the redox esterification of enals.........................................26

10 Procedure for the one step synthesis of nepetalactone ....................................33

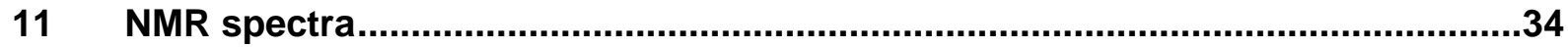

12 X-ray data: Crystal data and structure refinement, selected geometric data, ORTEPs (Oak Ridge Thermal Ellipsoid Plot). ...................................................107

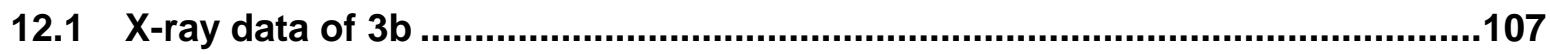

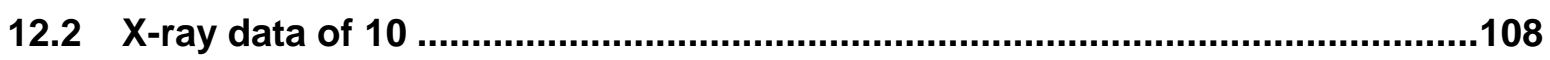

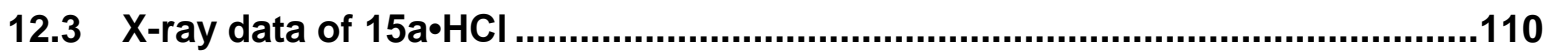

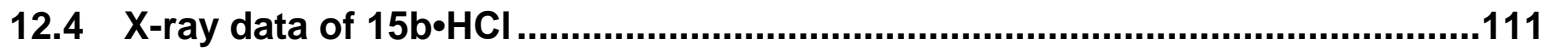

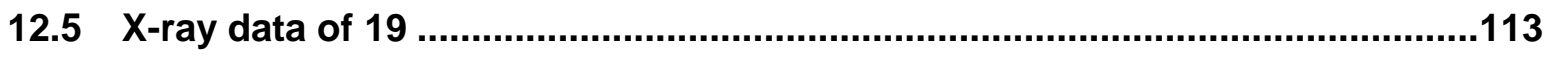

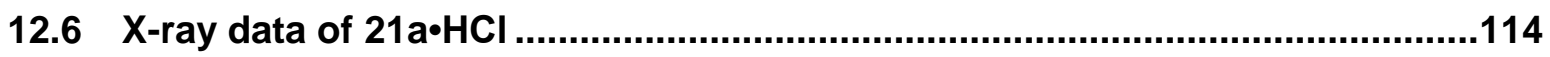

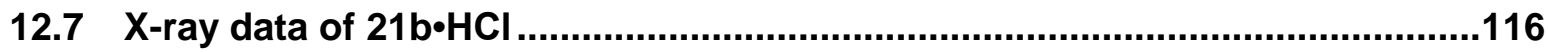

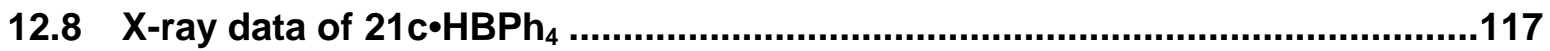

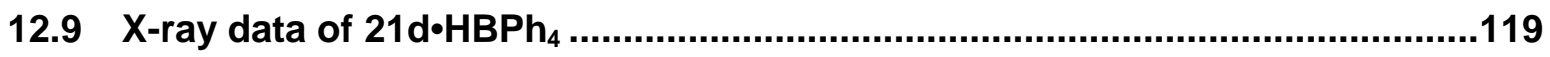

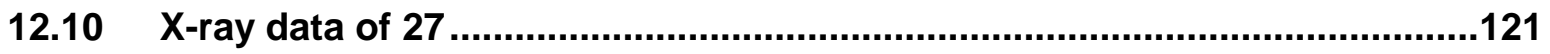

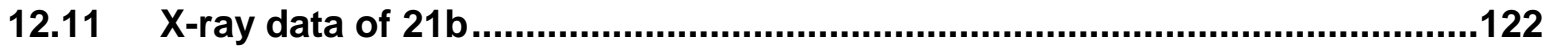

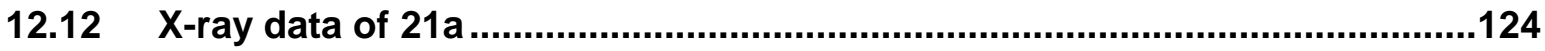

13 References 126 


\section{General}

1,3-Bis(2,6-diisopropylphenyl)imidazolidin-2-ylidene [>98.0\%, SIPr (1)] was purchased from TCI. Methacrolein, ethacrolein, E-2-methyl-2-butenal, E-2-ethyl-2-butenal, E-2-methyl-2pentenal, E-cinnamicaldehyde, $\alpha$-methyl E-cinnamicaldehyde, $n$-butanal, propanal, potassium tert-butoxide, 2,4,6-trimethylaniline, 2,6-bis(2-propyl)aniline, phenylhydrazine, thionyl chloride, triethyl orthoformate, chlorobenzene, $\mathrm{N}$-Chlorosuccinimide, $\mathrm{HCl}$ (4 $\mathrm{M}$ in dioxane), di-tert-butyl dicarbonate $\left(\mathrm{Boc}_{2} \mathrm{O}\right)$, were purchased from Acros Organics. Dimethyl sulfide, E-2-hexenal, 2E,4E-hexadienal, $p$-methoxy $E$-cinnamicaldehyde were purchased from Sigma Aldrich. E-2-Ethylhex-2-enal was provided by BASF and distilled prior to use. Diisopropyl ethyl amine (DIPEA) and DBU were distilled from $\mathrm{KOH}$. Benzyl alcohol was distilled prior to use and stored in a glovebox. THF- $d_{8}$ were passed through neutral aluminium oxide (Brockmann activity 1), degassed by several freeze-pump-thaw cycles and stored over $4 \AA$ molecular sieves in a glovebox. Triazolium salt $11 \cdot \mathrm{HClO}_{4},{ }^{[1]}$ triazolium carbene $11,{ }^{[1]}$ imidazolium salts $30,{ }^{[2]} 36,{ }^{[3]}$ thiazolium salts $28,{ }^{[4]} \mathbf{2 9}{ }^{[5]}, \alpha$-methylene aldehydes 25aa, ${ }^{[25]} 25 \mathbf{a b}^{[25]}$ and $\alpha, \beta$-enals $25 \mathrm{~m}, \mathbf{q}^{[22,23]}$ were synthesized according to the literature procedures cited.

Nuclear magnetic resonance (NMR) spectra were recorded on a Bruker Avance II 600 instrument $\left({ }^{1} \mathrm{H}: 600.20 \mathrm{MHz},{ }^{13} \mathrm{C}: 150.92 \mathrm{MHz}\right)$, on a Bruker AV 500 instrument $(1 \mathrm{H}: 500.13$ $\left.\mathrm{MHz},{ }^{13} \mathrm{C}: 125.76 \mathrm{MHz},{ }^{11} \mathrm{~B}: 160 \mathrm{MHz}\right)$ or on a Bruker AV 300 instrument $\left({ }^{1} \mathrm{H}: 300.13 \mathrm{MHz}\right.$, ${ }^{13} \mathrm{C}: 75.46 \mathrm{MHz},{ }^{19} \mathrm{~F}: 280 \mathrm{MHz}$, ). Spectra were recorded at room temperature unless otherwise stated. Chemical shifts $(\delta)$ are reported in parts per million relative to tetramethylsilane (TMS) or solvent residual signals. The following abbreviations were used for chemical shift multiplicities in ${ }^{1} \mathrm{H}$ NMR spectra: brs = broad singlet, $\mathrm{s}=$ singlet, $\mathrm{d}=$ doublet, $\mathrm{t}=$ triplet, $\mathrm{q}=$ quartet, sept $=$ septet, $\mathrm{m}=$ multiplet. NMR signals were assigned based on of 1D and 2D NMR data $\left({ }^{1} \mathrm{H},{ }^{1} \mathrm{H}\right.$ COSY, ${ }^{1} \mathrm{H},{ }^{1} \mathrm{H}$ NOESY, ${ }^{1} \mathrm{H},{ }^{13} \mathrm{C} \mathrm{HMQC}$, ${ }^{1} \mathrm{H},{ }^{13} \mathrm{C}$ HMBC). IR spectra were recorded on an IR Affinity-1 spectrometer with ATR technique.

HR-MS was performed on a Finnigan MAT 95 (EI) or a Thermo Finnigan LTQ FT mass spectrometer. Melting points were determined on a Büchi apparatus and are uncorrected. Elemental analysis for compounds was conducted using an Elementar Analysesysteme Vario EL instrument. 


\section{Interaction of $\operatorname{SIPr}(1)$ with $\alpha$-substituted enals}

\subsection{Generation of 3-\{1,3-bis[2,6-di(propan-2-yl)phenyl]imidazolidin-2-ylidene\}-2-}

methylpropanal (3a)

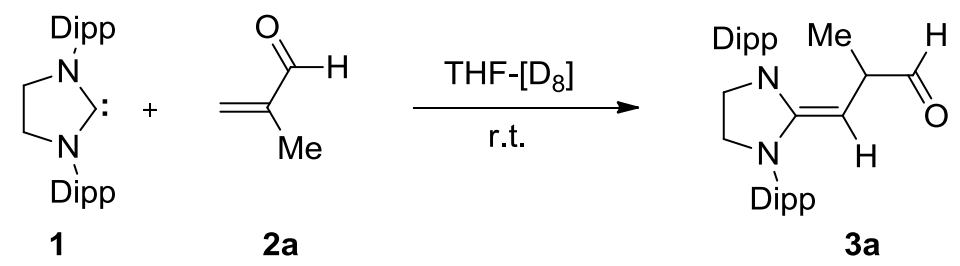

In a glovebox, an NMR tube was charged with SIPr (1) (20 mg, $0.05 \mathrm{mmol}, 1.0$ equiv) in THF- $d_{8}$ and sealed with a septum. Methacrolein (2a) $(4.20 \mu \mathrm{L}, 0.05 \mathrm{mmol}, 1.0$ equiv) was added by means of a syringe and the reaction was followed by NMR spectroscopy.

${ }^{1} \mathrm{H}$ NMR $\left(600 \mathrm{MHz}\right.$, THF- $\left.d_{8}\right): \delta[p p m]=9.1\left(\mathrm{~d},{ }^{3} J_{H H}=0.84 \mathrm{~Hz}, 1 \mathrm{H}, \mathrm{H} 8\right), 7.32-7.28(\mathrm{~m}, 2 \mathrm{H})$,<smiles>CCCCN1CCN(c2c(F)cccc2C(C)C)C(=CC(=O)C=O)c2ccccc21</smiles>
7.25-7.22 $(\mathrm{m}, 4 \mathrm{H}), 3.89-3.77(\mathrm{~m}, 4 \mathrm{H}), 3.57-3.52(\mathrm{~m}, 2 \mathrm{H}), 3.39-3.32(\mathrm{~m}$, $2 \mathrm{H}), 2.37-2.30(\mathrm{~m}, 1 \mathrm{H}), 2.13\left(\mathrm{~d}, 1 \mathrm{H},{ }^{3} J_{H H}=10.6 \mathrm{~Hz} ; 1 \mathrm{H}, \mathrm{H} 6\right), 1.37(\mathrm{~d}, 3 \mathrm{H}$, $\left.{ }^{3} J_{H H}=6.8 \mathrm{~Hz}\right), 1.32-1.30(\mathrm{~m}, 18 \mathrm{H}), 1.25\left(\mathrm{~d}, 3 \mathrm{H},{ }^{3} J_{H H}=6.8 \mathrm{~Hz}\right), 0.63(\mathrm{~d}, 3 \mathrm{H}$, $\left.{ }^{3} J_{H H}=6.6 \mathrm{~Hz}\right) ;{ }^{13} \mathrm{C}$ NMR (150 MHz, THF- $\left.d_{8}\right): \delta[\mathrm{ppm}]=197.9(1 \mathrm{C}, \mathrm{C} 8)$, 150.9 (1C, C2), 149.0, 148.97, 148.34, 147.92, 138.75, 135.9, 128.1, 128.0, 124.23, 124.2, 124.09, 123.94, 65.8 (1C, C6), 51.9, 49.6, 41.8 (1C, C7), 28.23, 28.17, 28.09, 28.04, 25.3, 25.0, 23.94, 23.94, 23.88, 23.77, 22.60, 22.5, 15.0 .

\subsection{Generation of 2-(\{1,3-bis[2,6-di(propan-2-yl)phenyl]imidazolidin-2-ylidene\}me-} thyl)butanal (3b)

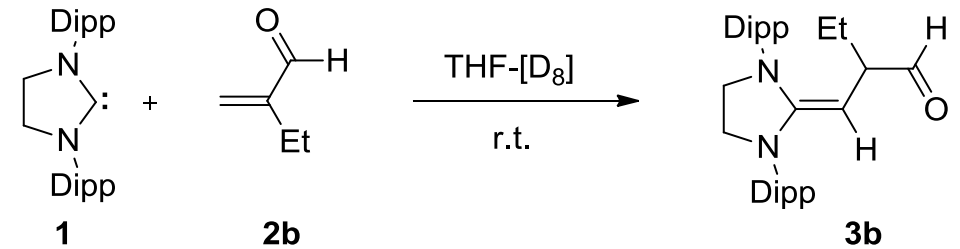

In a glovebox, an NMR tube was charged with $\operatorname{SIPr}(\mathbf{1})(20 \mathrm{mg}, 0.05 \mathrm{mmol}, 1.0$ equiv) in

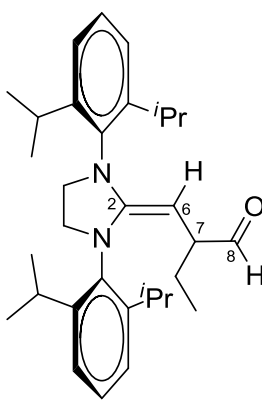

THF- $d_{8}$ and sealed with a septum. Ethacrolein (2b) $(5.0 \mu \mathrm{L}, 0.05 \mathrm{mmol}, 1.0$ equiv) was added by means of a syringe and the reaction was followed by NMR spectroscopy.

${ }^{1} \mathrm{H}$ NMR (600 MHz, THF- $\left.d_{8}\right): \delta[p p m]=9.03\left(\mathrm{~d},{ }^{3} \mathrm{~J}_{\mathrm{HH}}=1.2 \mathrm{~Hz}, 1 \mathrm{H}, \mathrm{H} 8\right)$, 7.34-7.29 (m, $2 \mathrm{H}), 7.26-7.22(\mathrm{~m}, 4 \mathrm{H}), 3.91-3.73(\mathrm{~m}, 4 \mathrm{H}), 3.57-3.51(\mathrm{~m}, 2 \mathrm{H})$, 3.39 (sept, $1 \mathrm{H},{ }^{3} \mathrm{~J}_{H H}=6.8 \mathrm{~Hz}$ ), 3.30 (sept, $\left.1 \mathrm{H},{ }^{3} \mathrm{~J}_{H H}=6.8 \mathrm{~Hz}\right), 2.19-2.17(\mathrm{~m}$, $2 \mathrm{H}, \mathrm{H} 6, \mathrm{H} 7), 1.38\left(\mathrm{~d}, 3 \mathrm{H},{ }^{3} \mathrm{~J}_{H H}=6.8 \mathrm{~Hz}\right), 1.34-1.24(\mathrm{~m}, 20 \mathrm{H}), 1.24(\mathrm{~d}, 3 \mathrm{H}$, $\left.{ }^{3} J_{H H}=6.8 \mathrm{~Hz}\right), 0.46\left(\mathrm{t}, 3 \mathrm{H},{ }^{3} J_{H H}=7.4 \mathrm{~Hz}\right) ;{ }^{13} \mathrm{C}$ NMR $\left(150 \mathrm{MHz}\right.$, THF- $\left.\boldsymbol{d}_{8}\right): \delta[\mathrm{ppm}]=197.5(1 \mathrm{C}$, 
C8), 150.9 (1C, C2), 149.1, 148.9, 148.1, 138.91, 135.9 128.1, 127.9, 124.26, 124.04, 123.8, 64.2 (1C, C6), 51.8, 49.6, 49.1 (1C, C7), 28.23, 28.18, 28.16, 28.06, 25.39, 25.23, 23.96, 23.89, 23.86, 23.78, 23.74, 22.34, 22.11, 11.0 .

\subsection{Generation of (2E)-4-\{1,3-bis[2,6-di(propan-2-yl)phenyl]imidazolidin-2-yl\}-2-} methylbut-2-enal (5a)

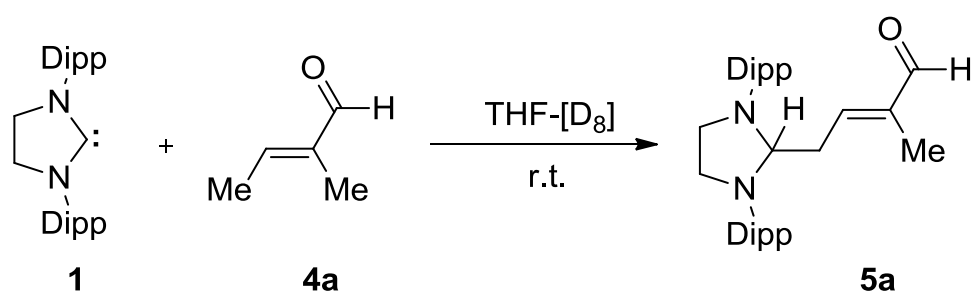

In a glovebox, an NMR tube was charged with $\operatorname{SIPr}(\mathbf{1})(20 \mathrm{mg}, 0.05 \mathrm{mmol}, 1.0$ equiv) in THF- $d_{8}$ and sealed with a septum. E-2-Methyl-2-butenal (4a) $(5.8 \mu \mathrm{L}, 0.05 \mathrm{mmol}, 1.0$ equiv) was added by means of a syringe and the reaction was followed by NMR spectroscopy.

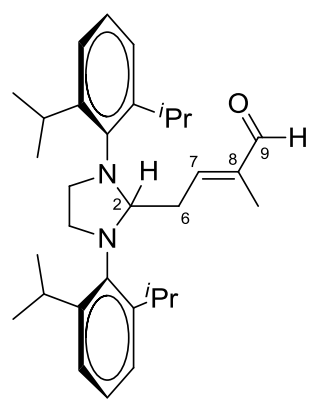

${ }^{1} \mathrm{H}$ NMR $\left(600 \mathrm{MHz}\right.$, THF- $\left.d_{8}\right): \delta[\mathrm{ppm}]=8.78(\mathrm{~s}, 1 \mathrm{H}, \mathrm{H} 9), 7.25-7.24(\mathrm{~m}$, $2 \mathrm{H}), 7.20-7.18(\mathrm{~m}, 4 \mathrm{H}), 5.87\left(\mathrm{t}, 1 \mathrm{H},{ }^{3} J_{H H}=6.7 \mathrm{~Hz}, \mathrm{H} 7\right), 5.0\left(\mathrm{t}, 1 \mathrm{H},{ }^{3} J_{H H}=\right.$ $5.0 \mathrm{~Hz}, \mathrm{H} 2$ ), 3.92 (sept, $2 \mathrm{H},{ }^{3} J_{H H}=6.9 \mathrm{~Hz}$ ), 3.80-3.78 (m, 2H), 3.65$3.64(\mathrm{~m}, 2 \mathrm{H}), 3.48$ (sept, $\left.2 \mathrm{H},{ }^{3} J_{H H}=6.9 \mathrm{~Hz}\right), 2.43-2.41(\mathrm{~m}, 2 \mathrm{H}, \mathrm{H} 6)$, $1.38(\mathrm{~s}, 3 \mathrm{H}), 1.33-1.31(\mathrm{~m}, 12 \mathrm{H}), 1.26-1.25(\mathrm{~m}, 12 \mathrm{H}) ;{ }^{13} \mathrm{C}$ NMR (150 MHz, THF- $\left.d_{8}\right): \delta[p p m]=192.5(1 \mathrm{C}, \mathrm{C} 9), 150.58,149.40(1 \mathrm{C}, \mathrm{C} 7)$, $149.14,139.24$ (1C, C8), 127.26, 124.43, 123.72, 80.45 (1C, C2), 52.17, 35.82 (1C, C6), 28.59, 27.76, 25.46, 24.56, 23.44, 22.71, 8.38.

\subsection{Generation of (2E)-4-\{1,3-bis[2,6-di(propan-2-yl)phenyl]imidazolidin-2-yl\}-2-} ethylbut-2-enal (5b)

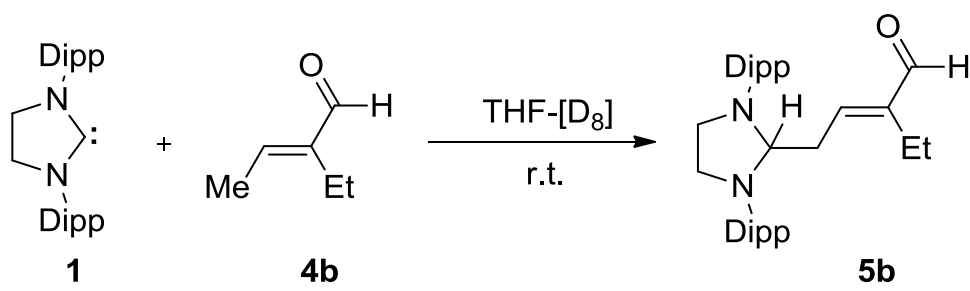

In a glovebox, an NMR tube was charged with $\operatorname{SIPr}(\mathbf{1})(20 \mathrm{mg}, 0.05 \mathrm{mmol}, 1.0$ equiv) in THF- $d_{8}$ and sealed with a septum. E-2-Ethyl-2-butenal (4b) $(5.84 \mu \mathrm{L}, 0.05 \mathrm{mmol}, 1.0$ equiv) was added by means of a syringe and the reaction was followed by NMR spectroscopy.

${ }^{1} \mathrm{H}$ NMR $\left(600 \mathrm{MHz}\right.$, THF- $\left.\boldsymbol{d}_{8}\right): \delta[\mathrm{ppm}]=8.76(\mathrm{~s}, 1 \mathrm{H}, \mathrm{H} 9), 7.27-7.24(\mathrm{~m}, 2 \mathrm{H}, \mathrm{Ar}), 7.20-7.19(\mathrm{~m}$, $4 \mathrm{H}, \mathrm{Ar}$ ), $5.81\left(\mathrm{t}, 1 \mathrm{H},{ }^{3} \mathrm{~J}_{H H}=6.8 \mathrm{~Hz}, \mathrm{H} 7\right), 4.96\left(\mathrm{t}, 1 \mathrm{H},{ }^{3} J_{H H}=4.9 \mathrm{~Hz}, \mathrm{H} 2\right), 3.92$ (sept, $2 \mathrm{H},{ }^{3} J_{H H}=$ $6.9 \mathrm{~Hz}), 3.80-3.78\left(\mathrm{~m}, 2 \mathrm{H}\right.$, ), 3.64-3.63 (m, 2H), 3.47 (sept, $\left.2 \mathrm{H},{ }^{3} \mathrm{~J}_{H H}=6.9 \mathrm{~Hz}\right), 2.47-2.45(\mathrm{~m}$, $2 \mathrm{H}, \mathrm{H} 6), 1.92\left(\mathrm{q},{ }^{3} J_{H H}=7.5 \mathrm{~Hz}, 2 \mathrm{H}\right), 1.33-1.31(\mathrm{~m}, 12 \mathrm{H}), 1.26-1.25(\mathrm{~m}, 12 \mathrm{H}) .0 .65\left(\mathrm{t}, 3 \mathrm{H},{ }^{3} \mathrm{~J}_{H H}\right.$ 
$=7.5 \mathrm{~Hz}) ;{ }^{13} \mathrm{C}$ NMR (150 MHz, THF- $\left.\boldsymbol{d}_{8}\right): \delta[\mathrm{ppm}]=192.5(1 \mathrm{C}, \mathrm{C} 9)$, $150.6,149.23,149.12,145.0,139.3,139.1,127.3,124.4,123.7,80.6$, 52.2 , 35.5, 28.6, 27.8, 25.4, 24.6, 23.4, 22.7, 17.0, 11.8.

2.5 Generation of 3-\{1,3-bis[2,6-di(propan-2-yl)phenyl]imidazolidin-2-ylidene\}-2methylpentanal (7a) and (2E)-4-\{1,3-bis[2,6-di(propan-2-yl)phenyl]imidazolidin-2-yl\}-2methylpent-2-enal (8a)<smiles>Pc1ccccc1</smiles>

1<smiles>C/C=C/CC</smiles>

6a

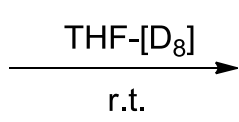

r.t.<smiles>CCC(C=O)=C1N([Pb])CCN1[P+](=O)[O-]</smiles>

$7 a$<smiles>C/C(C=O)=C/C(C)C1([Pb])NCCN1[P+](=O)c1ccccc1</smiles>

$8 a$

In a glovebox, an NMR tube was charged with $\operatorname{SIPr}(1)(20 \mathrm{mg}, 0.05 \mathrm{mmol}, 1.0$ equiv) in THF- $d_{8}$ and sealed with a septum. E-2-Methyl-2-pentenal (6a) $(5.84 \mu \mathrm{L}, 0.05 \mathrm{mmol}, 1.0$ equiv) was added by means of a syringe and the reaction was followed by NMR spectroscopy (see page S44, figure S22-S23 for identification of 7a and 8a).

\subsection{Generation of 3-\{1,3-bis[2,6-di(propan-2-yl)phenyl]imidazolidin-2-ylidene\}-2-} ethylhexanal (7b) and (2E)-4-\{1,3-bis[2,6-di(propan-2-yl)phenyl]imidazolidin-2-yl\}-2ethylhex-2-enal (8b)<smiles>CCC/C=C(\CC)C(=O)OCc1ccccc1</smiles>

1

6b<smiles>CCC(C=O)C(P)=C1N([Pb])CCN1[P+](=O)c1ccccc1</smiles>

7b<smiles></smiles>

8b

In a glovebox, an NMR tube was charged with $\operatorname{SIPr}(\mathbf{1})(20 \mathrm{mg}, 0.05 \mathrm{mmol}, 1.0$ equiv) in THF- $d_{8}$ and sealed with a septum. E-2-Ethyl-2-hexenal (7b) $(7.7 \mu \mathrm{L}, 0.05 \mathrm{mmol}, 1.0$ equiv) was added by means of a syringe and the reaction was followed by NMR spectroscopy (see page 45 , figures S24-S25 for identification of $\mathbf{7 b}$ and $\mathbf{8 b}$ ). 
2.7 Generation of (2E/Z)-4-\{1,3-bis[2,6-di(propan-2-yl)phenyl]imidazolidin-2-yl\}-2methyl-3-phenylbut-2-enal (10)<smiles>Oc1ccccc1</smiles>

1<smiles>C/C(C=O)=C(/C)c1ccccc1</smiles><smiles>CC(C=O)=C(CC1(c2ccccc2)N([Pb])CCN1[Pb])c1ccccc1</smiles>

10

In a glovebox, a NMR tube was charged with SIPr (1) $(20 \mathrm{mg}, 0.05 \mathrm{mmol}, 1.0$ equiv) in THF$d_{8}$ and sealed with a septum. E-Methyl-3-phenylbut-2-enal (9) $(8.2 \mathrm{mg}, 0.05 \mathrm{mmol}, 1.0$ equiv) was added by means of a syringe and the reaction was followed by NMR spectroscopy observed the formation of 10. Similarly when we exposed $E / Z(1: 3)$ mixture of 9 to $\operatorname{SIPr}(\mathbf{1})$, observed the formation of $\mathbf{1 0 .}$<smiles>[2H]C1(CC(=C(C)C=O)c2ccccc2)N2CCN(c3ccccc3)C21P(O)c1ccccc1</smiles>

${ }^{1} \mathrm{H}$ NMR $\left(600 \mathrm{MHz}\right.$, THF- $\left.\boldsymbol{d}_{8}\right): \delta[\mathrm{ppm}]=9.05(\mathrm{~s}, 1 \mathrm{H}, \mathrm{H} 9), 7.29-7.21(\mathrm{~m}$, $5 \mathrm{H}), 7.11-7.10(\mathrm{~m}, 3 \mathrm{H}), 6.98-6.95(\mathrm{~m}, 2 \mathrm{H}), 6.16\left(\mathrm{~d}, 2 \mathrm{H},{ }^{3} \mathrm{~J}_{H H}=7.4 \mathrm{~Hz}\right)$, $4.72\left(\mathrm{t}, 1 \mathrm{H},{ }^{3} \mathrm{~J}_{\mathrm{HH}}=5.4 \mathrm{~Hz}, \mathrm{H} 2\right), 3.83-3.76(\mathrm{~m}, 4 \mathrm{H}), 3.48-3.45(\mathrm{~m}, 2 \mathrm{H})$, 3.15-3.13 (m, 3H), $2.91\left(\mathrm{~d},{ }^{3} \mathrm{~J}_{H H}=5.4 \mathrm{~Hz}, 2 \mathrm{H}, \mathrm{H} 6\right), 1.90(\mathrm{~s}, 3 \mathrm{H}), 1.32(\mathrm{~d}$, $\left.6 \mathrm{H},{ }^{3} J_{H H}=6.8 \mathrm{~Hz}\right), 1.28\left(\mathrm{~d}, 6 \mathrm{H},{ }^{3} J_{H H}=6.8 \mathrm{~Hz}\right), 1.14\left(\mathrm{~d}, 6 \mathrm{H},{ }^{3} J_{H H}=6.8 \mathrm{~Hz}\right), 0.9\left(\mathrm{~d}, 6 \mathrm{H},{ }^{3} J_{H H}=\right.$ $6.0 \mathrm{~Hz}) .{ }^{13} \mathrm{C}$ NMR (150 MHz, THF- $\left.\boldsymbol{d}_{8}\right): \delta[\mathrm{ppm}]=191.5,158.1,150.2,149.1,147.7,142.3$, $141.1,137.1,134.1$, 128.6, 127.5, 127.1, 126.76, 124.2, 124.2, 123.6, 79.2, 52.9, 43.4, 28.1, $28.0,25.5,25.0,23.8,22.5,22.4,10.6$.

\section{Synthesis of mono-Mes/Dipp-substituted triazolium salts}

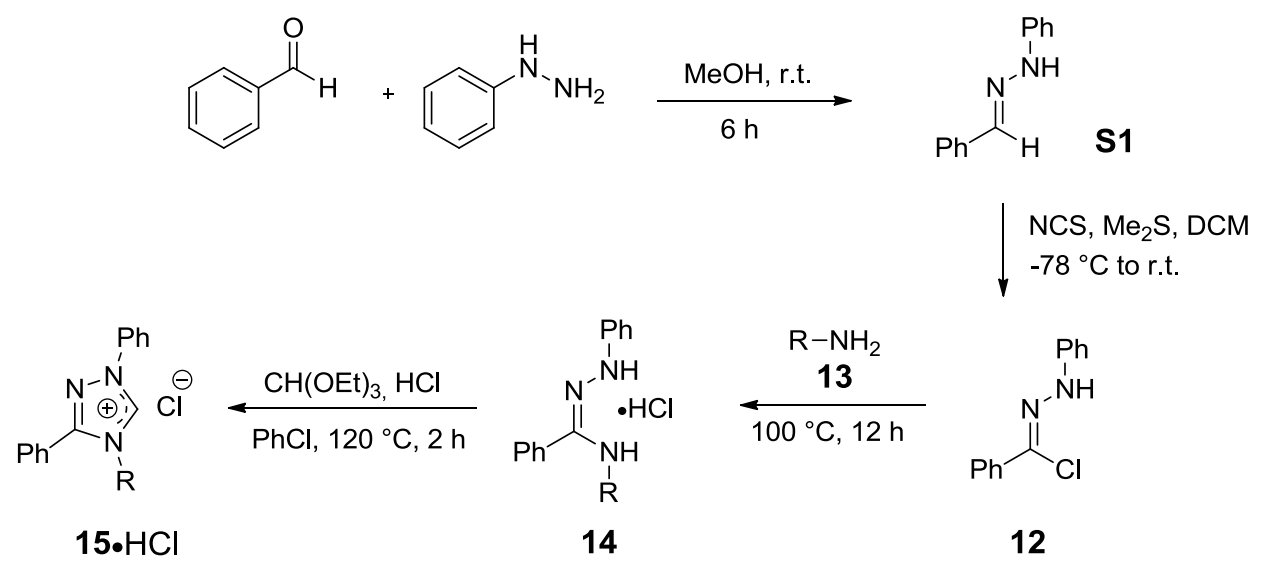




\subsection{Synthesis of $N$-(benzylidene)- $N$ '-phenyl-hydrazine (S1)}<smiles>COCCCCC=NNc1ccccc1</smiles>

S1

To a solution of a phenylhydrazine $(6.4 \mathrm{~g}, 59.4 \mathrm{mmol}, 1.05$ equiv) in $50 \mathrm{~mL}$ of $\mathrm{MeOH}$ was added benzaldehyde ( $6 \mathrm{~g}, 56.5 \mathrm{mmol}, 1.0$ equiv) slowly. The mixture was stirred at room temperature for $6 \mathrm{~h}$. $\mathrm{MeOH}$ was evaporated in vacuo, and the residue was recrystallized from $\mathrm{MeOH}$ to afford $N$-(benzylidene)- $N$ '-phenyl-hydrazine as light brown solid (S1, $7.8 \mathrm{~g}, 70 \%$ yield). The analytical data are consistent with the literature; $;^{[6]} \mathrm{mp} 160-161{ }^{\circ} \mathrm{C}$ (lit. ${ }^{7} 158{ }^{\circ} \mathrm{C}$ ); ${ }^{1} \mathrm{H}$ NMR (600 MHz, $\left.\mathbf{C D C l}_{3}\right): \delta[\mathrm{ppm}]=7.71-7.67(\mathrm{~m}, 3 \mathrm{H}), 7.61(\mathrm{br}, 1 \mathrm{H}), 7.43-7.40(\mathrm{~m}, 2 \mathrm{H})$, 7.36-7.31 (m, $3 \mathrm{H}), 7.17-7.15(\mathrm{~m}, 2 \mathrm{H}), 6.94-6.92(\mathrm{~m}, 1 \mathrm{H}) ;{ }^{13} \mathbf{C}$ NMR (150 MHz, $\left.\mathbf{C D C l}_{3}\right): \delta$ $[p p m]=144.7,137.3,135.3,129.4,128.7,128.5,126.2,120.1,112.8$.

\subsection{Synthesis of $N$-phenylbenzohydrazonoyl chloride (12)}<smiles>c1ccccc1</smiles>

S1<smiles>Cl/C(=N\Nc1ccccc1)c1ccccc1</smiles>

12

To a solution of NCS $\left(8.2 \mathrm{~g}, 61.1 \mathrm{mmol}, 3.0\right.$ equiv) in $\mathrm{CH}_{2} \mathrm{Cl}_{2}(100 \mathrm{~mL})$ at $0{ }^{\circ} \mathrm{C}$ was added dimethyl sulfide ( $9 \mathrm{~mL}, 122 \mathrm{mmol}, 6.0$ equiv) over 5 minutes. After stirring for 15 minutes, the reaction was further cooled to $-78{ }^{\circ} \mathrm{C}$. Then a solution of $\mathrm{N}$-(benzylidene)- $N$ '-phenylhydrazine (S1, $4 \mathrm{~g}, 20.4 \mathrm{mmol}, 1.0$ equiv) in $\mathrm{CH}_{2} \mathrm{Cl}_{2}(70 \mathrm{~mL})$ was added. The reaction was allowed to stir at $-78{ }^{\circ} \mathrm{C}$ for $1 \mathrm{~h}$, then slowly warmed up to room temperature over $3 \mathrm{~h}$. The reaction was quenched by addition of $100 \mathrm{~mL}$ of cold water. The organic layer was then washed with $100 \mathrm{~mL}$ cold water, $50 \mathrm{~mL}$ brine, $50 \mathrm{~mL}$ sat. aq. $\mathrm{Na}_{2} \mathrm{SO}_{3}$, and $100 \mathrm{~mL}$ water. The organic layer was dried over $\mathrm{MgSO}_{4}$, filtered, and concentrated. The crude product was purified by flash column silica gel chromatography to give $\mathrm{N}$-phenylbenzohydrazonoyl chloride as light yellow solid (12, $2.82 \mathrm{~g}, 60 \%$ yield). The analytical data are consistent with the literature; ${ }^{[8]} \mathrm{mp} 127-128^{\circ} \mathrm{C}\left(\right.$ Lit. $\left.^{8}{ }^{127-128}{ }^{\circ} \mathrm{C}\right) ;{ }^{1} \mathbf{H}$ NMR $\left(600 \mathrm{MHz}, \mathrm{CDCl}_{3}\right): \delta[p p m]=8.08$ (brs, $1 \mathrm{H}), 7.98-7.95(\mathrm{~m}, 2 \mathrm{H}), 7.47-7.33(\mathrm{~m}, 5 \mathrm{H}), 7.23-7.21(\mathrm{~m}, 2 \mathrm{H}), 7.0-6.96(\mathrm{~m}, 1 \mathrm{H}) ;{ }^{13} \mathrm{C}$ NMR (600 MHz, $\left.\mathbf{C D C l}_{3}\right): \delta[\mathrm{ppm}]=143.4,134.5,129.4,129.2,128.4,126.4,124.7,121.2$, 113.5; IR (ATR): $\tilde{v}\left[\mathrm{~cm}^{-1}\right]=3304,1595,1581,1571,1502,1487,1446,1435,1313,1267$, 1230, 1134, 1070, 941, 835, 752. 


\subsection{General procedure (GP) for the synthesis of the mono-Mes/Dipp-}

substituted hydrazonamide hydrochlorides 14<smiles>Cl/C(=N\Nc1ccccc1)c1ccccc1</smiles>

12

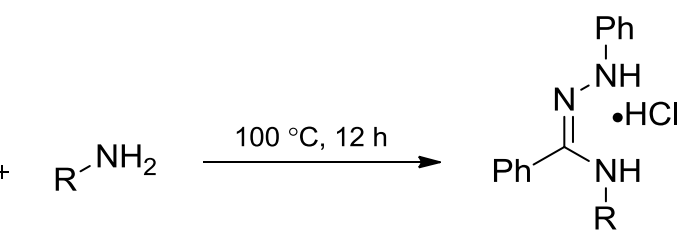

13

14

In a flame-dried round-bottomed flask, $N$-phenylbenzohydrazonoyl chloride (12) (1.0 equiv) was mixed with 2,4,6-trimethylaniline (13a) or 2,6-diisopropylaniline (13b) (1.1 equiv). The reaction mixture was heated to $100{ }^{\circ} \mathrm{C}$ for $12 \mathrm{~h}$. Then the reaction mixture was cooled to r.t. and washed with EtOAc several times, yielding the hydrazonamide hydrochloride 14.

\subsubsection{Synthesis of (Z)-N'-phenyl- $N$-(2,4,6-trimethylphenyl)benzenecarbo-} hydrazonamide hydrochloride (14a)<smiles></smiles>

According to the GP, N-phenylbenzohydrazonoyl chloride (12) $(1.0 \mathrm{~g}, 4.33$ mmol, 1.0 equiv) was reacted with 2,4,6-trimethylaniline (13a, $0.7 \mathrm{~mL}, 4.77$ mmol, 1.1 equiv) to give $14 \mathrm{a}$ as light yellow brown solid (952 $\mathrm{mg}, 60 \%$ yield); ${ }^{1} \mathrm{H}$ NMR $\left(600 \mathrm{MHz} \mathbf{C D C l}_{3}\right): \delta[\mathrm{ppm}]=9.66(\mathrm{~s}, 1 \mathrm{H}), 9.36(\mathrm{~s}, 1 \mathrm{H}), 7.49-7.45$ (m, 3H), 7.30-7.27 (m, 2H), 7.24-7.19 (m, 4H), 6.94-6.92 (m, 1H), 6.79-6.78 (m, 2H), $2.22(\mathrm{~s}$, $3 \mathrm{H}), 2.08(\mathrm{~s}, 6 \mathrm{H}) ;{ }^{13} \mathrm{C}$ NMR (600 MHz, $\left.\mathbf{C D C l}_{3}\right): \delta[\mathrm{ppm}]=164.9,144.9,138.8,134.7,133.6$, 129.8, 129.76, 129.62, 129.59, 129.3, 128.8, 124.1, 121.96, 114.6, 20.9, 18.5; HR-MS (ESI, positive): $m / z$ calcd for $\left[\mathrm{C}_{22} \mathrm{H}_{24} \mathrm{~N}_{3}\right]^{+}[\mathrm{M}]^{+} 330.19647$ found 330.19643 .

\subsubsection{Synthesis of (Z)- $N$-[2,6-di(propan-2-yl)phenyl]- $N$-phenylbenzenecarbo-} hydrazonamide hydrochloride (14b)

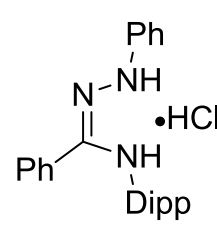
According to the GP, $N$-phenylbenzohydrazonoyl chloride (12) $(2.0 \mathrm{~g}, 8.67$ mmol, 1.0 equiv) was reacted with 2,6-diisopropylaniline (13b, $1.8 \mathrm{ml}, 9.54$ mmol, 1.1 equiv) to give $\mathbf{1 4 b}$ as light yellow brown solid $\left(2.4 \mathrm{~g}, 68 \%\right.$ yield); ${ }^{1} \mathbf{H}$ NMR (600 MHz, $\left.\mathbf{C D C l}_{3}\right): \delta[\mathrm{ppm}]=9.66(\mathrm{~s}, 1 \mathrm{H}), 9.36(\mathrm{~s}, 1 \mathrm{H}), 7.46-7.44(\mathrm{~m}$ $1 \mathrm{H})$, 7.40-7.39 (m, 2H), 7.31-7.23 (m, 8H), 7.09-7.08 (m, 2H), 6.99-6.96 (m, 1H), 2.92 (sept, $\left.2 \mathrm{H},{ }^{3} \mathrm{~J}_{H H}=6.0 \mathrm{~Hz}\right), 1.05\left(\mathrm{~d}, 6 \mathrm{H},{ }^{3} \mathrm{~J}_{H H}=6.0 \mathrm{~Hz}\right), 0.87\left(\mathrm{~d}, 6 \mathrm{H},{ }^{3} J_{H H}=6.0 \mathrm{~Hz}\right) ;{ }^{13} \mathrm{C}$ NMR $(600$ $\left.\mathbf{M H z}, \mathbf{C D C l}_{3}\right): \delta[\mathrm{ppm}]=164.7,145.2,144.9,133.5,129.8,129.6,129.2,128.8,124.3,123.7$, 122.2, 114.9, 28.8, 24.8, 22.0; HR-MS (ESI, positive): $\mathrm{m} / \mathrm{z}$ calcd for $\left[\mathrm{C}_{25} \mathrm{H}_{30} \mathrm{~N}_{3}\right]^{+}[\mathrm{M}]^{+}$ 372.24342 found 372.2431 . 


\subsection{General procedure (GP) for the synthesis of the mono-Mes/Dipp-substituted}

triazolium carbene precursors $15 \mathrm{a}, \mathrm{b} \cdot \mathrm{HCl}$

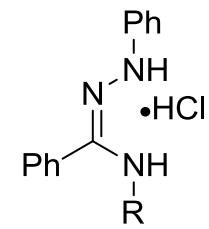

14
$\underset{\mathrm{PhCl}, 120^{\circ} \mathrm{C}, 2 \mathrm{~h}}{\mathrm{CH}(\mathrm{OEt})_{3}, \mathrm{HCl}}$

$15 \cdot \mathrm{HCl}$

A flame-dried round-bottomed flask was charged with hydrazonamide hydrochloride 14 (1.0 equiv), chlorobenzene, triethyl orthoformate (8.0 equiv) and anhydrous $\mathrm{HCl}$ (4 $\mathrm{M}$ in dioxane, 1.0 equiv). The vessel was purged with argon and immersed in a silicon oil bath at $120{ }^{\circ} \mathrm{C}$. The mixture was stirred for $2 \mathrm{~h}$, resulting in a light orange homogeneous solution. The solution was allowed to cool to ambient temperature, and the solvent was removed on a rotary evaporator followed by high vacuum, affording a orange solid. This brown solid was washed with EtOAc several times to yield the triazolium chloride $15 \cdot \mathrm{HCl}$.

\subsubsection{Synthesis of 1,3-diphenyl-4-(2,4,6-trimethylphenyl)-1H-1,2,4-triazol-4-ium chloride $(15 \mathrm{a} \cdot \mathrm{HCl})$}

According to the GP, the hydrazonamide hydrochloride $14 \mathrm{a}$ (500 $\mathrm{mg}, 1.37 \mathrm{mmol}, 1.0$ equiv) in chlorobenzene $(2.0 \mathrm{~mL})$ was reacted with triethyl orthoformate $(1.82 \mathrm{~mL}, 10.93 \mathrm{mmol}, 8.0$ equiv) and anhydrous $\mathrm{HCl}(4 \mathrm{M}$ in dioxane, $0.3 \mathrm{~mL}, 1.36 \mathrm{mmol}, 1.0$ equiv) to give the triazolium chloride $\mathbf{1 5 a} \cdot \mathrm{HCl}$ as light yellow brown solid $(334 \mathrm{mg}, 65 \%) ; \mathrm{mp} 279-280{ }^{\circ} \mathrm{C} ;{ }^{1} \mathbf{H}$

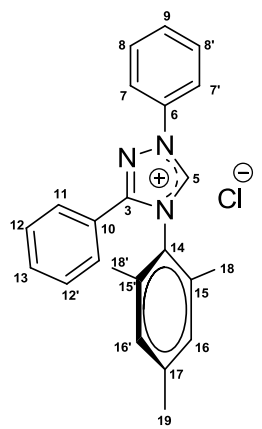

NMR (600 MHz, $\left.\mathrm{CDCl}_{3}\right): \delta[\mathrm{ppm}]=13.64(\mathrm{~s}, 1 \mathrm{H}, \mathrm{H} 5), 8.61-8.60(\mathrm{~m}, 2 \mathrm{H}, \mathrm{H} 7$, $\mathrm{H}^{\prime}$ ), 7.67-7.64 (m, 2H, H8, H8`), 7.59-7.55 (m, 2H, H9, H13), 7.52-7.50 (m, $\left.2 \mathrm{H}, \mathrm{H} 11, \mathrm{H} 11^{\prime}\right), 7.44-7.42$ (m, 2H, H12, H12'), 7.06 (s, 2H, H16, H16'), 2.36 (s, 3H, H19), 2.14 (s, 6H, H18, H18'); ${ }^{13} \mathrm{C}$ NMR (150 MHz, $\mathbf{C D C l}_{3}$ ): $\delta[p p m]$ $=153.4(1 \mathrm{C}, \mathrm{C} 3), 144.0$ (1C, C5), 142.3 (1C, C17), 135.0 (1C, C6), 134.3 (2C, C15, C15'), 132.9 (1C, C13), 130.9 (1C, C9), 130.7 (2C, C16, C16'), 130.3 (2C, C8, C8'), 129.6 (C12, C12‘), 127.9 (1C, C14), 127.8 (2C, C11, C11'), 122.5 (1C, C10), 120.5 (2C, C7, C7'), 21.31 (1C, C19), 18.24 (2C, C18, C18'); HR-MS (ESI, positive): $m / z$ calcd. for $\left[\mathrm{C}_{23} \mathrm{H}_{22} \mathrm{~N}_{3}\right]^{+}[\mathrm{M}]^{+} 340.18082$ found 340.18020 .

\subsubsection{Synthesis of 4-[2,6-di(propan-2-yl)phenyl]-1,3-diphenyl-1 H-1,2,4- triazol-4-ium chloride $(15 \mathrm{~b} \cdot \mathrm{HCl})$}

According to the GP, the hydrazonamide hydrochloride $14 \mathrm{~b}(2.4 \mathrm{~g}, 5.88 \mathrm{mmol}, 1.0$ equiv) in chlorobenzene $(3.0 \mathrm{~mL})$ was reacted with triethyl orthoformate $(7.83 \mathrm{~mL}, 47.1 \mathrm{mmol}, 8.0$ equiv) and anhydrous $\mathrm{HCl}(4 \mathrm{M}$ in dioxane, $1.5 \mathrm{~mL}, 25.1 \mathrm{mmol}, 1.0$ equiv) to give the 
triazolium salt $\mathbf{1 5 b} \cdot \mathrm{HCl}$ as light yellow brown solid (1.9 g, $77 \%)$; mp 257-258 ${ }^{\circ} \mathrm{C} ;{ }^{1} \mathbf{H}$ NMR

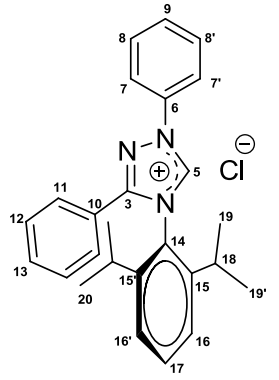

(600 MHz, $\left.\mathrm{CDCl}_{3}\right): \delta[\mathrm{ppm}]=12.1(\mathrm{~s}, 1 \mathrm{H}, \mathrm{H} 5), 8.27-8.25(\mathrm{~m}, 2 \mathrm{H}, \mathrm{H} 7, \mathrm{H} 7$ '), 7.80-7.71(m, 4H, H18, H8', H17, H13), 7.67-7.66 (m, 1H, H19), 7.64-7.54 (m, 4H, H16, H16', H12, H12'), 7.50-7.49 (m, 2H, H11, H11'), 2.66 (sept, $\left.2 \mathrm{H},{ }^{3} J_{H H}=6.0 \mathrm{~Hz}, \mathrm{H} 18, \mathrm{H} 18^{\prime}\right), 1.2\left(\mathrm{~d}, 6 \mathrm{H},{ }^{3} \mathrm{~J}_{H H}=6.0 \mathrm{~Hz}, \mathrm{H} 19, \mathrm{H} 19^{\prime}\right), 0.9$ (d, $\left.6 \mathrm{H},{ }^{3} \mathrm{~J}_{\mathrm{HH}}=6.0 \mathrm{~Hz}, \mathrm{H} 20, \mathrm{H} 20^{\circ}\right) ;{ }^{13} \mathrm{C}$ NMR (600 MHz, $\left.\mathbf{C D C l}_{3}\right): \delta[\mathrm{ppm}]=$ 153.7 (1C, C3), 145.8 (2C, H15, H15), 143.7 (1C, C5), 135.7 (1C, C6), 133.4 (1C, C13),133.3 (1C, C17), 131.3 (1C, C9), 130.5 (1C, C8, C8'), 130.0 (2C, C12, C12'), 128.6 (2C, C11, C11'), 127.6 (1C, C14), 126.1 (2C, C16, C16'), 122.6 (1C, C10), 121.4 (2C, C7, C7'), 28.4 (2C, C18, C18'), 25.3 (C19, C19'), 22.9 (C20, C20'); HR-MS (ESI, positive): $\mathrm{m} / z$ calcd for $\left[\mathrm{C}_{26} \mathrm{H}_{28} \mathrm{~N}_{3}\right]^{+}[\mathrm{M}]^{+} 382.22777$ found 382.22734 .

\section{Synthesis of bis-Mes/Dipp-substituted triazolium salts}

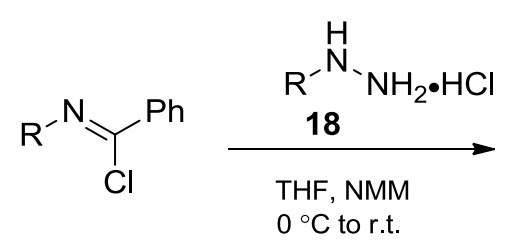

16

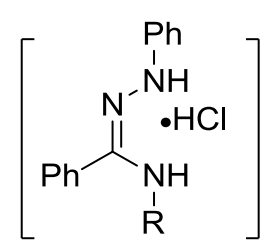

20

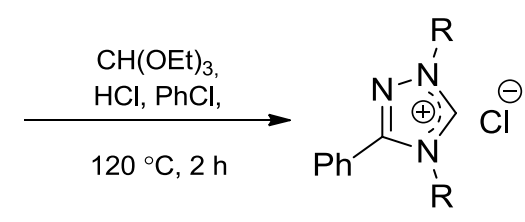

21a

\subsection{Synthesis of 2,4,6-trimethylphenylhydrazine hydrochloride $(18 a)^{[9]}$}

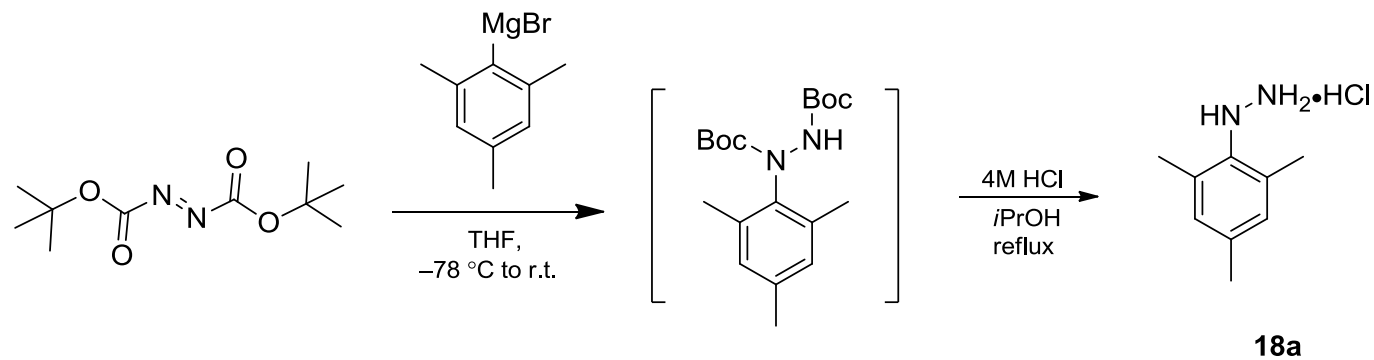

To a stirred solution of di-tert-butyl azodicarboxylate $(5.0 \mathrm{~g}, 21.71 \mathrm{mmol}, 1.0$ equiv. $)$ in THF $(80 \mathrm{~mL})$ was added 2-mesitylmagnesium bromide (1.0 $\mathrm{M}$ in $\left.\mathrm{Et}_{2} \mathrm{O}, 22 \mathrm{~mL}, 22 \mathrm{mmol}\right)$ at $-78^{\circ} \mathrm{C}$. The resulting solution was stirred at $-78{ }^{\circ} \mathrm{C}$ for 1 hour and then quenched with acetic acid (1.3 $\mathrm{mL}, 22.8 \mathrm{mmol}, 1.05$ equiv). The mixture was allowed to warm to r.t. and $\mathrm{H}_{2} \mathrm{O}(20 \mathrm{~mL})$ was added. After extraction with $\mathrm{Et}_{2} \mathrm{O}$, the combined organic layers were washed with brine $(50 \mathrm{~mL})$, dried with anh. $\mathrm{MgSO}_{4}$, filtered and concentrated in vacuo to give the crude product, as light yellow oil, which was used directly in the next step.

The above crude product was dissolved in $i-\mathrm{PrOH}(45 \mathrm{~mL})$ and $\mathrm{HCl}(4 \mathrm{M}$ in dioxane, $16 \mathrm{~mL}, 65 \mathrm{mmol}$ ) was added. The mixture was heated to reflux for $30 \mathrm{~min}$ and then cooled to 
$0{ }^{\circ} \mathrm{C}$. $\mathrm{Et}_{2} \mathrm{O}(50 \mathrm{~mL})$ was added, resulting in a solid precipitating. The precipitate was collected by suction filtration, washed with $\mathrm{Et}_{2} \mathrm{O}$ and dried in high vacuo for several hours to give the mesitylhydrazine hydrochloride $18 \mathrm{a}$ as an off-white solid (2.43 $\mathrm{g}, 60 \%)$. The analytical data are consistent with the literature: ${ }^{[9]} \mathrm{mp} 194{ }^{\circ} \mathrm{C}$ ( Lit. $^{9}{ }^{192-194}{ }^{\circ} \mathrm{C}$ ); ${ }^{1} \mathbf{H}$ NMR $\left(600 \mathrm{MHz}, \mathrm{CDCl}_{3}\right): \delta[\mathrm{ppm}]=9.76(\mathrm{~s}, 3 \mathrm{H}), 7.50(\mathrm{~s}, 1 \mathrm{H}), 6.87(\mathrm{~s}, 2 \mathrm{H}), 2.35(\mathrm{~s}, 6 \mathrm{H}), 2.20$ (s, $3 \mathrm{H}) ;{ }^{13} \mathrm{C}$ NMR (150 MHz, $\mathrm{CDCl}_{3}$ ): $\delta[\mathrm{ppm}]=137.9,136.1,134.8,129.0,39.4,20.4,17.8$.

\subsection{Synthesis of 2,6-bis(2-propyl)phenylhydrazine hydrochloride (18b)}

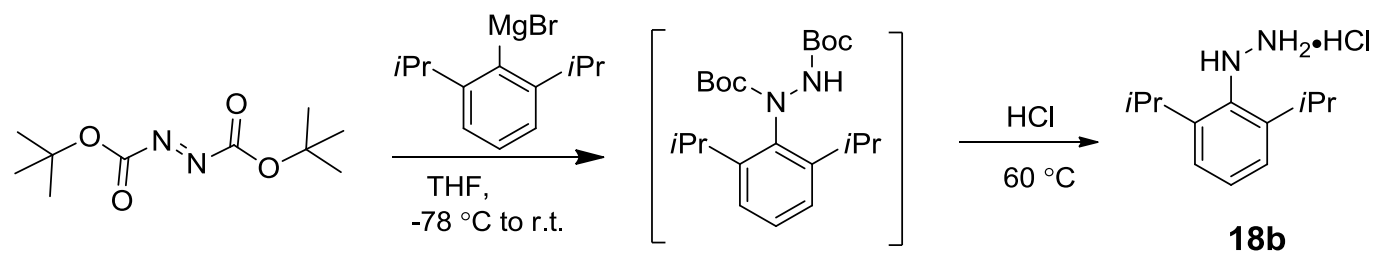

A mixture of 2-bromo-1,3-diisopropylbenzene (2.5 g, $10.4 \mathrm{mmol})$, magnesium (265 mg, 10.8 $\mathrm{mmol}$ ), tetrahydrofuran $\left(15 \mathrm{~mL}\right.$ ), and a trace of iodine was heated to $65{ }^{\circ} \mathrm{C}$ (oil bath temperature) for $3 \mathrm{~h}$ and then cooled to room temperature. The resulting Grignard reagent ${ }^{[10]}$ was added to a stirred solution of di-tert-butyl azodicarboxylate $(2.39 \mathrm{~g}, 10.37 \mathrm{mmol}, 1.0$ equiv) in THF (40 mL) at $-78^{\circ} \mathrm{C}$. The reaction mixture was stirred at $-78^{\circ} \mathrm{C}$ for $1 \mathrm{~h}$ and then quenched with acetic acid $(0.6 \mathrm{~mL}, 11.40 \mathrm{mmol}, 1.1$ equiv). The mixture was allowed to warm to r.t. and $\mathrm{H}_{2} \mathrm{O}(10 \mathrm{~mL})$ was added. The mixture was extracted with $\mathrm{Et}_{2} \mathrm{O}(2 \times 50 \mathrm{~mL})$ and the combined organic layers were washed with brine $(20 \mathrm{~mL})$, dried, filtered and concentrated in vacuo to give the crude product, which was used directly in the next step.

The above crude product was dissolved in $i-\mathrm{PrOH}(15 \mathrm{~mL})$ and $\mathrm{HCl}(4 \mathrm{M}$ in dioxane, $14 \mathrm{~mL}, 57 \mathrm{mmol}$ ) was added. The mixture was heated to reflux for $30 \mathrm{~min}$, cooled to $0{ }^{\circ} \mathrm{C}$ and diluted with $\mathrm{Et}_{2} \mathrm{O}(25 \mathrm{~mL})$. The resulting mixture was kept in the freezer $\left(0-5{ }^{\circ} \mathrm{C}\right)$ for $1 \mathrm{~h}$, resulting in a white solid precipitation. The solid was collected by suction filtration and washed with cold $\mathrm{Et}_{2} \mathrm{O}$ to give $\mathbf{1 8 b}$ as a light brown solid $\left(1.0 \mathrm{~g}, 42 \%\right.$ yield): ${ }^{1} \mathbf{H}$ NMR (600 MHz, DMSO-d $\left.\mathrm{d}_{6}\right): \delta[\mathrm{ppm}]=9.75(\mathrm{~s}, 3 \mathrm{H}), 7.31-7.26(\mathrm{~m}, 1 \mathrm{H}), 7.18-7.15(\mathrm{~m}, 2 \mathrm{H}), 5.91(\mathrm{~s}, 1 \mathrm{H})$, 3.41 (sept, $2 \mathrm{H},{ }^{3} \mathrm{~J}_{H H}=6.75 \mathrm{~Hz}$ ), $1.23\left(\mathrm{~d}, 12 \mathrm{H},{ }^{3} \mathrm{~J}_{H H}=6.75 \mathrm{~Hz}\right.$ ); ${ }^{13} \mathrm{C}$ NMR (150 MHz, DMSO$\left.\mathbf{d}_{6}\right): \delta[\mathrm{ppm}]=146.6,138.2,128.6,124.1,27.6,24.6$; IR (ATR): $\tilde{v}\left[\mathrm{~cm}^{-1}\right]=3236,2962,2865$, 1593, 1576, 1525, 1490, 1457, 1379, 1360, 1260, 1114, 1107, 1085, 1057, 1044, 1020, 891, 869, 802, 757, 779; HR-MS (EI): $m / z$ calcd for $\left[\mathrm{C}_{12} \mathrm{H}_{20} \mathrm{~N}_{2}\right]^{+}[\mathrm{M}-\mathrm{HCl}]^{+} 192.1626$ found 192.162 . 
4.3 Synthesis of (Z)-N-(2,4,6-trimethylphenyl)benzenecarboximidoyl chloride (16a)<smiles>C[As](C)(=O)(O)c1ccccc1</smiles>

$\mathrm{N}$-Mesitylbenzamide ( $4 \mathrm{~g}, 16.71 \mathrm{mmol}, 1.0$ equiv) was refluxed in $6.0 \mathrm{ml}$ of $\mathrm{SOCl}_{2}$ (excess) for $2 \mathrm{~h}$ (oil bath at $90^{\circ} \mathrm{C}$ ), after which the bath temperature was raised to $140{ }^{\circ} \mathrm{C}$ and the remaining $\mathrm{SOCl}_{2}$ and traces of $\mathrm{HCl}$ were distilled off to give the imidoyl chloride $16 \mathrm{a}(4.2 \mathrm{~g}$, $98 \%)$ as a slightly yellow liquid. The analytical data were consistent with the literature. ${ }^{[11]}{ }^{1} \mathbf{H}$ NMR (600 MHz, $\left.\mathbf{C D C l}_{3}\right): \delta[p p m]=8.27-8.25(\mathrm{~m}, 2 \mathrm{H}), 7.62-7.60(\mathrm{~m}, 1 \mathrm{H}), 7.55-7.53(\mathrm{~m}, 2 \mathrm{H})$, $6.97(\mathrm{~s}, 2 \mathrm{H}), 2.36(\mathrm{~s}, 3 \mathrm{H}), 2.14(\mathrm{~s}, 6 \mathrm{H}) ;{ }^{13} \mathrm{C} \mathrm{NMR}\left(150 \mathrm{MHz}, \mathbf{C D C l}_{3}\right): \delta[\mathrm{ppm}]=144.0,143.8$, 135.1, 133.7, 132.1, 129.4,128.57, 128.5, 126.3, 20.9, 17.9; IR (ATR): $\tilde{v}\left[\mathrm{~cm}^{-1}\right]=2916$, $1654,1476,1448,1375,1238,1188,1178,1100,885,850,768,734$.

\subsection{Synthesis of (Z)-N-[2,6-di(2-propyl)phenyl]benzenecarboximidoyl chloride (16b)}

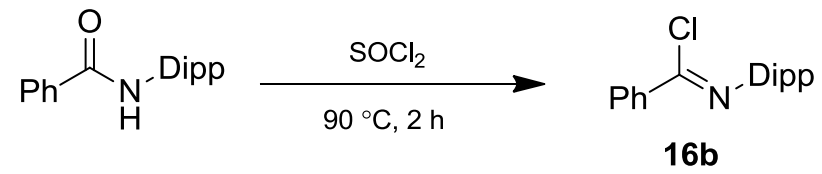

$N$-[2,6-Di(propan-2-yl)phenyl]benzamide $(4 \mathrm{~g}, 16.71 \mathrm{mmol}, 1.0$ equiv) was refluxed in $6.0 \mathrm{ml}$ of $\mathrm{SOCl}_{2}$ (excess) for $2 \mathrm{~h}$ (oil bath at $90^{\circ} \mathrm{C}$ ), after which the bath temperature was raised to $140{ }^{\circ} \mathrm{C}$ and the remaining $\mathrm{SOCl}_{2}$ and traces of $\mathrm{HCl}$ were distilled off. The imidoyl chloride 12b $(4.05,95 \%)$ was obtained as a slightly yellow solid. The analytical data were consistent with the literature; ${ }^{[12]}{ }^{1} \mathbf{H}$ NMR $\left(600 \mathbf{~ M H z}, \mathbf{C D C l}_{3}\right): \delta[p p m]=8.26-8.24(\mathrm{~m}, 2 \mathrm{H}), 7.63-7.60(\mathrm{~m}$, $1 \mathrm{H})$, 7.56-7.53 (m, 2H), 7.23-7.22 (m, 3H), $2.85(\mathrm{~m}, 2 \mathrm{H}), 1.26-1.24(\mathrm{~m}, 6 \mathrm{H}), 1.20-1.19(\mathrm{~m}$, $6 \mathrm{H}) ;{ }^{13} \mathrm{C}$ NMR (150 MHz, $\left.\mathbf{C D C l}_{3}\right): \delta[\mathrm{ppm}]=143.9,143.6,136.7,135.1,132.1,129.5,128.6$, 124.9, 123.1, 28.7, 23.4, 22.9; IR (ATR): $\tilde{v}\left[\mathrm{~cm}^{-1}\right]=2958,1662,1647,1581,1448,1361$, 1338, 1255, 1224, 1166, 891, 794, 758, 686, 634.

\subsection{Synthesis of 3-phenyl-1,4-bis(2,4,6-trimethylphenyl)-1H-1,2,4-triazol-4-ium chloride $(21 \mathrm{a} \cdot \mathrm{HCl})$}

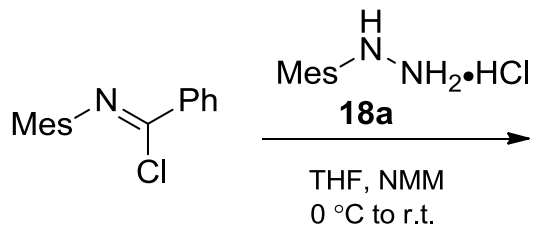

$16 a$

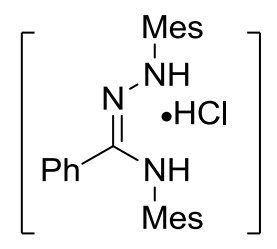

20a

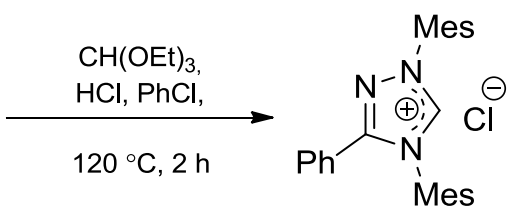

$21 \mathrm{a} \cdot \mathrm{HCl}$ 
A flame-dried round-bottomed flask was charged with the imidoyl chloride $16 \mathrm{a}$ (2.2 $\mathrm{g}$, $8.57 \mathrm{mmol}, 0.8$ equiv), THF $(50 \mathrm{ml})$ and the mesitylhydrazinium hydrochloride $18 \mathrm{a}(2.0 \mathrm{~g}$, $10.71 \mathrm{mmol}, 1.0$ equiv). The reaction mixture was cooled to $0^{\circ} \mathrm{C}$, and $\mathrm{N}$-methylmorpholine (1.2 ml, $10.7 \mathrm{mmol}, 1.0$ equiv) was added. After stirring at $0{ }^{\circ} \mathrm{C}$ for $3 \mathrm{~h}$, the ice bath was removed and stirring was continued overnight. $\mathrm{MeOH}(5 \mathrm{ml})$ was added, and the mixture was stirred for another $30 \mathrm{~min}$. The solvent was removed under reduced pressure at $20^{\circ} \mathrm{C}$. Water $(100 \mathrm{ml})$ was added, and the mixture was extracted with DCM $(500 \mathrm{ml})$ to afford the hydrazonamide hydrochloride $20 \mathrm{a}(3.0 \mathrm{~g}, 68 \%$ yield), which was used in the next step without further purification.

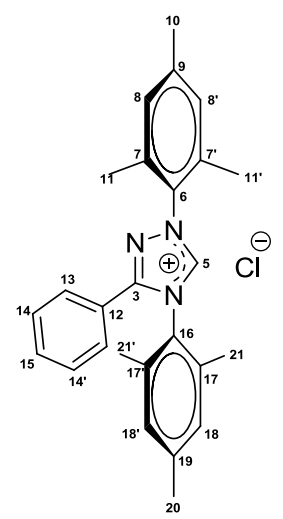

To the crude 20a (3.0 g, $8.07 \mathrm{mmol}, 1.0$ equiv) was added chlorobenzene $(2.0 \mathrm{~mL})$, triethyl orthoformate $(8.0 \mathrm{~mL}, 48.4 \mathrm{mmol}, 6.0$ equiv) and anhydrous $\mathrm{HCl}$ (4 $\mathrm{M}$ in dioxane, $2.0 \mathrm{~mL}, 8.07 \mathrm{mmol}, 1.0$ equiv). The flask was purged with argon and immersed in a silicon oil bath at 120 ${ }^{\circ} \mathrm{C}$. The mixture was stirred for $2 \mathrm{~h}$, resulting in a light orange homogeneous solution. The reaction mixture was allowed to cool to ambient temperature, and the solvent was removed on a rotary evaporator and a high vacuum, affording a light orange semi-solid. Purification by column chromatography, eluting with $\mathrm{MeOH} / \mathrm{EtOAc}$ (10:90), yielded the triazolium salt as light yellow brown solid $21 \mathrm{a} \cdot \mathrm{HCl}\left(3.0 \mathrm{~g}, 39 \%\right.$ yield): $\mathrm{mp} 212-214{ }^{\circ} \mathrm{C} ;{ }^{1} \mathbf{H}$ NMR (600 MHz, $\left.\mathbf{C D C l}_{3}\right): \delta[p p m]=13.18(\mathrm{~s}, 1 \mathrm{H}, \mathrm{H} 5), 7.57-7.54(\mathrm{~m}, 1 \mathrm{H}, \mathrm{H} 15), 7.48-7.46(\mathrm{~m}$, 2H, H14, H'14), 7.42-7.29 (m, 2H, H13, H13'), 7.06 (s, 2H, H8, H8'), 7.05 (s, 2H, H18, H18'), 2.36 (s, 3H, H10), 2.36 (s, 3H, H20), 2.29 (s, 6H, H11, H11'), 2.14 (s, 6H, H21, H21'); ${ }^{13} \mathrm{C}$ NMR (150 MHz, $\left.\mathbf{C D C l}_{3}\right): \delta[\mathrm{ppm}]=153.54$ (1C, C3), 148.50 (1C, C5), 142.26 (1C, C9), 142.01 (1C, C19), 134.46 (2C, C7, C7'), 134.25 (2C, C17, C17'), 132.84 (1C, C16), 131.19 (1C, C6), 130.61 (2C, C8, C8'), 129.91 (2C, C18, C18'), 129.55 (2C, C14, C14'), 127.79 (1C, C15), 127.68 (2C, C13, C13'), 122.55 (1C, C12), 21.30 (1C, C10), 21.25 (1C, C20), 18.04 (2C, C21, C21'), 17.76 (2C, C11, C11'); IR (ATR): $\tilde{v}\left[\mathrm{~cm}^{-1}\right]=2918,1604,1550,1521,1479$, 1446, 1379, 1330, 1224, 1037, 974, 945, 854, 779, 725, 692. HR-MS (ESI, positive): m/z calcd for $\left[\mathrm{C}_{26} \mathrm{H}_{28} \mathrm{~N}_{3}\right]^{+}[\mathrm{M}]^{+} 382.2278$ found 382.2277; Elemental Anal. Calcd for $\mathrm{C}_{26} \mathrm{H}_{28} \mathrm{CIN}_{3}$ : C, 74.71; H, 6.75; N, 10.05. Found: C, 74.39; H, 6.83; N, 9.98. 


\subsection{Synthesis of 1,4-bis[2,6-di(2-propyl)phenyl]-3-phenyl-1H-1,2,4-triazol-4-ium chloride $(21 \mathrm{~b} \cdot \mathrm{HCl})$}

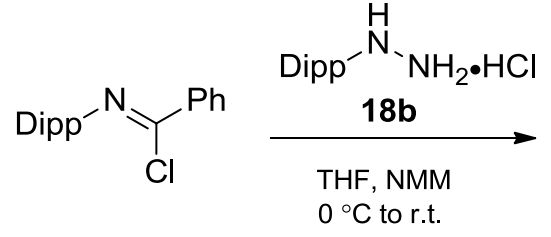

$16 b$

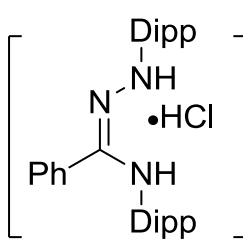

$20 b$

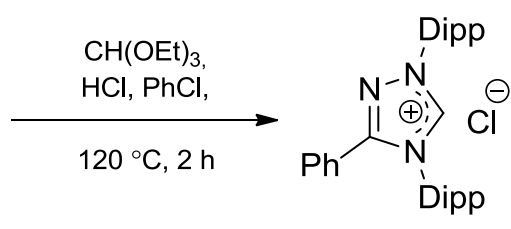

21b. $\mathrm{HCl}$

A flame-dried round-bottomed flask was charged with the imidoyl chloride $\mathbf{1 6 b}(5.24 \mathrm{~g}, 17.5$ mmol, 0.8 equiv), THF (150 ml) and Dipp-hydrazinium hydrochloride 18b $(5.0 \mathrm{~g}, 21.86 \mathrm{mmol}$, 1.0 equiv). The reaction mixture was cooled to $0^{\circ} \mathrm{C}$ and $\mathrm{N}$-methylmorpholine $(2.4 \mathrm{ml}, 21.86$ mmol, 1.0 equiv) was added. The reaction mixture was stirred at $0{ }^{\circ} \mathrm{C}$ for $3 \mathrm{~h}$. The ice-bath was removed and stirring was continued overnight. $\mathrm{MeOH}(15 \mathrm{ml})$ was added and the mixture was stirred for $30 \mathrm{~min}$. The solvent was removed under reduced pressure at $20^{\circ} \mathrm{C}$. Water $(100 \mathrm{ml})$ was added and the mixture was extracted with DCM $(500 \mathrm{ml})$ to afford the crude hydrazonamide hydrochloride $20 \mathrm{~b}(80 \%, 8.6 \mathrm{~g})$, which was used in the next step without further purification.

To the crude $20 \mathrm{~b}$ ( $8.6 \mathrm{~g}, 18.87 \mathrm{mmol}, 1.00$ equiv) was added chlorobenzene (6.0 mL), triethyl orthoformate (25.0 mL, $151 \mathrm{mmol}, 8.0$ equiv) and anhydrous $\mathrm{HCl}(4 \mathrm{M}$ in dioxane, $5.0 \mathrm{~mL}$,

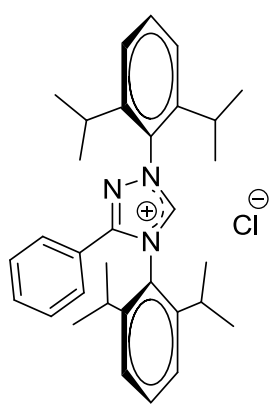

18.87 mmol, 1.00 equiv). The flask was purged with argon and immersed in a silicon oil bath at $120^{\circ} \mathrm{C}$. The mixture was stirred for $2 \mathrm{~h}$, resulting in a light orange homogeneous solution. The solution was allowed to cool to ambient temperature, and the solvent was removed on a rotary evaporator and a high vacuum. The resulting light orange semi solid was washed with EtOAc several times to give the triazolium salt $\mathbf{2 1} \mathbf{b} \cdot \mathbf{H C l}$ as white solid $(3.0$ g, 32\% yield): mp 264-265 ${ }^{\circ} \mathrm{C} ;{ }^{1} \mathrm{H}$ NMR (600 MHz, $\left.\mathbf{C D C l}_{3}\right): \delta[\mathrm{ppm}]=14.13$ $(\mathrm{s}, 1 \mathrm{H}), 7.62-7.60(\mathrm{~m}, 1 \mathrm{H}), 7.58-7.56(\mathrm{~m}, 1 \mathrm{H}), 7.53-7.50(\mathrm{~m}, 1 \mathrm{H}), 7.46-7.44(\mathrm{~m}, 2 \mathrm{H})$, 7.37$7.35(\mathrm{~m}, 6 \mathrm{H}), 2.53\left(\mathrm{sept}, 2 \mathrm{H},{ }^{3} \mathrm{~J}_{H H}=6.9 \mathrm{~Hz}\right), 2.41\left(\mathrm{sept}, 2 \mathrm{H},{ }^{3} \mathrm{~J}_{H H}=6.9 \mathrm{~Hz}\right), 1.36\left(\mathrm{~d}, 6 \mathrm{H},{ }^{3} \mathrm{~J}_{H H}\right.$ $=6.9 \mathrm{~Hz}), 1.35\left(\mathrm{~d}, 6 \mathrm{H},{ }^{3} J_{H H}=6.9 \mathrm{~Hz}\right), 1.27\left(\mathrm{~d}, 6 \mathrm{H},{ }^{3} J_{H H}=6.9 \mathrm{~Hz}\right), 1.01\left(\mathrm{~d}, 6 \mathrm{H},{ }^{3} J_{H H}=6.9 \mathrm{~Hz}\right)$; ${ }^{13} \mathrm{C}$ NMR (150 MHz, $\left.\mathrm{CDCl}_{3}\right): \delta[\mathrm{ppm}]=153.79,150.04,145.28,144.77,132.86,132.84$, 131.07, 129.41, 128.10, 127.56, 125.40, 124.52, 122.45, 29.68, 29.46, 24.79, 24.35, 23.58, 22.57; IR (ATR): $\tilde{v}\left[\mathrm{~cm}^{-1}\right]=2962,2870,2588,1548,1521,1460,1444,1367,1325$, 1263,1184, 1062, 937, 804, 758, 690; HR-MS (ESI, positive): $\mathrm{m} / z$ calcd for $\left[\mathrm{C}_{32} \mathrm{H}_{40} \mathrm{~N}_{3}\right]^{+}[\mathrm{M}]^{+}$ 466.3216 found 466.3214; Elemental Anal. Calcd for $\mathrm{C}_{32} \mathrm{H}_{40} \mathrm{CIN}_{3} .1 / 2$ EtOAc: $\mathrm{C}, 74.77 ; \mathrm{H}$, 8.12; N, 7.69. Found: C, 74.91; H, 8.02; N, 7.64. 


\section{Synthesis of bis-Ipp/BPh-substituted triazolium salts}

\subsection{Synthesis of 1,4-bis[2(2-propyl)phenyl]-3-phenyl-1,2,4-triazol-4-ium tetraphenylborate $\left(21 \mathrm{c} \bullet \mathrm{HBPh}_{4}\right)$}

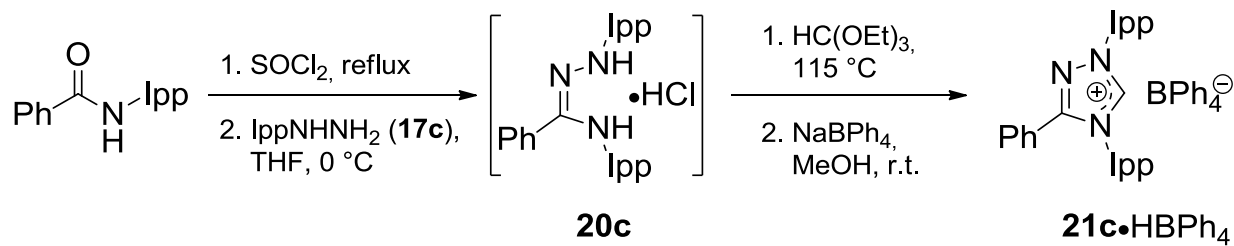

Thionyl chloride $\left(2.25 \mathrm{ml}, 31.0 \mathrm{mmol}, 5\right.$ equiv) was added to $N$-lpp-benzamide ${ }^{[13]}(1.479 \mathrm{~g}$, $6.2 \mathrm{mmol}, 1.0$ equiv) in a $50 \mathrm{ml}$ Schlenk flask flushed with a argon. The solution was heated to reflux for $2 \mathrm{~h}$. After cooling to r.t., the excess thionyl chloride was removed under reduced pressure. The residue was dissolved in absolute THF $(15 \mathrm{ml})$ and cooled to $0^{\circ} \mathrm{C}$. Ipp-hydrazine $17 \mathrm{c}^{[14]}$ (1.665 g, $11.1 \mathrm{mmol}, 1.8$ equiv) was dissolved in absolute THF (15 ml) in another Schlenk flask and cooled to $0^{\circ} \mathrm{C}$ under argon atmosphere. The reaction mixture was added to the solution of hydrazine 17c by means of a syringe, and the mixture was stirred for $2 \mathrm{~h}$ at $0{ }^{\circ} \mathrm{C}$. THF was evaporated under reduced pressure to give the crude hydrazonamide-hydrochloride 20c, which was used in the next step without further purification.

To this hydrazonamide hydrochloride $20 \mathrm{c}$ was added triethyl orthoformate $(5.16 \mathrm{ml}$, $31 \mathrm{mmol}, 5$ equiv), and the reaction mixture was heated to $115^{\circ} \mathrm{C}$ for $2 \mathrm{~h}$. The excess triethyl
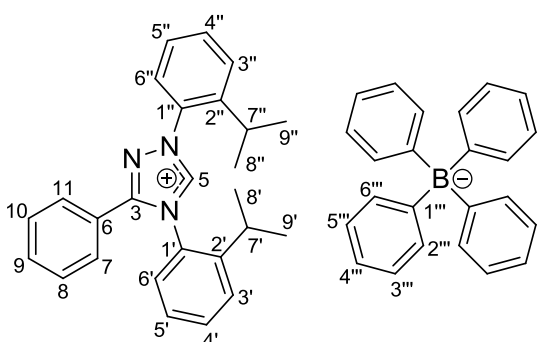
orthoformate was evaporated under reduced pressure. To the resulting residue was added $\mathrm{NaBPh}_{4}(2.122 \mathrm{~g}$, $6.2 \mathrm{mmol}, 1$ equiv) and $\mathrm{MeOH}(35 \mathrm{ml})$. From this solution, colorless crystals of the product $21 \mathrm{c} \cdot \mathrm{HBPh}_{4}$ slowly precipitated (1.4 g, 32\% yield). mp.: $188-189{ }^{\circ} \mathrm{C} ;{ }^{1} \mathrm{H}-\mathrm{NMR}$ $\left(600 \mathrm{MHz}, \mathrm{CDCl}_{3}\right): \delta[\mathrm{ppm}]=7.58(\mathrm{~m}, 1 \mathrm{H}, \mathrm{Har}), 7.53(\mathrm{~m}$, 1H, Har), 7.48-7.52 (m, 2H, Har), 7.33-7.36 (m, 5H, Har), 7.23-7.26 (m, 8H, Har), 7.10 (m, $2 \mathrm{H}, \mathrm{Har}$ ), 6.80-6.85 (m, 13H, Har), 5.94 (dd, $\left.{ }^{3} \mathrm{~J}=8.0 \mathrm{~Hz},{ }^{4} \mathrm{~J}=0.9 \mathrm{~Hz}, 1 \mathrm{H}, \mathrm{Har}\right), 5.73(\mathrm{~s}, 1 \mathrm{H}$, H5), 2.44 (sept, ${ }^{3} J=6.8 \mathrm{~Hz}, 1 \mathrm{H}, 7^{\prime \prime}$ ), 1.88 (sept, $\left.{ }^{3} \mathrm{~J}=6.8 \mathrm{~Hz}, 1 \mathrm{H}, \mathrm{H} 77^{\prime}\right), 1.24$ (d, ${ }^{3} \mathrm{~J}=6.8 \mathrm{~Hz}$, $\left.3 \mathrm{H}, \mathrm{H} 8 ", 9^{\prime \prime}\right), 1.20$ (d, $\left.{ }^{3} \mathrm{~J}=6.8 \mathrm{~Hz}, 3 \mathrm{H}, \mathrm{H} 8^{\prime \prime}, 9^{\prime \prime}\right), 1.05$ (d, $\left.{ }^{3} \mathrm{~J}=6.8 \mathrm{~Hz}, 3 \mathrm{H}, \mathrm{H} 8^{\prime}, 9^{\prime}\right), 0.67$ (d, ${ }^{3} \mathrm{~J}=$ $\left.6.8 \mathrm{~Hz}, 3 \mathrm{H}, \mathrm{H}^{\prime}, 9^{\prime}\right) ;{ }^{13} \mathrm{C}-\mathrm{NMR}\left(150 \mathrm{MHz}, \mathrm{CDCl}_{3}\right): \delta[\mathrm{ppm}]=163.7$ (q, $\left.{ }^{1} \mathrm{~J}=49.3 \mathrm{~Hz}, 4 \mathrm{C}, \mathrm{C} 1^{\prime \prime \prime}\right)$, 154.1 (1C, C3), 145.0 (1C, C5), 143.9 (1C, Cq), 143.5 (1C, Cq), 136.4 (q, ${ }^{3} \mathrm{~J}=1.6 \mathrm{~Hz}, 8 \mathrm{C}$, C3"',5"'), 133.1 (1C, CH), 132.7 (1C, CH), 132.7 (1C, CH), 132.0 (1C, Cq), $129.4(2 \mathrm{C}, \mathrm{CH})$, $128.6(1 \mathrm{C}, \mathrm{CH}), 128.5(2 \mathrm{C}, \mathrm{CH}), 128.5(1 \mathrm{C}, \mathrm{Cq}), 128.2(1 \mathrm{C}, \mathrm{CH}), 127.5(1 \mathrm{C}, \mathrm{CH}), 127.5(1 \mathrm{C}$, $\mathrm{CH}), 127.1$ (1C, CH), 126.8 (1C, CH), 125.6 (q, $\left.{ }^{2} \mathrm{~J}=3.0 \mathrm{~Hz}, 8 \mathrm{H}, \mathrm{H} 2^{\prime \prime \prime}, \mathrm{H} 66^{\prime \prime \prime}\right), 121.9$ (4H, H4"'), $121.6(1 \mathrm{C}, \mathrm{Cq}), 28.6$ (1C, C7"), 28.5 (1C, C7'), 24.1 (1C, C8',9'), 23.5 (1C, C8",9"), 23.4 (1C, 
C8",9"), 22.0 (1C, C8',9'); ${ }^{11}$ B-NMR (96 MHz, CDCl 3 ): $\delta[p p m]=6.81\left(\mathrm{BPh}_{4}\right)$; IR (ATR): $\tilde{v}$ $\left[\mathrm{cm}^{-1}\right]=3061(\mathrm{w}), 1555(\mathrm{~m}), 1479(\mathrm{~m}), 1597(\mathrm{~m}), 1449(\mathrm{~m}), 768(\mathrm{~m}), 729(\mathrm{~m}), 702(\mathrm{~s})$, $691(\mathrm{~m}), 610(\mathrm{~m})$; Elemental Anal. Calcd for $\mathrm{C}_{50} \mathrm{H}_{48} \mathrm{BN}_{3}$ : C, 85.58; $\mathrm{H}, 6.89$; N, 5.99; Found: C, 85.52; H, 6.88; N, 5.96 .

\subsection{Synthesis of 1,4-bis[(2-phenyl)phenyl]-3-phenyl-1,2,4-triazol-4-ium tetraphenylborate $\left(21 \mathrm{~d} \bullet \mathrm{HBPh}_{4}\right)$}

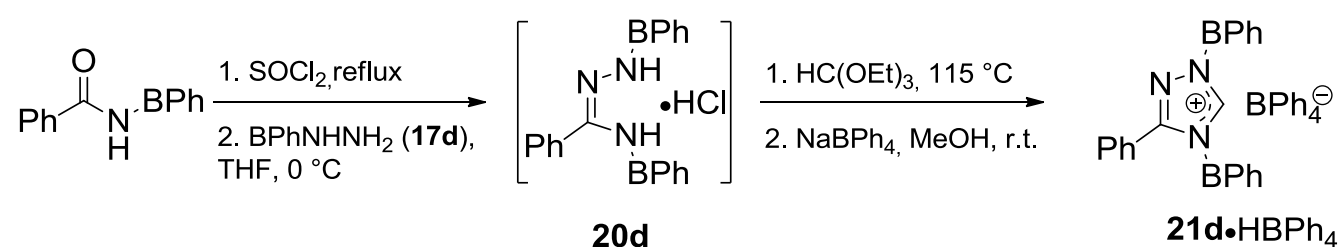

Thionyl chloride $\left(2.8 \mathrm{ml}, 38.25 \mathrm{mmol}, 5\right.$ equiv) was added to $\mathrm{N}$-BPh-benzamide ${ }^{15}(2.091 \mathrm{~g}$, $7.3 \mathrm{mmol}, 0.95$ equiv) in a $50 \mathrm{ml}$ Schlenk flask flushed with argon. The solution was heated to reflux for $1.5 \mathrm{~h}$. After cooling to rt, the excess thionyl chloride was removed under reduced pressure. The residue was dissolved in absolute THF $(20 \mathrm{ml})$ and cooled to $0{ }^{\circ} \mathrm{C}$. The o-biphenylhydrazine $17 \mathbf{d}^{[16]}$ (1.410 g, $7.65 \mathrm{mmol}, 1$ equiv) was dissolved in absolute THF $(20 \mathrm{ml})$ in another Schlenk flask and cooled to $0^{\circ} \mathrm{C}$ under argon atmosphere. The reaction mixture was added to the solution of hydrazine $17 \mathbf{d}$ means of a syringe, and the mixture was stirred for $2 \mathrm{~h}$ at $0^{\circ} \mathrm{C}$. THF was evaporated under reduced pressure to give the crude hydrazonamide-hydrochloride $\mathbf{2 0 d}$, which was used in the next step without further purification.

To this hydrazonamide hydrochloride $20 \mathrm{~d}$ was added triethyl orthoformate $(6.4 \mathrm{ml}$, $38.25 \mathrm{mmol}, 5$ equiv), and the reaction mixture was heated to $115^{\circ} \mathrm{C}$ for $3 \mathrm{~h}$. The excess triethyl orthoformate was evaporated under reduced pressure at r.t. and then at $80^{\circ} \mathrm{C}$. The residue was dissolved in $50 \mathrm{ml}$ of $\mathrm{MeOH}$ and $\mathrm{NaBPh}_{4}(2.618 \mathrm{~g}, 7.65 \mathrm{mmol}, 1$ equiv) was added. The mixture was stirred for $2 \mathrm{~h}$ at r.t., resulting the precipitation of a white powder.

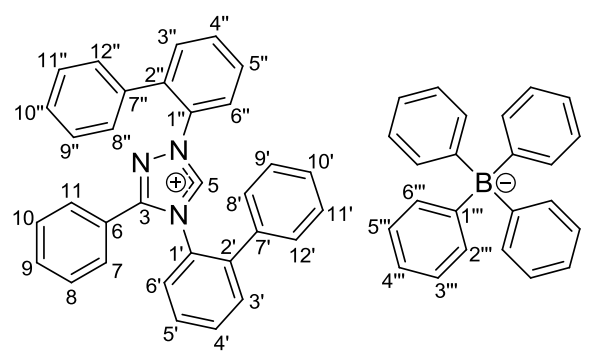

The solid was collected by filtration and recrystallized first from $\mathrm{MeOH} / \mathrm{DCM}$. A second recrystallization from $\mathrm{DCM} / n$ pentane afforded $\mathbf{2 1 d} \cdot \mathrm{HBPh}_{4}(772.0 \mathrm{mg}, 14 \%)$ as a faintly yellow solid containing DCM; mp.: $213-214{ }^{\circ} \mathrm{C}$; ${ }^{1} \mathrm{H}-\mathrm{NMR}$ (600 MHz, DMSO- $\left.d_{6}\right): \delta[p p m]=11.18(\mathrm{~s}, 1 \mathrm{H}, \mathrm{H} 5), 8.06$ $\left(\mathrm{dd},{ }^{3} \mathrm{~J}=7.6 \mathrm{~Hz},{ }^{4} \mathrm{~J}=1.6 \mathrm{~Hz}, 1 \mathrm{H}\right), 7.97\left(\mathrm{dd},{ }^{3} \mathrm{~J}=7.8 \mathrm{~Hz},{ }^{4} \mathrm{~J}\right.$ $=1.3 \mathrm{~Hz}, 1 \mathrm{H}), 7.76-7.83(\mathrm{~m}, 4 \mathrm{H}), 7.71\left(\mathrm{dd},{ }^{3} \mathrm{~J}=7.5 \mathrm{~Hz},{ }^{4} \mathrm{~J}=1.6 \mathrm{~Hz}, 1 \mathrm{H}\right), 7.48\left(\mathrm{dd},{ }^{3} \mathrm{~J}=7.1\right.$ $\left.\mathrm{Hz},{ }^{4} \mathrm{~J}=2.1 \mathrm{~Hz}, 1 \mathrm{H}\right), 7.41-7.45(\mathrm{~m}, 4 \mathrm{H}), 7.36-7.38(\mathrm{~m}, 2 \mathrm{H}), 7.33\left(\mathrm{t},{ }^{3} \mathrm{~J}=7.5 \mathrm{~Hz}, 1 \mathrm{H}\right), 7.16-$ $7.22(\mathrm{~m}, 12 \mathrm{H}), 6.93\left(\mathrm{~m}, 8 \mathrm{H}, \mathrm{H} 3^{\prime \prime \prime}, 5^{\prime \prime \prime}\right), 6.79\left(\mathrm{t},{ }^{3} \mathrm{~J}=7.2 \mathrm{~Hz}, 4 \mathrm{H}, \mathrm{H} 4^{\prime \prime \prime}\right), 6.67(\mathrm{~m}, 2 \mathrm{H}), 6.54(\mathrm{~m}$, $2 \mathrm{H}, \mathrm{H7}, 11) ;{ }^{13} \mathrm{C}-\mathrm{NMR}(150 \mathrm{MHz}$, DMSO-d $): \delta[\mathrm{ppm}]=163.3\left(\mathrm{q},{ }^{1} \mathrm{~J}\left({ }^{11} \mathrm{~B}\right)=49.7 \mathrm{~Hz}\right.$; sept, ${ }^{1} \mathrm{~J}$ 
$\left.\left.\left({ }^{10} \mathrm{~B}\right)=16.6 \mathrm{~Hz}\right], 4 \mathrm{C}, \mathrm{C} 1{ }^{\prime \prime \prime}\right), 152.3(1 \mathrm{C}, \mathrm{C} 3), 146.1(1 \mathrm{C}, \mathrm{C} 5), 137.8(1 \mathrm{C}, \mathrm{Cq}), 136.8(1 \mathrm{C}, \mathrm{Cq})$, $136.4(1 \mathrm{C}, \mathrm{Cq}), 135.5$ (q, $\left.{ }^{3} \mathrm{~J}=1.4 \mathrm{~Hz}, 8 \mathrm{C}, \mathrm{C} 3^{\prime \prime \prime}, 5^{\prime \prime \prime}\right), 135.3(1 \mathrm{C}, \mathrm{Cq}), 132.3(1 \mathrm{C}, \mathrm{CH}), 132.2$ $(1 \mathrm{C}, \mathrm{Cq}), 132.1(1 \mathrm{C}, \mathrm{CH}), 131.9(1 \mathrm{C}, \mathrm{CH}), 131.8(1 \mathrm{C}, \mathrm{CH}), 131.4(1 \mathrm{C}, \mathrm{CH}), 129.5(1 \mathrm{C}, \mathrm{CH})$, $129.1(1 \mathrm{C}, \mathrm{CH}), 128.8(\mathrm{~s}, 1 \mathrm{C}, \mathrm{Cq}), 128.7(2 \mathrm{C}, \mathrm{CH}), 128.7(2 \mathrm{C}, \mathrm{CH}), 128.7(2 \mathrm{C}, \mathrm{CH}), 128.6$ $(2 \mathrm{C}, \mathrm{CH}), 128.2(1 \mathrm{C}, \mathrm{CH}), 128.0(1 \mathrm{C}, \mathrm{CH}), 127.9(2 \mathrm{C}, \mathrm{CH}), 127.9(1 \mathrm{C}, \mathrm{CH}), 127.5(2 \mathrm{C}, \mathrm{CH})$, $126.4(1 \mathrm{C}, \mathrm{CH}), 125.2$ (q, $\left.{ }^{2} \mathrm{~J}=2.8 \mathrm{~Hz}, 8 \mathrm{C}, \mathrm{C} 2{ }^{\prime \prime \prime}, 66^{\prime \prime \prime}\right), 121.6(1 \mathrm{C}, \mathrm{Cq}), 121.4\left(4 \mathrm{C}, \mathrm{C} 4^{\prime \prime \prime}\right) ;{ }^{11} \mathrm{~B}-$ NMR (160 MHz, DMSO-d $\left.d_{6}\right): \delta[p p m]=6.62\left(B P h_{4}\right)$; IR (ATR): $\tilde{v}\left[\mathrm{~cm}^{-1}\right]=3053(\mathrm{w}), 3036(\mathrm{w})$, $2984(w), 2361(w), 2332(w), 1578(w), 1551(w), 1477(m), 1447(w), 1425(w), 1265(w)$, $1206(w), 1182(w), 1121(w), 1067(w), 1032(w), 849(w), 760(m), 743(w), 733(w)$, $698(\mathrm{~s}), 691(\mathrm{~s}), 664(\mathrm{~m}), 610(\mathrm{~m})$; HR-MS: m/z [21d+H] $]^{+}$Calcd: 450.1964743; Found: 450.19610 .

\section{Synthesis of 2-[2,6-di(2-propyl)phenyl]-6,7-dihydro-5H-pyrrolo[2,1-c][1,2,4]triazol- 2-ium chloride (27)}

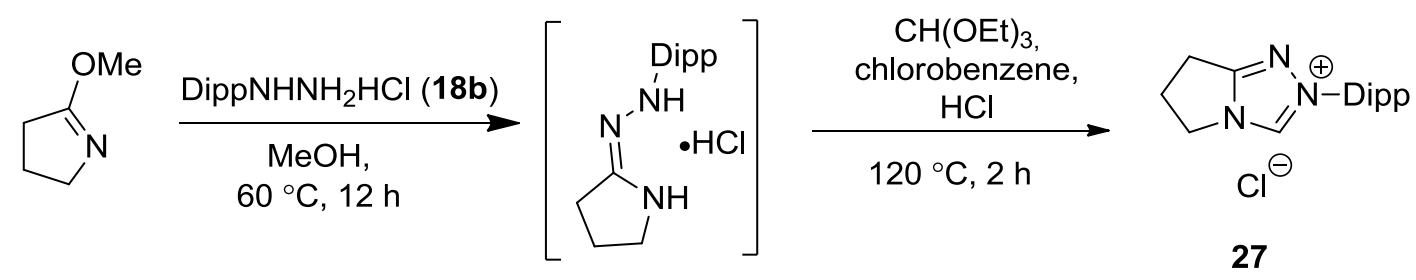

The synthesis of $\mathbf{2 7}$ was in analogy to the literature procedure reported by Bode et al. ${ }^{[17]}$

A flame-dried round-bottomed flask was charged with the Dipp-hydrazinium chloride $\mathbf{1 8 b}$ $(2.3 \mathrm{~g}, 10.1 \mathrm{mmol}, 1.0$ equiv) and $\mathrm{MeOH}(8 \mathrm{~mL})$. To this solution the imino ether $(1.0 \mathrm{~g}$, $10.1 \mathrm{mmol}, 1.0$ equiv) was added in a single portion and the mixture was stirred at ambient temperature (ca. $10 \mathrm{~min}$ ). A catalytic amount of anhydrous $\mathrm{HCl}(4 \mathrm{M}$ in dioxane, $0.03 \mathrm{~mL}$, $0.01 \mathrm{mmol}$ ) was added to the solution. The mixture was then heated to $60^{\circ} \mathrm{C}$ under an atmosphere of argon for $12 \mathrm{~h}$. The mixture was concentrated in vacuo to afford the crude hydrazonamide hydrochloride $(2.0 \mathrm{~g}, 70 \%$ yield), which was used in the next step without further purification.

To this hydrazonamide hydrochloride $(2.0 \mathrm{~g}, 6.76 \mathrm{mmol}, 1.0$ equiv) was added chlorobenzene $(6 \mathrm{~mL})$, triethyl orthoformate $(9.0 \mathrm{~mL}, 54.1 \mathrm{mmol}, 8.0$ equiv) and anhydrous

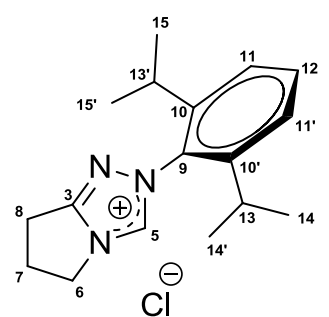
$\mathrm{HCl}$ (4 $\mathrm{M}$ in dioxane, $1.7 \mathrm{~mL}, 6.76 \mathrm{mmol}, 1.0$ equiv). The mixture was then heated at $120^{\circ} \mathrm{C}$ for $2 \mathrm{~h}$ and concentrated in vacuo. The resulting light brown solid was washed with EtOAc several times to give 27 (1.34 g, $4.39 \mathrm{mmol}, 65 \%$ yield) as light brown solid; ${ }^{1} \mathbf{H}$ NMR (600 MHz, DMSO- $\left.\boldsymbol{d}_{6}\right): \delta[\mathrm{ppm}]=10.6(\mathrm{~s}, 1 \mathrm{H}, \mathrm{H} 5), 7.67-7.64(\mathrm{~m}, 1 \mathrm{H}, \mathrm{H} 12), 7.47-$ $7.46\left(\mathrm{~m}, 2 \mathrm{H}, \mathrm{H} 11, \mathrm{H} 11^{\prime}\right), 4.50\left(\mathrm{t}, 2 \mathrm{H}, \mathrm{H} 6,{ }^{3} \mathrm{~J}_{H H}=7.38 \mathrm{~Hz}\right), 3.21(\mathrm{t}, 2 \mathrm{H}$, 
$\mathrm{H} 8,{ }^{3} \mathrm{~J}_{H H}=7.68 \mathrm{~Hz}$ ), 2.78 (quin, $2 \mathrm{H}, \mathrm{H} 7,{ }^{3} \mathrm{~J}_{H H}=7.5 \mathrm{~Hz}$ ), 2.45 (sept, $2 \mathrm{H}, \mathrm{H} 13, \mathrm{H} 13^{،},{ }^{3} \mathrm{~J}_{H H}=6.78$ $\mathrm{Hz}), 1.16\left(\mathrm{~d}, 6 \mathrm{H},{ }^{3} \mathrm{~J}_{\mathrm{HH}}=6.78 \mathrm{~Hz}\right), 1.10\left(\mathrm{~d}, 6 \mathrm{H},{ }^{3} \mathrm{~J}_{H H}=6.78 \mathrm{~Hz}\right) ;{ }^{13} \mathrm{C}$ NMR (150 MHz, DMSO$\left.\boldsymbol{d}_{6}\right): \delta[\mathrm{ppm}]=163.7(1 \mathrm{C}, \mathrm{C} 3), 146.3\left(2 \mathrm{C}, \mathrm{C} 10, \mathrm{C} 10^{\prime}\right), 132.7(1 \mathrm{C}, \mathrm{C} 12), 131.9(1 \mathrm{C}, \mathrm{C} 9), 124.8$ (2C, C11, C11 $\left.{ }^{\circ}\right), 47.8$ (1C, C6), 28.1 (2C, C13, C13'), 26.8, (1C, C7), 24.4 (2C, C14, C14'), 24.2 (2C, C15, C15'), 22.0 (1C, C8); HR-MS (ESI, positive): $\mathrm{m} / z$ calcd for $\left[\mathrm{C}_{17} \mathrm{H}_{24} \mathrm{~N}_{3}\right]^{+}[\mathrm{M}]^{+}$ 270.19647 found 270.19628 .

\section{Generation of the free carbenes $15 a-b$ and $21 a-d$}

15a: 2,5-Diphenyl-4-(2,4,6-trimethylphenyl)-2,4-dihydro-3H-1,2,4-triazol-3-ylidene<smiles></smiles>

$15 \mathrm{a} \cdot \mathrm{HCl}$

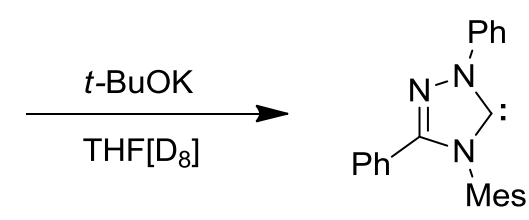

$15 \mathrm{a}$

In a glovebox, THF- $d_{8}(0.6 \mathrm{ml})$ was added to a mixture of the triazolium salt $15 \mathrm{a} \cdot \mathrm{HCl}(20 \mathrm{mg}$, $0.05 \mathrm{mmol}, 1$ equiv) and $t$-BuOK $(6.0 \mathrm{mg}, 0.05 \mathrm{mmol}, 1.0$ equiv) in a $5 \mathrm{ml}$ vial. The suspension was stirred for $5 \mathrm{~min}$. The resulting turbid solution was filtered via a syringe filter and transferred into a NMR tube. NMR spectroscopic analysis showed the signals of free carbene 17a; ${ }^{1} \mathbf{H}$ NMR (600 MHz, THF- $\left.\boldsymbol{d}_{8}\right): \delta[\mathrm{ppm}]=8.33\left(\mathrm{~d}, 2 \mathrm{H},{ }^{3} J_{H H}=8.1 \mathrm{~Hz}\right), 7.51-7.47$ $(\mathrm{m}, 4 \mathrm{H}), 7.41-7.39(\mathrm{~m}, 1 \mathrm{H}), 7.34-7.31(\mathrm{~m}, 3 \mathrm{H}), 7.05(\mathrm{~s}, 2 \mathrm{H}) 2.38(\mathrm{~s}, 3 \mathrm{H}), 2.0(\mathrm{~s}, 6 \mathrm{H}) ;{ }^{13} \mathrm{C}$ NMR $\left(150 \mathrm{MHz}\right.$, THF- $\left.\boldsymbol{d}_{8}\right): \delta[\mathrm{ppm}]=216.4,153.5,143.5,139.9,137.0,136.4,131.4,130.6,130.2$, $129.9,129.0,128.3,127.8,120.8,21.7,18.9$.

15b: 4-[2,6-Di(propan-2-yl)phenyl]-2,5-diphenyl-2,4-dihydro-3H-1,2,4-triazol-3-ylidene<smiles></smiles>

$15 \mathrm{~b} \cdot \mathrm{HCl}$<smiles>CC(C)(C)OC(C)(C)C</smiles>

${ }_{\text {Dipp }}$

$15 b$

In a glovebox, THF- $d_{8}(0.6 \mathrm{ml})$ was added to a mixture of the triazolium salt $\mathbf{1 5 b} \cdot \mathrm{HCl}(20 \mathrm{mg}$, $0.048 \mathrm{mmol}, 1$ equiv) and $t$-BuOK $(5.3 \mathrm{mg}, 0.048 \mathrm{mmol}, 1.0$ equiv) in a $5 \mathrm{ml}$ vial. The suspension was stirred for $5 \mathrm{~min}$. The resulting turbid solution was filtered via a syringe filter and transferred into a NMR tube. NMR spectroscopic analysis showed the signals of free carbene 17b; ${ }^{1} \mathbf{H}$ NMR $\left(600 \mathrm{MHz}\right.$, THF- $\left.\boldsymbol{d}_{8}\right): \delta[\mathrm{ppm}]=8.36-8.34(\mathrm{~m}, 2 \mathrm{H}), 7.52-7.48(\mathrm{~m}, 5 \mathrm{H})$, 
7.40-7.37 (m, 1H), 7.35-7.30 (m, 5H), 2.64 (sept, $\left.2 \mathrm{H},{ }^{3} J_{H H}=6.8 \mathrm{~Hz}\right), 1.17\left(\mathrm{~d}, 6 \mathrm{H},{ }^{3} J_{H H}=6.8\right.$ $\mathrm{Hz}), 0.96\left(\mathrm{~d}, 6 \mathrm{H},{ }^{3} \mathrm{~J}_{H H}=6.8 \mathrm{~Hz}\right) ;{ }^{13} \mathrm{C}$ NMR $\left(150 \mathrm{MHz}\right.$, THF- $\left.d_{8}\right): \delta[\mathrm{ppm}]=216.7,154.0,147.0$, 143.5, 136.7, 131.0, 130.2, 129.8, 129.5, 128.1, 125.4, 120.9, 30.0, 25.4, 23.2.

21a: 5-Phenyl-2,4-bis(2,4,6-trimethylphenyl)-2,4-dihydro-3H-1,2,4-triazol-3-ylidene<smiles>CN1CN(C)C(c2ccccc2)=N1</smiles>

In a glove box, a $25 \mathrm{ml}$ round-bottomed flask was charged with the triazolium salt $21 \mathrm{a} \cdot \mathrm{HCl}$ (300 mg, $0.72 \mathrm{mmol}, 1.0$ equiv), $\mathrm{KH}$ (28.8 mg, $0.72 \mathrm{mmol}, 1.0$ equiv), $t$-BuOK (8 mg, 0.07 mmol, 0.1 equiv) and THF (5 mL). The reaction mixture was stirred at room temperature for $1 \mathrm{~h}$. The resulting $\mathrm{KCl}$ precipitation was removed by filtration and the filtrate was evaporated to give a white solid. Recrystallization from a mixture of pentane and THF (3:1) furnished 21a (239 mg, 78\% yield) as white crystalline solid; mp.: $164-165{ }^{\circ} \mathrm{C} ;{ }^{1} \mathbf{H}$ NMR (600 MHz, THF- $\boldsymbol{d}_{8}$ ): $\delta$ [ppm] = 7.46-7.45 (m, 2H, H13, H13'), 7.37-7.35 (m, 1H, H15), 7.30-7.27 (12) (m, 2H, H14, H14'), 7.05 (s, 2H, H18, H18'), 7.04 (s, 2H, H8, H8'), 2.38 (s, $6 \mathrm{H}, \mathrm{H} 10, \mathrm{H} 20), 2.20$ (s, 6H, H11, H11'), 2.05 (s, 6H, H21, H21'); ${ }^{13} \mathrm{C}$ NMR $\left(150 \mathrm{MHz}\right.$, THF- $\left.d_{8}\right): \delta[\mathrm{ppm}]=218.05(1 \mathrm{C}, \mathrm{C} 5), 152.5(1 \mathrm{C}, \mathrm{C} 3), 139.5(1 \mathrm{C}$, C6), 139.2 (1C, C19), 138.7 (1C, C9), 136.7 (1C, C16), 136.1 (2C, C17, C17'), 136.02 (2C, C7, C7'), 130.6 (1C, C15), 130.1 (2C, C18, C18'), 129.5 (2C, C8, C8'), 129.4 (2C, C14, C14'), 128.4 (2C, CC13, C13'), 128.2 (1C, C12), 21.28 (1C, C20), 21.27 (1C, C10), 18.46 (2C, C21, C21'), 18.26 (2C, C11, C11').

21b: 2,4-Bis[2,6-di(propan-2-yl)phenyl]-5-phenyl-2,4-dihydro-3H-1,2,4-triazol-3-ylidene<smiles></smiles>

21b. $\mathrm{HCl}$

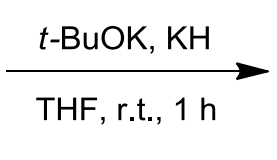

Ph

21b

In a glove box, a $25 \mathrm{ml}$ round-bottomed flask was charged with the triazolium salt $\mathbf{2 1} \mathbf{b} \cdot \mathbf{H C l}$ (300 mg, 0.597 mmol, 1.0 equiv), KH (23.96 mg, 0.597 mmol, 1.0 equiv), $t$-BuOK (6.7 mg, 
$0.06 \mathrm{mmol}, 0.1$ equiv) and THF ( $5 \mathrm{~mL})$. The reaction mixture was stirred at room temperature for $1 \mathrm{~h}$. The resulting $\mathrm{KCl}$ precipitation was removed by filtration and the filtrate was evaporated to give a white solid. Recrystallization from a mixture of pentane and THF (3:1) furnished 21b (223 mg, 80\% yield) as white crystalline solid; mp.: 167-168 ${ }^{\circ} \mathrm{C}$; ${ }^{1} \mathbf{H}$ NMR (600

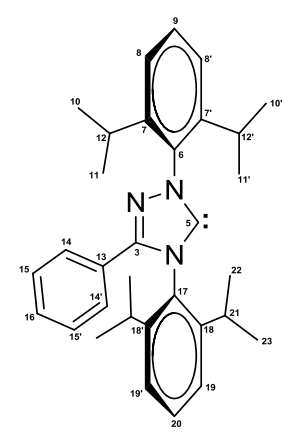

MHz, THF- $\left.\boldsymbol{d}_{8}\right): \delta[\mathrm{ppm}]=7.51-7.48(\mathrm{~m}, 1 \mathrm{H}, \mathrm{H} 20), 7.47-7.45(\mathrm{~m}, 3 \mathrm{H}, \mathrm{H} 9$, $\mathrm{H} 14, \mathrm{H} 14$ '), 7.37-7.34 (m, 5H, H16, H8, H8', H19, H19'), 7.30-7.27 (m, 2H, H15, H15'), 2.94 (sept, $2 \mathrm{H},{ }^{3} J_{H H}=6.9 \mathrm{~Hz}, \mathrm{H} 12, \mathrm{H} 12$ '), 2.74 (sept, $2 \mathrm{H},{ }^{3} J_{H H}$ $\left.=6.9 \mathrm{~Hz}, \mathrm{H} 21, \mathrm{H} 21^{\prime}\right), 1.27$ (d, 6H, ${ }^{3} \mathrm{~J}_{\mathrm{HH}}=6.9 \mathrm{~Hz}, \mathrm{H} 10, \mathrm{H} 10$ '), 1.24 (d, 6H, $\left.{ }^{3} J_{H H}=6.9 \mathrm{~Hz}, \mathrm{H} 11, \mathrm{H} 11^{\prime}\right), 1.21\left(\mathrm{~d}, 6 \mathrm{H},{ }^{3} J_{H H}=6.9 \mathrm{~Hz}, \mathrm{H} 23, \mathrm{H} 23\right.$ '), 1.03 (d, $6 \mathrm{H},{ }^{3} \mathrm{~J}_{H H}=6.9 \mathrm{~Hz}, \mathrm{H} 22, \mathrm{H} 22$ '); ${ }^{13} \mathbf{C}$ NMR (150 MHz, THF- $\left.d_{8}\right): \delta[\mathrm{ppm}]=$ 219.0 (1C, C5), 152.8 (1C, C3), 147.0 (2C, C7, C7'), 146.6 (2C, C18, C18'), 139.5 (1C, C6), 136.5 (1C, C17), 130.8 (1C, C16), 130.5 (1C, C20), 130.2 (1C, C9), 129.4 (2C, C15, C15'), 129.1 (2C, C14, C14'), 128.0 (1C, C13), 124.9 (2C, C19, C19'), 124.2 (2C, C8, C8'), 29.9 (2C, C12, C12'), 29.7 (2C, C21, C21'), 25.0 (2C, C23, C23'), 24.6 (2C, C11, C11'), 23.9 (2C, C10, C10'), 22.7 (2C, C22, C22').

\section{1c: 2,4-Bis[2(propan-2-yl)phenyl]-5-phenyl-2,4-dihydro-1,2,4-triazol-3-ylidene}

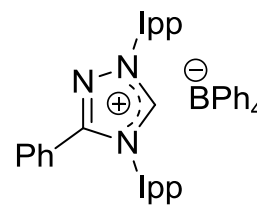

21c. $\cdot \mathrm{HBPh}_{4}$<smiles></smiles>

21c

In a glovebox, a mixture of $\mathbf{2 1} \mathrm{c} \cdot \mathrm{HBPh}_{4}(42,0 \mathrm{mg}, 0,06 \mathrm{mmol})$, potassium hydride $(5,4 \mathrm{mg}$, $0,135 \mathrm{mmol}), t$-BuOK $(0,1 \mathrm{mg}, 0,891 \mu \mathrm{mol})$ and THF- $d_{8}(0.4 \mathrm{ml})$ was stirred for $3 \mathrm{~h}$. The resulting suspension was passed through a syringe filter. The precipitate was additionally washed with THF- $d_{8}$. The combined filtrate was transferred to a NMR tube, which was then sealed with a septum. The NMR analysis showed the signals of free carbene $21 \mathrm{c} ;{ }^{1} \mathbf{H}-\mathbf{N M R}$ $\left(600 \mathrm{MHz}, \mathrm{THF}-\boldsymbol{d}_{8}\right): \delta[\mathrm{ppm}]=7.54\left(\mathrm{dd},{ }^{3} \mathrm{~J}=7.8 \mathrm{~Hz},{ }^{4} \mathrm{~J}=1.6 \mathrm{~Hz}, 1 \mathrm{H}, \mathrm{Har}\right), 7.50-7.38(\mathrm{~m}, 6 \mathrm{H}$, Har), 7.34-7.25 (m, 6H, Har), 3.56 (sept, ${ }^{3} \mathrm{~J}=6.9 \mathrm{~Hz}, 1 \mathrm{H}, \mathrm{CH}\left(\mathrm{CH}_{3}\right)_{2}$ ), 2.84 (sept, ${ }^{3} \mathrm{~J}=6.9 \mathrm{~Hz}$, $\left.1 \mathrm{H}, \mathrm{CH}\left(\mathrm{CH}_{3}\right)_{2}\right), 1.25-1.23\left(\mathrm{~m}, 6 \mathrm{H}, \mathrm{CH}_{3}\right), 1.17-1.15\left(\mathrm{~m}, 3 \mathrm{H}, \mathrm{CH}_{3}\right), 0.94-0.92\left(\mathrm{~m}, 3 \mathrm{H}, \mathrm{CH}_{3}\right)$; ${ }^{13} \mathrm{C}-N M R\left(151 \mathrm{MHz}\right.$, THF- $\left.\boldsymbol{d}_{8}\right): \delta[\mathrm{ppm}]=217.9(1 \mathrm{C}, \mathrm{Cq}), 152.8(1 \mathrm{C}, \mathrm{Cq}), 146.2(1 \mathrm{C}, \mathrm{Cq})$, $145.0(1 \mathrm{C}, \mathrm{Cq}), 141.2(1 \mathrm{C}, \mathrm{Cq}), 138.5(1 \mathrm{C}, \mathrm{Cq}), 130.7$ (1C, CH), $130.2(1 \mathrm{C}, \mathrm{CH}), 129.5(2 \mathrm{C}$, $\mathrm{CH}), 129.4$ (1C, CH), 129.3 (2C, CH), 129.2 (1C, CH), 128.1 (1C, CH), $127.8(1 \mathrm{C}, \mathrm{Cq}), 127.6$ $(1 \mathrm{C}, \mathrm{CH}), 127.5(1 \mathrm{C}, \mathrm{CH}), 127.2(1 \mathrm{C}, \mathrm{CH}), 126.9(1 \mathrm{C}, \mathrm{CH}), 29.2\left(1 \mathrm{C}, \mathrm{CH}\left(\mathrm{CH}_{3}\right)_{2}\right), 29.0(1 \mathrm{C}$, $\left.\mathrm{CH}\left(\mathrm{CH}_{3}\right)_{2}\right), 24.8\left(1 \mathrm{C}, \mathrm{CH}_{3}\right), 24.3\left(1 \mathrm{C}, \mathrm{CH}_{3}\right), 24.1\left(1 \mathrm{C}, \mathrm{CH}_{3}\right), 22.6\left(1 \mathrm{C}, \mathrm{CH}_{3}\right)$. 
21d: 2,4-Bis[2(phenyl)phenyl]-5-phenyl-2,4-dihydro-1,2,4-triazol-3-ylidene

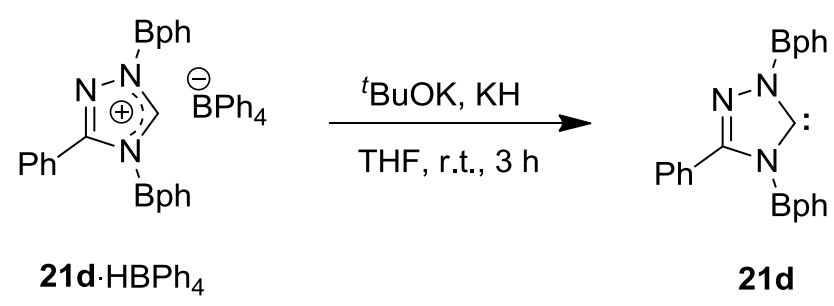

In a glovebox a mixture of $\mathbf{2 1 d} \cdot \mathrm{HBPh}_{4}(46,1 \mathrm{mg}, 0,060 \mathrm{mmol})$, potassium hydride $(3,6 \mathrm{mg}$, $0,090 \mathrm{mmol}), t$-BuOK $(0,1 \mathrm{mg}, 0,891 \mu \mathrm{mol})$ and THF $(0.4 \mathrm{ml})$ was stirred for $3 \mathrm{~h}$. The solution was concentrated under reduced pressure to remove residual DCM (incorporated in $\left.\mathbf{2 1 d} \cdot \mathrm{HBPh}_{4}\right) \cdot \mathrm{d}_{8}$-THF $(0.4 \mathrm{ml})$ was added and the resulting suspension was passed through a syringe filter. The filtrate was transferred to a NMR tube, which was sealed with a septum. The NMR analysis showed the signals of free carbene $21 \mathrm{~d}$; ${ }^{1} \mathrm{H}-\mathrm{NMR}$ (600 MHz, THF-d8): $\delta$ [ppm] = 7.75-7.72 (m, 1H), 7.59-7.55 (m, 1H), 7.50-7.43 (m, 5H), 7.32-7.29 (m, 2H), 7.28$7.24(\mathrm{~m}, 2 \mathrm{H}), 7.23-7.20(\mathrm{~m}, 1 \mathrm{H}), 7.17-1.12(\mathrm{~m}, 2 \mathrm{H}), 7.06-7.02(\mathrm{~m}, 2 \mathrm{H}), 6.99-6.96(\mathrm{~m}, 2 \mathrm{H})$, 6.70-6.67 (m, 2H), 6.64-6.61 (m, 2H); ${ }^{13} \mathrm{C}-\mathrm{NMR}$ (151 MHz, THF-d8): $\delta[p p m]=218.2(1 \mathrm{C}$, $\mathrm{Cq}), 152.6(1 \mathrm{C}, \mathrm{Cq}), 141.3(1 \mathrm{C}, \mathrm{Cq}), 141.1(1 \mathrm{C}, \mathrm{Cq}), 140.0(1 \mathrm{C}, \mathrm{Cq}), 139.4(1 \mathrm{C}, \mathrm{Cq}), 139.1$ $(1 \mathrm{C}, \mathrm{Cq}), 138.3(1 \mathrm{C}, \mathrm{Cq}), 132.1(1 \mathrm{C}, \mathrm{CH}), 131.6(1 \mathrm{C}, \mathrm{CH}), 130.2(2 \mathrm{C}, \mathrm{CH}), 130.1(1 \mathrm{C}, \mathrm{CH})$, 129.9 (3C, CH), $129.8(1 \mathrm{C}, \mathrm{CH}), 129.3(1 \mathrm{C}, \mathrm{CH}), 129.0(2 \mathrm{C}, \mathrm{CH}), 129.0(2 \mathrm{C}, \mathrm{CH}), 128.9(1 \mathrm{C}$, $\mathrm{CH}), 128.7(2 \mathrm{C}, \mathrm{CH}), 128.7(2 \mathrm{C}, \mathrm{CH}), 128.6(1 \mathrm{C}, \mathrm{CH}), 128.4(1 \mathrm{C}, \mathrm{CH}), 127.9(1 \mathrm{C}, \mathrm{Cq}), 127.7$ $(1 \mathrm{C}, \mathrm{CH}), 127.6(1 \mathrm{C}, \mathrm{CH})$.

\section{Synthesis of $\alpha, \beta$-unsaturated aldehydes}

The following enals were synthesized according to ref. ${ }^{[18]}$<smiles>C/C(C=O)=C\C1CCCCC1</smiles>

\section{(2E)-3-Cyclohexyl-2-methylprop-2-enal (25a)}

${ }^{1} \mathrm{H}$ NMR (300 MHz, $\left.\mathbf{C D C l}_{3}\right): \delta[p p m]=9.37(\mathrm{~s}, 1 \mathrm{H}), 6.32-6.28(\mathrm{~m}, 1 \mathrm{H}), 2.59-2.47(\mathrm{~m}, 1 \mathrm{H})$ 1.80-1.68 (m, 7H), 1.37-1.05 (m, 6H). $\left.{ }^{13} \mathbf{C ~ N M R ~ ( 7 5 ~ M H z , ~} \mathbf{C D C l}_{3}\right): \delta[p p m]=195.6,159.5$, 137.4, 37.9, 31.7, 25.8, 25.40, 9.17, IR (ATR): $\tilde{v}\left[\mathrm{~cm}^{-1}\right]=2924,2852,1685,1637,1448$, 1012, 671. HR-MS (EI) $\mathrm{m} / \mathrm{z}$ calcd for $\left[\mathrm{C}_{10} \mathrm{H}_{16} \mathrm{O}\right.$ ] [M] 152.1201 found 152.119. 
<smiles>CC/C(C=O)=C\c1ccccc1</smiles>

(2E)-2-[(4-Phenyl)methylidene]butanal (25g)

${ }^{1} \mathrm{H}$ NMR $\left(600 \mathrm{MHz}, \mathbf{C D C l}_{3}\right): \delta[\mathrm{ppm}]=9.55(\mathrm{~s}, 1 \mathrm{H}), 7.53-7.32(\mathrm{~m}, 5 \mathrm{H}), 7.20(\mathrm{~s}, 1 \mathrm{H}), 2.57(\mathrm{q}$, $\left.2 \mathrm{H},{ }^{3} \mathrm{~J}_{\mathrm{HH}}=7.5 \mathrm{~Hz}\right), 1.14\left(\mathrm{t}, 3 \mathrm{H},{ }^{3} \mathrm{~J}_{\mathrm{HH}}=7.5 \mathrm{~Hz}\right){ }^{13} \mathrm{C}$ NMR (150 MHz, $\left.\mathbf{C D C l}_{3}\right): \delta[\mathrm{ppm}]=195.5$, 149.5, 144.50, 134.94, 129.6, 129.5, 128.8, 18.05, 12.8. IR (ATR): $\tilde{v}\left[\mathrm{~cm}^{-1}\right]=2964,1680$, $1625,1448,1180,1056,1028,979,923,810,752,696$. HR-MS (EI) $m / z$ calcd for $\left[\mathrm{C}_{11} \mathrm{H}_{12} \mathrm{O}\right.$ ] 160.0888 ; found 160.088 .

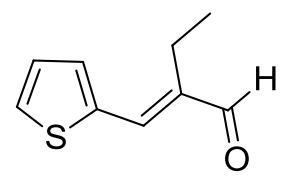

(2E)-2-[(Thiophen-2-yl)methylidene]butanal (25i)

${ }^{1} \mathrm{H}$ NMR (600 MHz, $\left.\mathbf{C D C l}_{3}\right): \delta[\mathrm{ppm}]=9.51(\mathrm{~s}, 1 \mathrm{H}), 7.61-7.60(\mathrm{~m}, 1 \mathrm{H}), 7.40-7.37(\mathrm{~m}, 1 \mathrm{H})$, $7.35(\mathrm{~s}, 1 \mathrm{H}), 7.18-7.17(\mathrm{~m}, 1 \mathrm{H}), 2.67\left(\mathrm{q}, 2 \mathrm{H},{ }^{3} \mathrm{~J}_{\mathrm{HH}}=7.5 \mathrm{~Hz}\right), 1.11(\mathrm{t}, 3 \mathrm{H}) .{ }^{13} \mathrm{C}$ NMR (150 MHz, $\left.\mathbf{C D C l}_{3}\right): \delta[\mathrm{ppm}]=194.4,141.4,141.2,138.03,133.05,130.9,127.8,18.40,12.09$. IR (ATR): $\tilde{v}\left[\mathrm{~cm}^{-1}\right]=2968,2933,1670,1616,1462,1417,1359,1246,1161,1051,977,856,796,702$. HR-MS (EI) $m / z$ calcd for [ $\mathrm{C}_{9} \mathrm{H}_{10} \mathrm{OS}$ ] [M] 166.0452 found 166.045 .<smiles>C/C(C=O)=C\c1ccco1</smiles>

(2E)-2-[(Furan-2-yl)methylidene]propanal (25c)

${ }^{1} \mathrm{H}$ NMR $\left(300 \mathrm{MHz}, \mathbf{C D C l}_{3}\right): \delta[\mathrm{ppm}]=9.51(\mathrm{~s}, 1 \mathrm{H}), 7.63(\mathrm{~s}, 1 \mathrm{H}), 7.04(\mathrm{~s}, 1 \mathrm{H}), 6.79-6.78(\mathrm{~m}$, $1 \mathrm{H}), 6.58-6.57(\mathrm{~m}, 1 \mathrm{H}), 2.12(\mathrm{~s}, 3 \mathrm{H}) .{ }^{13} \mathbf{C} \mathbf{N M R}\left(75 \mathbf{M H z}, \mathbf{C D C l}_{3}\right): \delta[\mathrm{ppm}]=194.1,154.4$, 151.6, 145.3, 135.4, 134.9, 116.5, 112.6, 10.54. IR (ATR): $\tilde{v}\left[\mathrm{~cm}^{-1}\right]=2816,1670,1624$, 1473, 1354, 1276, 1251, 1230, 1172, 1016, 927, 885, 744. HR-MS (EI) m/z calcd for $\left[\mathrm{C}_{8} \mathrm{H}_{8} \mathrm{O}_{2}\right][\mathrm{M}] 136.0524$ found 136.052 .<smiles>CC(C)/C(C=O)=C\c1ccco1</smiles>

(2E)-3-Methyl-2-[(furan-2-yl)methylidene]butanal (25k)

${ }^{1} \mathrm{H}$ NMR $\left(600 \mathrm{MHz}, \mathrm{CDCl}_{3}\right): \delta[\mathrm{ppm}]=9.46\left(\mathrm{~d}, 1 \mathrm{H},{ }^{4} \mathrm{~J}_{\mathrm{HH}}=1.8 \mathrm{~Hz}\right), 7.61-7.60(\mathrm{~m}, 1 \mathrm{H}), 6.85(\mathrm{~s}$, $1 \mathrm{H})$, 6.77-6.76 (m, $1 \mathrm{H}), 6.56-6.55(\mathrm{~m}, 1 \mathrm{H}), 3.53$ (sept d, $1 \mathrm{H},{ }^{3} J_{H H}=7.0 \mathrm{~Hz}{ }^{4} J_{H H}=1.8 \mathrm{~Hz}$ ), $1.30\left(\mathrm{~d}, 6 \mathrm{H},{ }^{3} \mathrm{~J}_{\mathrm{HH}}=7.0 \mathrm{~Hz}\right) .{ }^{13} \mathbf{C}$ NMR (150 MHz, $\left.\mathbf{C D C l}_{3}\right): \delta[\mathrm{ppm}]=194.7,151.2,145.1$, 
144.2, 134.8, 116.7, 112.4, 27.0, 20.0. IR (ATR) $\tilde{v}\left[\mathrm{~cm}^{-1}\right]=2962,2900,1674,1618,1456$, 1381, 1361, 1280, 1251, 1228, 1184, 1053, 1020, 931, 883. 796.<smiles>CC/C(C=O)=C\c1ccc(F)cc1</smiles>

(2E)-2-[(4-Fluorophenyl)methylidene]butanal (25h)

${ }^{1} \mathrm{H}$ NMR (600 MHz, $\left.\mathrm{CDCl}_{3}\right): \delta[\mathrm{ppm}]=9.55(\mathrm{~s}, 1 \mathrm{H}), 7.53-7.51(\mathrm{~m}, 2 \mathrm{H}), 7.18-7.14(\mathrm{~m}, 3 \mathrm{H})$, $2.56\left(\mathrm{q}, 2 \mathrm{H},{ }^{3} \mathrm{~J}_{H H}=7.5 \mathrm{~Hz}\right), 1.15\left(\mathrm{t}, 3 \mathrm{H},{ }^{3} \mathrm{~J}_{H H}=7.5 \mathrm{~Hz}\right){ }^{13} \mathrm{C} \mathrm{NMR}\left(150 \mathrm{MHz}, \mathrm{CDCl}_{3}\right): \delta[\mathrm{ppm}]=$ 195.4, $163.3\left(\mathrm{~d},{ }^{1} \mathrm{~J}_{\mathrm{CF}}=249.9 \mathrm{~Hz}\right),, 148.2,144.1,131.7,116.1,115.9,18.0,12.7$. IR (ATR): $\tilde{v}$ $\left[\mathrm{cm}^{-1}\right]=2970,1681,1597,1508,1465,1400,1226,1182,1159,1056,979,833,796$. HR-

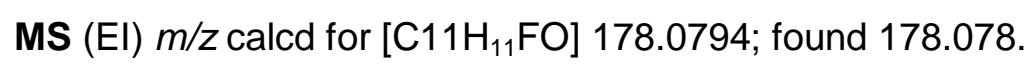

The following enals were synthesized according to ref. ${ }^{[19]}$<smiles>CC(C=O)=CCCc1ccccc1</smiles>

\section{(2E)-2-Methyl-5-phenylpent-2-enal (25e)}

${ }^{1} \mathrm{H}$ NMR (300 MHz, $\left.\mathbf{C D C l}_{3}\right): \delta[\mathrm{ppm}]=9.38(\mathrm{~s}, 1 \mathrm{H}), 7.33-7.18(\mathrm{~m}, 5 \mathrm{H}), 6.50\left(\mathrm{t},{ }^{3} \mathrm{~J}_{H H}=7.2 \mathrm{~Hz}\right.$, $1 \mathrm{H}), 2.82\left(\mathrm{t},{ }^{3} \mathrm{~J}_{H H}=7.5 \mathrm{~Hz}, 2 \mathrm{H}\right), 2.68(\mathrm{~m}, 2 \mathrm{H}), 1.68(\mathrm{~s}, 3 \mathrm{H}) .{ }^{13} \mathbf{C} \mathbf{N M R}\left(75 \mathbf{M H z}, \mathbf{C D C l}_{3}\right): \delta$ $[\mathrm{ppm}]=195.07,153.01,140.6,139.9,128.5,128.3,126.3,34.4,30.6,9.13$. IR (ATR): $\tilde{v}$ $\left[\mathrm{cm}^{-1}\right]=2924,2858,2818,2711,1681,1643,11494,1454,1240,1014,, 746,698$. HR-MS (EI) $\mathrm{m} / \mathrm{z}$ calcd for $\left[\mathrm{C}_{12} \mathrm{H}_{14} \mathrm{O}\right][\mathrm{M}] 174.1045$ found 174.104 .<smiles>O=C/C=C/c1ccc(Cl)cc1</smiles>

(2E)-3-(4-Chlorophenyl)-2-propenal (25t)

${ }^{1} \mathrm{H}$ NMR $\left(600 \mathrm{MHz}, \mathrm{CDCl}_{3}\right): \delta[\mathrm{ppm}]=9.70(\mathrm{~s}, 1 \mathrm{H}), 7.52-7.40(\mathrm{~m}, 5 \mathrm{H}), 6.68\left(\mathrm{dd}, 1 \mathrm{H},{ }^{3} \mathrm{~J}_{H H}=\right.$ $15.2 \mathrm{~Hz}) .{ }^{13} \mathrm{C}$ NMR (150 MHz, $\left.\mathbf{C D C l}_{3}\right): \delta[\mathrm{ppm}]=193.20,150.86,137.28,132.54,129.58$, 129.42, 129.0. IR (ATR): $\tilde{v}$ [ $\left.\mathrm{cm}^{-1}\right]: 2852,1693,1670,1625,1589,1490,1411,1396,1300$, $1288,1255,1124,1085,1004,975,858,806,682$. 


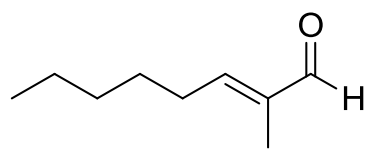

(2E)-2-Methyloct-2-enal (25f)

${ }^{1} \mathbf{H}$ NMR (300 MHz, $\left.\mathbf{C D C l}_{3}\right): \delta[p p m]=9,40(\mathrm{~s}, 1 \mathrm{H}), 6,52-6.47(\mathrm{~m}, 1 \mathrm{H}), 2,39-2.31(\mathrm{~m}, 2 \mathrm{H})$, $1,75\left(\mathrm{~s}, 3 \mathrm{H}\right.$ ), 1,56-1.46 (quin, ${ }^{3} \mathrm{~J}_{H H}=7,1 \mathrm{~Hz}$ ), 1,35-1.31(m, 4H), 0,91 (t, $\left.{ }^{3} \mathrm{~J}_{H H}=6,6 \mathrm{~Hz}\right) .{ }^{13} \mathrm{C}$ NMR (75 MHz, $\left.\mathbf{C D C l}_{3}\right): \delta[p p m]=195.3,154.9,139.3,31.5,28.9,28.04,22.4,13.9,9.12$. IR (ATR): $\tilde{v}\left[\mathrm{~cm}^{-1}\right]=2956,2927,2858,1685,1645,1460,1238,1070,999$.

The following enals were synthesized according to ref. ${ }^{[20]}$<smiles>CC/C(C=O)=C(/C)c1ccccc1</smiles>

(2E)-2-Methyl-3-phenylpent-2-enal (25w)

${ }^{1} \mathbf{H}$ NMR $\left(600 \mathrm{MHz}, \mathbf{C D C l}_{3}\right): \delta[\mathrm{ppm}]=10.3(\mathrm{~s}, 1 \mathrm{H}), 7.43-7.33(\mathrm{~m}, 3 \mathrm{H}), 7.17-7.14(\mathrm{~m}, 2 \mathrm{H})$, $2.92\left(\mathrm{q}, 2 \mathrm{H},{ }^{3} \mathrm{~J}_{\mathrm{HH}}=7.4 \mathrm{~Hz}\right), 1.62(\mathrm{~s}, 3 \mathrm{H}), 1.06\left(\mathrm{t}, 3 \mathrm{H},{ }^{3} \mathrm{~J}_{\mathrm{HH}}=7.4 \mathrm{~Hz}\right) .{ }^{13} \mathrm{C}$ NMR (150 MHz, $\left.\mathrm{CDCl}_{3}\right): \delta[\mathrm{ppm}]=191.73,161.9,141.4,128.3,127.8,127.3,25.9,14.3,13.0$.<smiles>C/C(C=O)=C\c1ccccc1</smiles>

\section{(2E)-2-Methyl-3-phenylbut-2-enal (9)}

${ }^{1} \mathrm{H}$ NMR $\left(600 \mathrm{MHz}, \mathrm{CDCl}_{3}\right): \delta[\mathrm{ppm}]=9.45(\mathrm{~s}, 1 \mathrm{H}), 7.38-7.35(\mathrm{~m}, 3 \mathrm{H}), 7.21-7.20(\mathrm{~m}, 2 \mathrm{H})$, $2.28(\mathrm{~s}, 3 \mathrm{H}), 1.91$ (s, 3H). ${ }^{13} \mathrm{C}$ NMR (150 MHz, $\left.\mathbf{C D C l}_{3}\right): \delta[\mathrm{ppm}]=193.7,157.6,140.2,134.3$, $128.8,128.24,128.23,23.5,11.2$.

The following enal was synthesized according to ref. ${ }^{[21]}$<smiles>CC(C=O)=C(c1ccccc1)c1ccccc1</smiles>

\section{2-Methyl-3,3-diphenylpropenal (25x)}

${ }^{1} \mathrm{H}$ NMR (600 MHz, $\left.\mathbf{C D C l}_{3}\right): \delta[\mathrm{ppm}]=9.6(\mathrm{~s}, 1 \mathrm{H}), 7.40-7.34(\mathrm{~m}, 6 \mathrm{H}, \mathrm{Ar}), 7.19-7.16(\mathrm{~m}, 4 \mathrm{H}$, $\operatorname{Ar}), 1.97$ (s, 3H). ${ }^{13} \mathrm{C}$ NMR (150 MHz, $\left.\mathbf{C D C l}_{3}\right): \delta[\mathrm{ppm}]=194.4,159.4,140.8,138.9,135.3$, 131.2, 129.6, 128.9, 128.6, 128.3, 128.1, 14.3. 
The enal $25 q$ was synthesized according to ref. ${ }^{[22]}$<smiles>CCOC(=O)/C(=C/C=O)c1ccccc1</smiles>

\section{Ethyl (E)-4-oxo-2-phenylbut-2-enoate (25q)}

${ }^{1} \mathrm{H}$ NMR $\left(600 \mathrm{MHz}, \mathrm{CDCl}_{3}\right): \delta[\mathrm{ppm}]=9.65\left(\mathrm{~d}, 1 \mathrm{H},{ }^{3} \mathrm{JHH}_{\mathrm{HH}}=7.8 \mathrm{~Hz}\right), 7.49-7.44(\mathrm{~m}, 3 \mathrm{H}, \mathrm{Ar})$, 7.38-7.36 (m, 2H, Ar), $7.0\left(\mathrm{~d}, 1 \mathrm{H},{ }^{3} \mathrm{JHH}_{\mathrm{HH}}=7.8 \mathrm{~Hz}\right), 4.34\left(\mathrm{q}, 2 \mathrm{H},{ }^{3} \mathrm{JHH}_{\mathrm{HH}}=7.8 \mathrm{~Hz}\right), 1.35\left(\mathrm{t}, 3 \mathrm{H},{ }^{3} \mathrm{JHH}_{\mathrm{HH}}\right.$ $=7.8 \mathrm{~Hz}) .{ }^{13} \mathrm{C} \mathrm{NMR}\left(150 \mathrm{MHz}, \mathrm{CDCl}_{3}\right): \delta[\mathrm{ppm}]=193.5,166.5,149.5,134.6,130.5,129.7$, 128.5, 62.3, 14.1 .

The enal $25 \mathrm{~m}$ was synthesized according to ref. ${ }^{[23]}$<smiles>COc1ccc(/C=C/C(=O)F)cc1</smiles>

(Z/E)-2-fluoro-3-(4-methoxyphenyl)acrylaldehyde (25m)

${ }^{1} \mathrm{H} \mathrm{NMR}\left(600 \mathrm{MHz}, \mathrm{CDCl}_{3}\right): \delta[\mathrm{ppm}]=9.67\left(\mathrm{~d}, 1 \mathrm{H},{ }^{3} \mathrm{JHH}_{\mathrm{HH}}=19.4 \mathrm{~Hz}\right), 7.34-7.32(\mathrm{~m}, 2 \mathrm{H}, \mathrm{Ar})$, 6.96-6.95 (m, 2H, Ar),3.85 (s, 3H). ${ }^{13} \mathrm{C}$ NMR (150 MHz, $\left.\mathrm{CDCl}_{3}\right): \delta[p p m]=183.8\left(\mathrm{~d},{ }^{2} \mathrm{~J}_{\mathrm{CF}}=24\right.$ $\mathrm{Hz}), 161.2,154.1\left(\mathrm{~d},{ }^{1} J_{\mathrm{CF}}=254 \mathrm{~Hz}\right), 131.5\left(\mathrm{~d},{ }^{4} J_{\mathrm{CF}}=2.6 \mathrm{~Hz}\right), 127.7\left(\mathrm{~d},{ }^{2} J_{\mathrm{CF}}=22.4 \mathrm{~Hz}\right), 122.2$ $\left(d,{ }^{3} J_{C F}=9.42 \mathrm{~Hz}\right), 114.4,55.4$

\section{General procedure for the redox esterification of enals}<smiles>[R]C([R])=C([R])C=O</smiles>

25

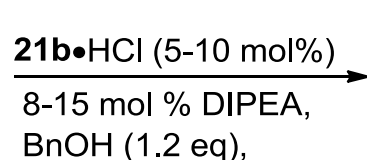
$100{ }^{\circ} \mathrm{C}$<smiles>[R]C([R])C([R])C(=O)OCc1ccccc1</smiles>
26

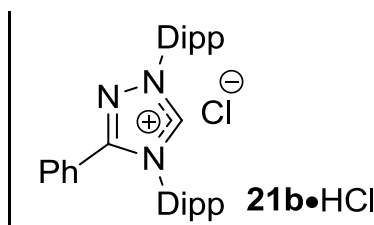

Dipp 21b•HC

In a glove box, a screw-capped GC vial was charged with the triazolium salt $\mathbf{2 1 \mathbf { b }} \cdot \mathbf{H C l}(0.05$ to 0.1 equiv), benzyl alcohol (1.2 equiv), aldehyde (1.0 equiv) and DIPEA (0.08-0.15 equiv). The reaction mixture was stirred at $100{ }^{\circ} \mathrm{C}$ until aldehyde was completely consumed (analysis by GC). The reaction mixture was cooled to room temperature, and the product was purified by flash column chromatography on silica gel. 


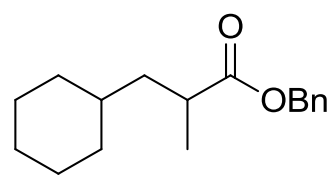

\section{Benzyl 3-cyclohexyl-2-methylpropanoate (26a)}

${ }^{1} \mathrm{H}$ NMR (300 MHz, $\left.\mathbf{C D C l}_{3}\right): \delta[p p m]=7.37-7.33(\mathrm{~m}, 5 \mathrm{H}), 5.14\left(\mathrm{~d}, 1 \mathrm{H},{ }^{3} \mathrm{~J}_{H H}=12.3 \mathrm{~Hz}\right), 5.09$ $\left(\mathrm{d}, 1 \mathrm{H},{ }^{3} J_{H H}=12.3 \mathrm{~Hz}\right), 2.64-2.56(\mathrm{~m}, 1 \mathrm{H}), 1.76-1.57(\mathrm{~m}, 6 \mathrm{H}),, 1.24-1.11(\mathrm{~m}, 8 \mathrm{H}),, 0.90-0.81$ $(\mathrm{m}, 2 \mathrm{H}) .{ }^{13} \mathrm{C}$ NMR $\left(75 \mathrm{MHz}, \mathbf{C D C l}_{3}\right): \delta[\mathrm{ppm}]=177.0,136.3,128.5,128.1,65.9,41.6,36.9$, 35.4, 33.2, 26.5, 26.2, 17.6.. IR (ATR): $\tilde{v}\left[\mathrm{~cm}^{-1}\right]=2968,2922,2850,1732,1448,1381$, 1352, 1311, 1246, 1211, 1188, 1161, 1149, 1132, 1076, 1043, 1022, 966, 902, 813, 748, 732, 696. HR-MS (EI) $\mathrm{m} / \mathrm{z}$ calcd for $\left[\mathrm{C}_{17} \mathrm{H}_{24} \mathrm{O}_{2}\right]$ [M] 260.1776 found 260.176 .<smiles>CC(Cc1ccccc1)C(=O)OCc1ccccc1</smiles>

\section{Benzyl 2-benzylpropanoate (26b)}

${ }^{1} \mathrm{H}$ NMR (600 MHz, $\left.\mathbf{C D C l}_{3}\right): \delta[\mathrm{ppm}]=7.39-7.33(\mathrm{~m}, 3 \mathrm{H}), 7.30-7.28(\mathrm{~m}, 4 \mathrm{H}), 7.25-7.22(\mathrm{~m}$, $1 \mathrm{H}), 7.18-7.17(\mathrm{~m}, 2 \mathrm{H}), 5.11(\mathrm{~s}, 2 \mathrm{H}), 3.07\left(\mathrm{dd},{ }^{3} J_{H H}=7.2 \mathrm{~Hz},{ }^{2} J_{H H}=13.5 \mathrm{~Hz}, 1 \mathrm{H}\right), 2.84(\mathrm{~m}$, $1 \mathrm{H}), 2.73\left(\mathrm{dd},{ }^{2} J_{H H}=13.5 \mathrm{~Hz},{ }^{3} J_{H H}=7.6 \mathrm{~Hz}, 1 \mathrm{H}\right), 1.22\left(\mathrm{~d},{ }^{3} J_{H H}=6.9 \mathrm{~Hz}, 3 \mathrm{H}\right) .{ }^{13} \mathrm{C}$ NMR (150 $\left.\mathbf{M H z}, \mathbf{C D C l}_{3}\right): \delta[\mathrm{ppm}]=175.84,139.27,136.08,128.98,128.48,128.35,128.26,128.07$, 128.05, 126.30, 66.11, 41.50, 39.76, 16.80. IR (ATR): $\tilde{v}\left[\mathrm{~cm}^{-1}\right]=2956,1732,1454,1381$, 1251, 1209, 1153, 1066, 1028, 871, 840, 742, 731, 696. HR-MS (EI) m/z calcd for $\left[\mathrm{C}_{17} \mathrm{H}_{18} \mathrm{O}_{2}\right][\mathrm{M}] 254.1307$ found 254.131.<smiles>CC(Cc1ccco1)C(=O)OCc1ccccc1</smiles>

\section{Benzyl 3-(furan-2-yl)-2-methylpropanoate (26c)}

${ }^{1} \mathrm{H}$ NMR (600 MHz, $\left.\mathbf{C D C l}_{3}\right): \delta[\mathrm{ppm}]=7.38-7.37(\mathrm{~m}, 2 \mathrm{H}), 7.35-7.34(\mathrm{~m}, 3 \mathrm{H}), 7.31-7.30(\mathrm{~m}$, $1 \mathrm{H}), 6.28-6.27(\mathrm{~m}, 1 \mathrm{H}), 6.03-6.02(\mathrm{~m} \mathrm{1H}), 5.14(\mathrm{~s}, 2 \mathrm{H}), 3.07\left(\mathrm{dd},{ }^{3} J_{H H}=7.0 \mathrm{~Hz},{ }^{2} J_{H H}=14.8\right.$ $\mathrm{Hz}, 1 \mathrm{H}), 2.91(\mathrm{~m}, 1 \mathrm{H}) 2.79\left(\mathrm{dd},{ }^{2} J_{H H}=14.8 \mathrm{~Hz},{ }^{3} J_{H H}=7.2 \mathrm{~Hz}, 1 \mathrm{H}\right), 2.0-1.98(\mathrm{~m}, 1 \mathrm{H}), 1.23(\mathrm{~d}$, $\left.{ }^{3} J_{H H}=7.0 \mathrm{~Hz}, 3 \mathrm{H}\right){ }^{13} \mathrm{C}$ NMR $\left(150 \mathrm{MHz}, \mathbf{C D C l}_{3}\right): \delta[\mathrm{ppm}]=175.50,153.15,141.33,136.04$, 128.53, 128.16, 128.13, 110.16, 106.50, 66.30, 39.0. IR (ATR): $\tilde{v}\left[\mathrm{~cm}^{-1}\right]=2974,2937,1730$, 1456, 1382, 1352, 1159, 1082, 1008, 966, 804, 734, 696. HR-MS (EI) m/z calcd for $\left[\mathrm{C}_{15} \mathrm{H}_{16} \mathrm{O}_{3}\right][\mathrm{M}] 244.1099$ found 244.110 . 
<smiles>CCCC(C)C(=O)OCc1ccccc1</smiles>

\section{Benzyl 2-methylpentanoate (26d)}

${ }^{1} \mathrm{H}$ NMR (600 MHz, $\left.\mathrm{CDCl}_{3}\right): \delta[\mathrm{ppm}]=7.40-7.33(\mathrm{~m}, 5 \mathrm{H}), 5.15(\mathrm{~s}, 2 \mathrm{H}), 2.54(\mathrm{~m}, 1 \mathrm{H}), 1.73-$ $1.67(\mathrm{~m}, 1 \mathrm{H}), 1.47-1.41(\mathrm{~m}, 1 \mathrm{H}), 1.37-1.30(\mathrm{~m}, 2 \mathrm{H}), 1.20\left(\mathrm{~d},{ }^{3} J_{H H}=7.0 \mathrm{~Hz}, 3 \mathrm{H}\right), 0.92\left(\mathrm{t},{ }^{3} J_{H H}=\right.$ $7.3 \mathrm{~Hz}, 3 \mathrm{H}) .{ }^{13} \mathrm{C}$ NMR (150 MHz, $\left.\mathbf{C D C l}_{3}\right): \delta[\mathrm{ppm}]=176.7,136.3,128.5,128.1,128.0,66.0$, 39.3, 35.9, 20.4, 17.0, 13.9. . IR (ATR): $\tilde{v}\left[\mathrm{~cm}^{-1}\right]=2958,2933,2873,1732,1456,1381$, 1240, 1170, 1141, 1082, 1028, 966, 910, 734, 696. HR-MS (El) m/z calcd for $\left[\mathrm{C}_{13} \mathrm{H}_{18} \mathrm{O}_{2}\right]^{+}$ $[\mathrm{M}]^{+} 206.1307$ found 206.131 .<smiles>CC(CCCc1ccccc1)C(=O)OCc1ccccc1</smiles>

\section{Benzyl 2-methyl-5-phenylpentanoate (26e)}

${ }^{1} \mathrm{H}$ NMR (300 MHz, $\left.\mathbf{C D C l}_{3}\right): \delta[\mathrm{ppm}]=7.33-7.11(\mathrm{~m}, 10 \mathrm{H}), 5.11(\mathrm{~s}, 2 \mathrm{H}), 2.60-2.48(\mathrm{~m}, 3 \mathrm{H})$, $1,77-1.43(\mathrm{~m}, 4 \mathrm{H}), 1.16(\mathrm{~d}, \mathrm{~J}=7.0 \mathrm{~Hz}, 3 \mathrm{H}) .{ }^{13} \mathbf{C ~ N M R}\left(75 \mathbf{M H z}, \mathbf{C D C l}_{3}\right): \delta[\mathrm{ppm}]=176,41$, $142,1,136,3,128.5$ 128.3, 128.25, 128.1, 128.05, 125.7, 66.0, 39.4, 35.7 , 33.4, 29.0, 17.0. IR (ATR): $\tilde{v}\left[\mathrm{~cm}^{-1}\right]=3028,2972,2935,2860,1732,1496,1454,1382,1352,1253,1221$, 1147, 1120, 1078,21 1053, 1029, 1002, 964, 906, 746, 696. HR-MS (El) m/z calcd for $\left[\mathrm{C}_{19} \mathrm{H}_{22} \mathrm{O}_{2}\right][\mathrm{M}] 282.1620$ found 282.3161.

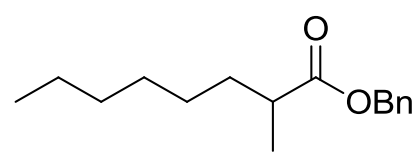

\section{Benzyl 2-methyloctanoate (26f)}

${ }^{1} \mathbf{H}$ NMR (300 MHz, $\left.\mathbf{C D C l}_{3}\right): \delta[p p m]=7.36-7.32(\mathrm{~m}, 5 \mathrm{H}), 5.11(\mathrm{~s}, 2 \mathrm{H}), 2.54-2.43(\mathrm{~m}, 1 \mathrm{H})$, 1.28-1.25 (m, 9H), 1,17-1.15 (m, 3H), 0.89-0.84 (m, 4H,). ${ }^{13} \mathbf{C}$ NMR (150 MHz, CDCl $\left.\mathbf{~}_{3}\right): \delta$ $[\mathrm{ppm}]=176.6,136.3,128.5,128.02,65.9,39.6,33.8,31.6 .29 .1 .27 .1,22.5 .17 .0,14.0$. IR (ATR): $\tilde{v}\left[\mathrm{~cm}^{-1}\right]=3089,3066,3034,2954,2927,2856,1734,1691,1498,1456,1381$, 1352, 1253, 1242, 1211, 1163, 1139, 1107, 1080, 1056, 1028, 966, 906 , 885, 804, 748, 732, 696. HR-MS (EI) $m / z$ calcd for $\left[\mathrm{C}_{16} \mathrm{H}_{24} \mathrm{O}_{2}\right]$ [M] 248.1776 found 248.176 .<smiles>CCC(Cc1ccccc1)C(=O)OCc1ccccc1</smiles>

Benzyl 2-benzylbutanoate (26g) 
${ }^{1} \mathbf{H}$ NMR (600 MHz, $\left.\mathbf{C D C l}_{3}\right): \delta[\mathrm{ppm}]=7.31-7.12(\mathrm{~m}, 10 \mathrm{H}), 5.03(\mathrm{~s}, 2 \mathrm{H}), 2.97-2.90(\mathrm{~m}, 1 \mathrm{H})$, 2.80-2.73 (m, 1H), 2.70-2.50 (m, 1H), 1.76-1.50 (m, 2H), $0.90\left(\mathrm{t},{ }^{3} \mathrm{~J}_{H H}=7.4 \mathrm{~Hz}, 3 \mathrm{H}\right){ }^{13} \mathrm{C} \mathrm{NMR}$ $\left(150 \mathrm{MHz}, \mathrm{CDCl}_{3}\right): \delta[\mathrm{ppm}]=175.33,139.42,136.1,128.91,128.46,128.38,128.12,128.05$, 126.3, 66.0, 49.24, 38.20, 25.25, 11.72, IR (ATR): $\tilde{v}\left[\mathrm{~cm}^{-1}\right]=3030,2964,1730,1496,1454$, $1384,1357,1263,1151,1072,1029,740,696$. HR-MS (EI) $\mathrm{m} / z$ calcd for $\left[\mathrm{C}_{18} \mathrm{H}_{20} \mathrm{O}_{2}\right][\mathrm{M}]$ 268.1463 found 268.146 .<smiles>CCC(Cc1ccc(F)cc1)C(=O)OCc1ccccc1</smiles>

\section{Benzyl 2-[(4-fluorophenyl)methyl]butanoate (26h)}

${ }^{1} \mathbf{H}$ NMR (600 MHz, $\left.\mathbf{C D C l}_{3}\right): \delta[\mathrm{ppm}]=7.36-7.33(\mathrm{~m}, 3 \mathrm{H}), 7.23-7.22(\mathrm{~m}, 2 \mathrm{H}), 7.11-7.09(\mathrm{~m}$, $2 \mathrm{H}), 6.95-6.92(\mathrm{~m}, 2 \mathrm{H}), 5.1\left(\mathrm{~d}, 1 \mathrm{H},{ }^{2} J_{H H}=12.3 \mathrm{~Hz}\right), 5.05\left(\mathrm{~d}, 1 \mathrm{H},{ }^{2} J_{H H}=12.3 \mathrm{~Hz}\right), 2.92(\mathrm{dd}$, $\left.{ }^{2} J_{H H}=13.8 \mathrm{~Hz},{ }^{3} J_{H H}=9.0 \mathrm{~Hz}\right), 2.78\left(\mathrm{dd},{ }^{2} J_{H H}=13.8 \mathrm{~Hz},{ }^{3} J_{H H}=6.2 \mathrm{~Hz}\right), 2.67-2.62(\mathrm{~m}, 1 \mathrm{H})$, 1.76-1.68 (m, 1H), 1.65-1.60 (m, 1H), $0.95\left(\mathrm{t},{ }^{3} \mathrm{~J}=7.4 \mathrm{~Hz}\right) .{ }^{13} \mathbf{C}$ NMR (150 MHz, $\left.\mathbf{C D C l}_{3}\right): \delta$ $[p p m]=175.21,161.7\left(\mathrm{~d},{ }^{1} J_{\mathrm{CF}}=242.5 \mathrm{~Hz}\right), 135.95,135.01,134.99,130.32,130.27,128.49$, 128.19, 128.15, 115.57, 115.21, 66.04, 49.43, 37.38, 25.33, 11.75. IR (ATR): $\tilde{v}\left[\mathrm{~cm}^{-1}\right]=$ 2964, 2933, 1730, 1600, 1508, 1456, 1220, 1153, 975, 833, 748, 696. HR-MS (El) m/z calcd for $\left[\mathrm{C}_{18} \mathrm{H}_{19} \mathrm{FO}_{2}\right][\mathrm{M}] 286.1369$ found 286.137 .<smiles>CCC(Cc1cccs1)C(=O)OCc1ccccc1</smiles>

\section{benzyl 2-[(thiophen-2-yl)methyl]butanoate (26i)}

${ }^{1} \mathbf{H}$ NMR (600 MHz, $\left.\mathbf{C D C l}_{3}\right): \delta[\mathrm{ppm}]=7.39-7.35(\mathrm{~m}, 3 \mathrm{H}), 7.32-7.31(\mathrm{~m}, 2 \mathrm{H}), 7.16-7.15(\mathrm{~m}$, $1 \mathrm{H})$, 6.93-6.92 (m, $1 \mathrm{H}), 6.81-6.80(\mathrm{~m}, 1 \mathrm{H}), 5.14(\mathrm{~s}, 2 \mathrm{H}), 3.25-3.20(\mathrm{~m}, 1 \mathrm{H}), 3.05-3.01(\mathrm{~m}$, 1H), 2.75-2.73 (m, 1H), 1.76-1.66 (m, 2H), 0.98-0.95 (m, 3H). ${ }^{13} \mathbf{C}$ NMR (150 MHz, $\left.\mathbf{C D C l}_{3}\right): \delta$ $[\mathrm{ppm}]=174.98,141.72,136.01,128.52,128.20,128.15,126.81,125.52,123.73,66.22$, 49.50, 31.96, 25.13, 11.63. IR (ATR): $\tilde{v}\left[\mathrm{~cm}^{-1}\right]=2964,2933,2875,1730,1456,1438,1384$, 1259, 1199, 1165, 1089, 1074, 904, 827, 790, 734, 694. HR-MS (El) m/z calcd for $\left[\mathrm{C}_{16} \mathrm{H}_{18} \mathrm{O}_{2} \mathrm{~S}\right]^{+}[\mathrm{M}]^{+} 234.1620$ found 234.161. 


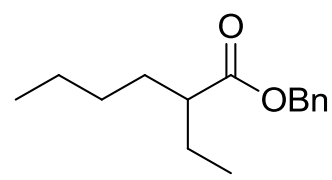

Benzyl 2-methylpentanoate (26j)

${ }^{1} \mathrm{H}$ NMR (600 MHz, $\left.\mathrm{CDCl}_{3}\right): \delta[\mathrm{ppm}]=7.36-7.35(\mathrm{~m}, 5 \mathrm{H}), 5.13(\mathrm{~s}, 2 \mathrm{H}), 2.34-2.30(\mathrm{~m}, 1 \mathrm{H})$, 1.67- $1.60(\mathrm{~m}, 2 \mathrm{H}), 1.56-1.44(\mathrm{~m}, 2 \mathrm{H}), 1.32-1.20(\mathrm{~m}, 4 \mathrm{H}), 0.90-0.84(\mathrm{~m}, 6 \mathrm{H}) .{ }^{13} \mathrm{C}$ NMR (150 $\left.\mathbf{M H z}, \mathbf{C D C l}_{3}\right): \delta[\mathrm{ppm}]=176.27,136.35,128.51,128.16,128.10,65.87,47.34,31.81,29.60$, 25.51, 22.63, 14.0, 11.8. IR (ATR): $\tilde{v}\left[\mathrm{~cm}^{-1}\right]=2958,2933,1732,1496,1456,1381,1265$, $1213,1163,1139,975,746,732,696$. HR-MS (EI) $\mathrm{m} / z$ calcd for $\left[\mathrm{C}_{15} \mathrm{H}_{22} \mathrm{O}_{2}\right]^{+}[\mathrm{M}]^{+} 234.1620$ found 234.161 .<smiles>CC(C)C(Cc1ccco1)C(=O)OCc1ccccc1</smiles>

benzyl 2-[(furan-2-yl)methyl]-3-methylbutanoate (26k)

${ }^{1} \mathrm{H}$ NMR (600 MHz, $\left.\mathrm{CDCl}_{3}\right): \delta[\mathrm{ppm}]=7.37-7.28(\mathrm{~m}, 6 \mathrm{H}), 6.26-6.25(\mathrm{~m} \mathrm{1H})$, 5.99-5.98 (m, $1 \mathrm{H}), 5.11(\mathrm{~s}, 2 \mathrm{H}), 3.01\left(\mathrm{dd},{ }^{3} J_{H H}=10.4 \mathrm{~Hz},{ }^{2} J_{H H}=15 \mathrm{~Hz}, 1 \mathrm{H}\right), 2.87\left(\mathrm{dd},{ }^{3} J_{H H}=4.5 \mathrm{~Hz},{ }^{2} J_{H H}=\right.$ $15 \mathrm{~Hz}, 1 \mathrm{H}), 2.68-2.64(\mathrm{~m}, 1 \mathrm{H}), 2.02-1.94(\mathrm{~m} 1 \mathrm{H}), 1.03\left(\mathrm{~d}, 3 \mathrm{H},{ }^{3} J_{H H}=6.8 \mathrm{~Hz}\right), 0.98(\mathrm{~d}, 3 \mathrm{H}$, $\left.{ }^{3} J_{H H}=6.8 \mathrm{~Hz}\right) .{ }^{13} \mathrm{C}$ NMR $\left(150 \mathrm{MHz}, \mathrm{CDCl}_{3}\right): \delta[\mathrm{ppm}]=174.52,153.6,141.2,136.0,128.5$, 128.3, 128.1, 110.2, 106.0, 66.1, 51.5, 30.5, 28.2, 20.2, 20.06.

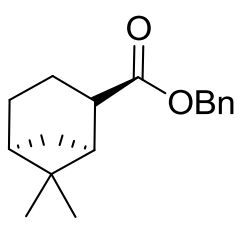

major

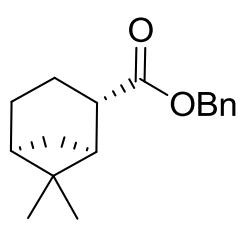

minor

\section{Benzyl 6,6-dimethylbicyclo[3.1.1]heptane-2-carboxylate (26I)}

These diastereomers did not separated by column chromatography. The diastereomeric ratio major:minor (2:1) was predicted according to NOE spectrum. Major isomer: ${ }^{1} \mathbf{H}$ NMR (600 $\left.\mathbf{M H z}, \mathbf{C D C l}_{3}\right): \delta[\mathrm{ppm}]=7.35-7.33(\mathrm{~m}, 5 \mathrm{H}), 5.12\left(\mathrm{~d}, 1 \mathrm{H},{ }^{3} \mathrm{~J}=12.5 \mathrm{~Hz}\right), 5.09\left(\mathrm{~d}, 1 \mathrm{H},{ }^{3} \mathrm{~J}=12.5\right.$ $\mathrm{Hz}), 2.98-2.94(\mathrm{~m}, 1 \mathrm{H}), 2.52-2.51(\mathrm{~m}, 1 \mathrm{H}), 2.34-2.28(\mathrm{~m}, 2 \mathrm{H}), 1.98-1.83(\mathrm{~m}, 4 \mathrm{H}), 1.17-1.15$ $(\mathrm{m}, 4 \mathrm{H}), 0.74(\mathrm{~s}, 3 \mathrm{H}) .{ }^{13} \mathrm{C}$ NMR (150 MHz, $\left.\mathbf{C D C l}_{3}\right): \delta[\mathrm{ppm}]=176.24,136.31,128.48,128.07$, 128.04, 66.09, 44.05, 43.12, 40.41, 38.71, 29.07, 27.02, 24.74, 21.55, 15.37. Minor isomer: ${ }^{13} \mathrm{C}$ NMR (150 MHz, $\left.\mathrm{CDCl}_{3}\right): \delta[\mathrm{ppm}]=176.46,136.34,128.54,128.09,128.06,66.09$, 43.72, 41.3, 40.15, 39.20, 26.45, 24.20, 23.8, 20.29, 16.87. IR (ATR): $\tilde{v}\left[\mathrm{~cm}^{-1}\right]=2947,2918$, 1730, 1456, 1382, 1367, 1172, 1143, 1006, 983, 748, 732.696. HR-MS (EI) m/z calcd for $\left[\mathrm{C}_{1} \mathrm{H}_{22} \mathrm{O}_{2}\right]^{+}[\mathrm{M}]^{+} 258.162$ found 258.161 . 


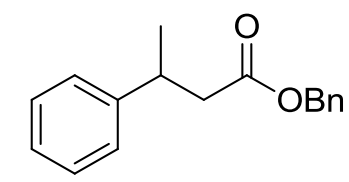

\section{Benzyl 3-phenylbutanoate (26n)}

${ }^{1} \mathrm{H}$ NMR (600 MHz, $\left.\mathrm{CDCl}_{3}\right): \delta[\mathrm{ppm}]=7.4-7.28(\mathrm{~m}, 5 \mathrm{H}), 7.26-7.20(\mathrm{~m}, 5 \mathrm{H}), 5.07(\mathrm{~s}, 2 \mathrm{H}), 3.32$ $(\mathrm{m}, 1 \mathrm{H}), 2.70\left(\mathrm{dd},{ }^{3} \mathrm{~J}_{\mathrm{HH}}=7.26 \mathrm{~Hz},{ }^{2} J_{\mathrm{HH}}=15.12 \mathrm{~Hz}, 1 \mathrm{H}\right), 2.64-2.60(\mathrm{~m}, 1 \mathrm{H}), 1.31\left(\mathrm{~d}, 3 \mathrm{H},{ }^{3} \mathrm{~J}_{\mathrm{HH}}=\right.$ $6.9 \mathrm{~Hz}) .{ }^{13} \mathrm{C}$ NMR (150 MHz, $\left.\mathbf{C D C l}_{3}\right): \delta[\mathrm{ppm}]=172.2,145.6,135.9,128.56,128.54,128.17$, 126.8, 126.46, 66.2, 42.9, 36.6, 21.9.<smiles>CC(C)=CCCC(C)CC(=O)OCc1ccccc1</smiles>

\section{Benzyl 3,7-dimethyloct-6-enoate (26o)}

${ }^{1} \mathrm{H}$ NMR (600 MHz, $\left.\mathrm{CDCl}_{3}\right): \delta[\mathrm{ppm}]=7.39-7.34(\mathrm{~m}, 5 \mathrm{H}), 5.14(\mathrm{~s}, 2 \mathrm{H}), 5.11-5.08(\mathrm{~m}, 1 \mathrm{H})$, $2.39\left(\mathrm{dd},{ }^{2} J_{H H}=14.8 \mathrm{~Hz},{ }^{3} J_{H H}=6.0 \mathrm{~Hz}, 1 \mathrm{H}\right), 2.19\left(\mathrm{dd},{ }^{2} J_{H H}=14.8 \mathrm{~Hz},{ }^{3} J_{H H}=8.2 \mathrm{~Hz}, 1 \mathrm{H}\right)$, 2.04-1.96 (m, 3H), $1.70(\mathrm{~s}, 3 \mathrm{H}), 1.61(\mathrm{~s}, 3 \mathrm{H}), 1.39-1.36(\mathrm{~m}, 1 \mathrm{H}), 1.27-1.24(\mathrm{~m}, 1 \mathrm{H}), 0.96(\mathrm{~d}$, $\left.{ }^{3} \mathrm{~J}_{H H}=6.6 \mathrm{~Hz}, 3 \mathrm{H}\right) .{ }^{13} \mathrm{C}$ NMR (150 MHz, $\left.\mathbf{C D C l}_{3}\right): \delta[\mathrm{ppm}]=173.10,136.16,131.55,128.54$, 128.21, 128.16, 124.26, 66.04, 41.80, 36.76, 30.07, 25.71, 25.41, 19.63, 17.65. IR (ATR): $\tilde{v}$ $\left[\mathrm{cm}^{-1}\right]=2960,1730,1456,1379,1259,1157,975,910,738,696$. HR-MS (EI) $\mathrm{m} / \mathrm{z}$ calcd for $\left[\mathrm{C}_{17} \mathrm{H}_{24} \mathrm{O}_{2}\right][\mathrm{M}] 260.1776$ found 260.171 .

\section{$\overbrace{\mathrm{OBn}}^{\mathrm{O}}$ \\ Benzyl 3-methylbutanoate (26p)}

${ }^{1} \mathbf{H}$ NMR (600 MHz, $\left.\mathbf{C D C l}_{3}\right): \delta[\mathrm{ppm}]=7.36-7.30(\mathrm{~m} .5 \mathrm{H}) .5 .11(\mathrm{~s}, 2 \mathrm{H}), 2.25-2.22(\mathrm{~m}, 2 \mathrm{H})$, 2.18-2.07 (m, 1H), $0.94\left(\mathrm{~d},{ }^{3} \mathrm{~J}_{\mathrm{HH}}=6.5 \mathrm{~Hz}, 6 \mathrm{H}\right) .{ }^{13} \mathrm{C}$ NMR (150 MHz, $\left.\mathbf{C D C l}_{3}\right): \delta[\mathrm{ppm}]=$ 172.90, 136.20, 128.51, 128.15, 128.11, 65.96, 43.41, 25.71, 22.37. IR (ATR) $\tilde{v}$ [cm ${ }^{-1}$ ]: 2958, 2933, 1732, 1456, 1377, 1292, 1255, 1182, 1165, 1118, 1095, 1001, 736, 696.

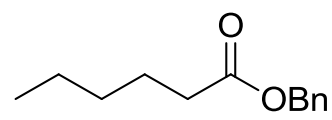

\section{Benzyl hexanoate (26r)}

${ }^{1} \mathrm{H}$ NMR $\left(600 \mathrm{MHz}, \mathrm{CDCl}_{3}\right): \delta[\mathrm{ppm}]=7.38-7.34(\mathrm{~m}, 5 \mathrm{H}), 5.14(\mathrm{~s}, 2 \mathrm{H}), 2.38\left(\mathrm{t},{ }^{3} \mathrm{~J}_{H H}=7.5 \mathrm{~Hz}\right.$, $2 \mathrm{H}), 1.72-1.63(\mathrm{~m}, 2 \mathrm{H}), 1.35-1.31(\mathrm{~m}, 4 \mathrm{H}), 0.91\left(\mathrm{t},{ }^{3} \mathrm{~J}_{H H}=6.4 \mathrm{~Hz}, 3 \mathrm{H}\right) .{ }^{13} \mathrm{C}$ NMR (150 MHz, $\left.\mathrm{CDCl}_{3}\right): \delta[\mathrm{ppm}]=173.61,136.21,128.50,128.12,66.01,34.3,31.29,24.63,22.26,13.83$. IR (ATR): $\tilde{v}\left[\mathrm{~cm}^{-1}\right]=2957,2930,2872,1735,1456,1379,1352,1344,1243,1213,1162$, 973, 733, 696. HR-MS (El) $\mathrm{m} / z$ calcd for $\left[\mathrm{C}_{13} \mathrm{H}_{18} \mathrm{O}_{2}\right]^{+}[\mathrm{M}]^{+} 206.1307$ found 206.131. 


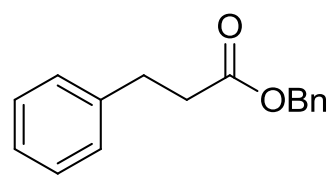

\section{Benzyl 3-phenylpropanoate (26s)}

${ }^{1} \mathbf{H}$ NMR (300 MHz, $\left.\mathbf{C D C l}_{3}\right): \delta[\mathrm{ppm}]=7.40-7.30(\mathrm{~m}, 7 \mathrm{H}), 7.25-7.22(\mathrm{~m}, 3 \mathrm{H}), 5.15(\mathrm{~s}, 2 \mathrm{H})$, $3.01\left(\mathrm{t},{ }^{3} \mathrm{~J}_{H H}=7.6 \mathrm{~Hz}\right), 2.72\left(\mathrm{t},{ }^{3} \mathrm{~J}_{H H}=7.6 \mathrm{~Hz}\right) .{ }^{13} \mathrm{C} \mathrm{NMR}\left(75 \mathrm{MHz}, \mathbf{C D C l}_{3}\right): \delta[\mathrm{ppm}]=172.75$, 140.43, 136.0, 128.60, 128.53, 128.33, 128.24, 126.30, 66.31, 35.92, 30.97. IR (ATR): $\tilde{v}$ $\left[\mathrm{cm}^{-1}\right]=3030,2953,1732,1496,1454,1381,1147,1078,1028,906,746,694$. HR-MS (EI) $\mathrm{m} / \mathrm{z}$ calcd for $\left[\mathrm{C}_{16} \mathrm{H}_{16} \mathrm{O}_{2}\right][\mathrm{M}] 240.1150$ found 240.116 .<smiles>O=C(CCc1ccc(Cl)cc1)OCc1ccccc1</smiles>

\section{Benzyl 3-(4-chlorophenyl)propanoate (26t)}

${ }^{1} \mathrm{H}$ NMR (300 MHz, $\left.\mathrm{CDCl}_{3}\right): \delta[\mathrm{ppm}]=7.40-7.25(\mathrm{~m}, 7 \mathrm{H}), 7.15-7.12(\mathrm{~m}, 2 \mathrm{H}), 5.14(\mathrm{~s}, 2 \mathrm{H})$, $2.97\left(\mathrm{t},{ }^{3} \mathrm{~J}_{H H}=7.5 \mathrm{~Hz}, 2 \mathrm{H}\right), 2.69\left(\mathrm{t},{ }^{3} \mathrm{~J}_{H H}=7.5 \mathrm{~Hz}, 2 \mathrm{H}\right) .{ }^{13} \mathrm{C}$ NMR (75 MHz, $\left.\mathbf{C D C l}_{3}\right): \delta[\mathrm{ppm}]=$ 172.34, 138.84, 132.09, 129.69, 128.60, 128.55, 128.25, 128.22, 66.34, 35.70, 30.29. IR (ATR): $\tilde{v}\left[\mathrm{~cm}^{-1}\right]=2960,2929,1732,1608,1490,1454,1381,1255,1149,1091,1014,819$, 750, 734, 696. HR-MS (EI) $m / z$ calcd for $\left[\mathrm{C}_{16} \mathrm{H}_{15} \mathrm{ClO}_{2}\right]$ [M] 274.0760 found 274.076.<smiles>COc1ccc(CCC(=O)OCc2ccccc2)cc1</smiles>

\section{Benzyl 3-(4-methoxyphenyl)propanoate (26u)}

${ }^{1} \mathbf{H}$ NMR (600 MHz, $\left.\mathbf{C D C l}_{3}\right): \delta[p p m]=7.39-7.32(\mathrm{~m}, 5 \mathrm{H}), 7.15-7.12(\mathrm{~m}, 2 \mathrm{H}), 6.86-6.83(\mathrm{~m}$, 2H), $5.15(\mathrm{~s}, 2 \mathrm{H}), 3.81(\mathrm{~s}, 3 \mathrm{H}), 2.96\left(\mathrm{t},{ }^{3} \mathrm{~J}_{H H}=7.5 \mathrm{~Hz}\right), 2.69\left(\mathrm{t},{ }^{3} \mathrm{~J}_{H H}=7.5 \mathrm{~Hz}\right) .{ }^{13} \mathrm{C}$ NMR (150 $\left.\mathbf{M H z}, \mathbf{C D C l}_{3}\right): \delta[\mathrm{ppm}]=172.73,158.16,136.04,132.51,129.25,128.52,128.18,128.17$, 113.96, 66.20, 55.23, 36.20, 30.14. IR (ATR): $\tilde{v}\left[\mathrm{~cm}^{-1}\right]=2953,2931,1732,1610,1512$, 1454, 1300, 1244, 1176, 1107, 1147, 1033, 825, 734, 696. HR-MS (El) m/z calcd for $\left[\mathrm{C}_{17} \mathrm{H}_{18} \mathrm{O}_{3}\right][\mathrm{M}] 270.1256$ found 270.126 .<smiles>C/C=C/CCC(=O)OCc1ccccc1</smiles>

Benzyl (4E)-hex-4-enoate (26v)

${ }^{1} \mathbf{H}$ NMR (600 MHz, $\left.\mathbf{C D C l}_{3}\right): \delta[p p m]=7.41-7.33(\mathrm{~m}, 5 \mathrm{H}), 5.53-5.42(\mathrm{~m}, 2 \mathrm{H}), 5.15(\mathrm{~s}, 2 \mathrm{H})$, 2.46-2.43 (m, 2H), 2.37-2.33 (m 2H), 1.67-1.64 (m, 3H). $\left.{ }^{13} \mathbf{C ~ N M R ~ ( 1 5 0 ~ M H z , ~} \mathbf{C D C l}_{3}\right): \delta$ [ppm] 
$=173.10,136.11,129.10,128.55,128.21,128.19,126.29,66.14,34.33,27.91,17.89 . \mathbf{I R}$ (ATR): $\tilde{v}\left[\mathrm{~cm}^{-1}\right]=2933,1734,1454,1379,1257,1157,966,910,736,696$. HR-MS (EI) $\mathrm{m} / \mathrm{z}$ calcd for $\left[\mathrm{C}_{13} \mathrm{H}_{16} \mathrm{O}_{2}\right]^{+}[\mathrm{M}]^{+} 204.1150$ found 204.114 .<smiles>CCOC(=O)C(CC(=O)OCc1ccccc1)c1ccccc1</smiles>

\section{4-(benzyl) 1-ethyl-2-phenylsuccinate(26q)}

${ }^{1} \mathrm{H}$ NMR (600 MHz, $\left.\mathbf{C D C l}_{3}\right): \delta[p p m]=7.39-7.30(\mathrm{~m}, 10 \mathrm{H}), 5.15 .\left(\mathrm{q}, 2 \mathrm{H},{ }^{3} \mathrm{JHH}_{\mathrm{HH}}=7.4 \mathrm{~Hz}\right), 4.2-$ $4.1(\mathrm{~m}, 3 \mathrm{H}), 3.3\left(\mathrm{dd},{ }^{3} \mathrm{~J}_{\mathrm{HH}}=10 \mathrm{~Hz},{ }^{2} J_{\mathrm{HH}}=16.9 \mathrm{~Hz}\right), 2.77\left(\mathrm{dd},{ }^{3} J_{\mathrm{HH}}=5.46 \mathrm{~Hz},{ }^{2} J_{\mathrm{HH}}=16.9 \mathrm{~Hz}\right)$, $1.20\left(\mathrm{t},{ }^{3} \mathrm{~J}_{\mathrm{HH}}=7.4 \mathrm{~Hz}\right) .{ }^{13} \mathrm{C}$ NMR $\left(150 \mathrm{MHz}, \mathbf{C D C l}_{3}\right): \delta[\mathrm{ppm}]=172.9,171.3,137.8,135.7$, $128.8,128.6,128.3,128.2,127.8,127.6,66.5,61.2,47.3,37.9,14.04$.

10 Procedure for the one step synthesis of nepetalactone<smiles>CC(C=O)=CCCC(C)=CC=O</smiles>
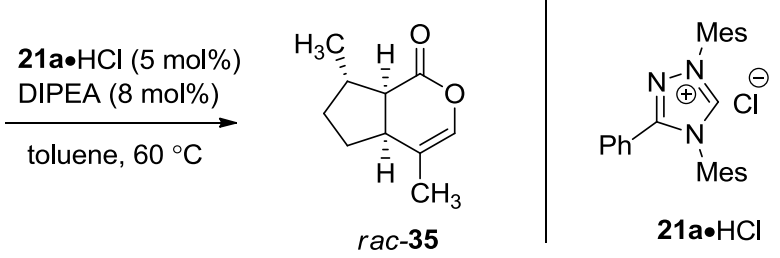

In a glove box, a screw-capped GC vial was charged with the triazolium salt $21 \mathrm{a} \cdot \mathrm{HCl}(0.05$ equiv), 10 -oxo citral $34^{[24]}$ (100 $\mathrm{mg}, 1.0$ equiv), DIPEA (0.08 equiv) and toluene $(0.2 \mathrm{ml})$. The reaction mixture was stirred at $60{ }^{\circ} \mathrm{C}$ for $6 \mathrm{~h}$ (full aldehyde consumption according to GCanalysis). The reaction mixture was cooled to room temperature, and the product nepetalactone (rac-35) was purified by flash column chromatography on silica gel.

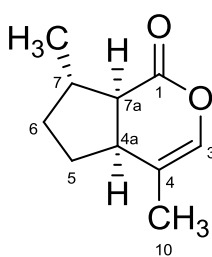

${ }^{1} \mathrm{H}$ NMR (600 MHz, $\left.\mathrm{CDCl}_{3}\right): \delta[\mathrm{ppm}]=[\mathrm{ppm}]:$ 6.18-6.17 $(\mathrm{m}, 1 \mathrm{H}, \mathrm{H} 3), 2.78-$ $2.74(\mathrm{~m}, 1 \mathrm{H}, \mathrm{H} 4 \mathrm{a}), 2.47-2.44(\mathrm{~m}, 1 \mathrm{H}, \mathrm{H} 7 \mathrm{a}), 2.40-2.35(\mathrm{~m}, 1 \mathrm{H}, \mathrm{H} 7)$, 2.07$2.02(\mathrm{~m}, 1 \mathrm{H}, \mathrm{H} 5 \mathrm{a}), 1.93-1.88(\mathrm{~m}, 1 \mathrm{H}, \mathrm{H} 6 \mathrm{a}), 1.64-1.63(\mathrm{~m}, 3 \mathrm{H}, \mathrm{H} 10)$, 1.59-1.53 (m, 1H, H5b), 1.27-1.22 (m, 1H, H6b), $1.20\left(\mathrm{~d}, 3 \mathrm{H},{ }^{3} J_{\mathrm{HH}}=6.6\right.$

$\mathrm{Hz}, \mathrm{H} 9) .{ }^{13} \mathrm{C}$ NMR (75 MHz, $\left.\mathrm{CDCl}_{3}\right): \delta[\mathrm{ppm}]=170.92$ (1C, C1), 133.6 (1C, C2), 115.34 (1C, C3), 49.38 (1C, C8), 40.73 (1C, C9), 39.75 (1C, C7), 33.03 (1C, C6), 30.92 (1C, C5), 20.26 (1C, C4a), 15.48 (1C, C7a). 


\section{NMR spectra}

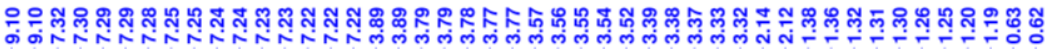

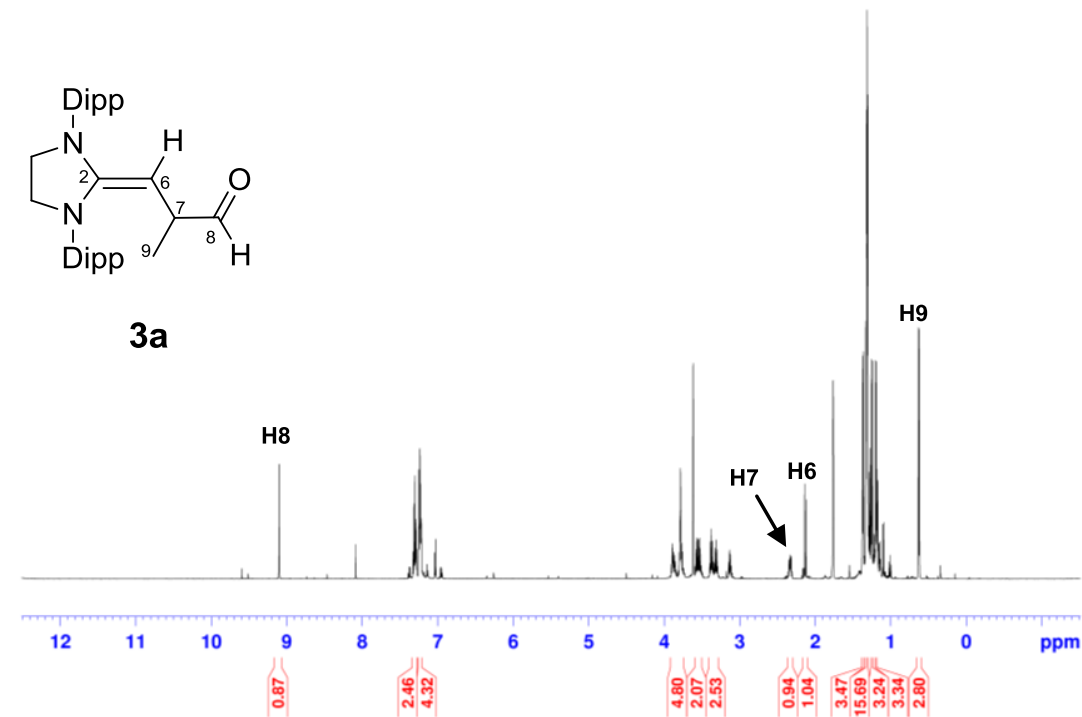

Figure $S 1^{1} \mathrm{H}(600 \mathrm{MHz})$ NMR spectrum of $3 \mathbf{a}\left(\mathrm{THF}-d_{8}, 298 \mathrm{~K}\right)$.

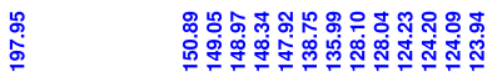

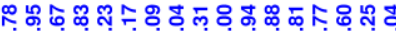

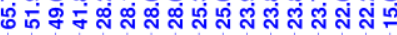

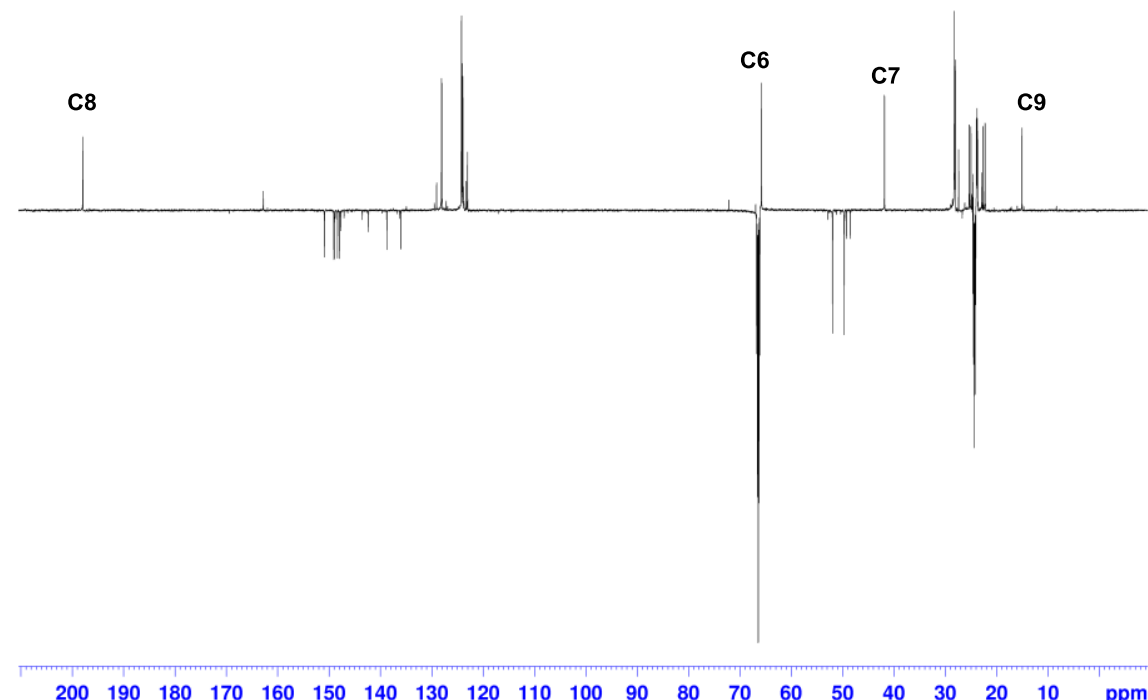

Figure $\mathbf{S 2}{ }^{13} \mathrm{C}(150 \mathrm{MHz})$ DEPTQ NMR spectrum of $\mathbf{3 a}\left(\mathrm{THF}-\mathrm{d}_{8}, 298 \mathrm{~K}\right)$. 


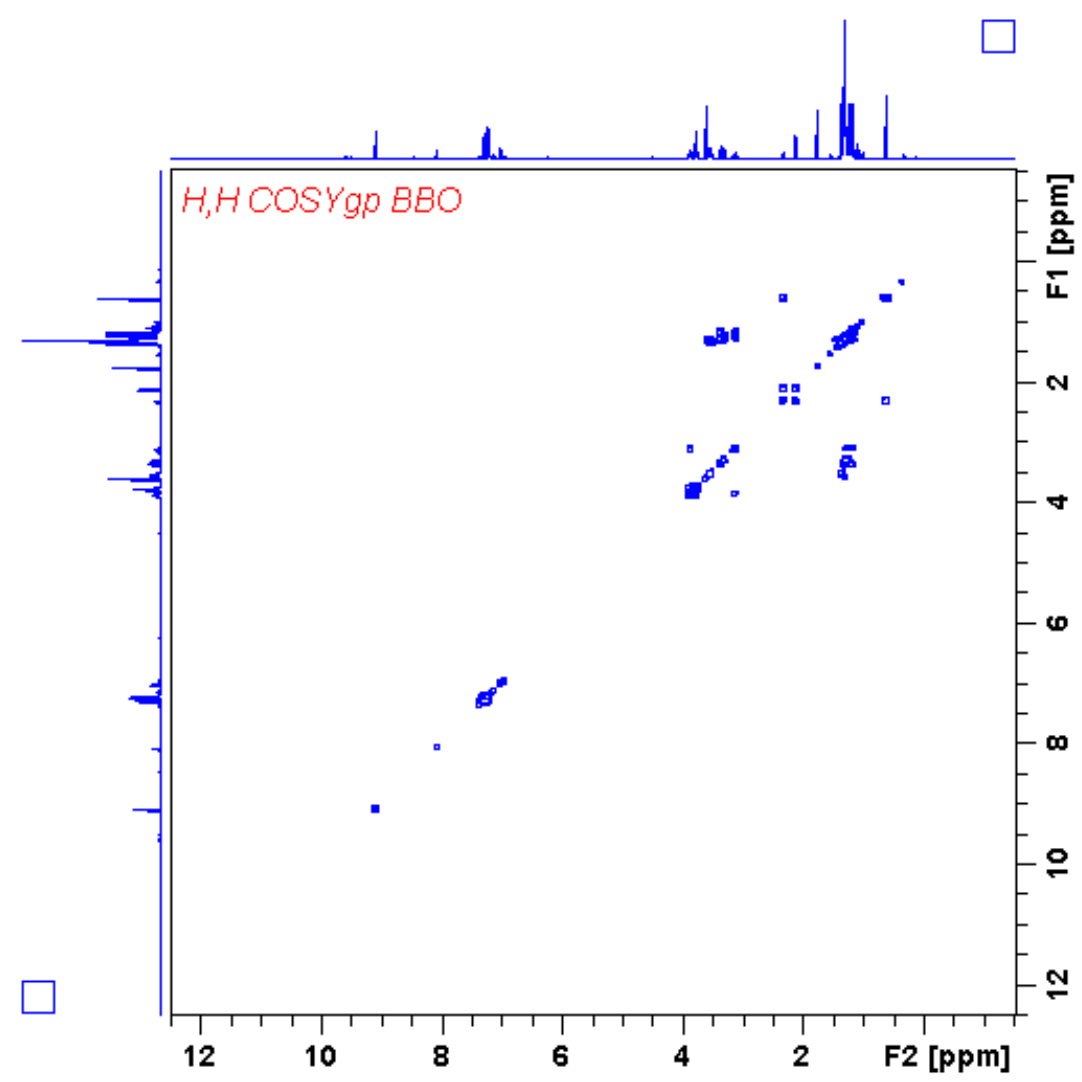

Figure $S 3{ }^{1} \mathrm{H},{ }^{1} \mathrm{H}$ COSY $(600 \mathrm{MHz})$ NMR spectrum of $\mathbf{3 a}\left(\mathrm{THF}-\mathrm{d}_{8}, 298 \mathrm{~K}\right)$.

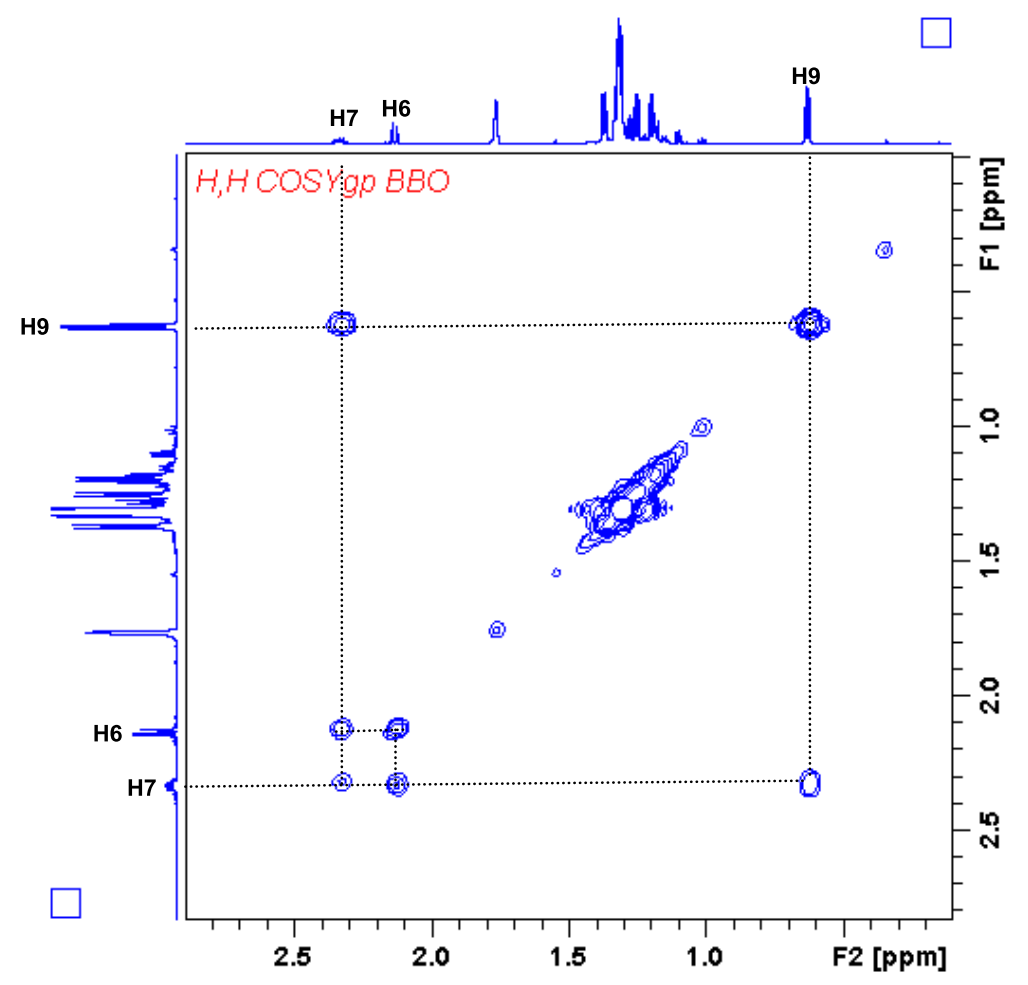

Figure S4 Part of ${ }^{1} \mathrm{H},{ }^{1} \mathrm{H}$ COSY $(600 \mathrm{MHz})$ NMR spectrum of $3 \mathbf{3}\left(\mathrm{THF}-d_{8}, 298 \mathrm{~K}\right)$. 


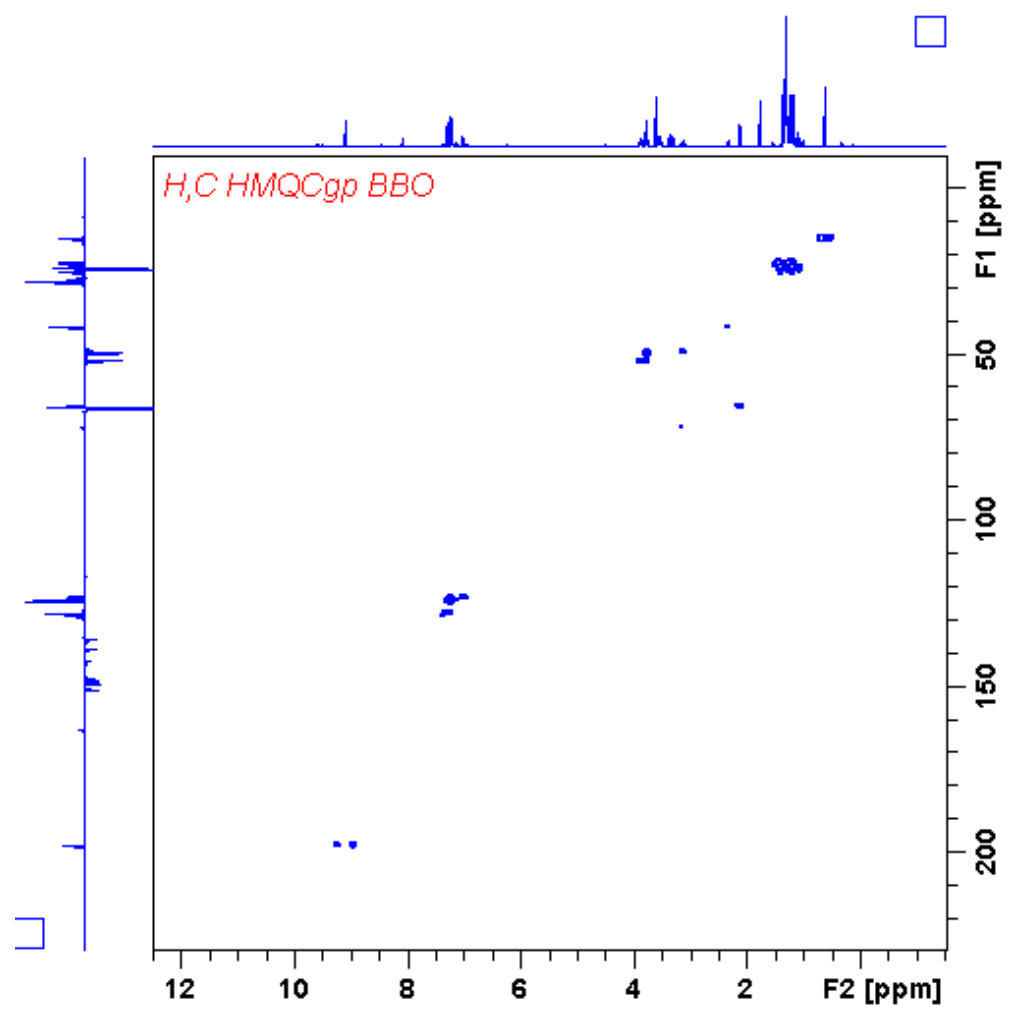

Figure $S 5{ }^{1} \mathrm{H}(600 \mathrm{MHz}),{ }^{13} \mathrm{C}(150 \mathrm{MHz}) \mathrm{HMQC}$ NMR spectrum of $3 \mathbf{a}\left(\mathrm{THF}-\mathrm{d}_{8}, 298 \mathrm{~K}\right)$.

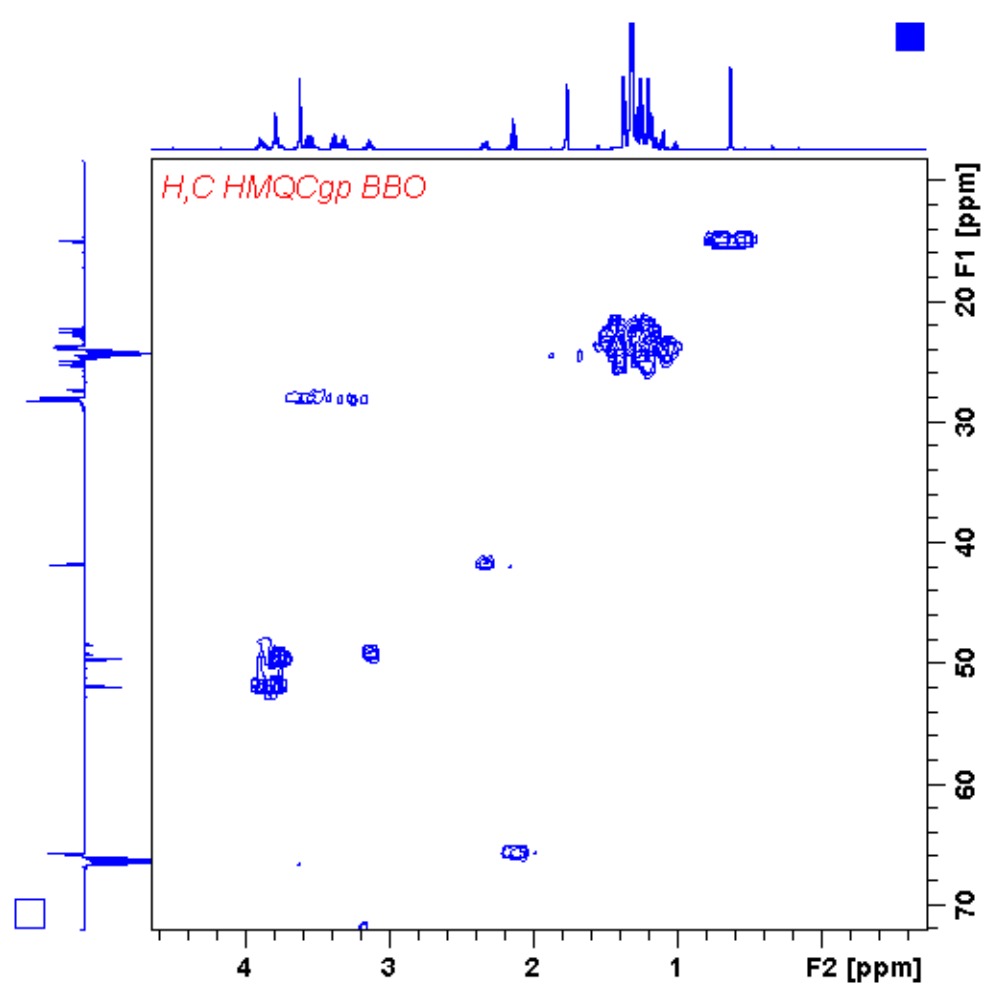

Figure S6 Part of ${ }^{1} \mathrm{H}(600 \mathrm{MHz}),{ }^{13} \mathrm{C}(150 \mathrm{MHz}) \mathrm{HMQC}$ NMR spectrum of $3 \mathbf{a}\left(\mathrm{THF}-d_{8}, 298 \mathrm{~K}\right)$. 


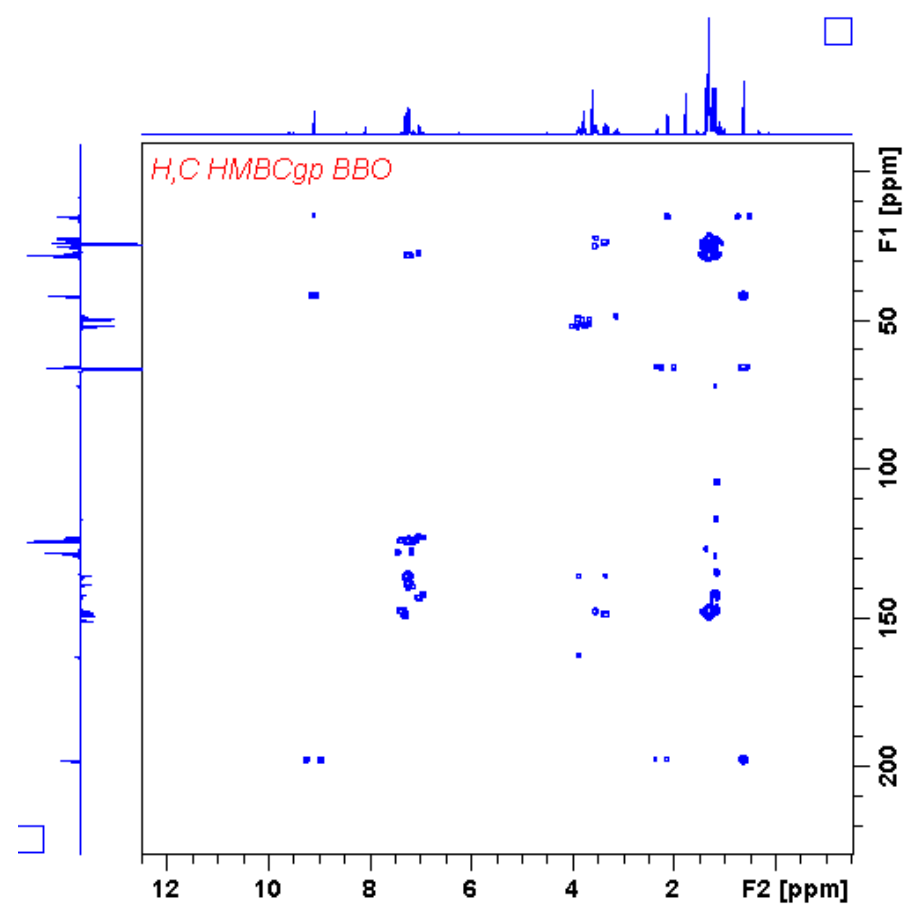

Figure $S 7^{1} \mathrm{H}(600 \mathrm{MHz}),{ }^{13} \mathrm{C}(150 \mathrm{MHz}) \mathrm{HMBC}$ NMR spectrum of $3 \mathbf{a}\left(\mathrm{THF}-\mathrm{d}_{8}, 298 \mathrm{~K}\right)$.

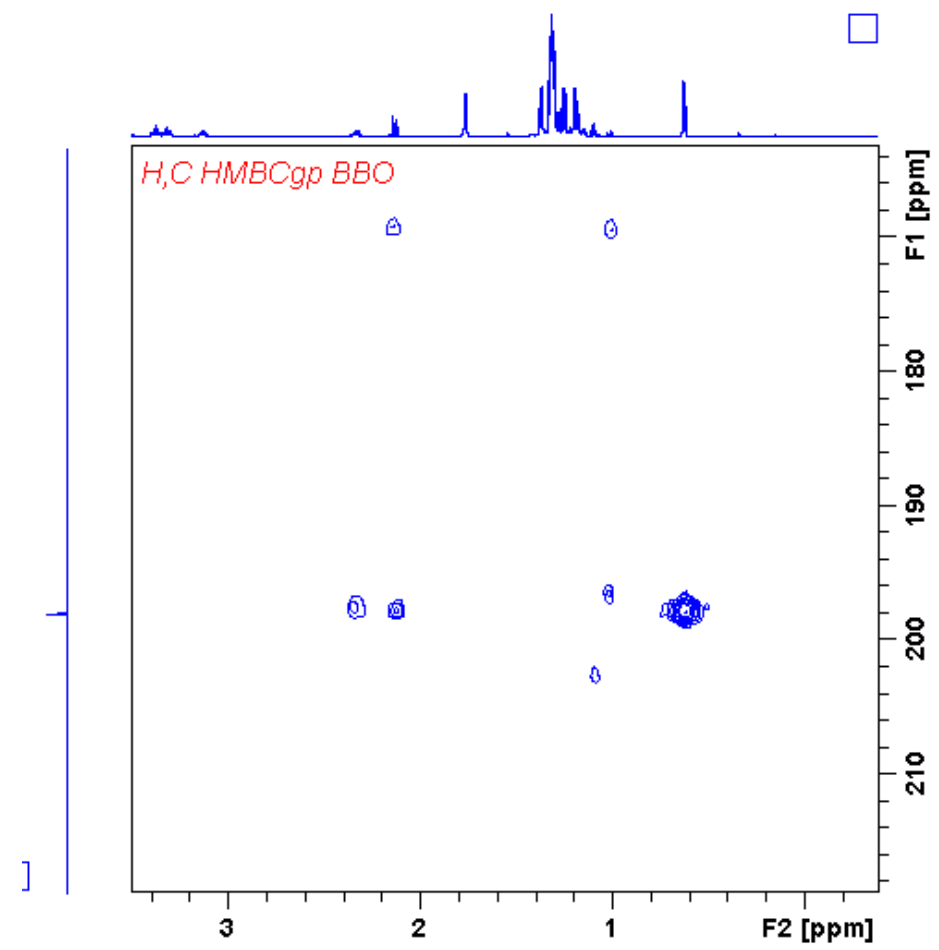

Figure S8 Part of ${ }^{1} \mathrm{H}(600 \mathrm{MHz}),{ }^{13} \mathrm{C}(150 \mathrm{MHz}) \mathrm{HMBC}$ NMR spectrum of $3 \mathbf{a}\left(\mathrm{THF}-d_{8}, 298 \mathrm{~K}\right)$. 


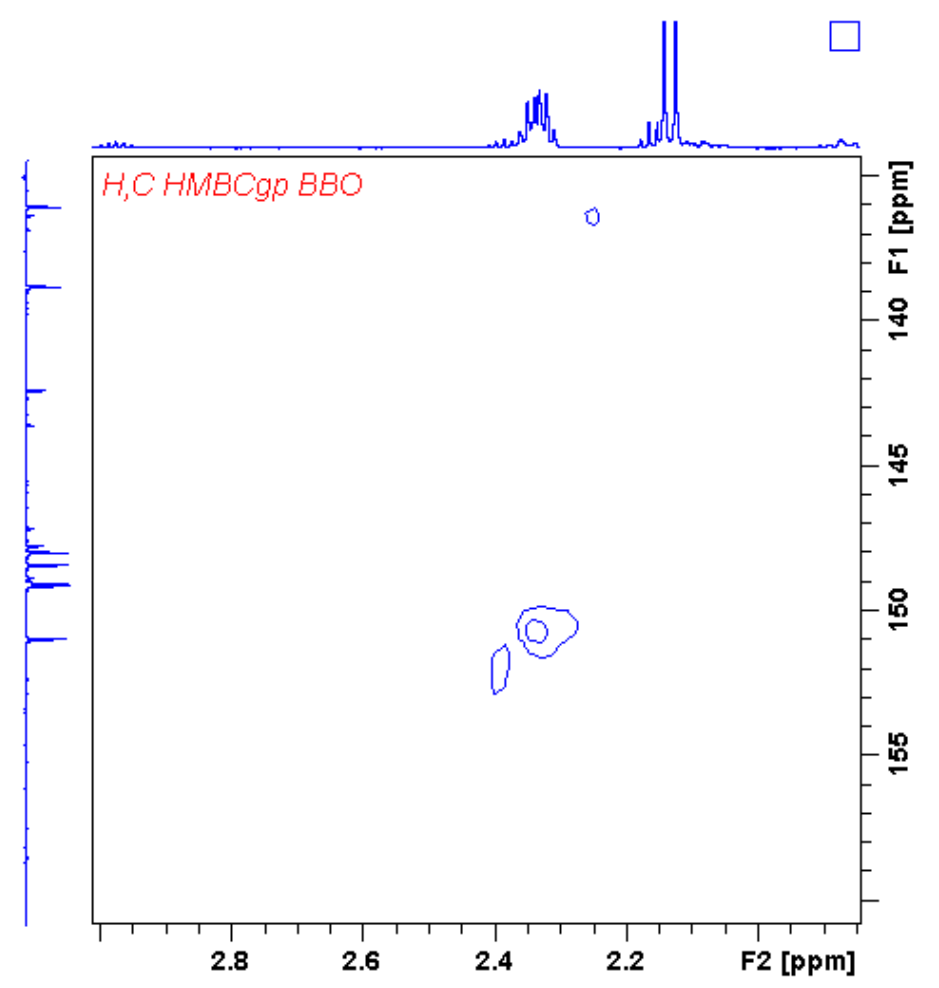

Figure S9 Part of ${ }^{1} \mathrm{H}(600 \mathrm{MHz}),{ }^{13} \mathrm{C}(150 \mathrm{MHz}) \mathrm{HMBC}$ NMR spectrum of $\mathbf{3 a}\left(\mathrm{THF}-d_{8}, 298 \mathrm{~K}\right)$.

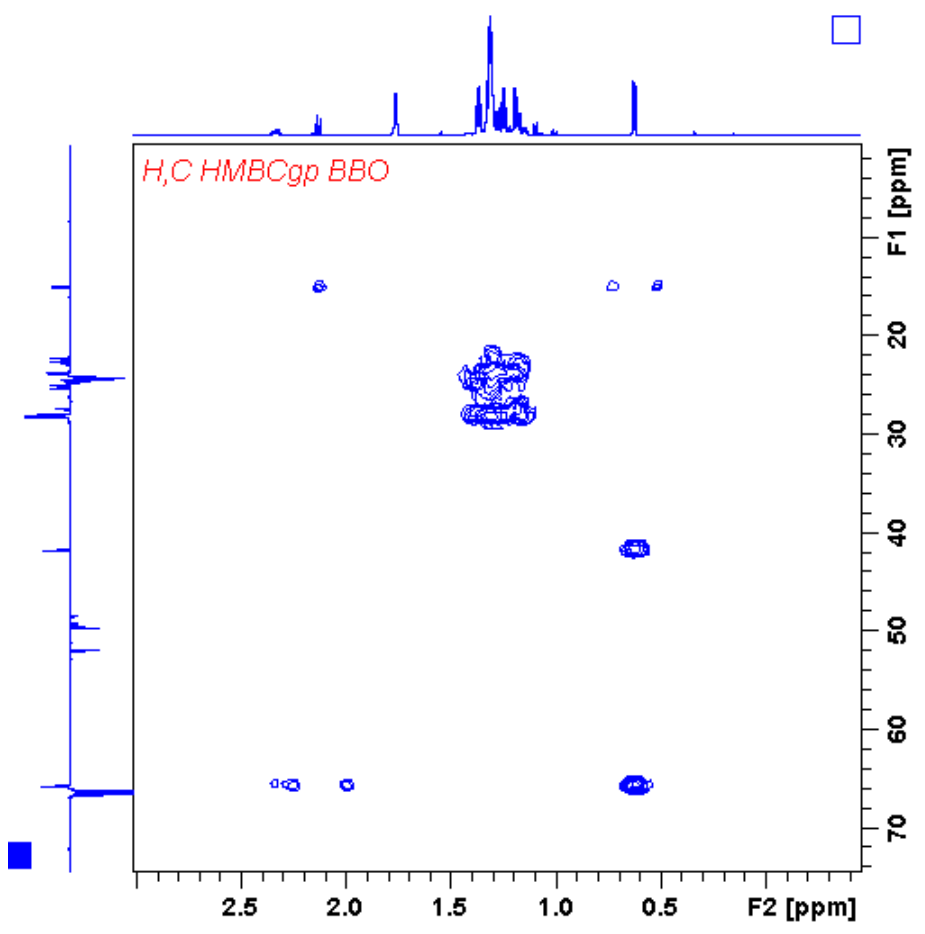

Figure S10 Part of ${ }^{1} \mathrm{H}(600 \mathrm{MHz}),{ }^{13} \mathrm{C}(150 \mathrm{MHz}) \mathrm{HMBC}$ NMR spectrum of $3 \mathbf{a}\left(\mathrm{THF}-d_{8}, 298 \mathrm{~K}\right)$. 


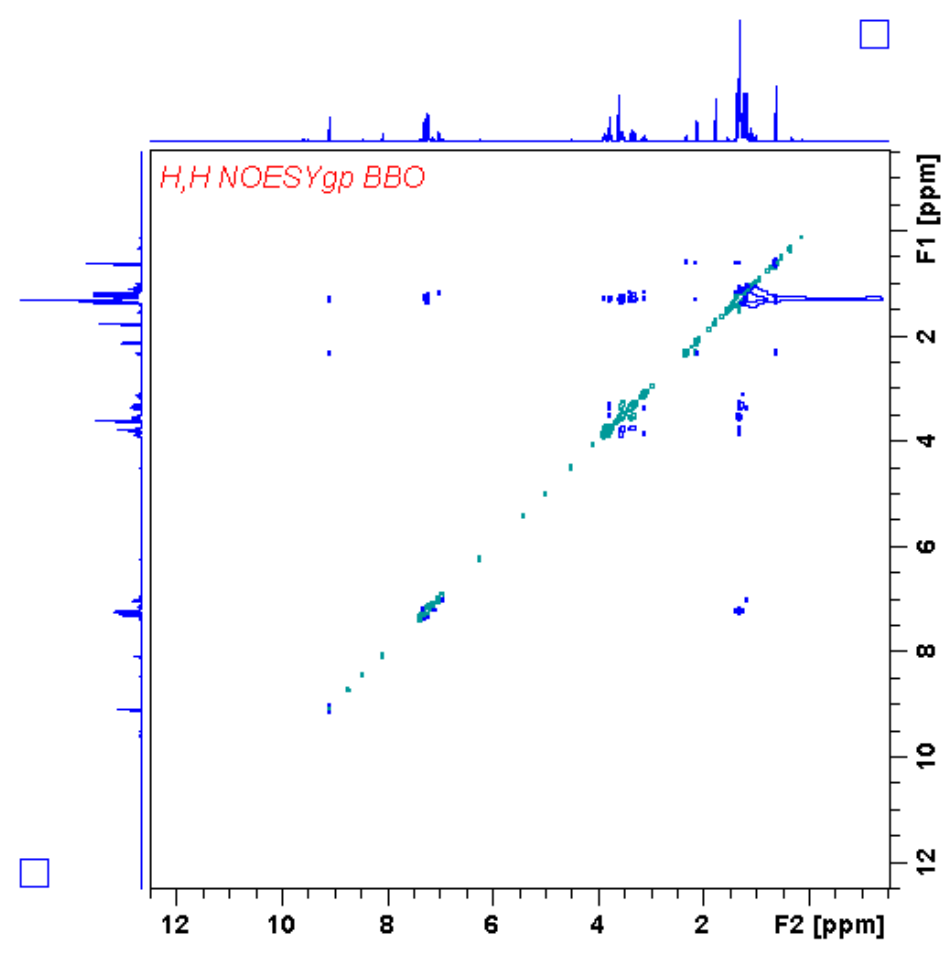

Figure $S 11{ }^{1} \mathrm{H},{ }^{1} \mathrm{H}$ NOESY NMR spectrum of $3 \mathbf{3 a}\left(\mathrm{THF}-\mathrm{d}_{8}, 600 \mathrm{MHz}, 298 \mathrm{~K}\right.$, mixing time = $600 \mathrm{~ms}$ ). 


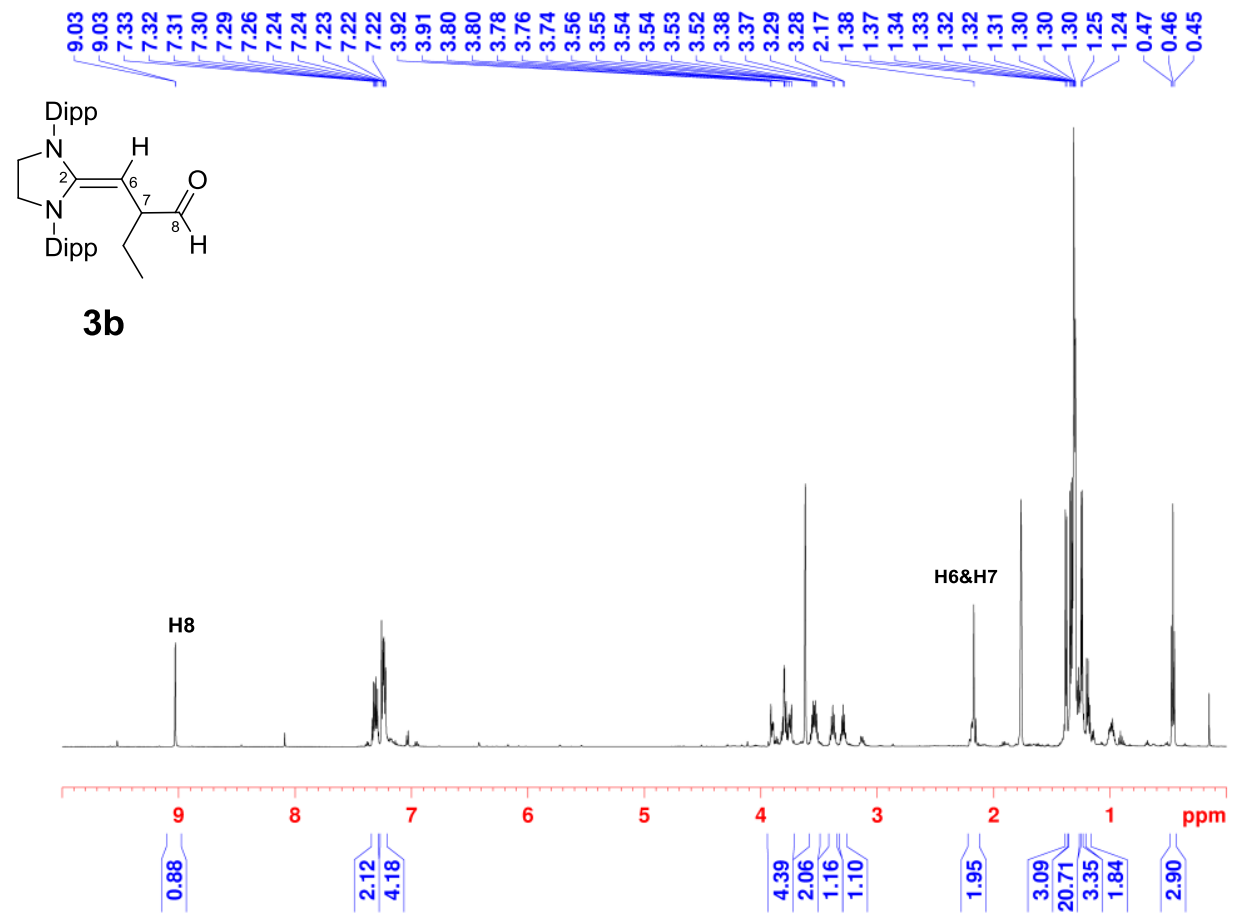

Figure $\mathbf{S 1 2}{ }^{1} \mathrm{H}(600 \mathrm{MHz}) \mathrm{NMR}$ spectrum of $\mathbf{3 b}\left(\mathrm{THF}-d_{8}, 298 \mathrm{~K}\right)$.
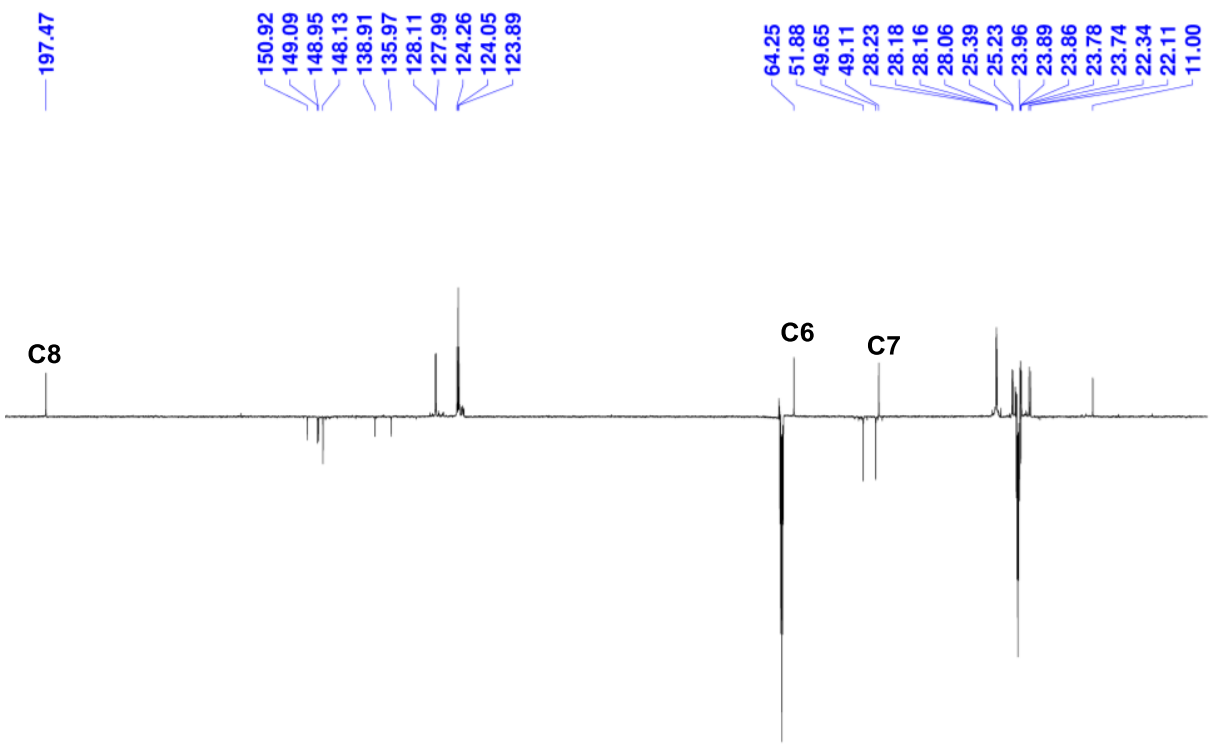

$\begin{array}{lllllllllllllllllllll}200 & 190 & 180 & 170 & 160 & 150 & 140 & 130 & 120 & 110 & 100 & 90 & 80 & 70 & 60 & 50 & 40 & 30 & 20 & 10 & \mathrm{ppm}\end{array}$

Figure $\mathbf{S 1 3}{ }^{13} \mathrm{C}(150 \mathrm{MHz})$ DEPTQ NMR spectrum of $\mathbf{3 b}\left(\mathrm{THF}-d_{8}, 298 \mathrm{~K}\right)$. 


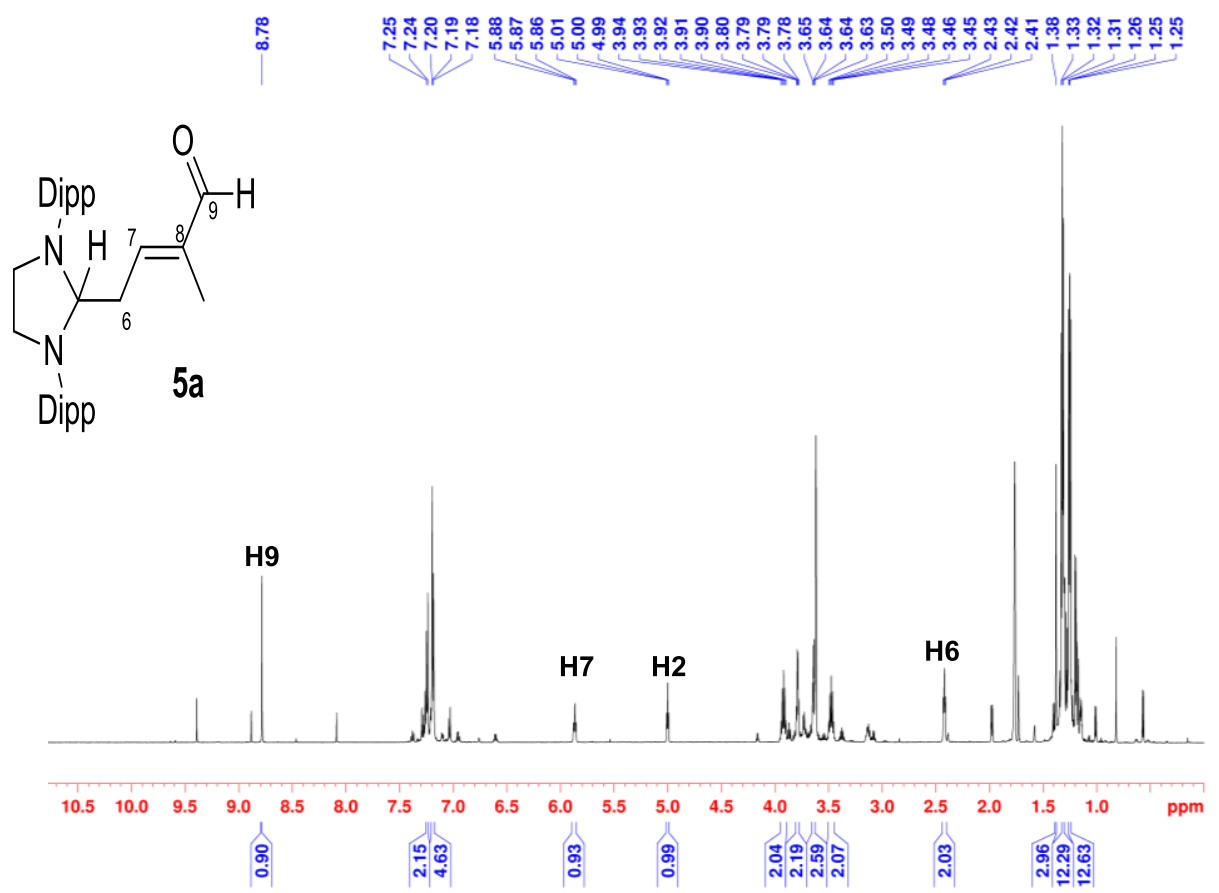

Figure $S 14{ }^{1} \mathrm{H}(600 \mathrm{MHz}) \mathrm{NMR}$ spectrum of $5 \mathbf{a}\left(\mathrm{THF}-d_{8}, 298 \mathrm{~K}\right)$.

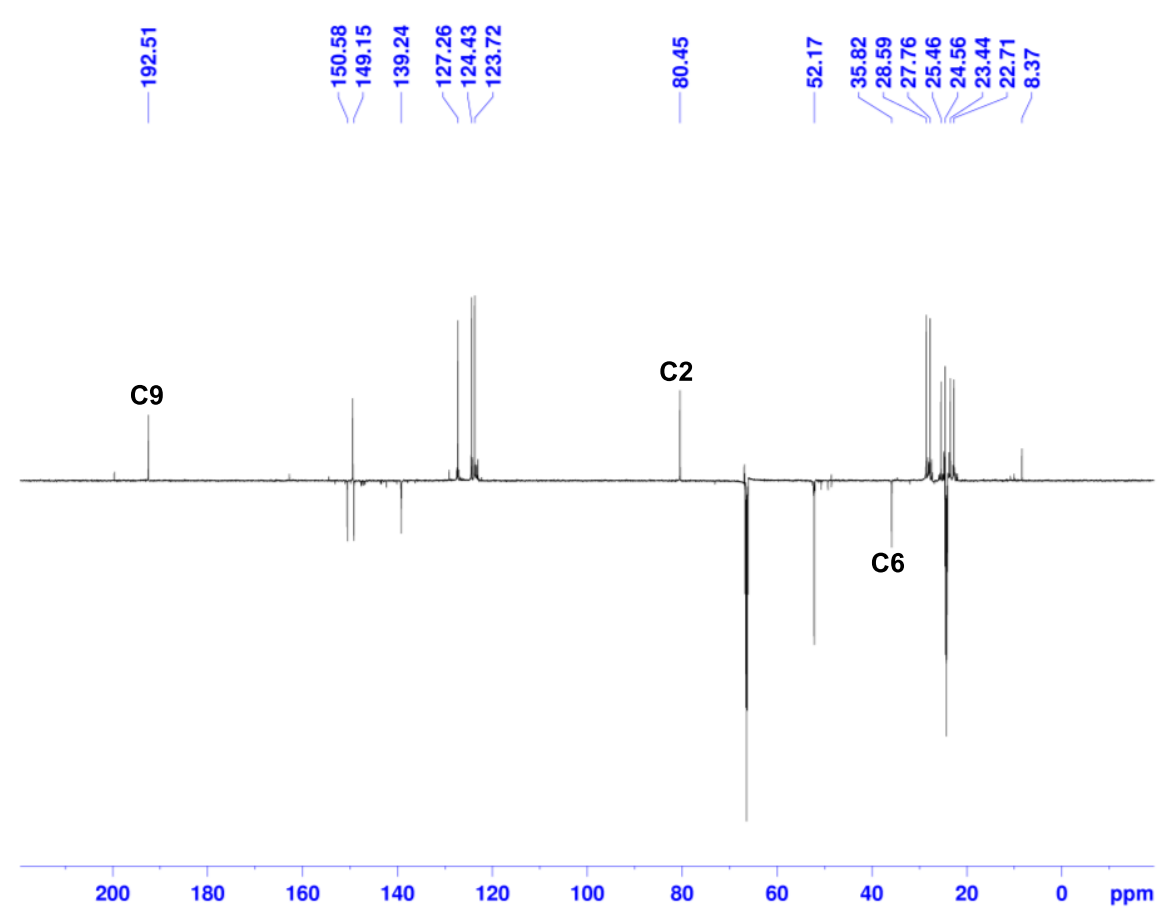

Figure $S 15{ }^{13} \mathrm{C}(150 \mathrm{MHz})$ DEPTQ NMR spectrum of $5 \mathbf{a}\left(\mathrm{THF}-d_{8}, 298 \mathrm{~K}\right)$. 


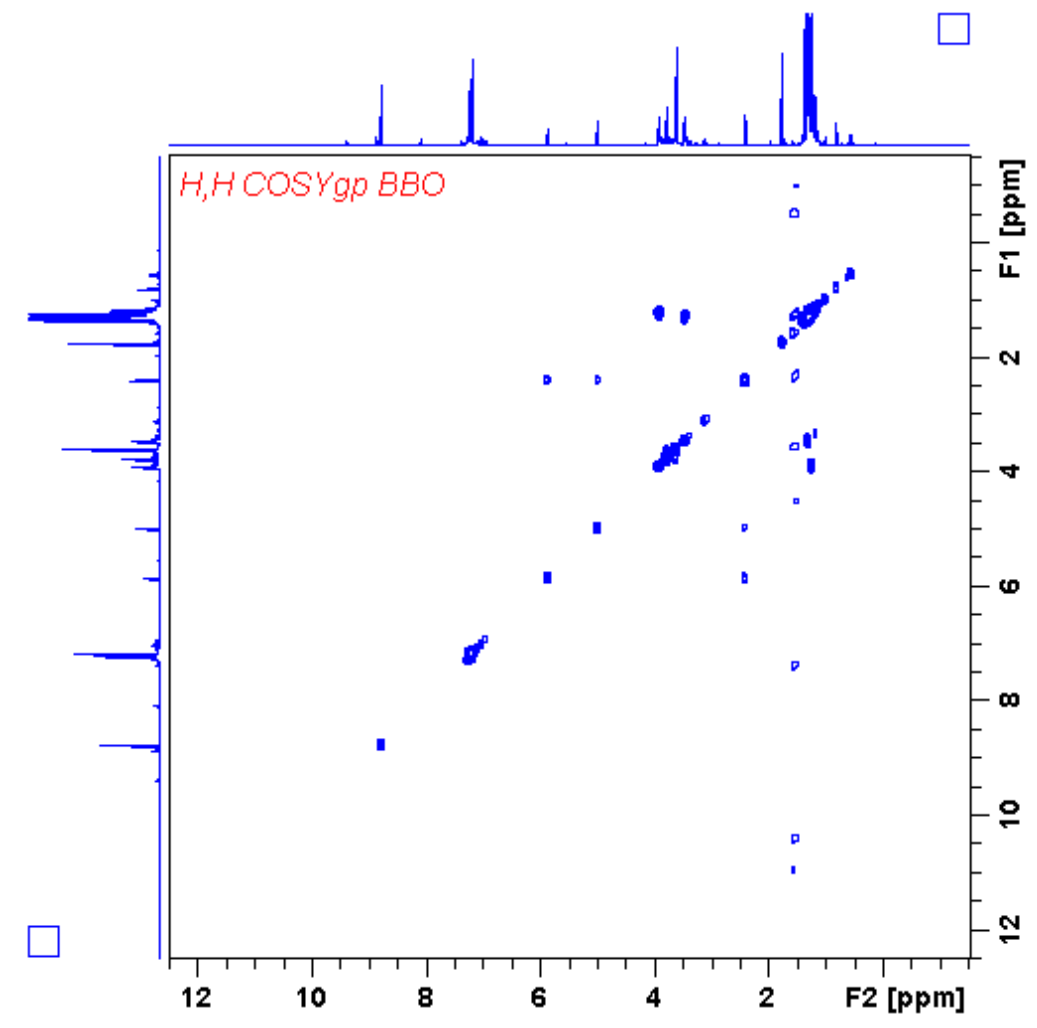

Figure $S 16{ }^{1} \mathrm{H},{ }^{1} \mathrm{H} \operatorname{COSY}(600 \mathrm{MHz})$ NMR spectrum of $5 \mathrm{a}\left(\mathrm{THF}-d_{8}, 298 \mathrm{~K}\right)$.

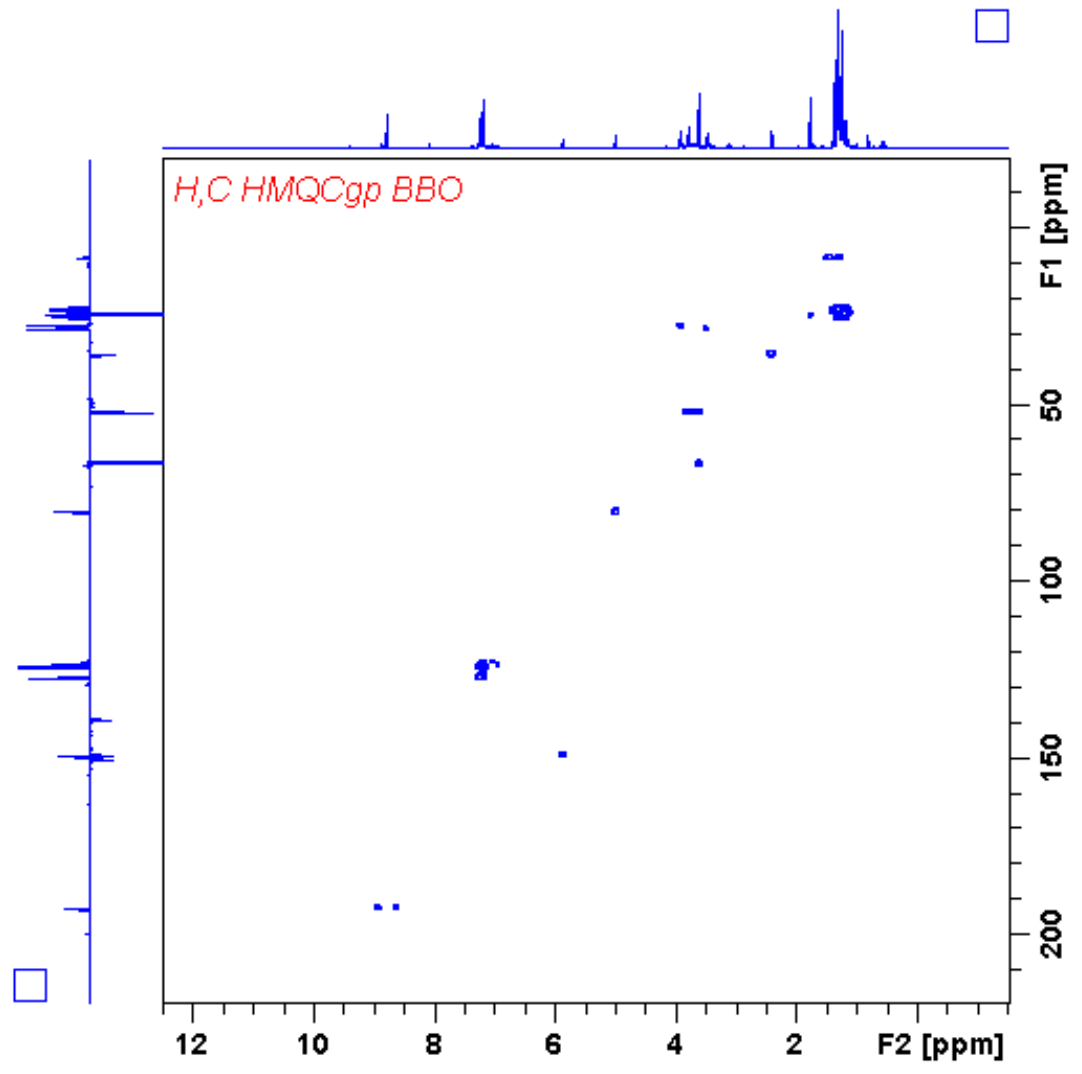

Figure $S 17^{1} \mathrm{H}(600 \mathrm{MHz}),{ }^{13} \mathrm{C}(150 \mathrm{MHz}) \mathrm{HMQC}$ NMR spectrum of $\mathbf{5 a}\left(\mathrm{THF}-d_{8}, 298 \mathrm{~K}\right)$. 


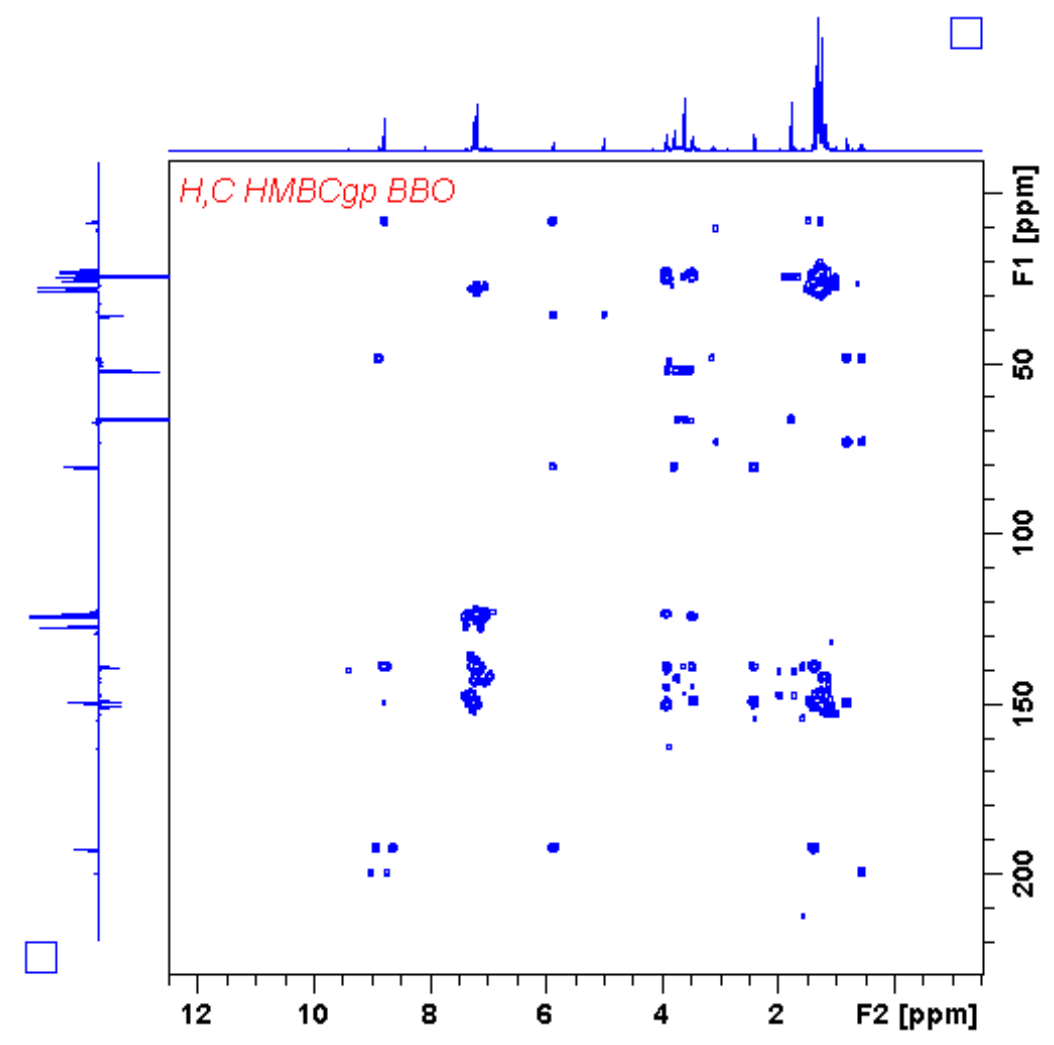

Figure $\mathbf{S 1 8}{ }^{1} \mathrm{H}(600 \mathrm{MHz}),{ }^{13} \mathrm{C}(150 \mathrm{MHz}) \mathrm{HMBC}$ NMR spectrum of $\mathbf{5 a}\left(\mathrm{THF}-\mathrm{d}_{8}, 298 \mathrm{~K}\right)$.

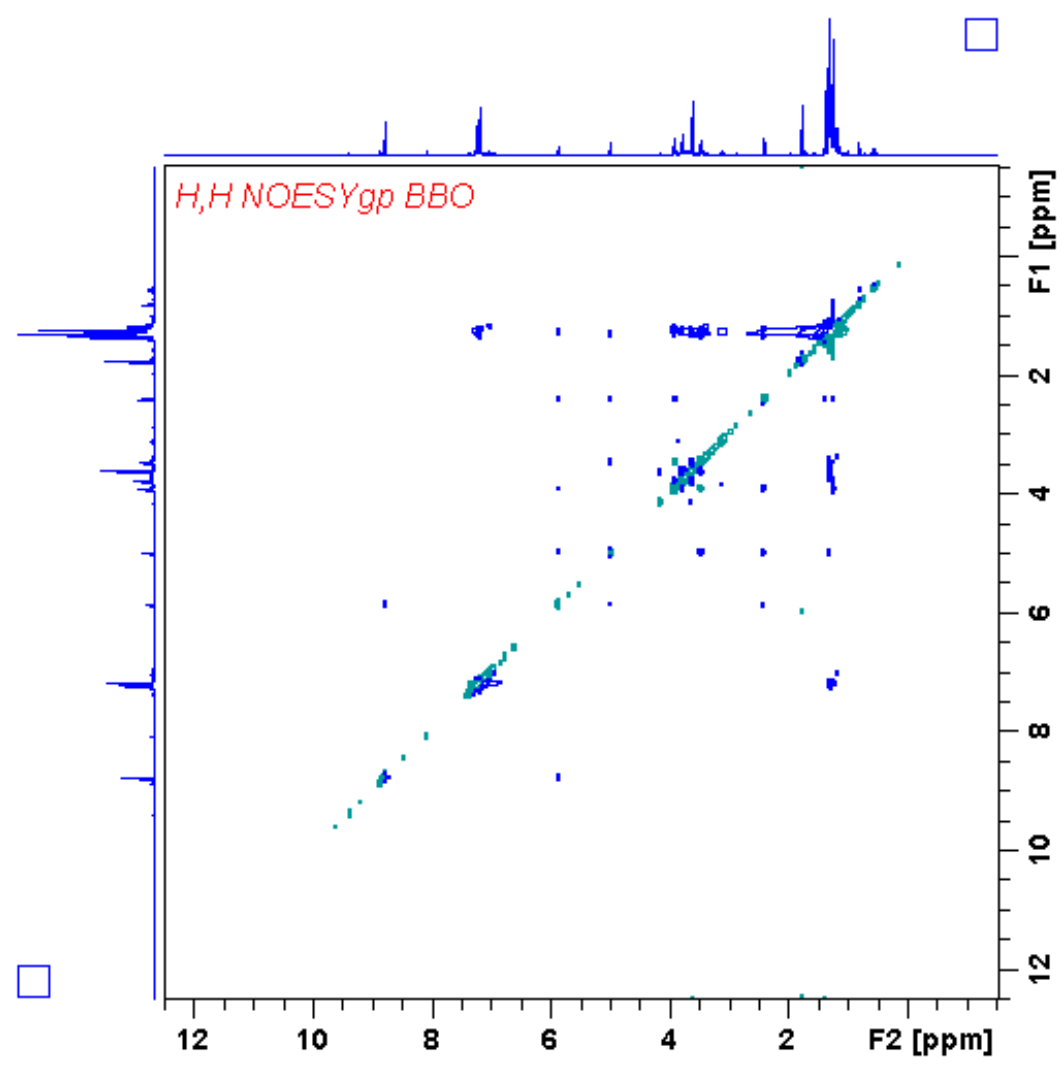

Figure $S 19{ }^{1} \mathrm{H},{ }^{1} \mathrm{H}$ NOESY NMR spectrum of $5 \mathbf{a}\left(\mathrm{THF}^{-} \mathrm{d}_{8}, 600 \mathrm{MHz}, 298 \mathrm{~K}\right.$, mixing time $=$ $600 \mathrm{~ms})$. 

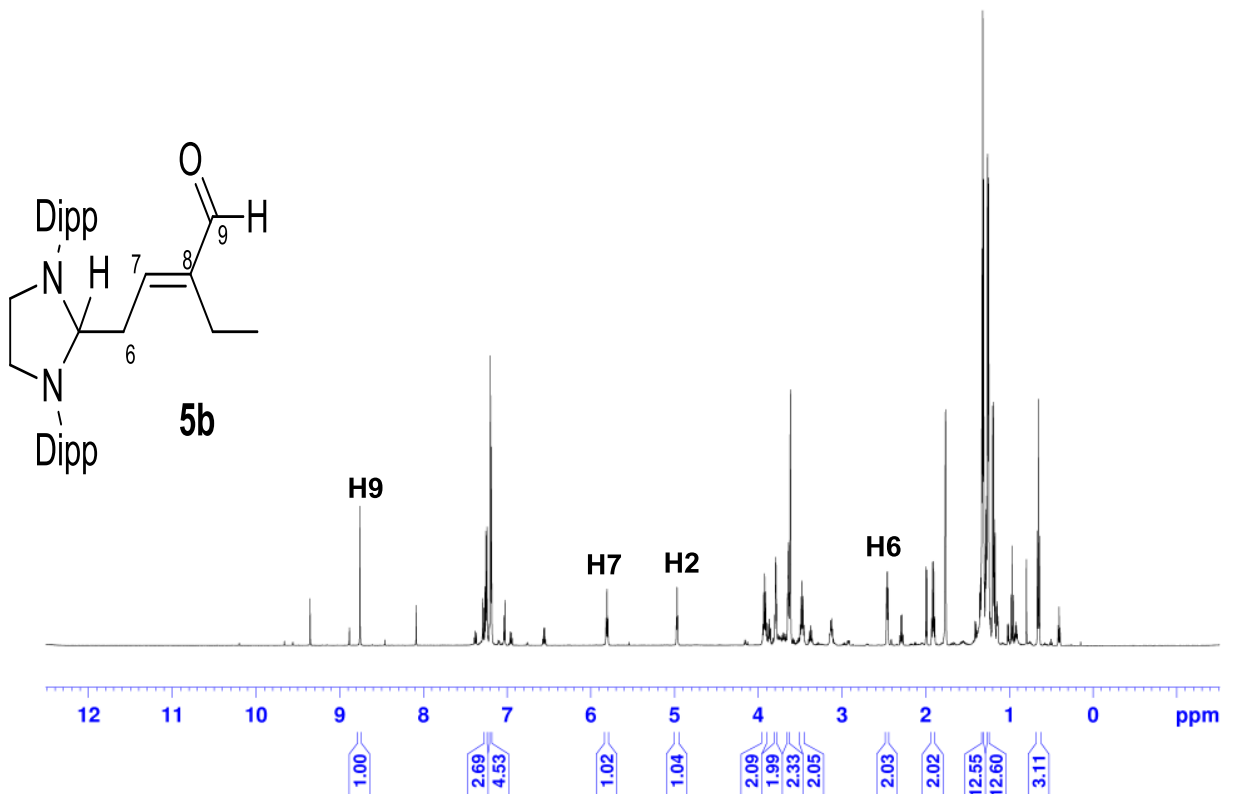

Figure $520{ }^{1} \mathrm{H}(600 \mathrm{MHz}) \mathrm{NMR}$ spectrum of $\mathbf{5 b}\left(\mathrm{THF}-d_{8}, 298 \mathrm{~K}\right)$.

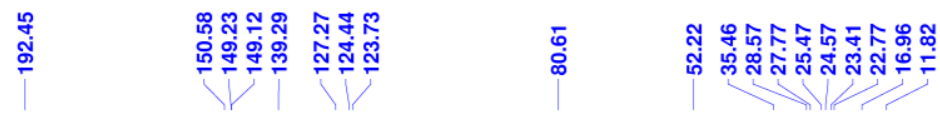

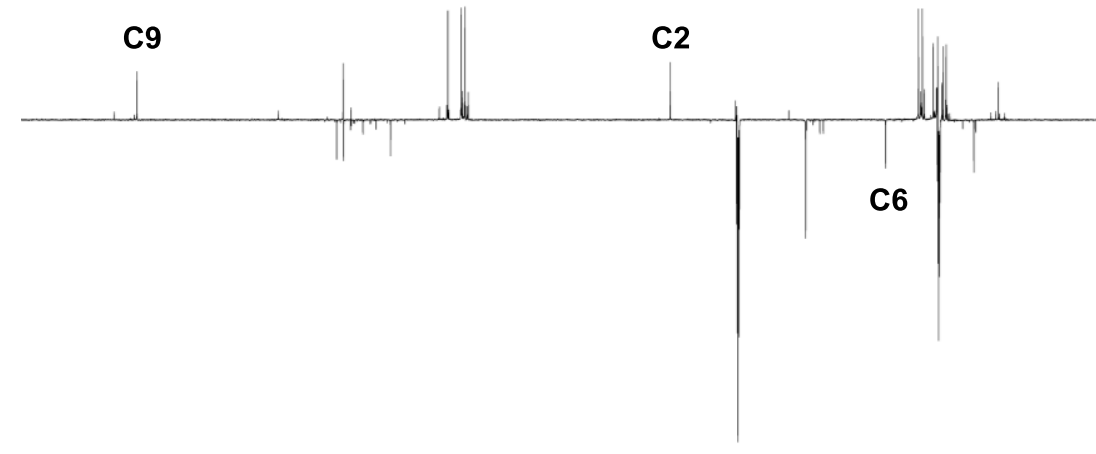

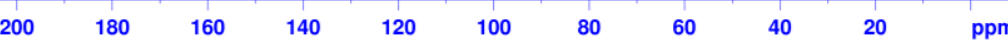

Figure $S 21{ }^{13} \mathrm{C}(150 \mathrm{MHz})$ DEPTQ NMR spectrum of $\mathbf{5 b}\left(\mathrm{THF}-d_{8}, 298 \mathrm{~K}\right)$. 


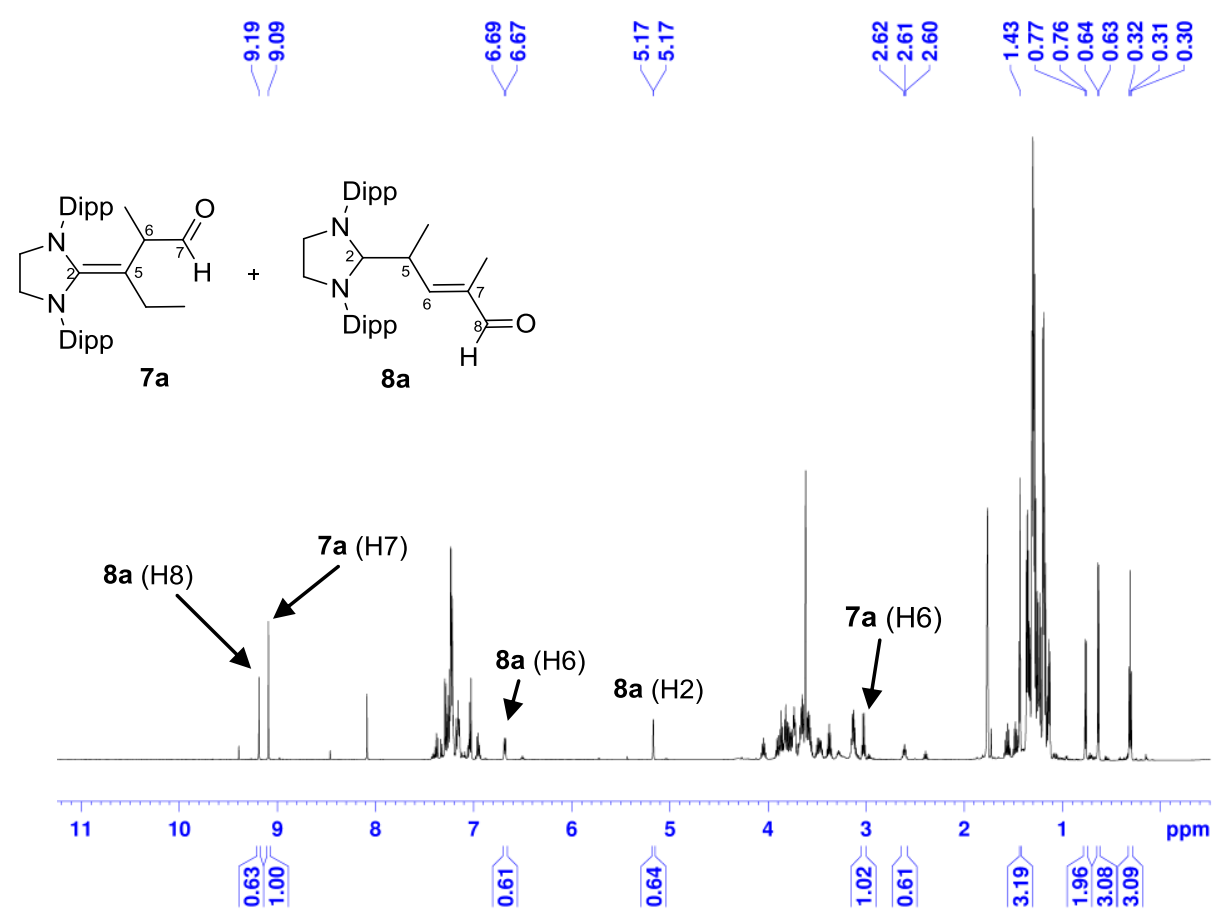

Figure S22 ${ }^{1} \mathrm{H}(600 \mathrm{MHz})$ NMR spectrum of containing mixture of $7 \mathbf{a}$ and $\mathbf{8 a}\left(\mathrm{THF}-d_{8}, 298\right.$ $\mathrm{K})$.
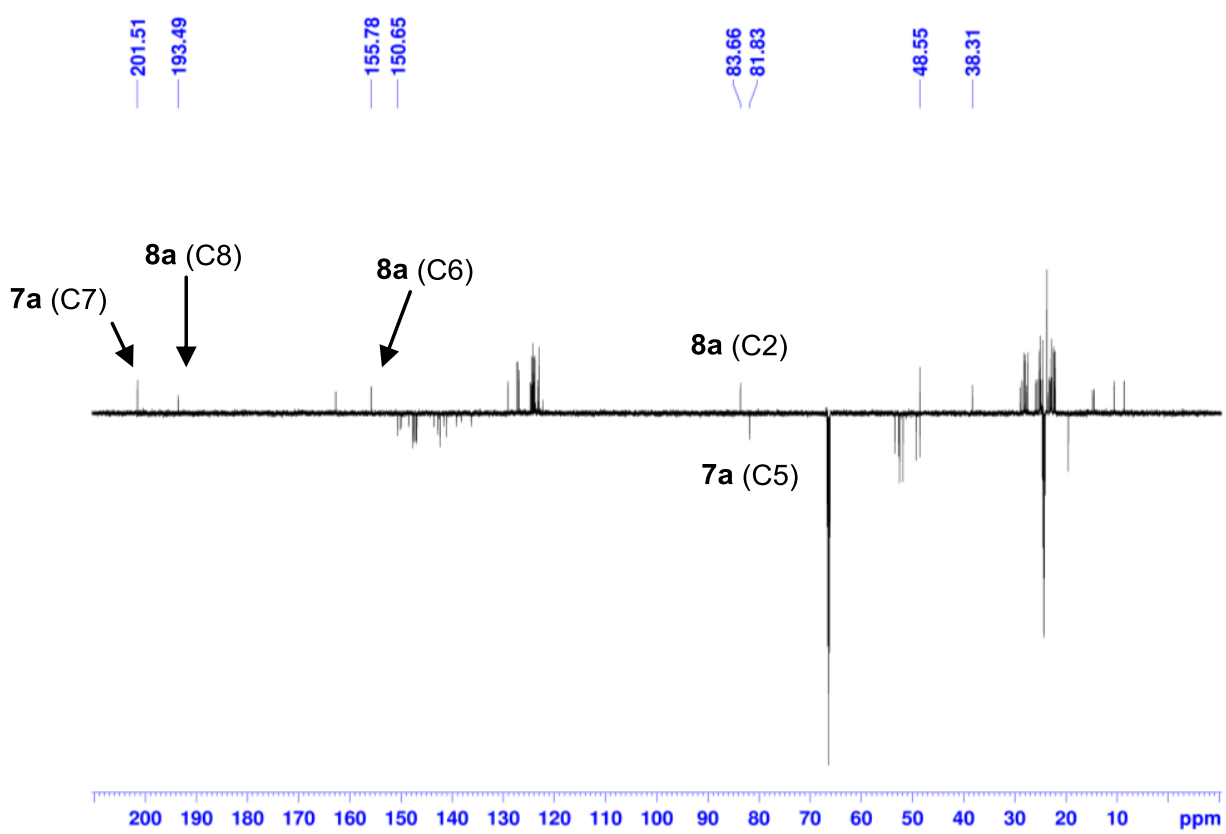

Figure $\mathbf{S 2 3}{ }^{13} \mathrm{C}(150 \mathrm{MHz})$ DEPTQ NMR spectrum of 7a and $\mathbf{8 a}\left(\mathrm{THF}-d_{8}, 298 \mathrm{~K}\right)$. 


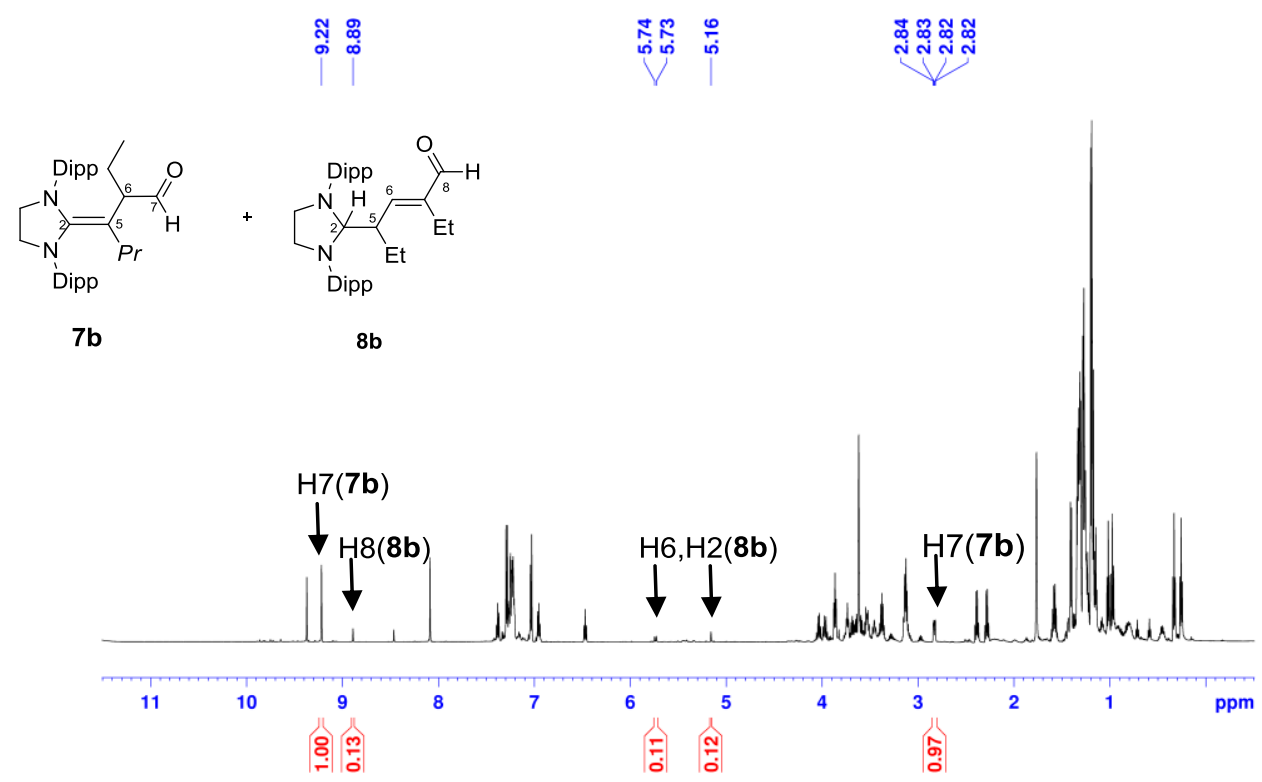

Figure $S 24{ }^{1} \mathrm{H}(600 \mathrm{MHz}) \mathrm{NMR}$ spectrum of $\mathbf{7 b}$ and $\mathbf{8 b}$ (containing 1 and $\left.\mathbf{6 b}\right)\left(\mathrm{THF}-d_{8}, 298\right.$ K).

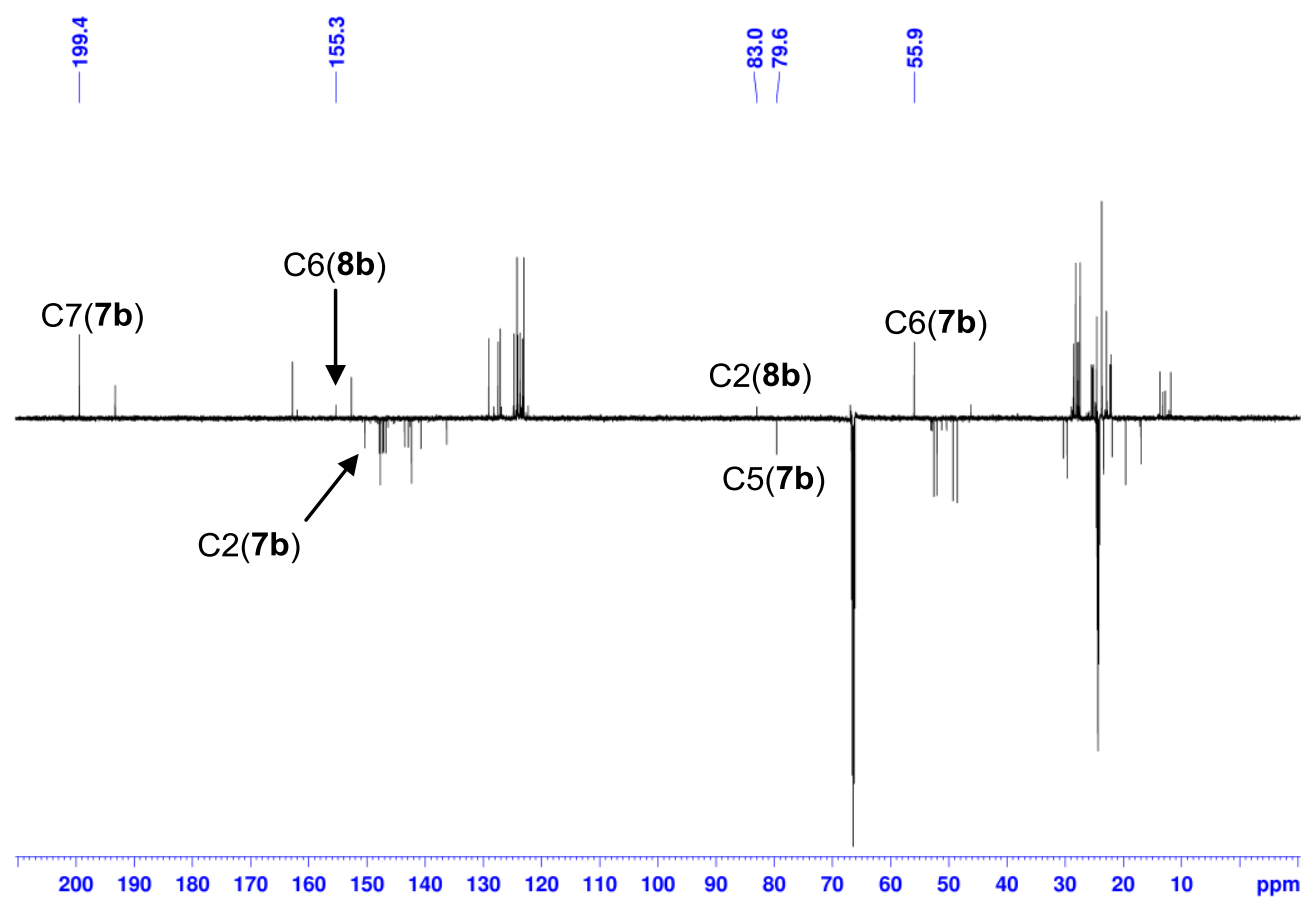

Figure $S 25{ }^{13} \mathrm{C}(150 \mathrm{MHz})$ DEPTQ NMR spectrum of $\mathbf{7 b}$ (containing 1 and $\left.\mathbf{6 b}\right)\left(\mathrm{THF}-\mathrm{d}_{8}, 298\right.$ $\mathrm{K})$. 


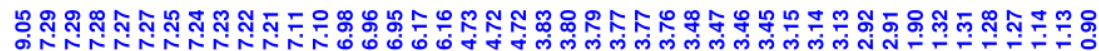

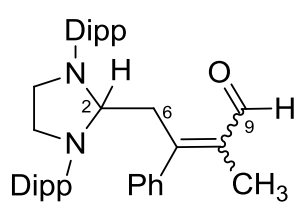

10

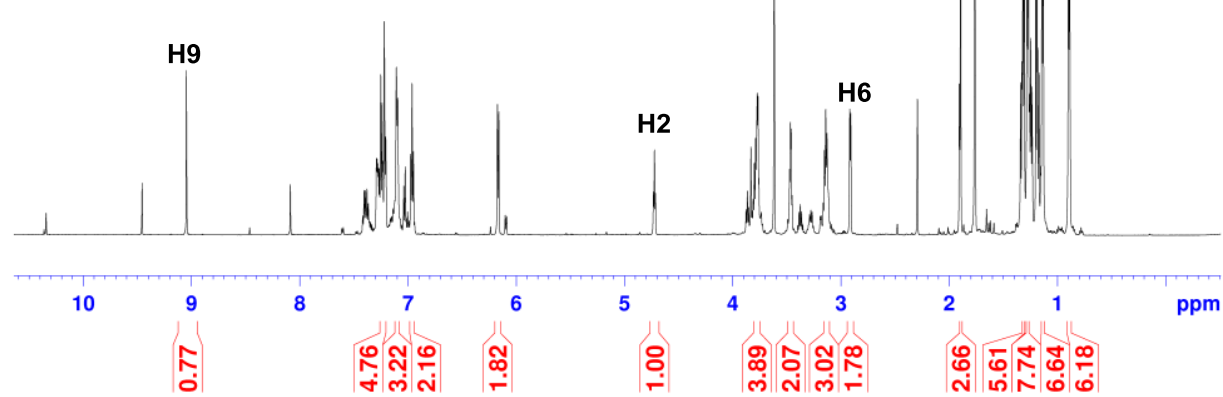

Figure $S 26{ }^{1} \mathrm{H}(600 \mathrm{MHz}) \mathrm{NMR}$ spectrum of 10 (containing $E / Z$ mixture of aldehyde 9) (THF$\left.d_{8}, 298 \mathrm{~K}\right)$.
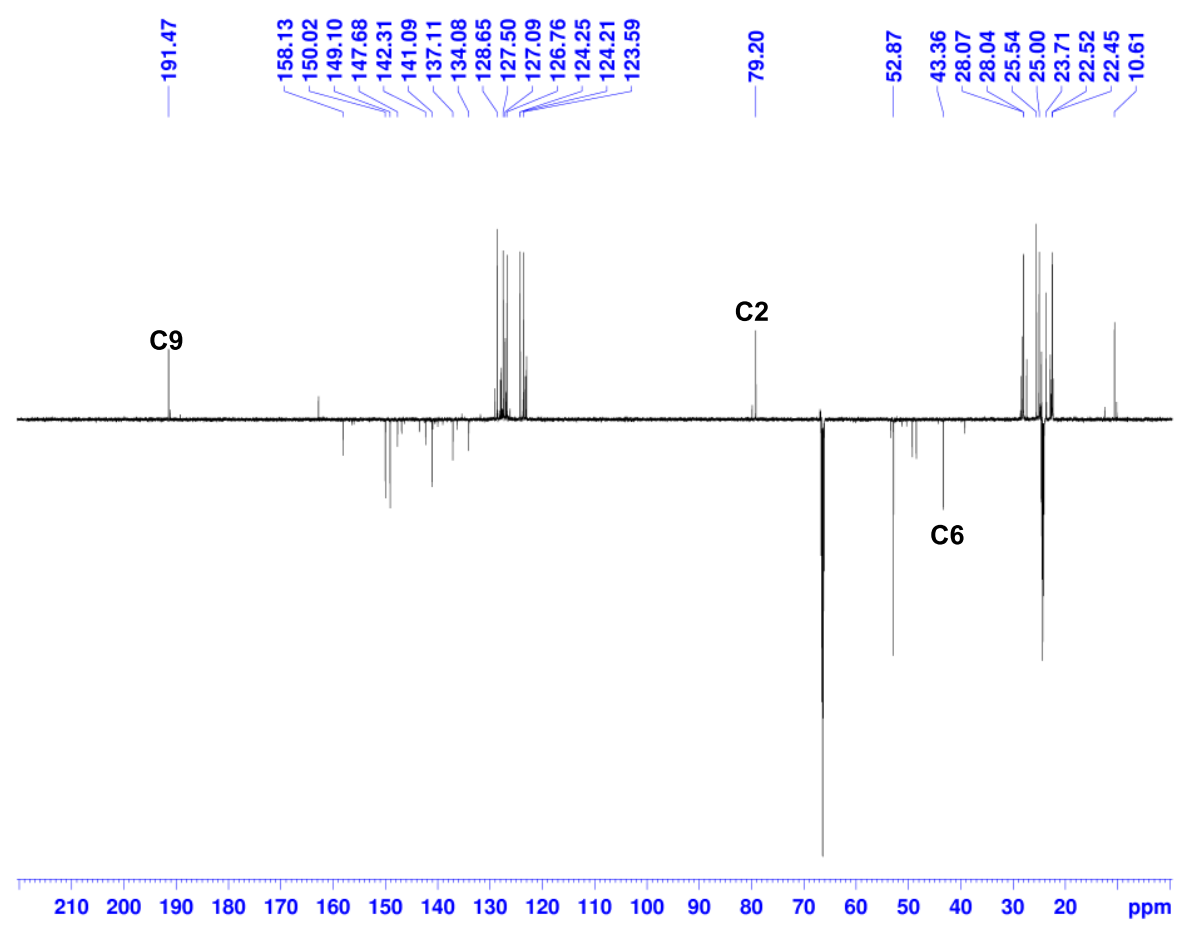

Figure $S 27{ }^{13} \mathrm{C}(150 \mathrm{MHz})$ DEPTQ NMR spectrum of 10 (containing $E / Z$ mixture of aldehyde 9) (THF-d, $298 \mathrm{~K})$. 


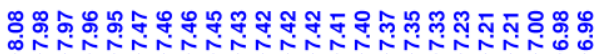

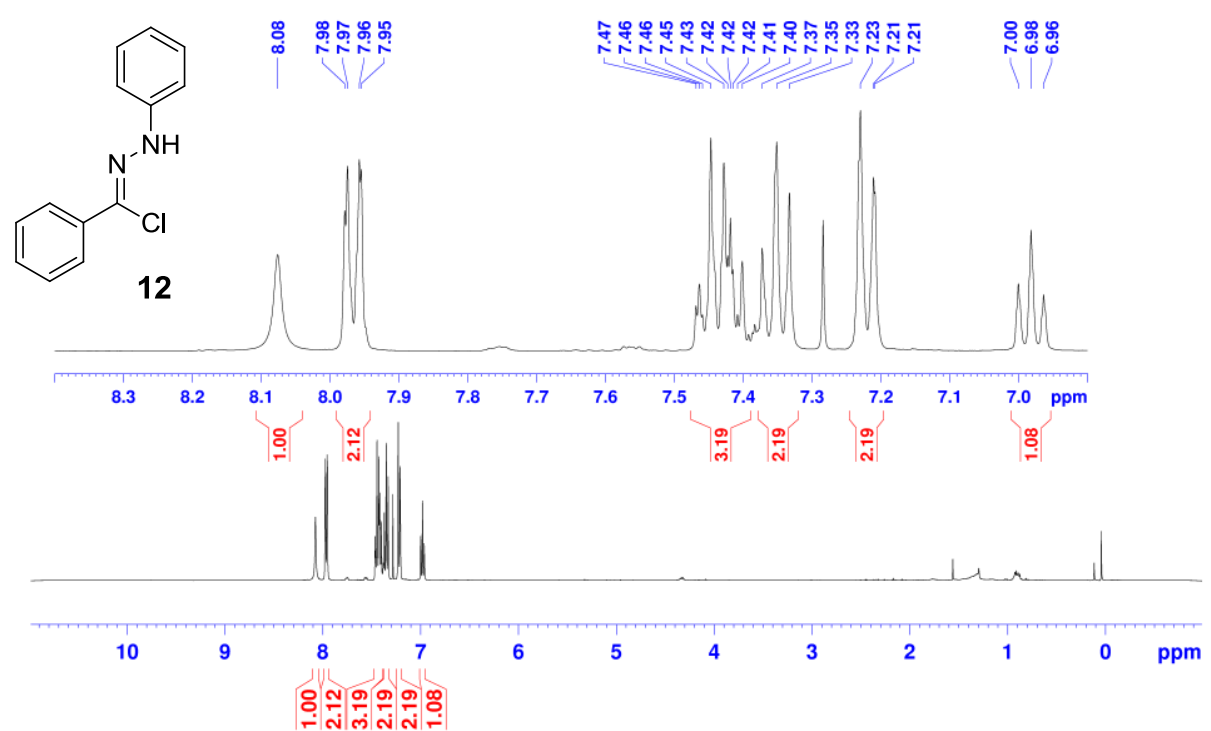

Figure S28 ${ }^{1} \mathrm{H}(600 \mathrm{MHz}) \mathrm{NMR}$ spectrum of $12\left(\mathrm{CDCl}_{3}, 298 \mathrm{~K}\right)$.

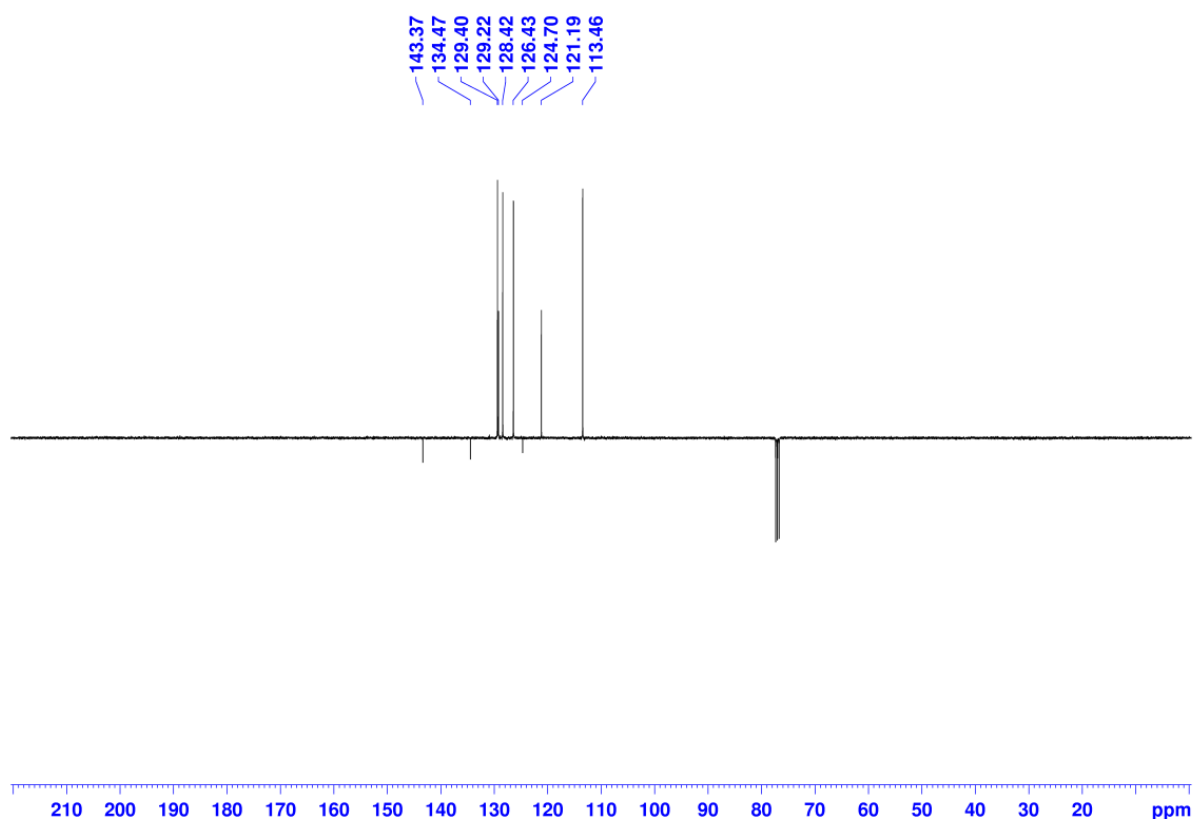

Figure S29 ${ }^{13} \mathrm{C}(150 \mathrm{MHz})$ DEPTQ NMR spectrum of $12\left(\mathrm{CDCl}_{3}, 298 \mathrm{~K}\right)$. 


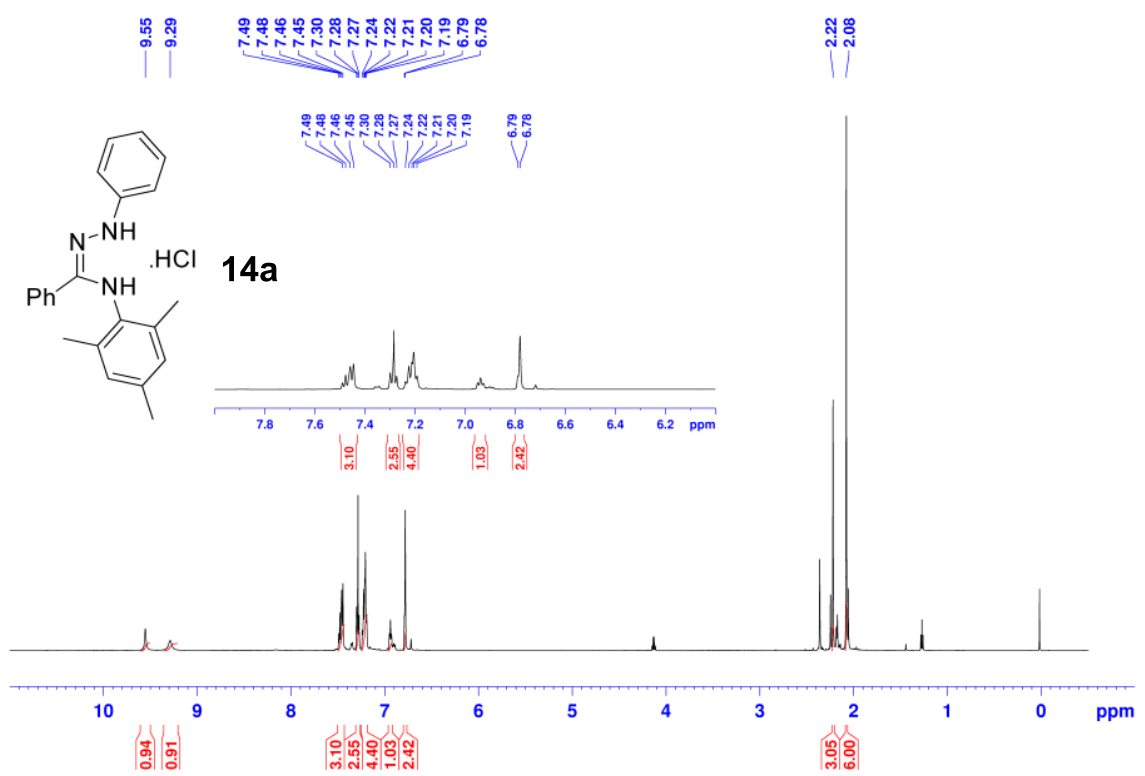

Figure $\mathrm{S} 30^{1} \mathrm{H}(600 \mathrm{MHz}) \mathrm{NMR}$ spectrum of $14 \mathrm{a}\left(\mathrm{CDCl}_{3}, 298 \mathrm{~K}\right)$.

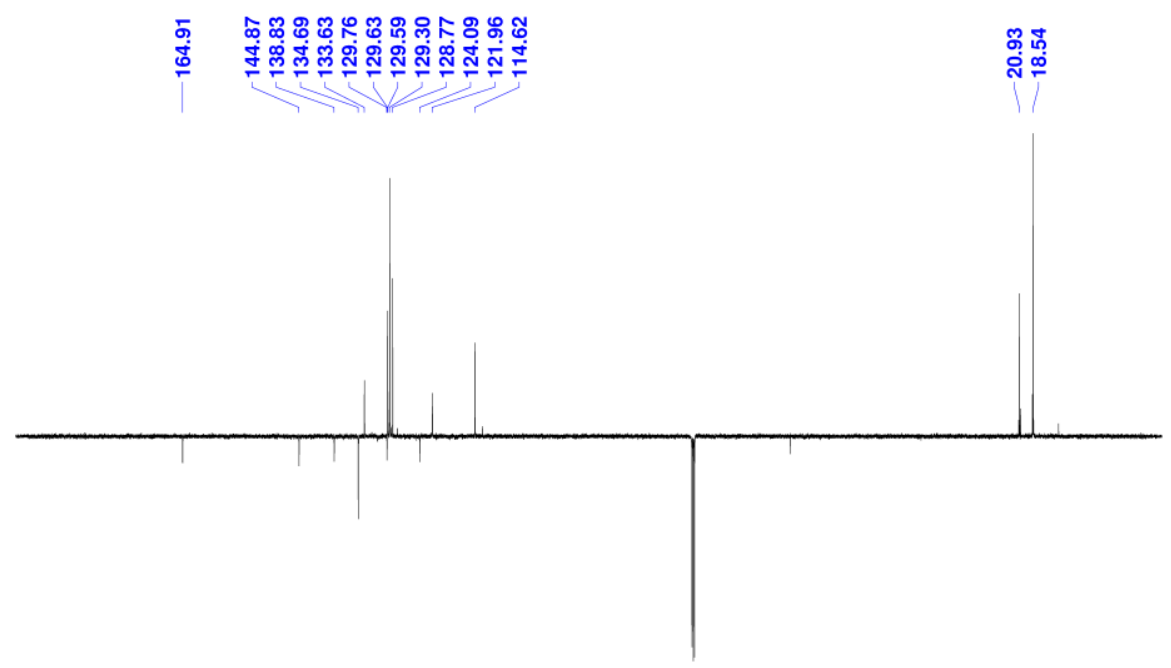

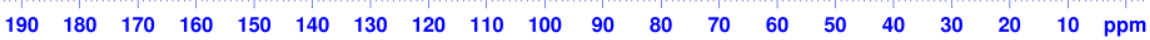

Figure $531{ }^{13} \mathrm{C}(150 \mathrm{MHz})$ DEPTQ NMR spectrum of 14a $\left(\mathrm{CDCl}_{3}, 298 \mathrm{~K}\right)$. 

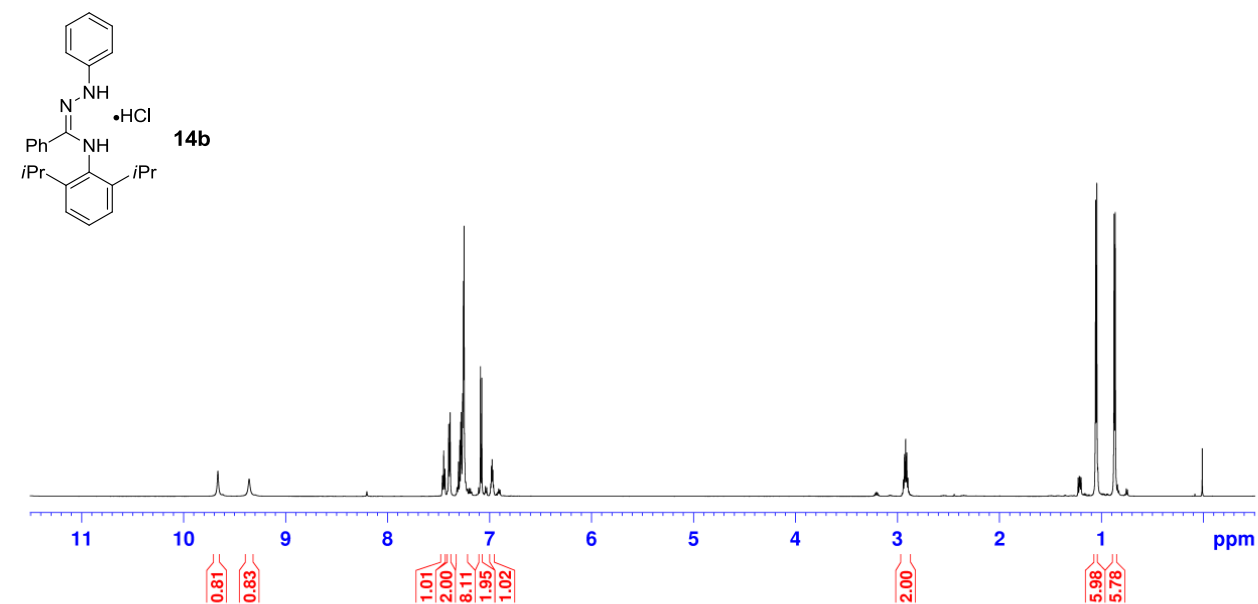

Figure $S 32{ }^{1} \mathrm{H}(600 \mathrm{MHz}) \mathrm{NMR}$ spectrum of $14 \mathbf{b}\left(\mathrm{CDCl}_{3}, 298 \mathrm{~K}\right)$.

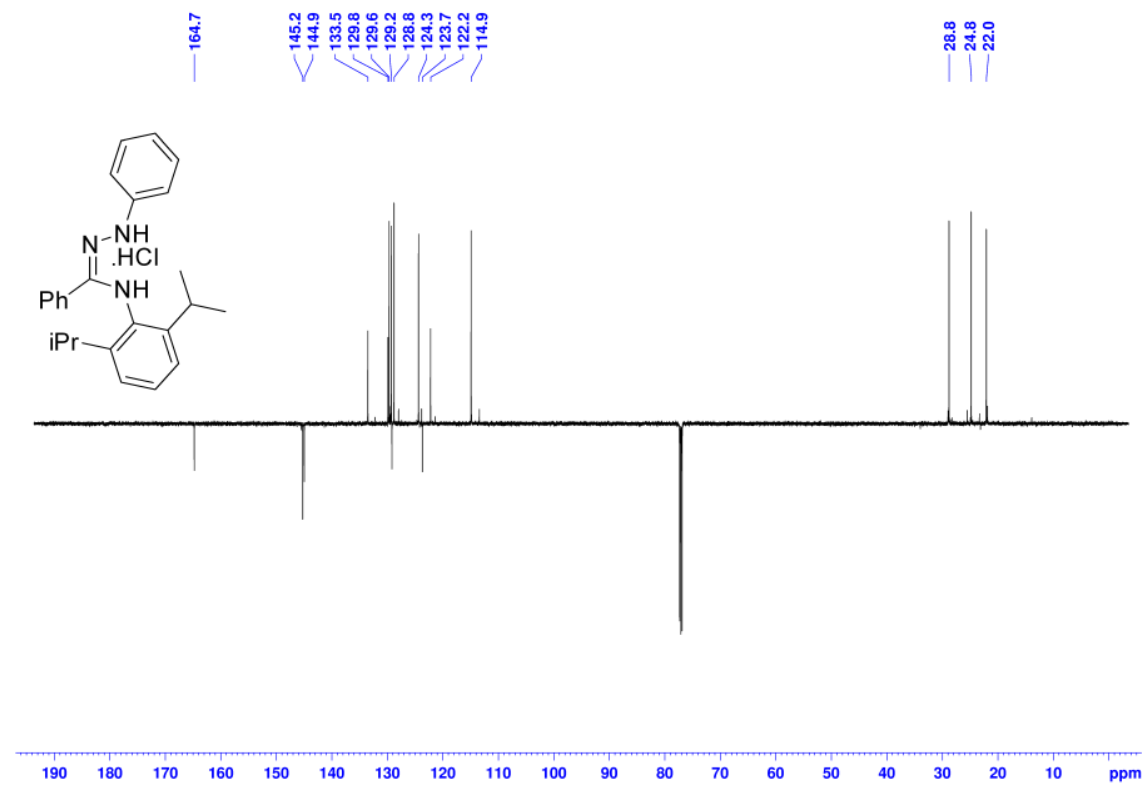

Figure $S 33{ }^{13} \mathrm{C}(150 \mathrm{MHz})$ DEPTQ NMR spectrum of 14b $\left(\mathrm{CDCl}_{3}, 298 \mathrm{~K}\right)$. 

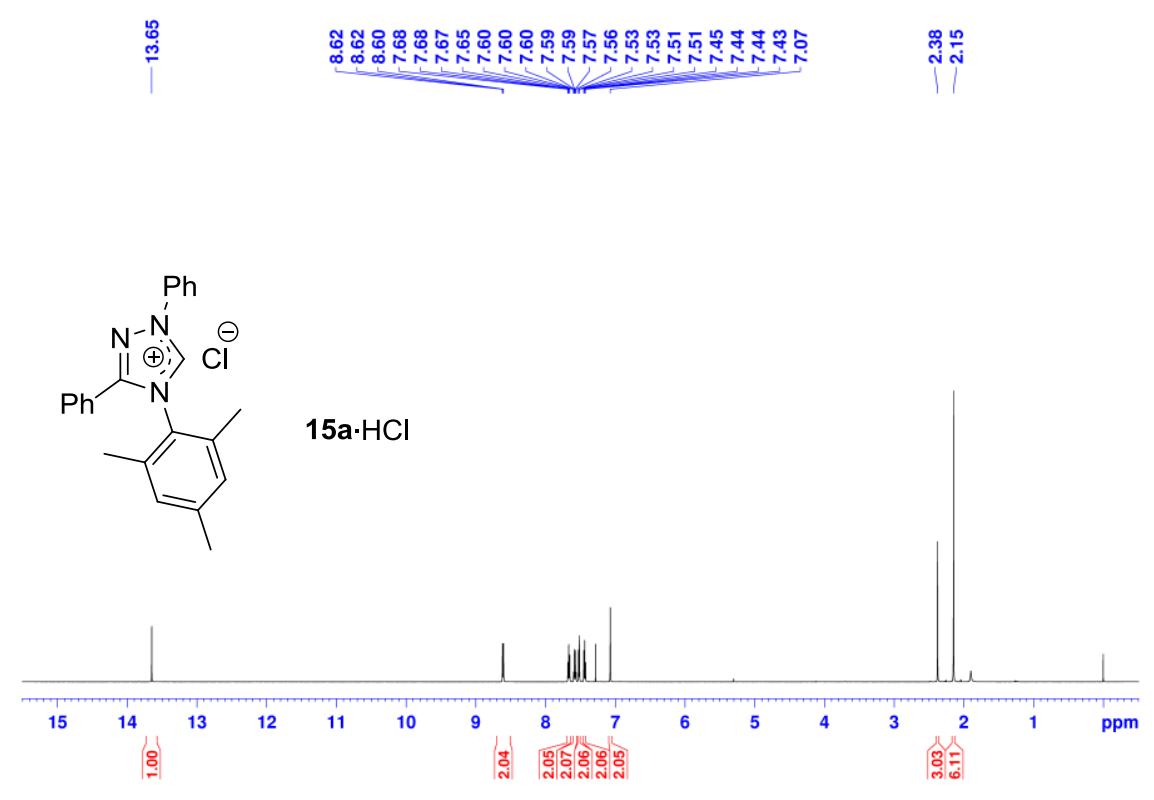

Figure $\mathrm{S} 34{ }^{1} \mathrm{H}(600 \mathrm{MHz}) \mathrm{NMR}$ spectrum of $15 \mathrm{a} \cdot \mathrm{HCl}\left(\mathrm{CDCl}_{3}, 298 \mathrm{~K}\right)$.

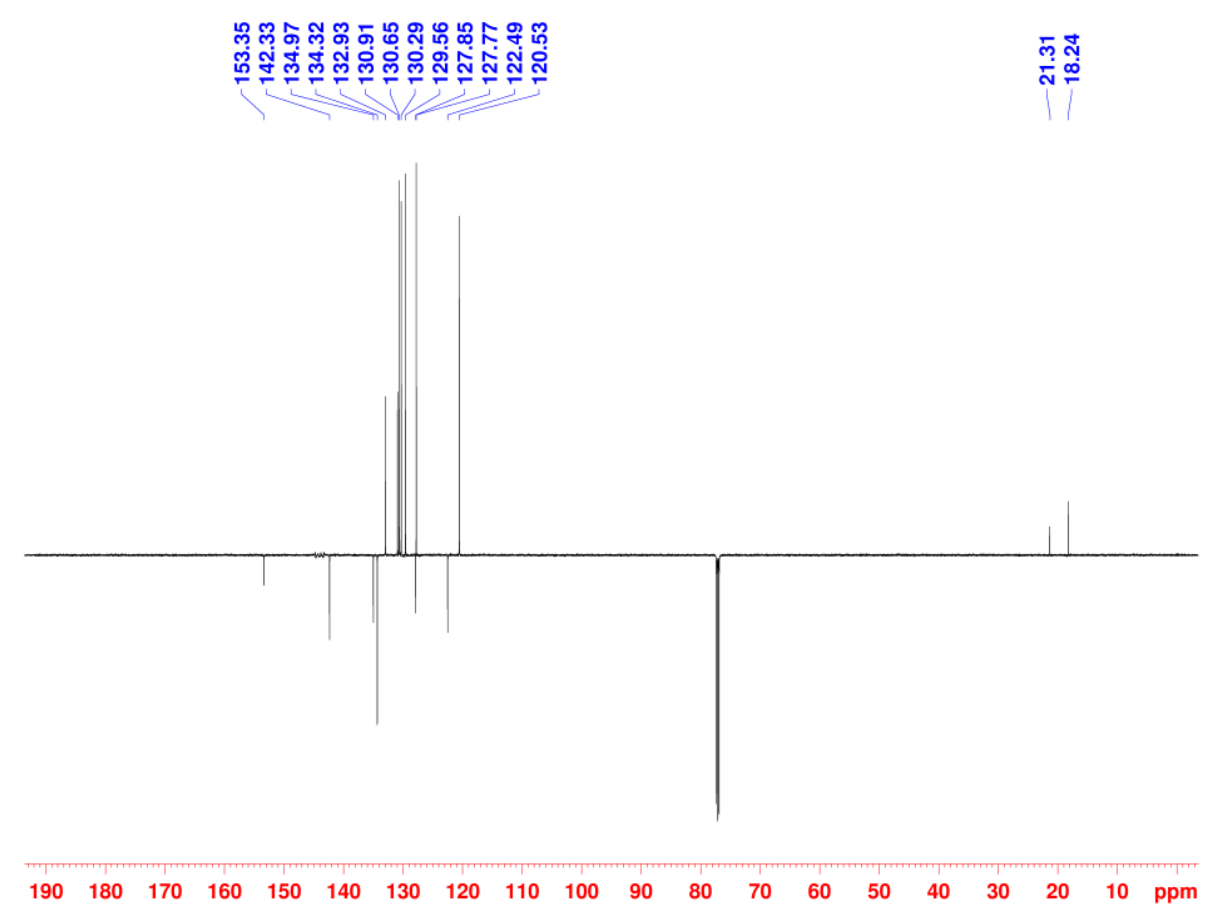

Figure $S 35{ }^{13} \mathrm{C}(150 \mathrm{MHz})$ DEPTQ NMR spectrum of $15 a \cdot H C l\left(\mathrm{CDCl}_{3}, 298 \mathrm{~K}\right)$. 


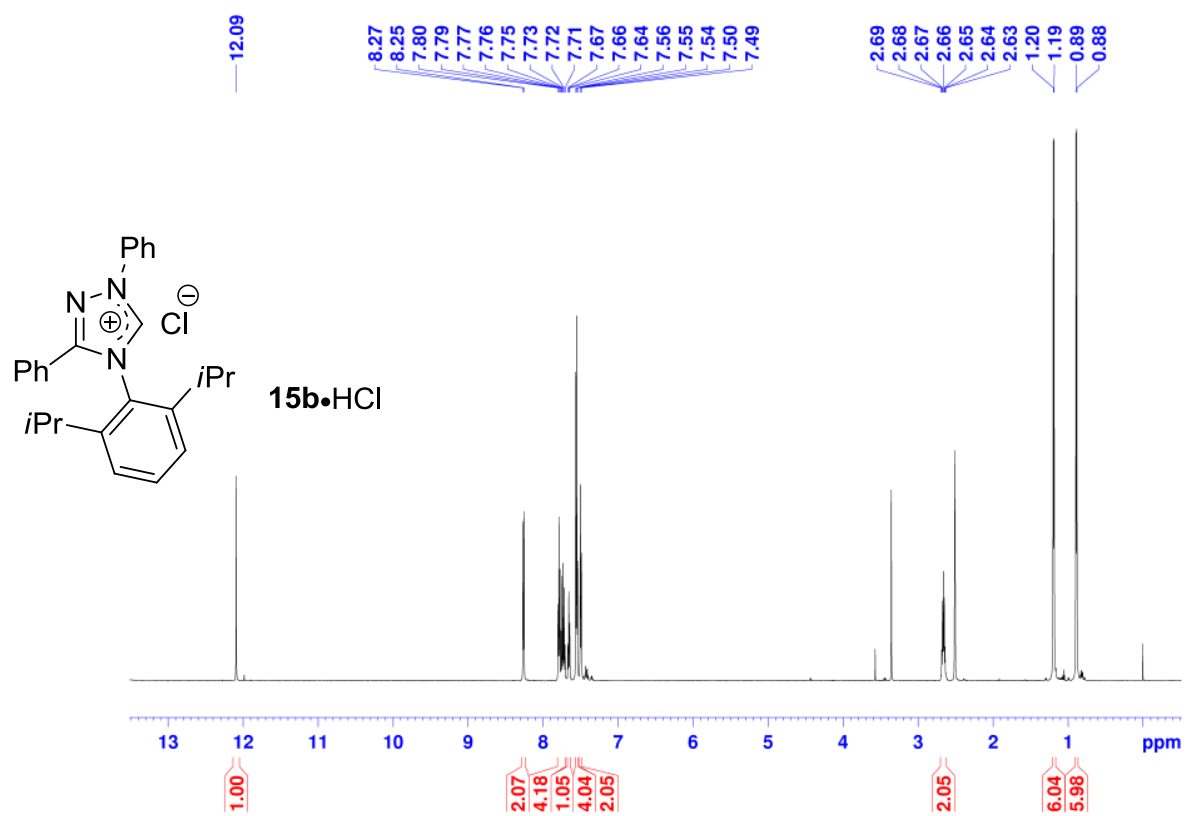

Figure $S 36{ }^{1} \mathrm{H}(600 \mathrm{MHz}) \mathrm{NMR}$ spectrum of $15 \mathrm{~b} \cdot \mathrm{HCl}\left(\mathrm{DMSO}-d_{6}, 298 \mathrm{~K}\right)$.

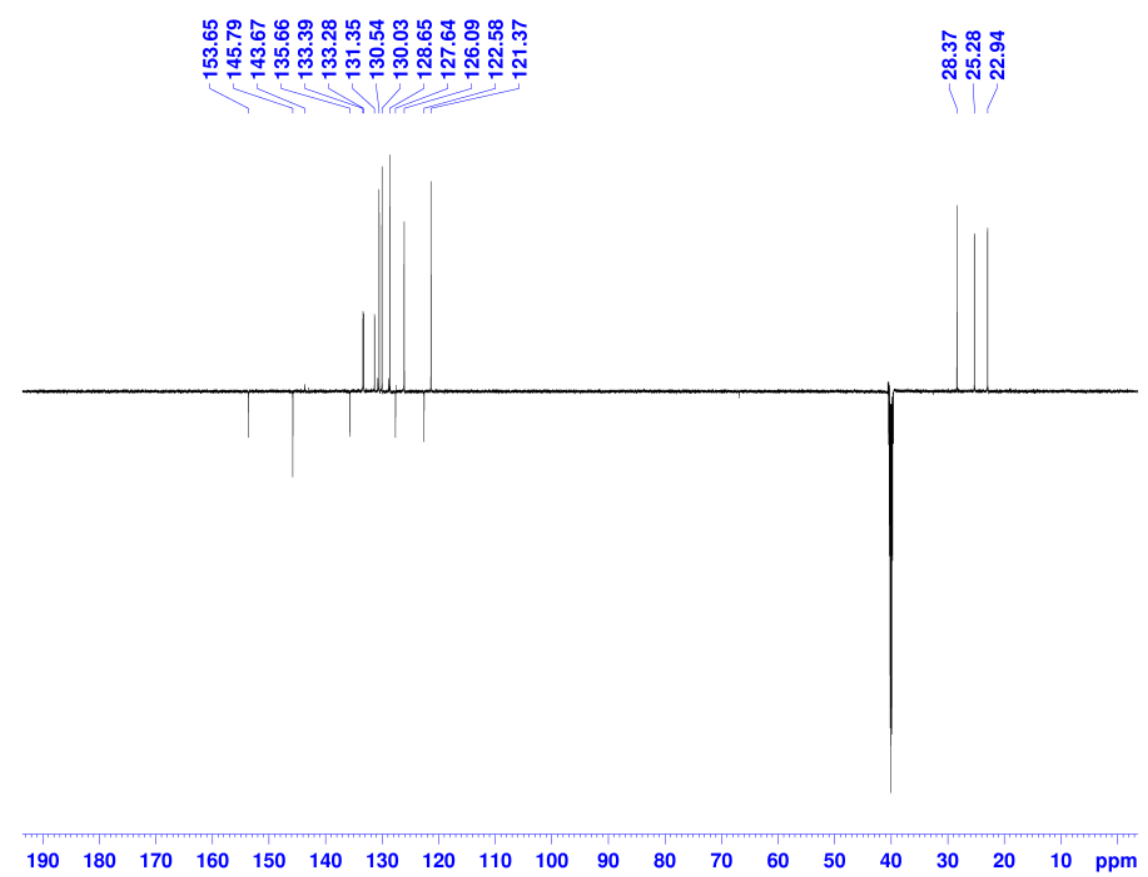

Figure $S 37{ }^{13} \mathrm{C}(150 \mathrm{MHz})$ DEPTQ NMR spectrum of $15 \mathrm{~b} \cdot \mathrm{HCl}\left(\mathrm{DMSO}-d_{6}, 298 \mathrm{~K}\right)$. 


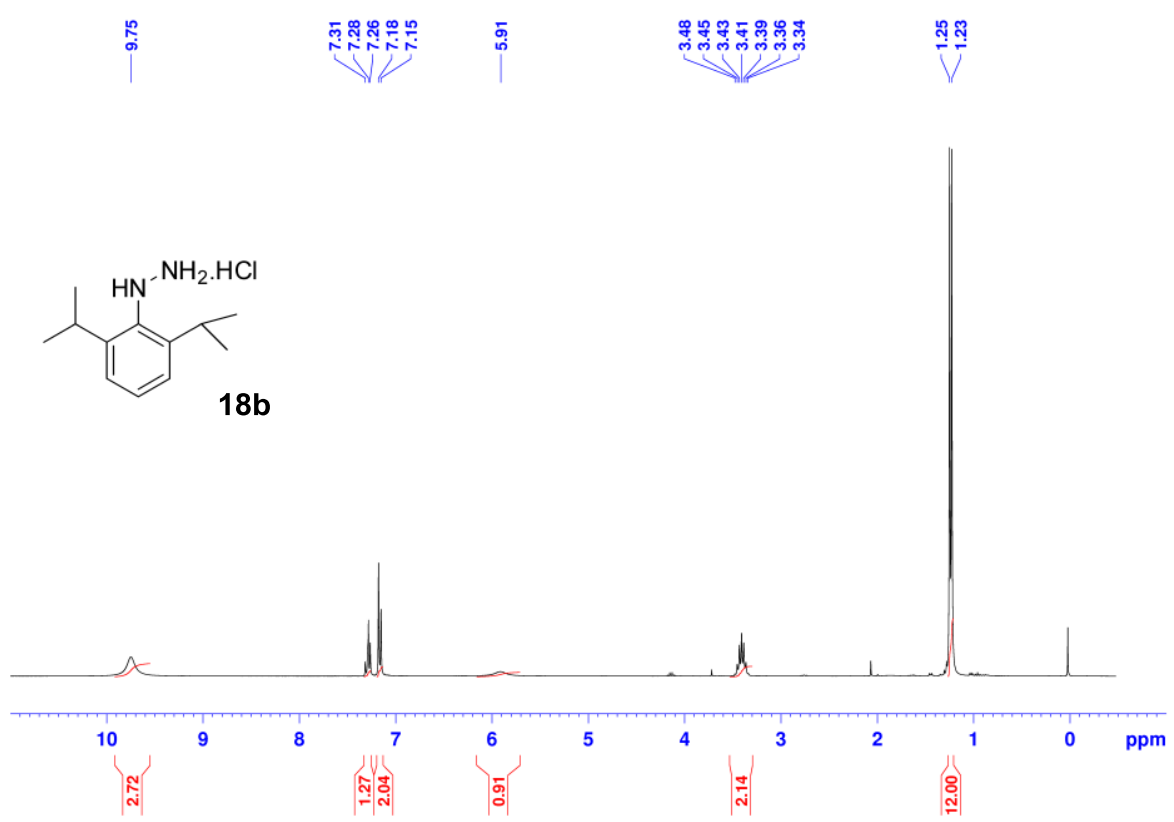

Figure $S 38{ }^{1} \mathrm{H}(600 \mathrm{MHz})$ NMR spectrum of $18 \mathrm{~b}\left(\mathrm{DMSO}-d_{6}, 298 \mathrm{~K}\right)$.

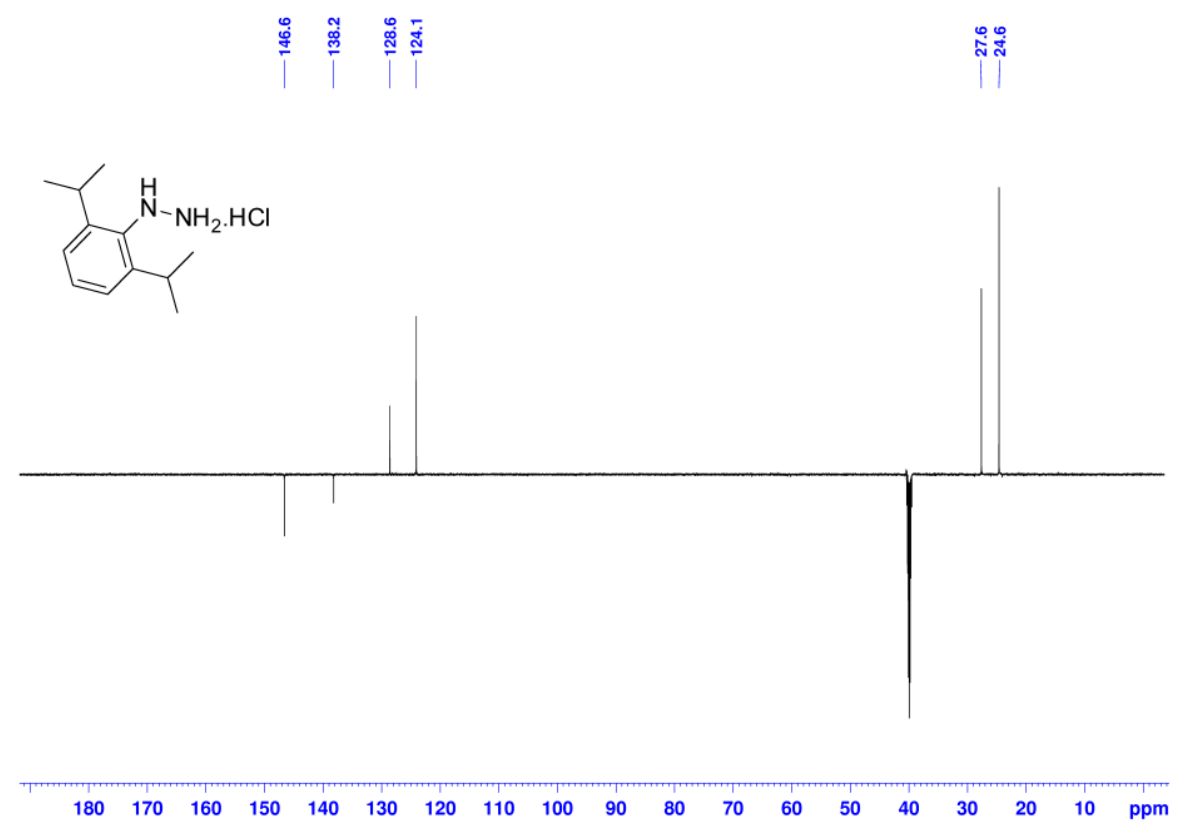

Figure $S 39{ }^{13} \mathrm{C}(150 \mathrm{MHz})$ DEPTQ NMR spectrum of 18b (DMSO- $\left.d_{6}, 298 \mathrm{~K}\right)$. 


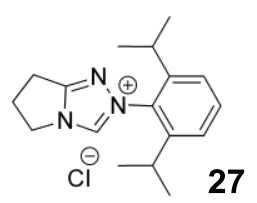

27

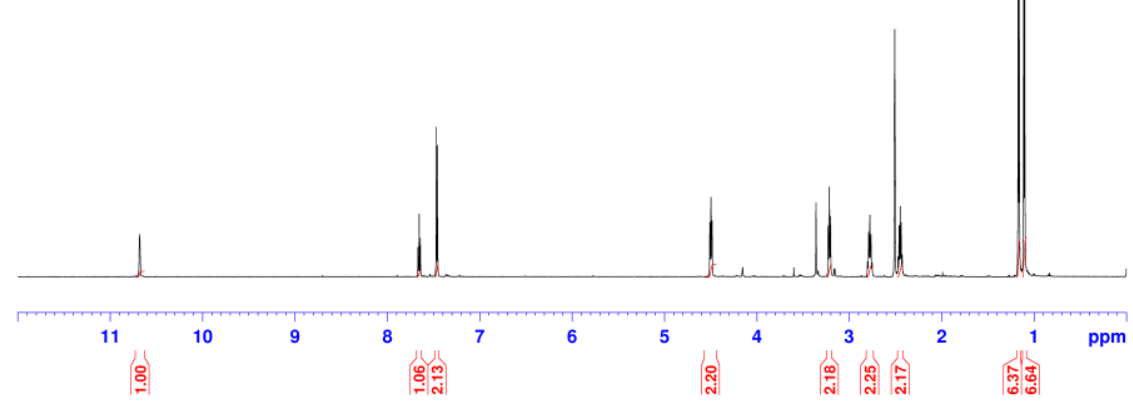

Figure $\mathbf{S 4 0}{ }^{1} \mathrm{H}(600 \mathrm{MHz}) \mathrm{NMR}$ spectrum of $27\left(\mathrm{DMSO}-d_{6}, 298 \mathrm{~K}\right)$.

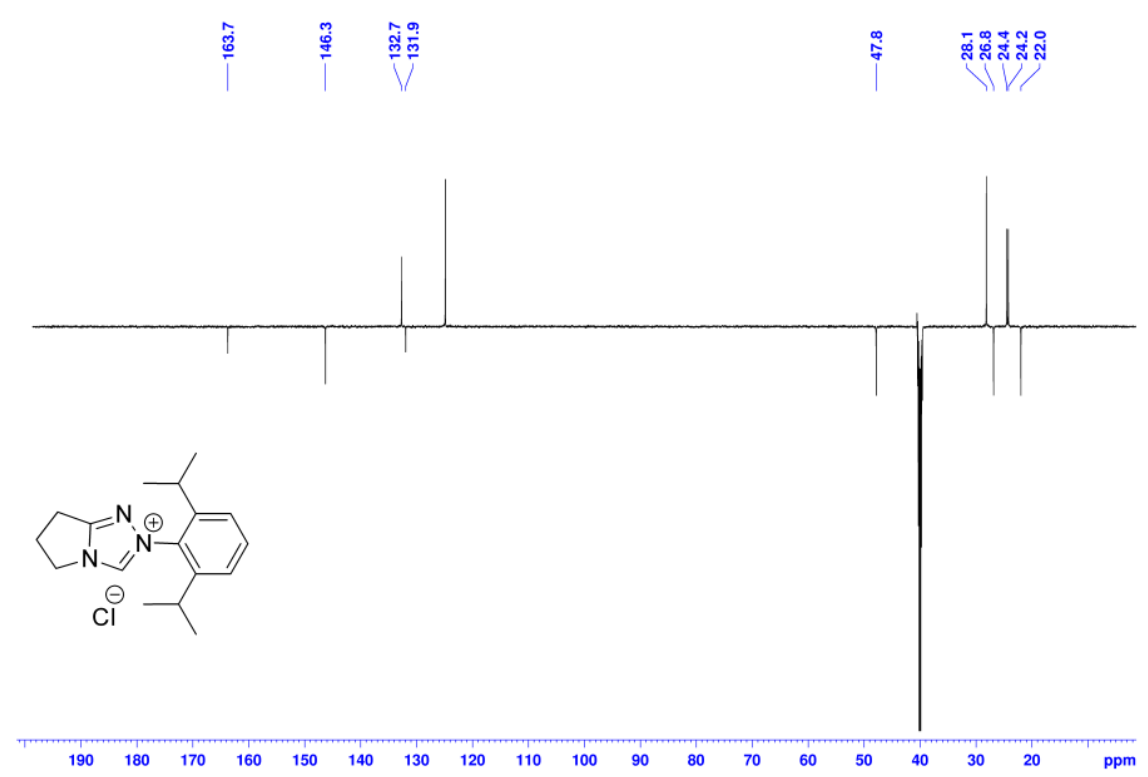

Figure $541{ }^{13} \mathrm{C}(150 \mathrm{MHz})$ DEPTQ NMR spectrum of 27 ([D $]$ DMSO, $\left.298 \mathrm{~K}\right)$. 


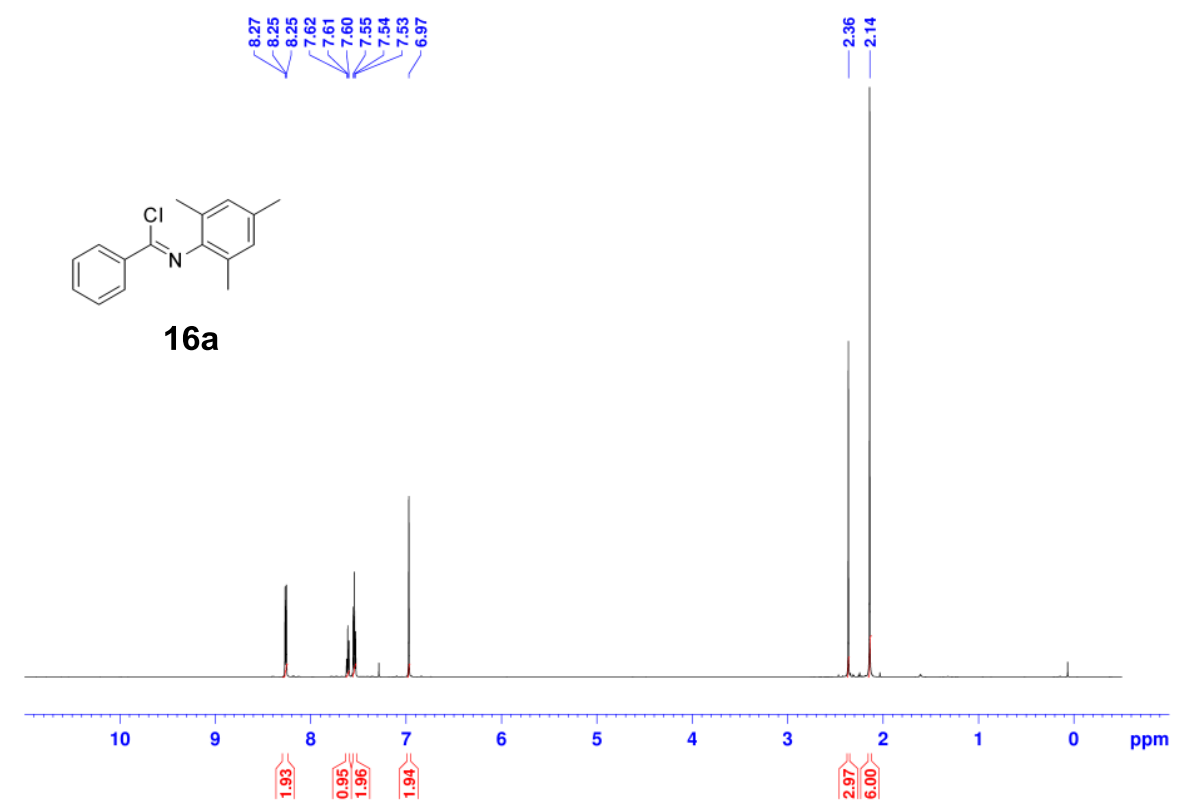

Figure $S 42{ }^{1} \mathrm{H}(600 \mathrm{MHz}) \mathrm{NMR}$ spectrum of $16 \mathrm{a}\left(\mathrm{CDCl}_{3}, 298 \mathrm{~K}\right)$.

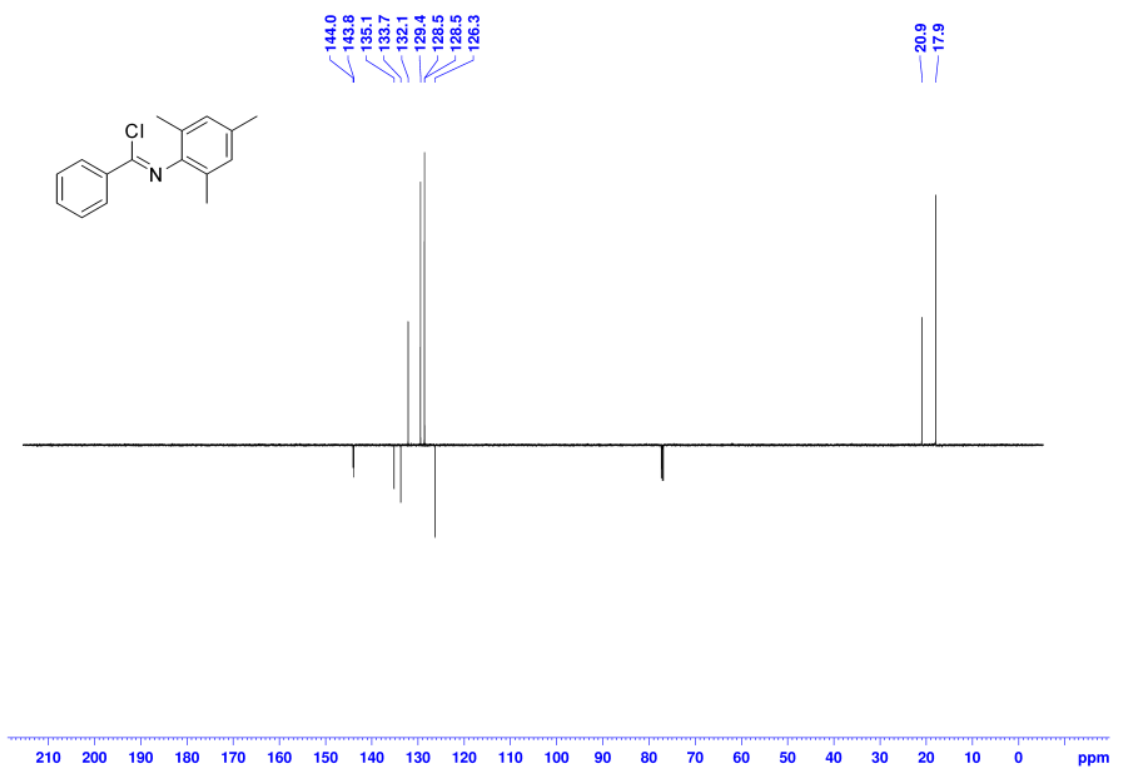

Figure $\mathbf{S 4 3}{ }^{13} \mathrm{C}(150 \mathrm{MHz})$ DEPTQ NMR spectrum of $\mathbf{1 6 a}\left(\mathrm{CDCl}_{3}, 298 \mathrm{~K}\right)$. 


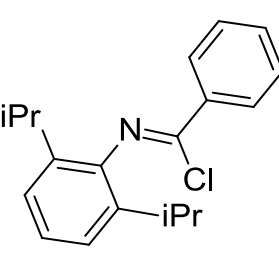

$16 b$

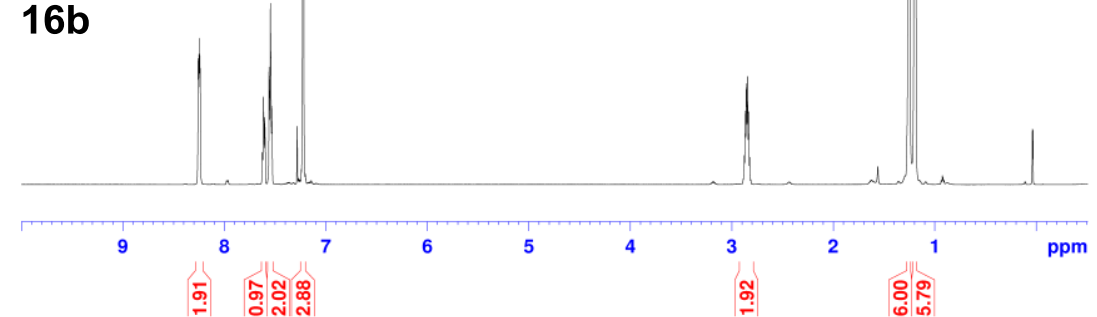

Figure $\mathbf{S 4 4}{ }^{1} \mathrm{H}(600 \mathrm{MHz}) \mathrm{NMR}$ spectrum of $\mathbf{1 6 b}\left(\mathrm{CDCl}_{3}, 298 \mathrm{~K}\right)$.

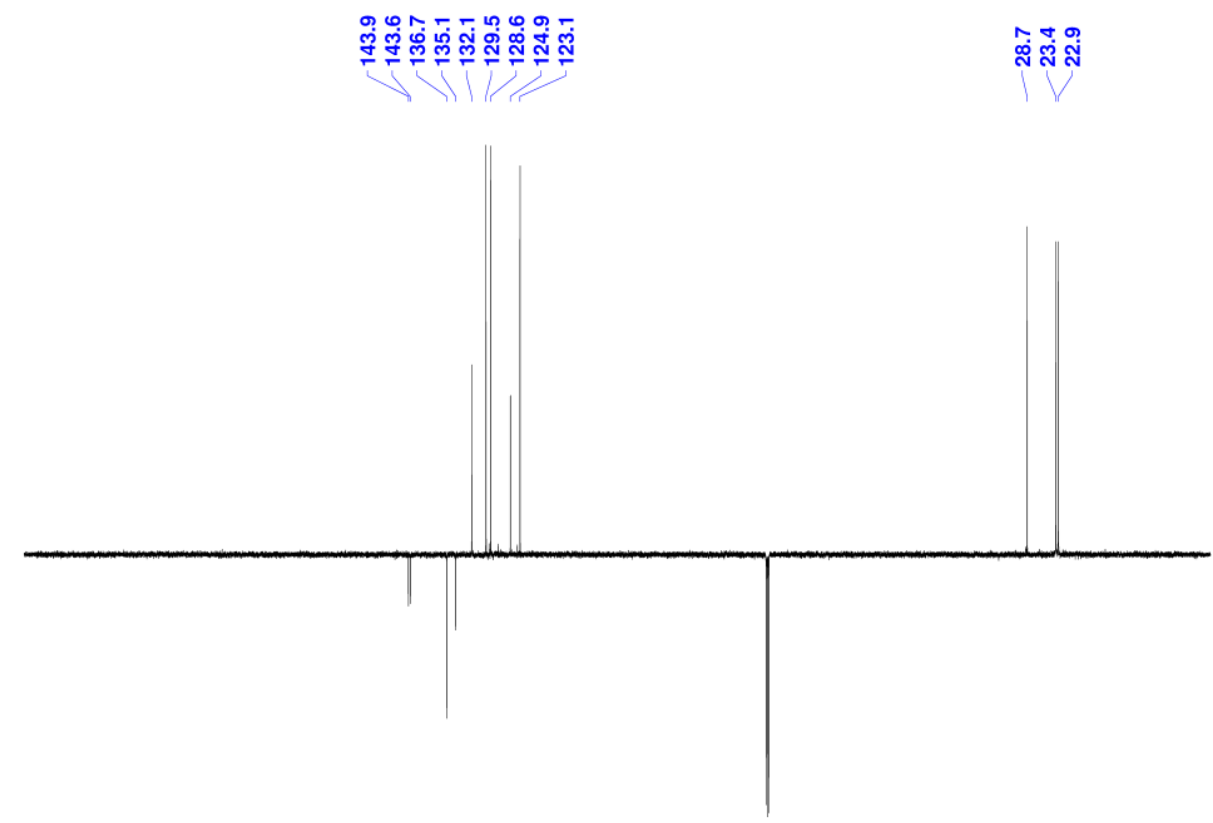

$\begin{array}{llllllllllllllllllllll}210 & 200 & 190 & 180 & 170 & 160 & 150 & 140 & 130 & 120 & 110 & 100 & 90 & 80 & 70 & 60 & 50 & 40 & 30 & 20 & 10 & \mathrm{ppm}\end{array}$

Figure $S 45{ }^{13} \mathrm{C}(150 \mathrm{MHz})$ DEPTQ NMR spectrum of $16 \mathrm{~b}\left(\mathrm{CDCl}_{3}, 298 \mathrm{~K}\right)$. 


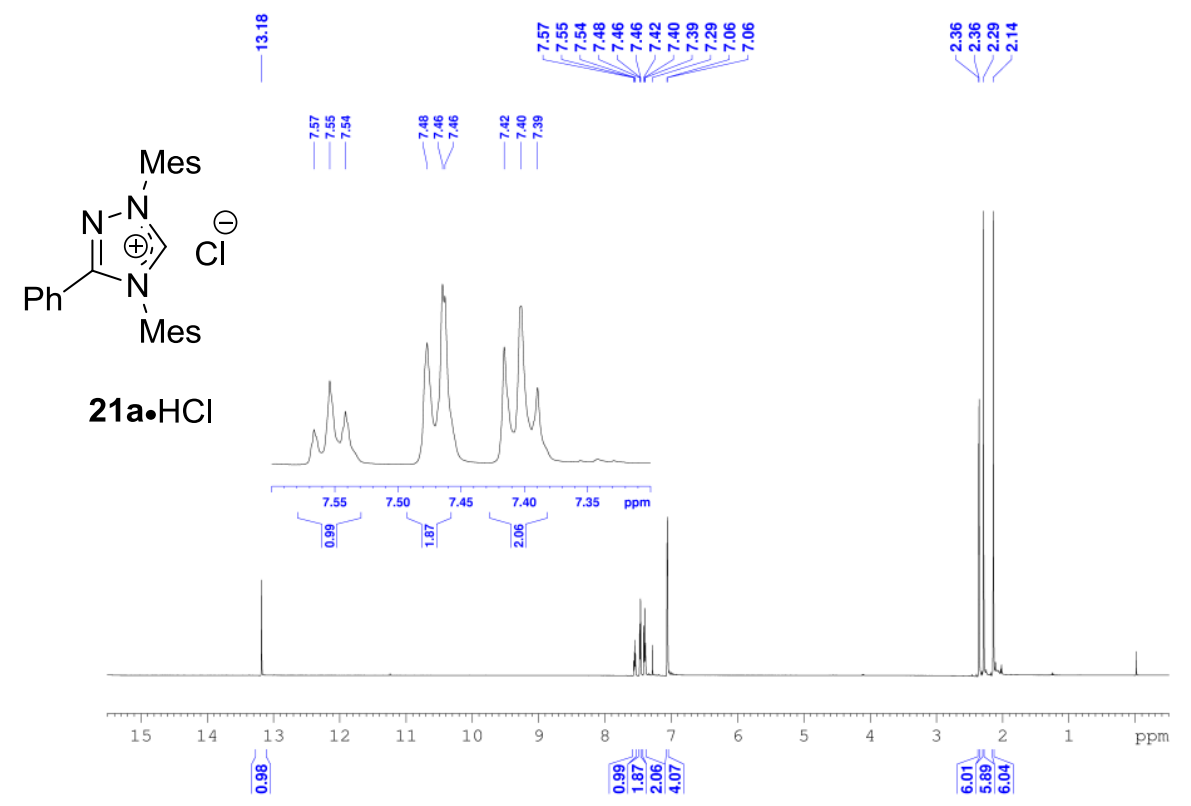

Figure $\mathbf{S 4 6}{ }^{1} \mathrm{H}(600 \mathrm{MHz}) \mathrm{NMR}$ spectrum of $21 \mathrm{a} \cdot \mathrm{HCl}\left(\mathrm{CDCl}_{3}, 298 \mathrm{~K}\right)$.

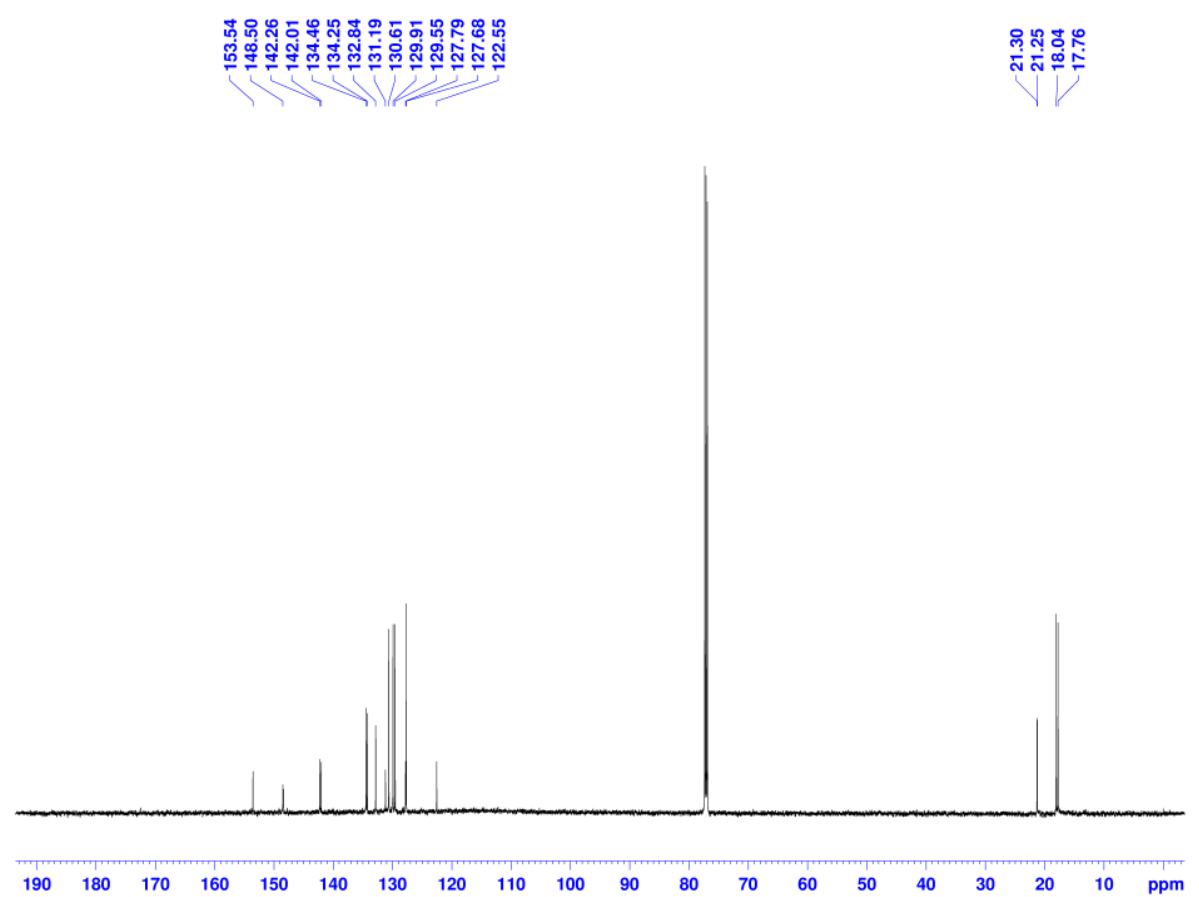

Figure $\mathbf{S 4 7} \mathbf{7}^{13} \mathrm{C}(150 \mathrm{MHz})$ DEPTQ NMR spectrum of $\mathbf{2 1 a} \cdot \mathrm{HCl}\left(\mathrm{CDCl}_{3}, 298 \mathrm{~K}\right)$. 


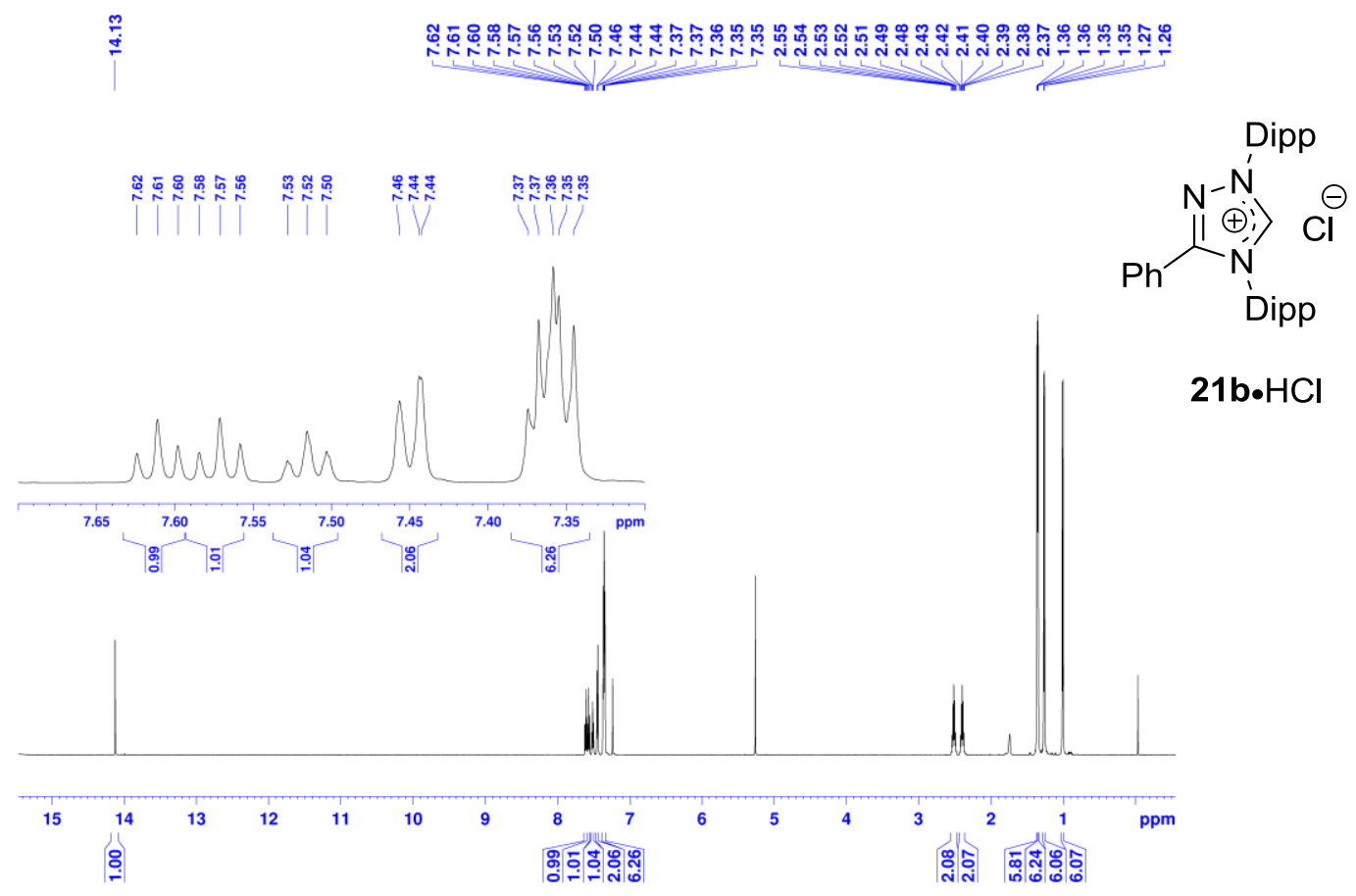

Figure $\mathbf{S 4 8}{ }^{1} \mathrm{H}(600 \mathrm{MHz}) \mathrm{NMR}$ spectrum of $\mathbf{2 1} \mathbf{b} \cdot \mathrm{HCl}\left(\mathrm{CDCl}_{3}, 298 \mathrm{~K}\right)$.

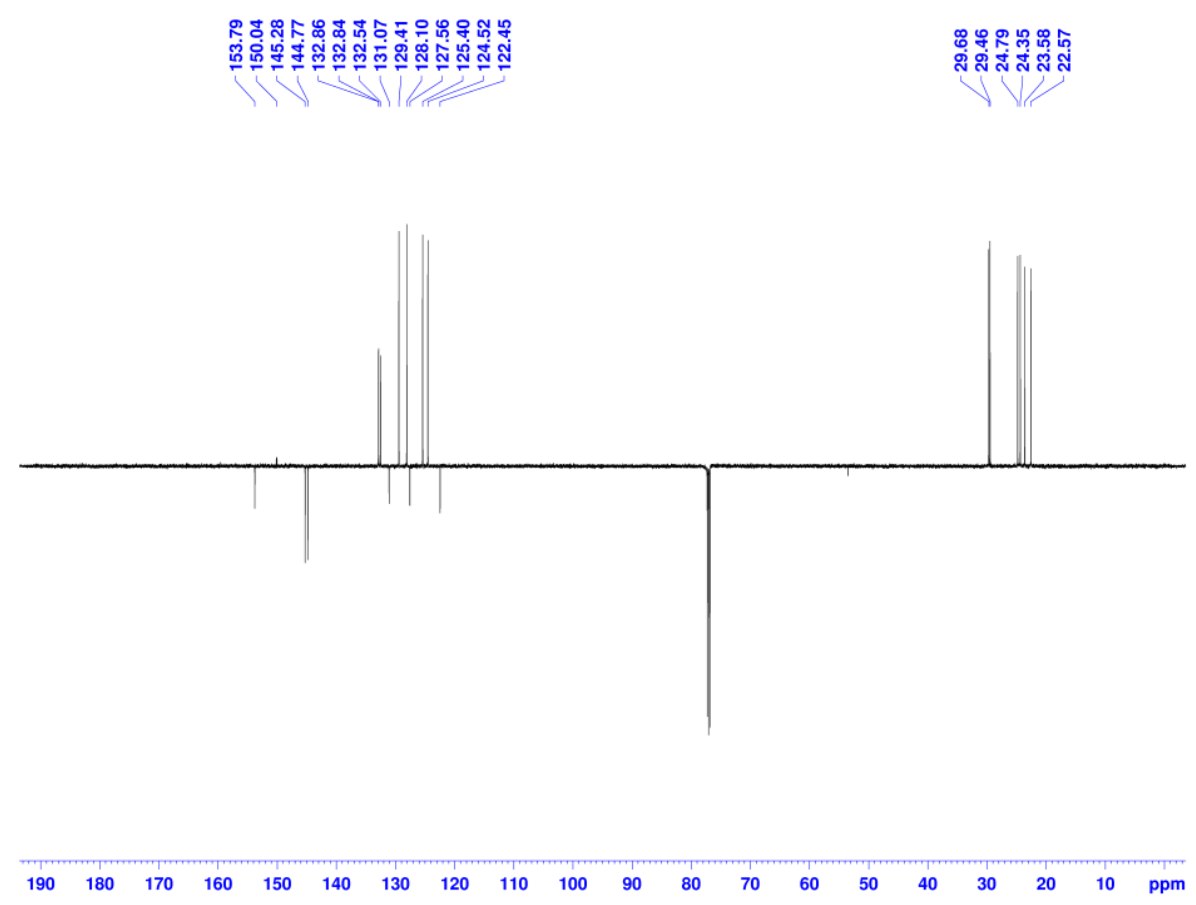

Figure $\mathbf{S 4 9}{ }^{13} \mathrm{C}(150 \mathrm{MHz})$ DEPTQ NMR spectrum of $\mathbf{2 1 b} \cdot \mathrm{HCl}\left(\mathrm{CDCl}_{3}, 298 \mathrm{~K}\right)$. 


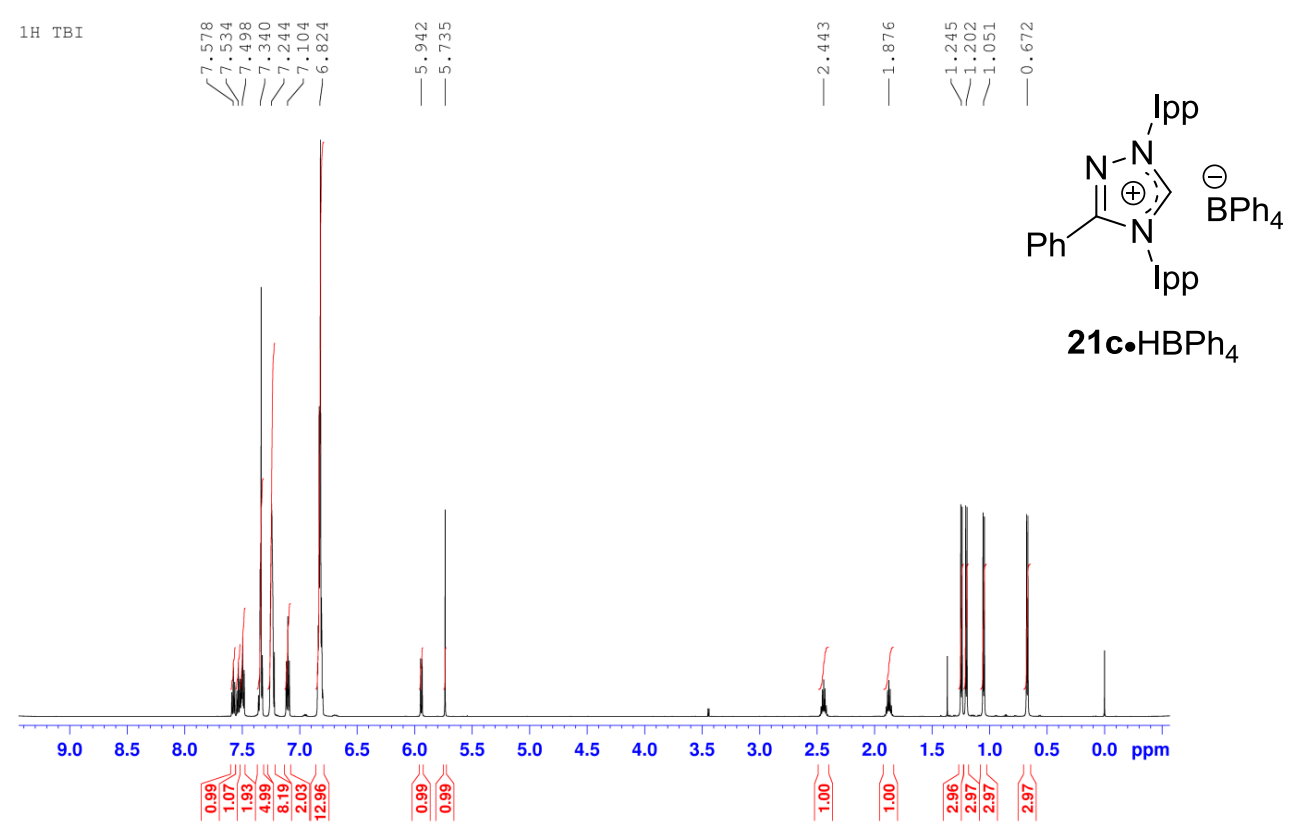

Figure $\mathrm{S}^{1} \mathrm{C}^{1} \mathrm{H}(600 \mathrm{MHz}) \mathrm{NMR}$ spectrum of $21 \mathrm{c} \cdot \mathrm{HBPh}_{4}\left(\mathrm{CDCl}_{3}, 298 \mathrm{~K}\right)$.

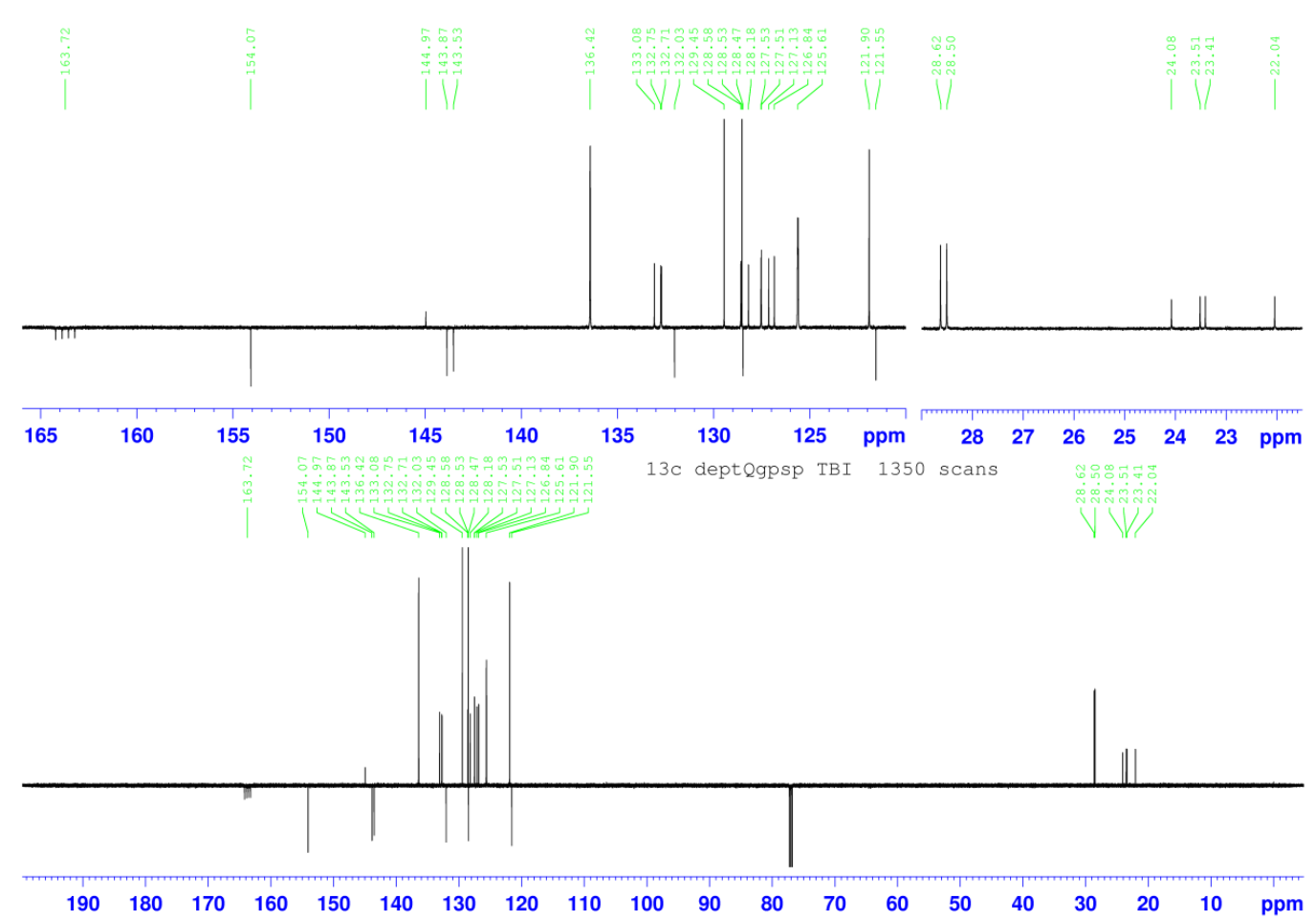

Figure $\mathrm{S} 51{ }^{13} \mathrm{C}(150 \mathrm{MHz})$ DEPTQ NMR spectrum of $21 \mathrm{c} \cdot \mathrm{HBPh}_{4}\left(\mathrm{CDCl}_{3}, 298 \mathrm{~K}\right)$. 


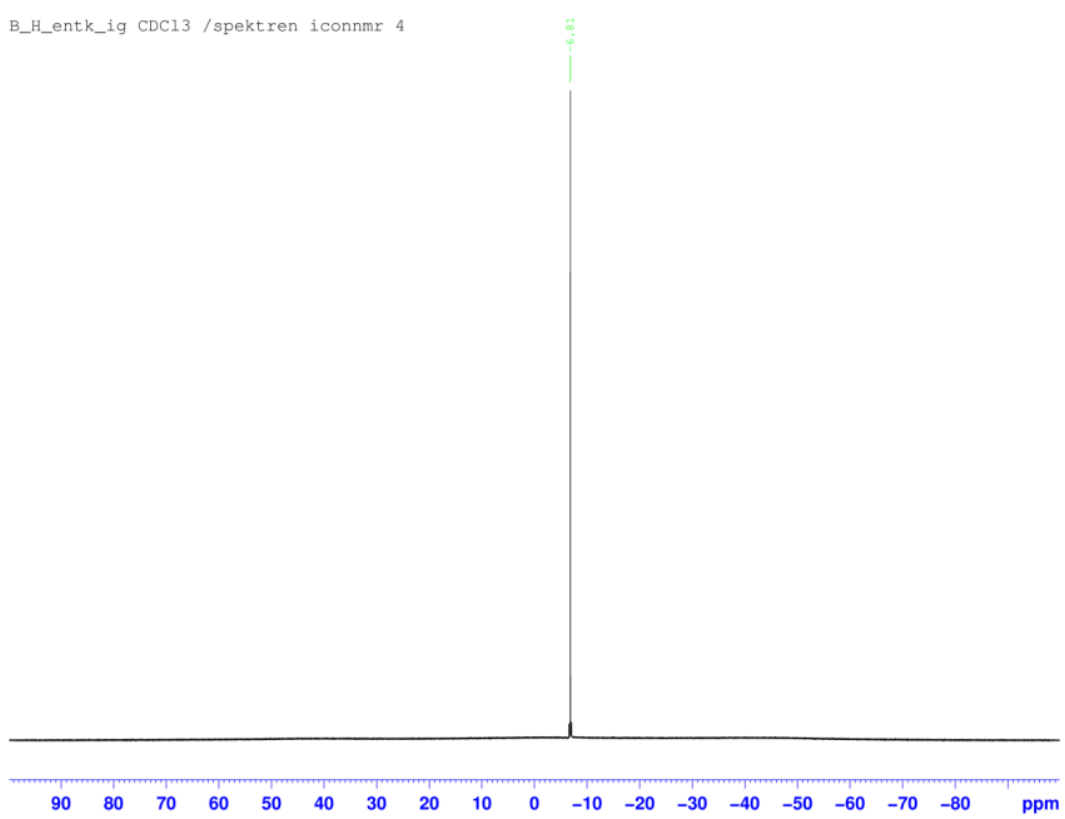

Figure S52 ${ }^{11} \mathrm{~B}(96 \mathrm{MHz}) \mathrm{NMR}$ spectrum of $21 \mathrm{c} \cdot \mathrm{HBPh}_{4}\left(\mathrm{CDCl}_{3}, 298 \mathrm{~K}\right)$.

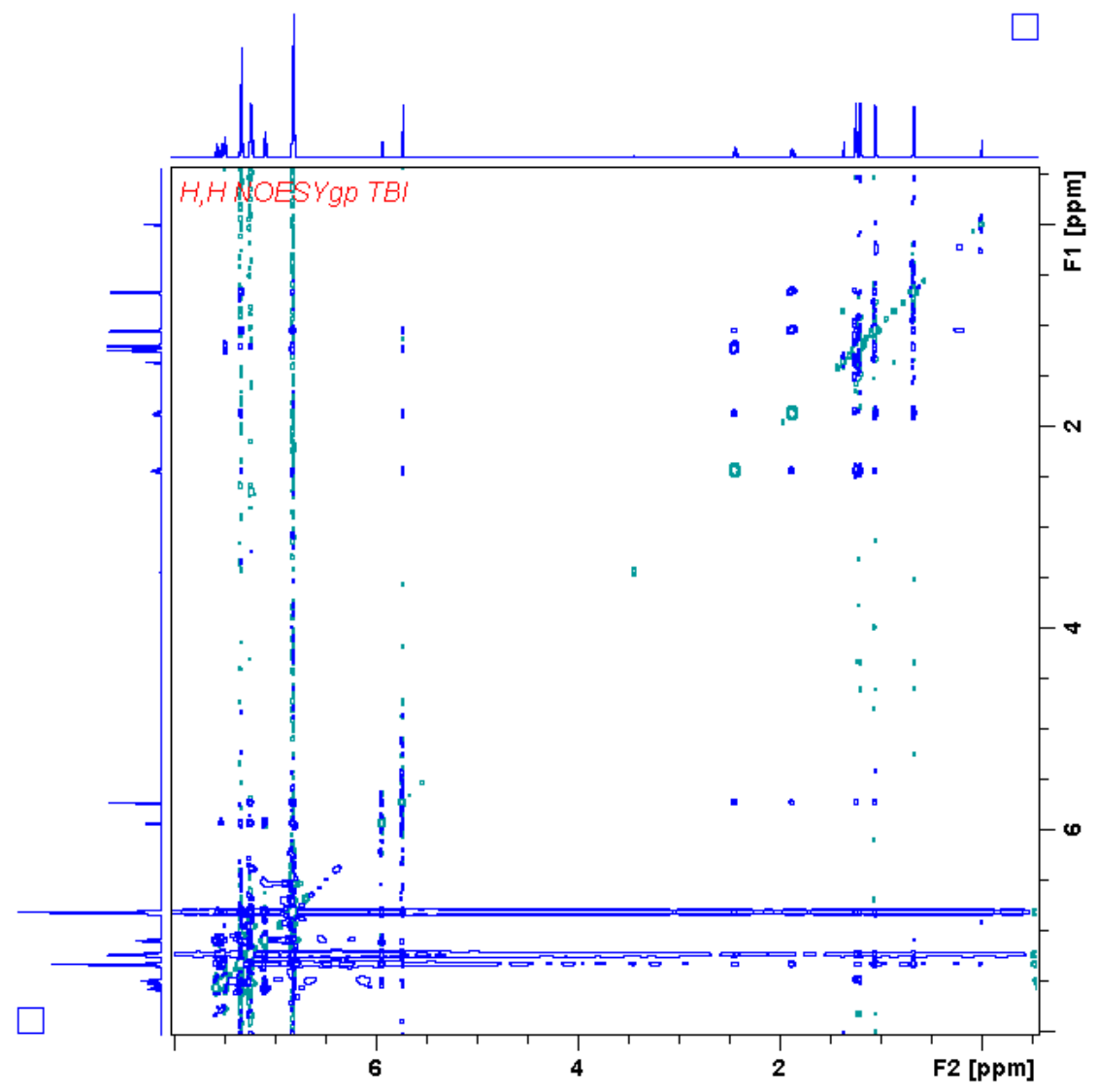

Figure S53 ${ }^{1} \mathrm{H},{ }^{1} \mathrm{H}$ NOESY NMR spectrum of $21 \mathrm{c} \cdot \mathrm{HBPh}_{4}\left(\mathrm{CDCl}_{3}, 600 \mathrm{MHz}, 298 \mathrm{~K}\right.$, mixing time $=600 \mathrm{~ms}$ ). 


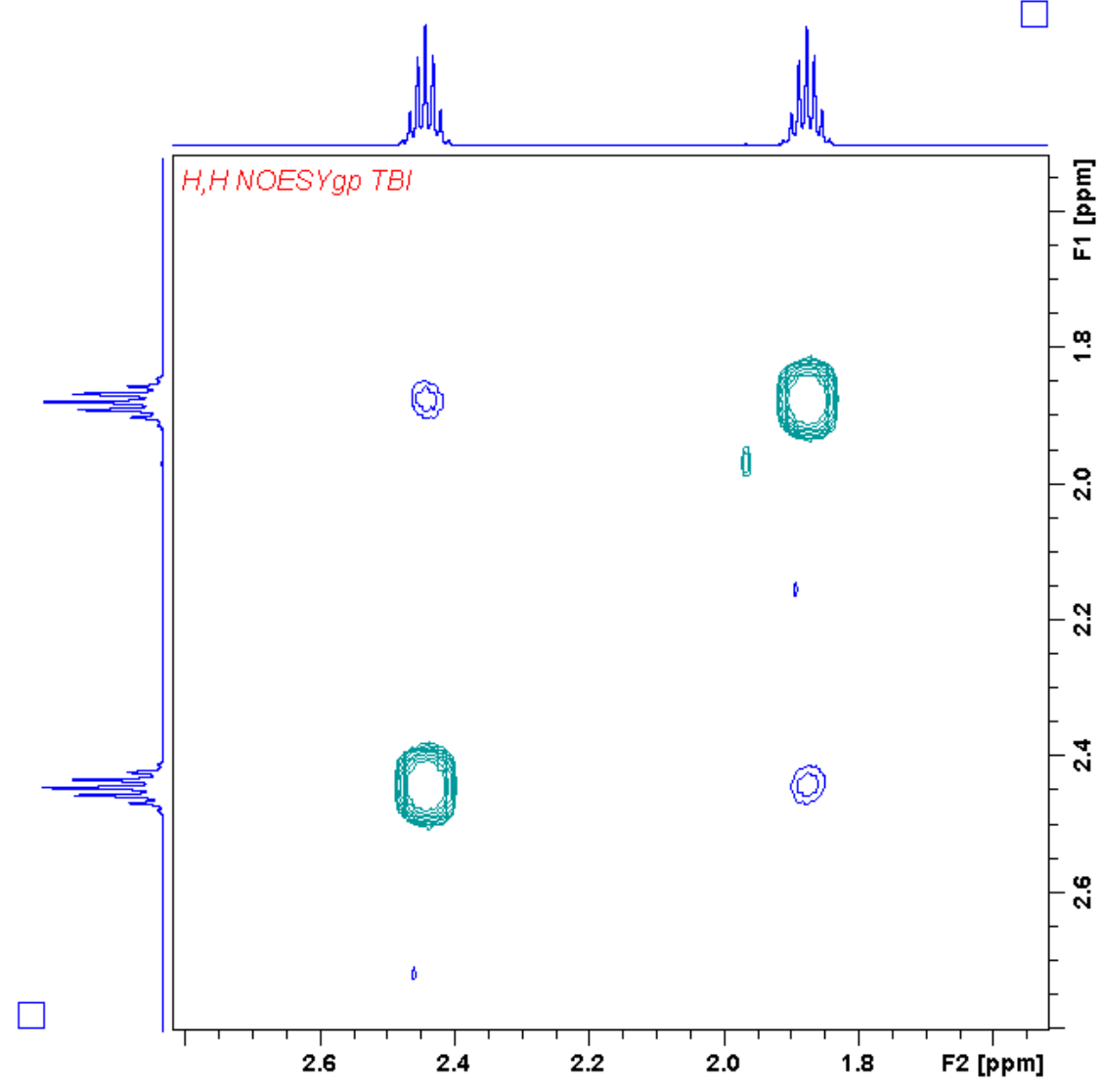

Figure S54 Part of ${ }^{1} \mathrm{H},{ }^{1} \mathrm{H}$ NOESY NMR spectrum of $21 \mathrm{c} \cdot \mathrm{HBPh}_{4}\left(\mathrm{CDCl}_{3}, 600 \mathrm{MHz}, 298 \mathrm{~K}\right.$, mixing time $=600 \mathrm{~ms}$ ).

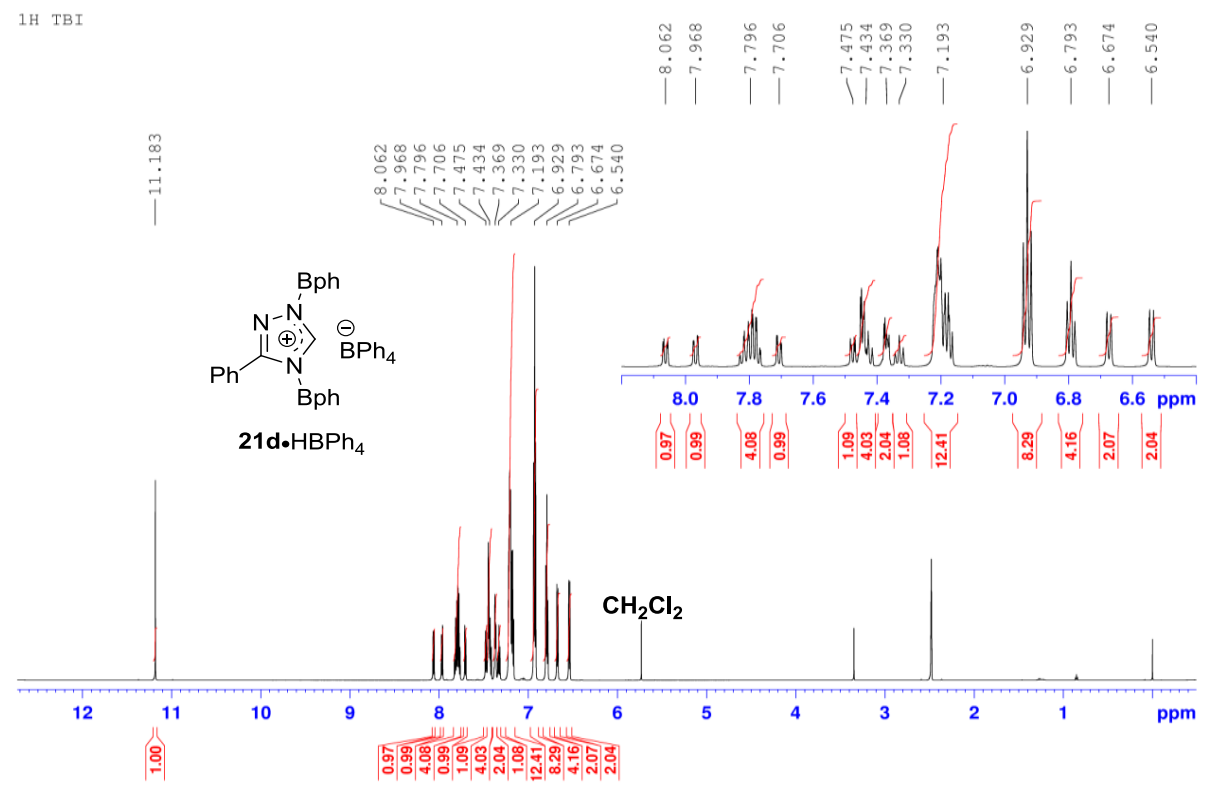

Figure $S 55{ }^{1} \mathrm{H}(600 \mathrm{MHz}) \mathrm{NMR}$ spectrum of $21 \mathrm{~d} \cdot \mathrm{HBPh}_{4}\left(\mathrm{DMSO}-d_{6}, 298 \mathrm{~K}\right)$. 


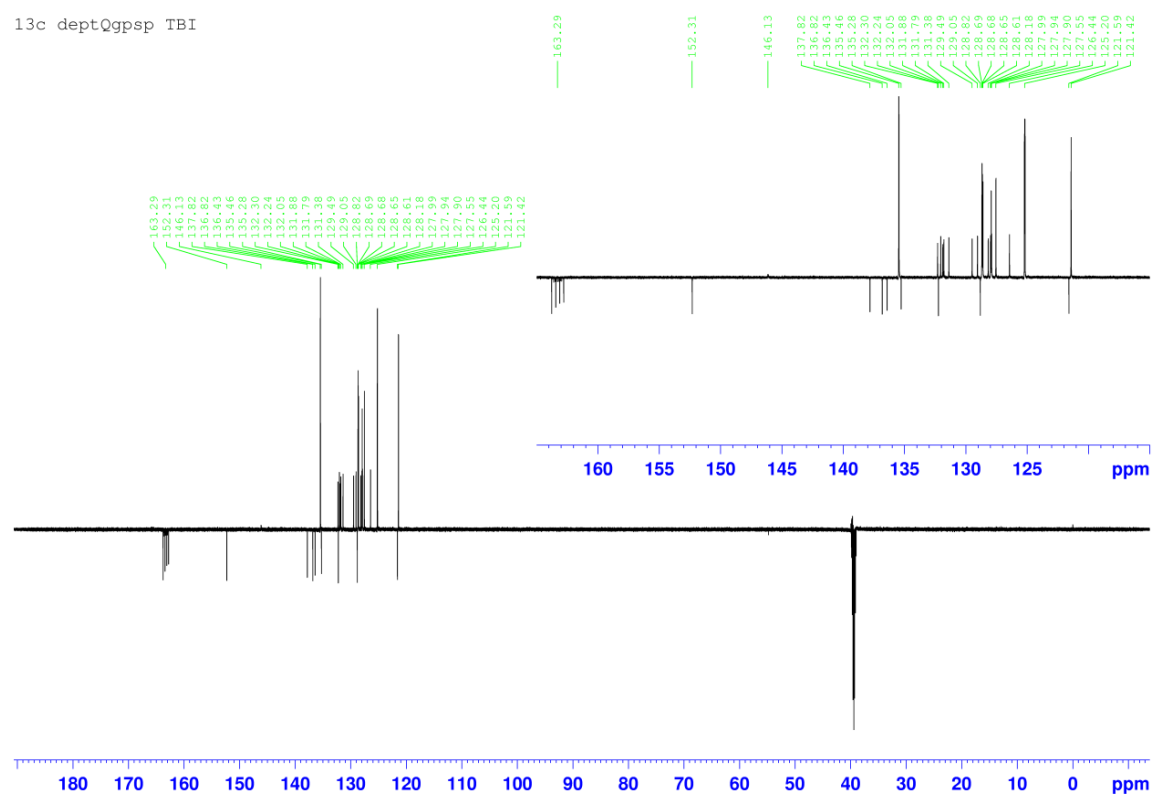

Figure $S 56{ }^{13} \mathrm{C}(150 \mathrm{MHz})$ DEPTQ NMR spectrum of 21d•HBPh $\left(\mathrm{DMSO}^{-} d_{6}, 298 \mathrm{~K}\right)$.

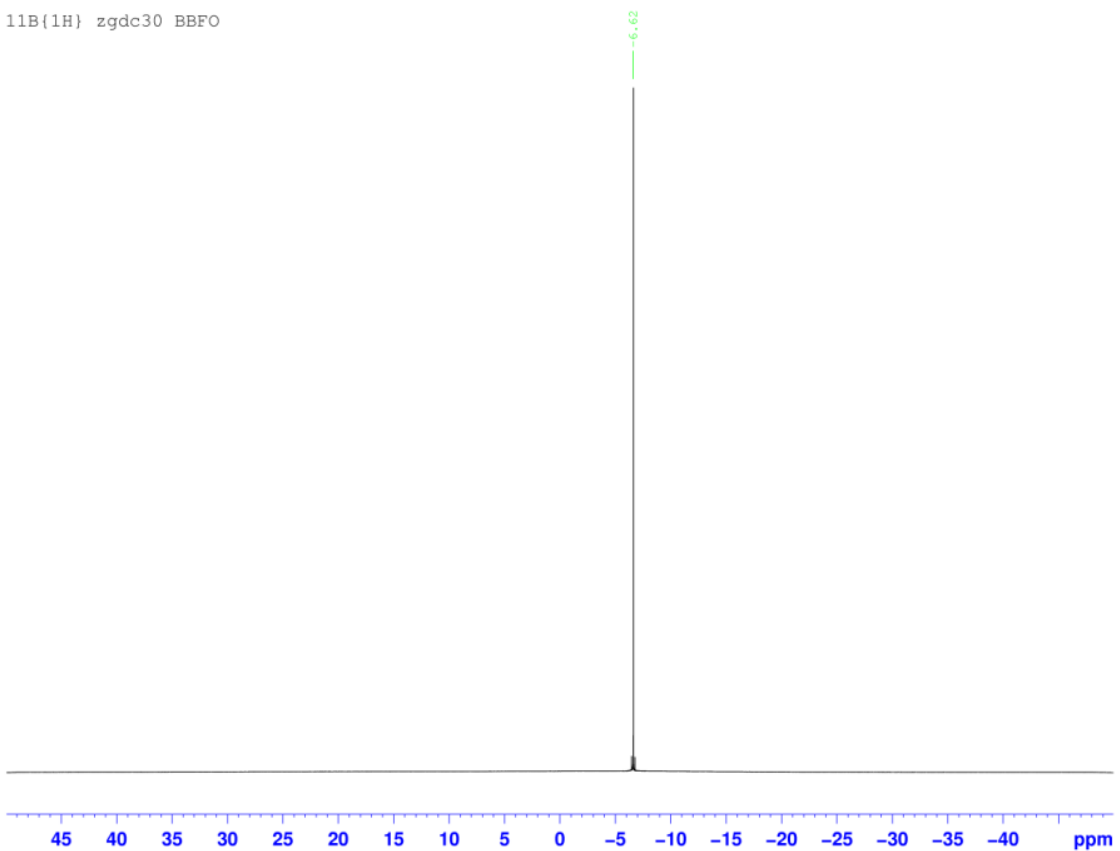

Figure $S 57^{11} \mathrm{~B}(160 \mathrm{MHz}) \mathrm{NMR}$ spectrum of $21 \mathrm{~d} \bullet \mathrm{HBPh}_{4}\left(\mathrm{DMSO}-\mathrm{d}_{6}, 298 \mathrm{~K}\right)$. 


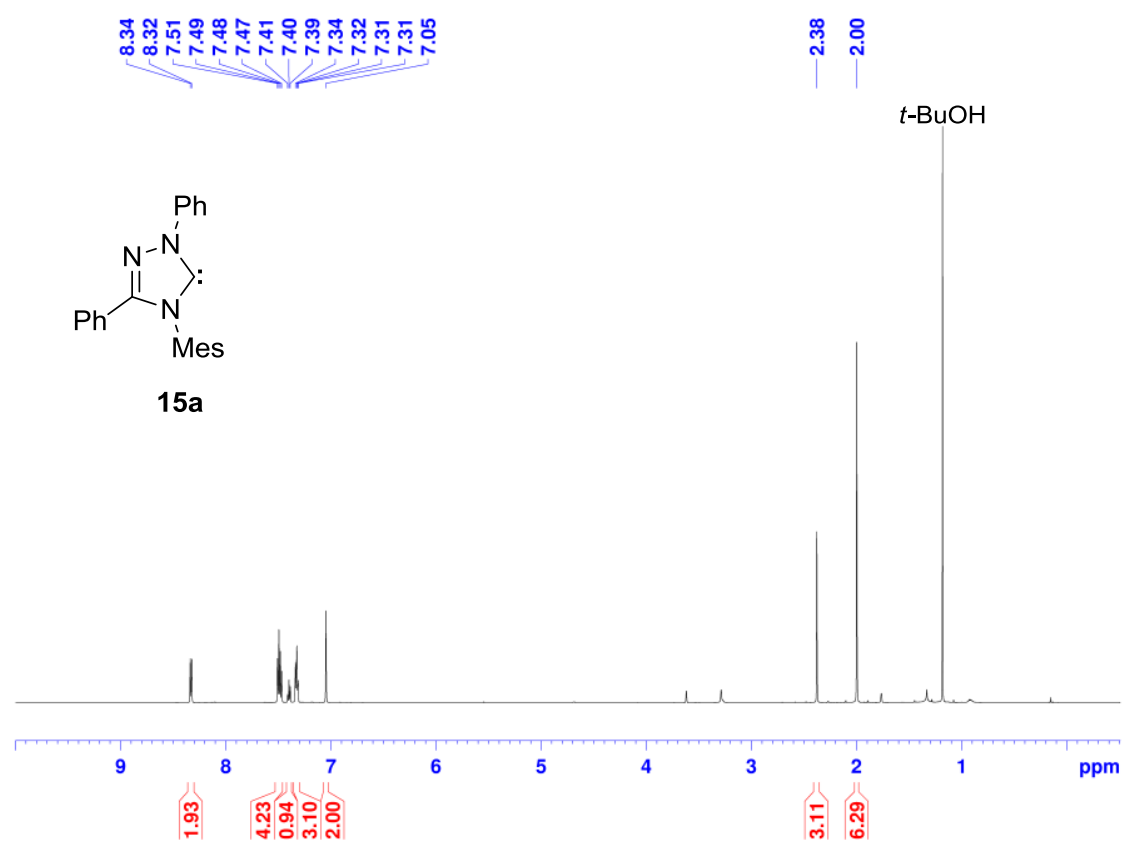

Figure $S 58{ }^{1} \mathrm{H}(600 \mathrm{MHz})$ NMR spectrum of $15 \mathrm{a}\left(\mathrm{THF}-d_{8}, 298 \mathrm{~K}\right)$.

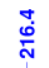

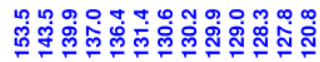

$\hat{\bar{i}}$ ( - -

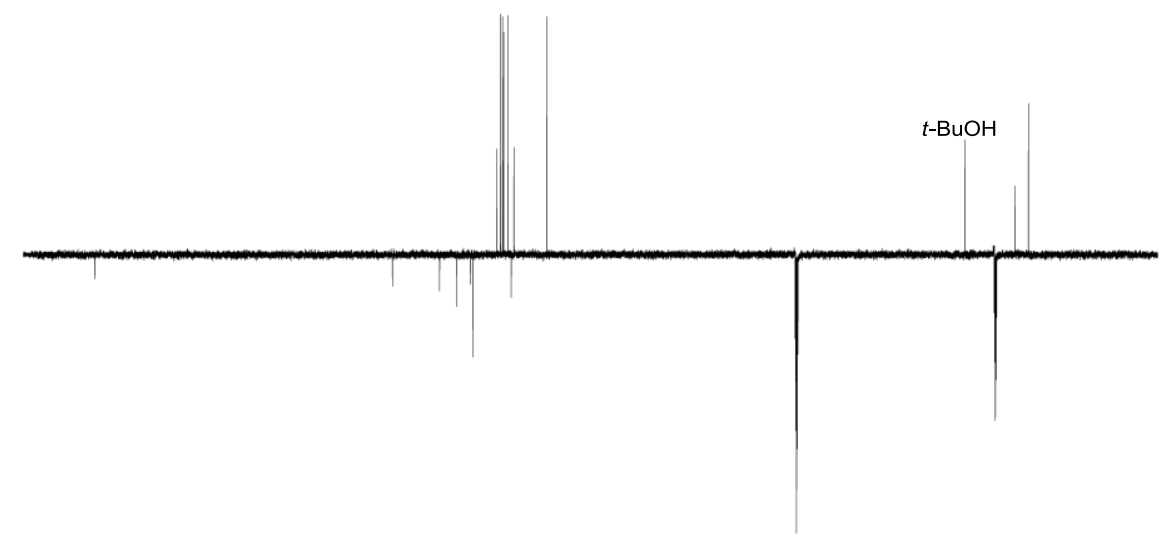

$\begin{array}{llllllllllll}220 & 200 & 180 & 160 & 140 & 120 & 100 & 80 & 60 & 40 & 20 & \mathrm{ppm}\end{array}$

Figure $559{ }^{13} \mathrm{C}(150 \mathrm{MHz})$ DEPTQ NMR spectrum of 15a (THF-d, $\left.298 \mathrm{~K}\right)$. 


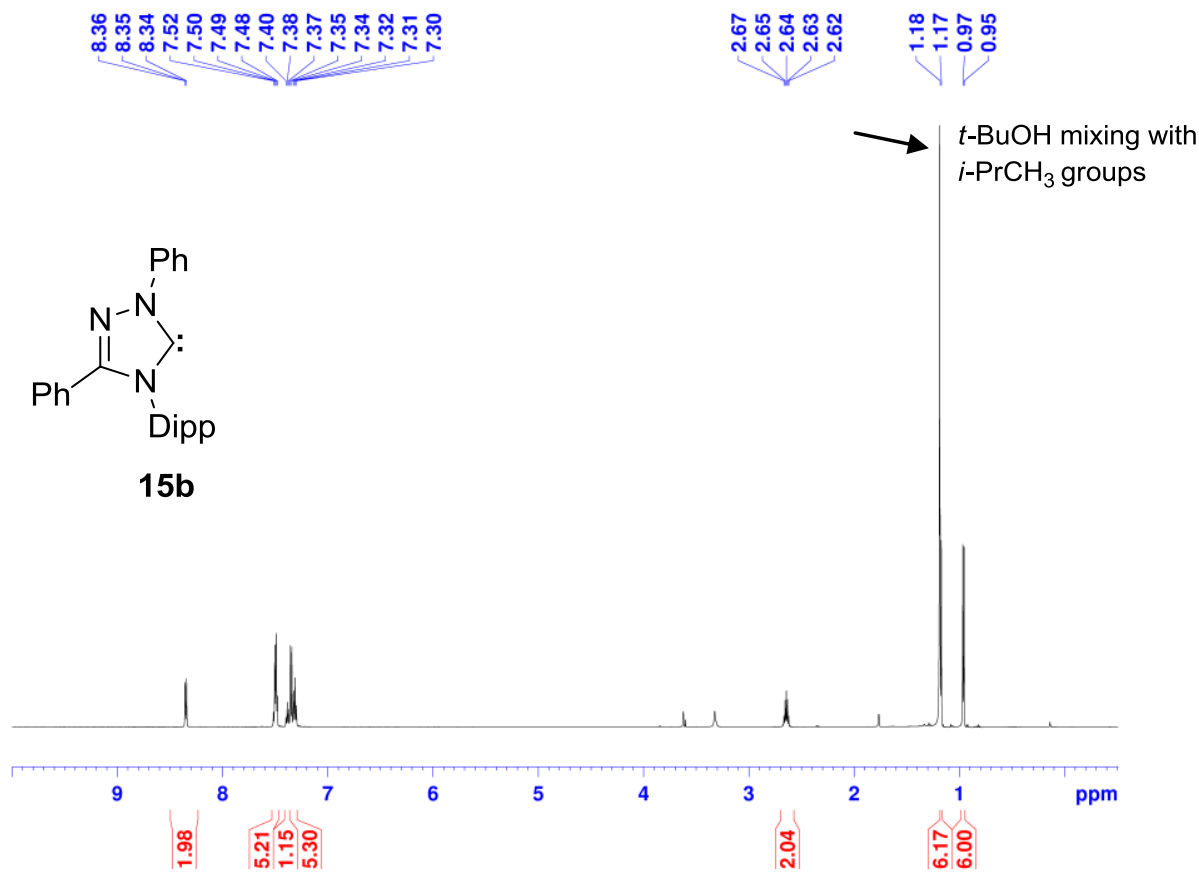

Figure $S 60^{1} \mathrm{H}(600 \mathrm{MHz}) \mathrm{NMR}$ spectrum of $15 \mathbf{b}\left(\mathrm{THF}-d_{8}, 298 \mathrm{~K}\right)$.

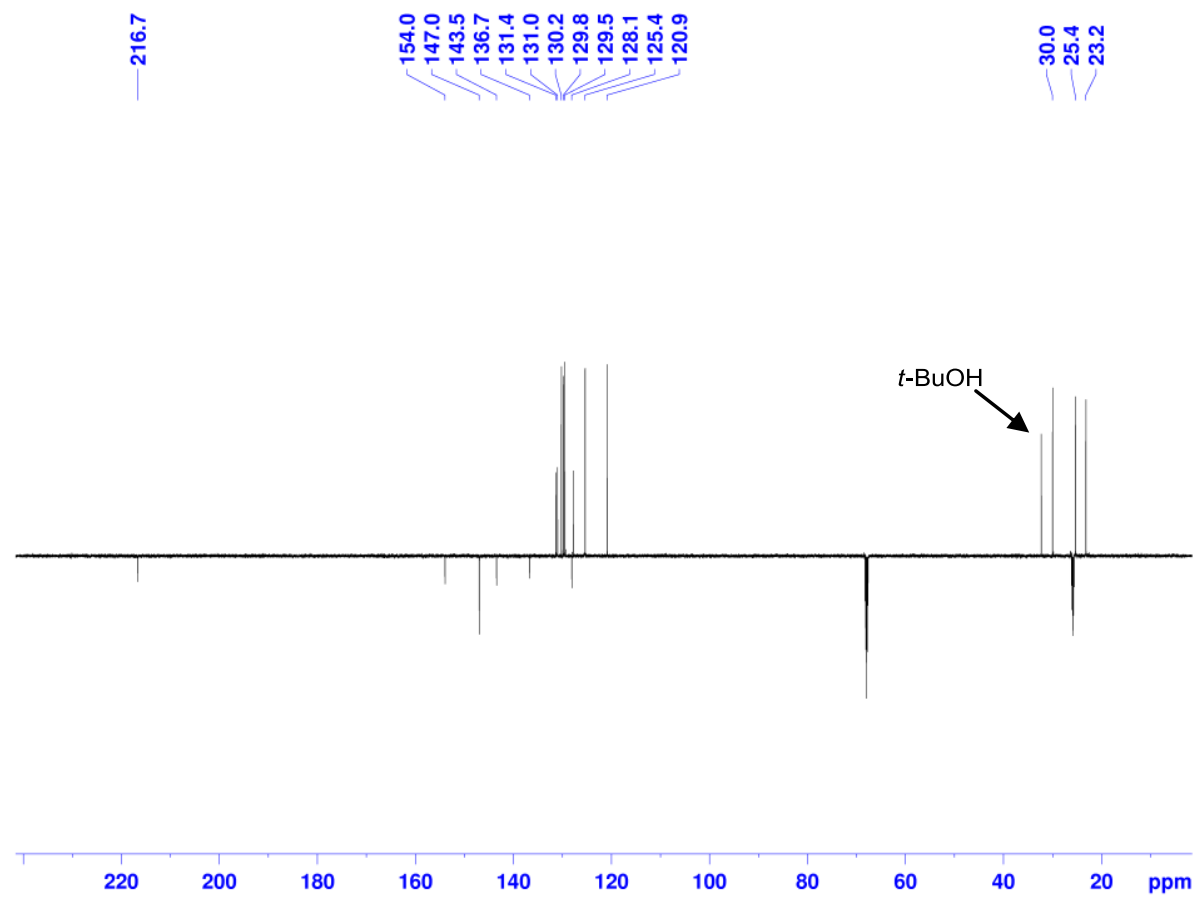

Figure $S 61{ }^{13} \mathrm{C}(150 \mathrm{MHz})$ DEPTQ NMR spectrum of 15b (THF-d, $\left.298 \mathrm{~K}\right)$. 


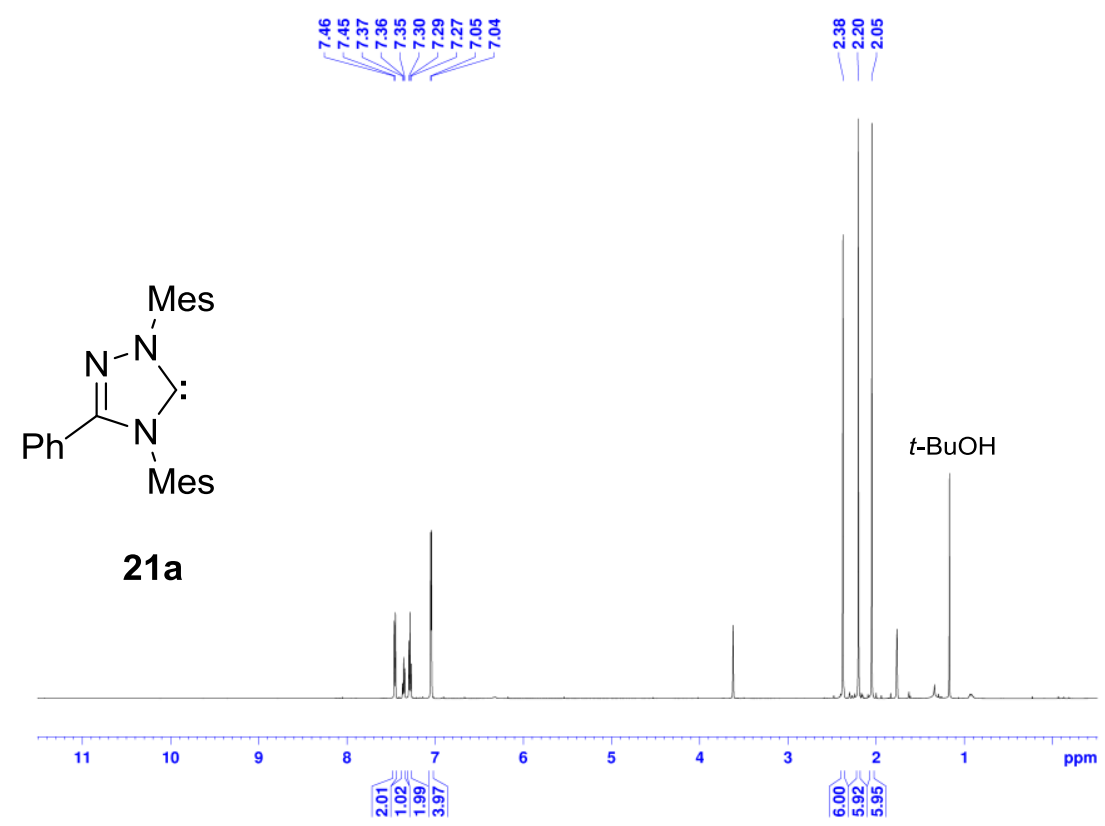

Figure $S 62{ }^{1} \mathrm{H}(600 \mathrm{MHz}) \mathrm{NMR}$ spectrum of 21a $\left(\mathrm{THF}-d_{8}, 298 \mathrm{~K}\right)$.

$$
\stackrel{\substack{\infty \\ \infty}}{\stackrel{\infty}{\pi}}
$$

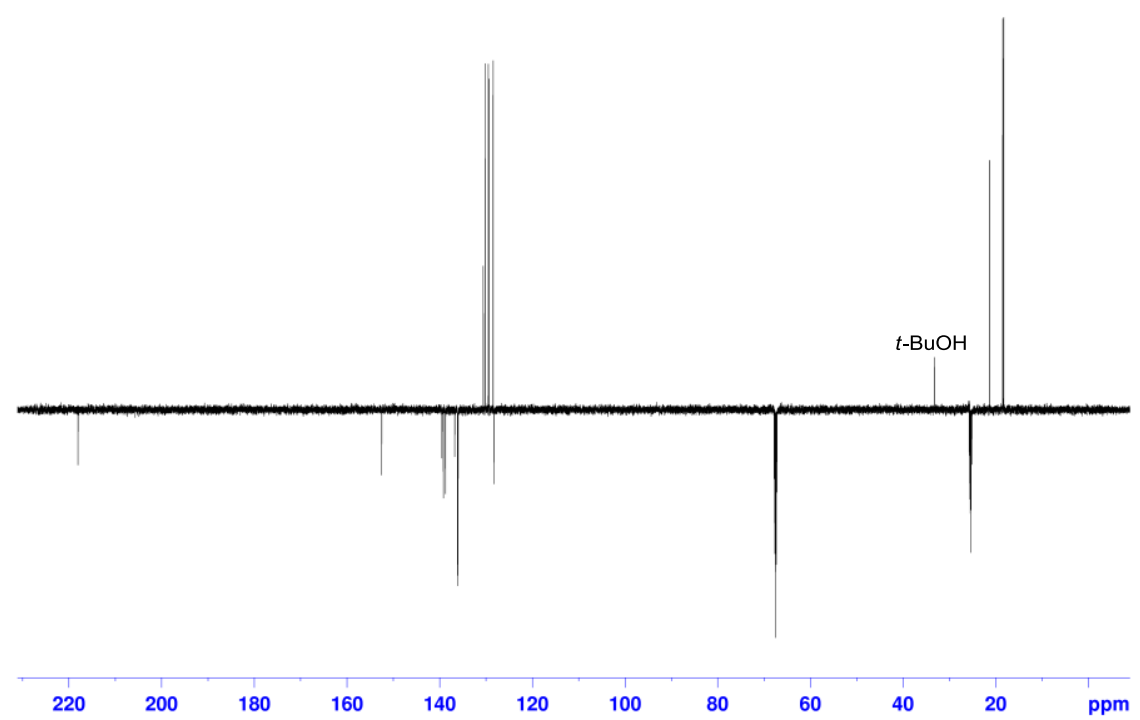

Figure $S 63{ }^{13} \mathrm{C}(150 \mathrm{MHz})$ DEPTQ NMR spectrum of 21a $\left(\mathrm{THF}-d_{8}, 298 \mathrm{~K}\right)$. 
<smiles>O=P(O)(c1ccccc1)N1CN(c2ccccc2)C(c2ccccc2)=N1</smiles>

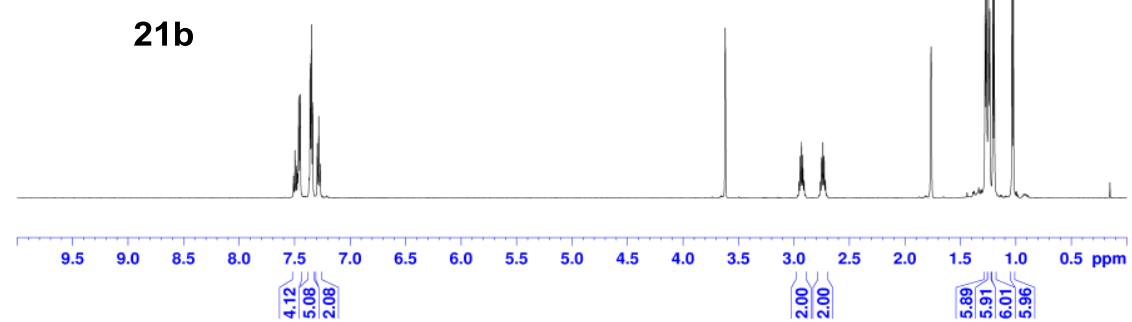

Figure $S 64{ }^{1} \mathrm{H}(600 \mathrm{MHz}) \mathrm{NMR}$ spectrum of 21b (THF-d $\left.d_{8}, 298 \mathrm{~K}\right)$.

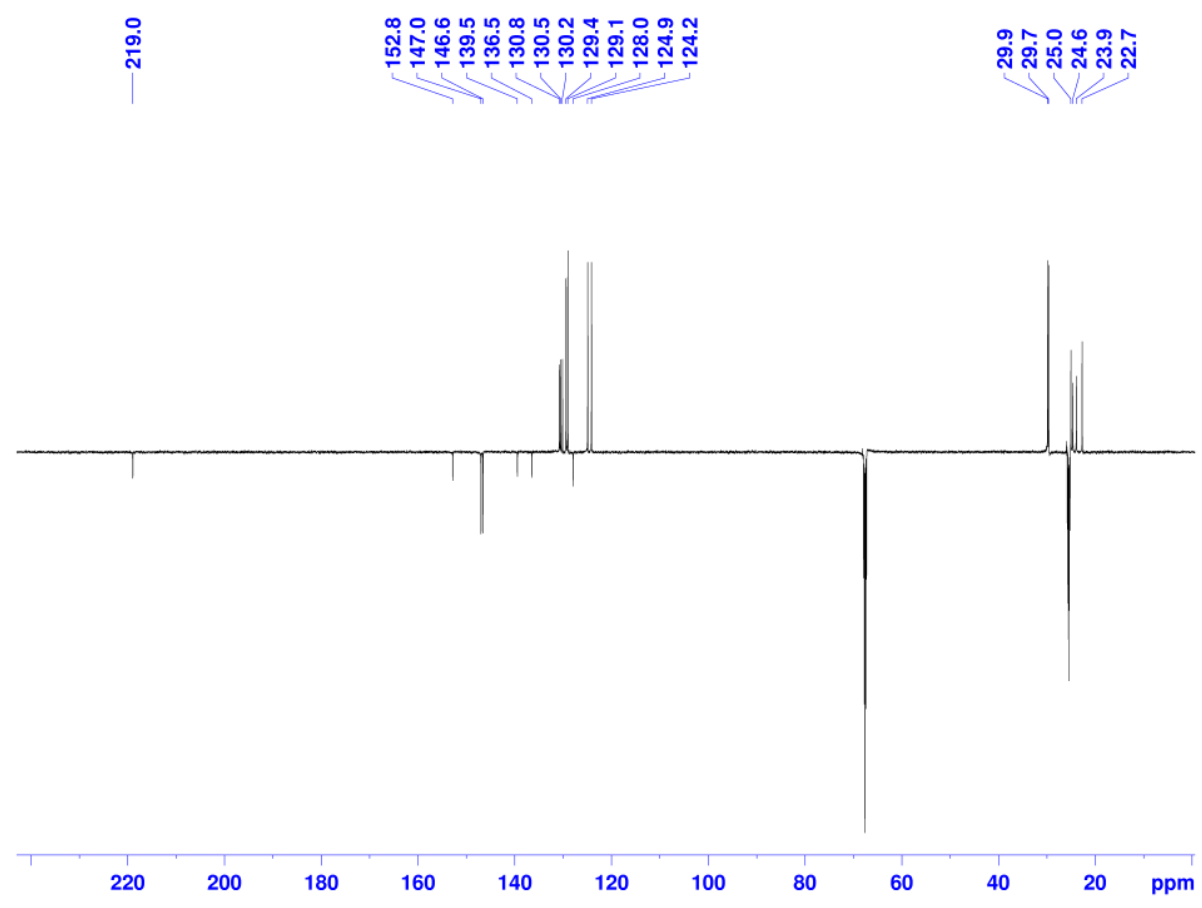

Figure $S 65{ }^{13} \mathrm{C}(150 \mathrm{MHz})$ DEPTQ NMR spectrum of $21 \mathrm{~b}\left(\mathrm{THF}-d_{8}, 298 \mathrm{~K}\right)$. 


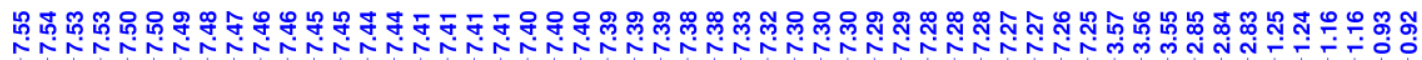

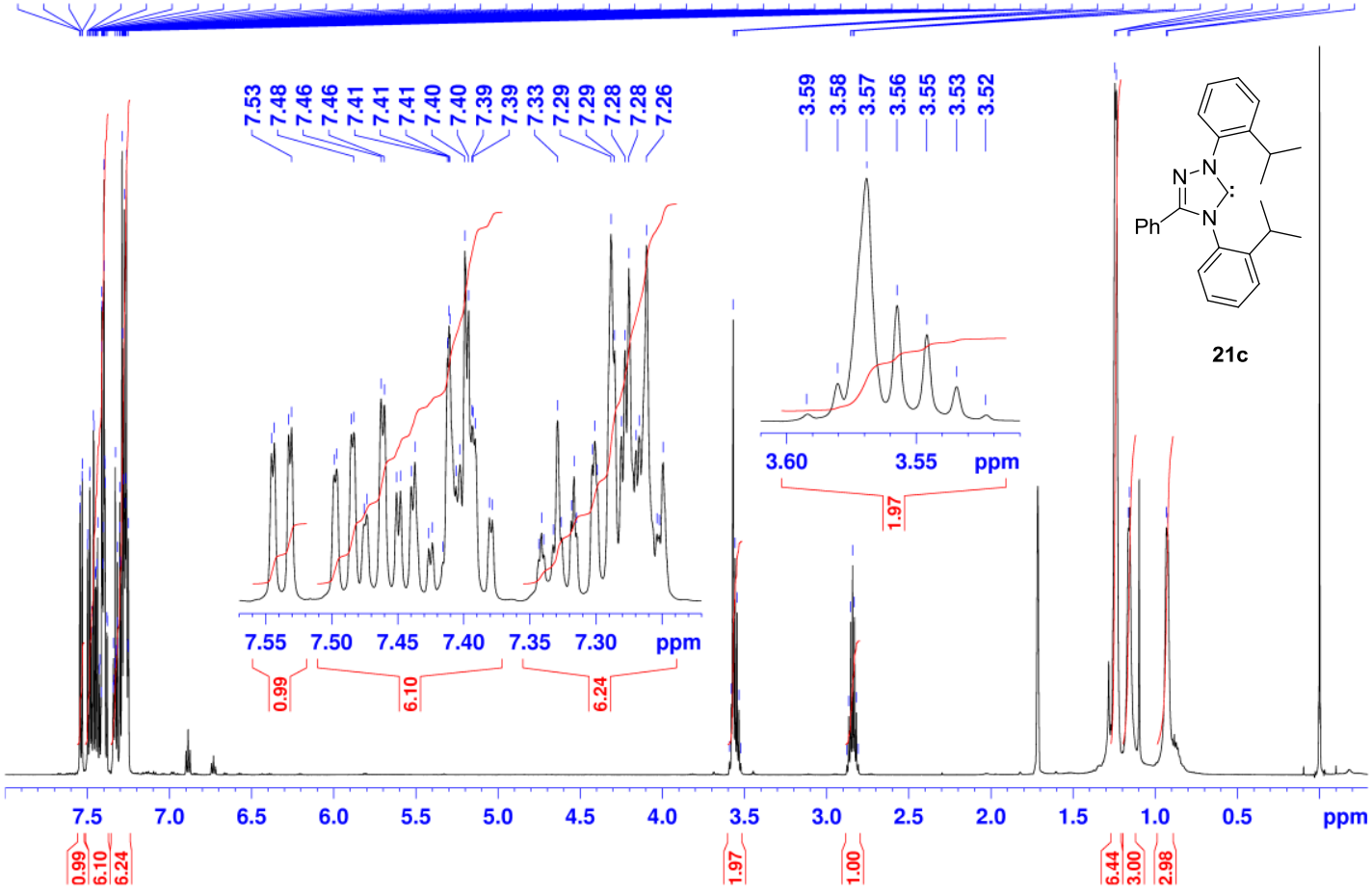

Figure $S 66{ }^{1} \mathrm{H}$ NMR spectrum $(600 \mathrm{MHz})$ of $21 \mathrm{c}\left(\mathrm{THF}^{-} \mathrm{d}_{8}, 298 \mathrm{~K}\right)$.

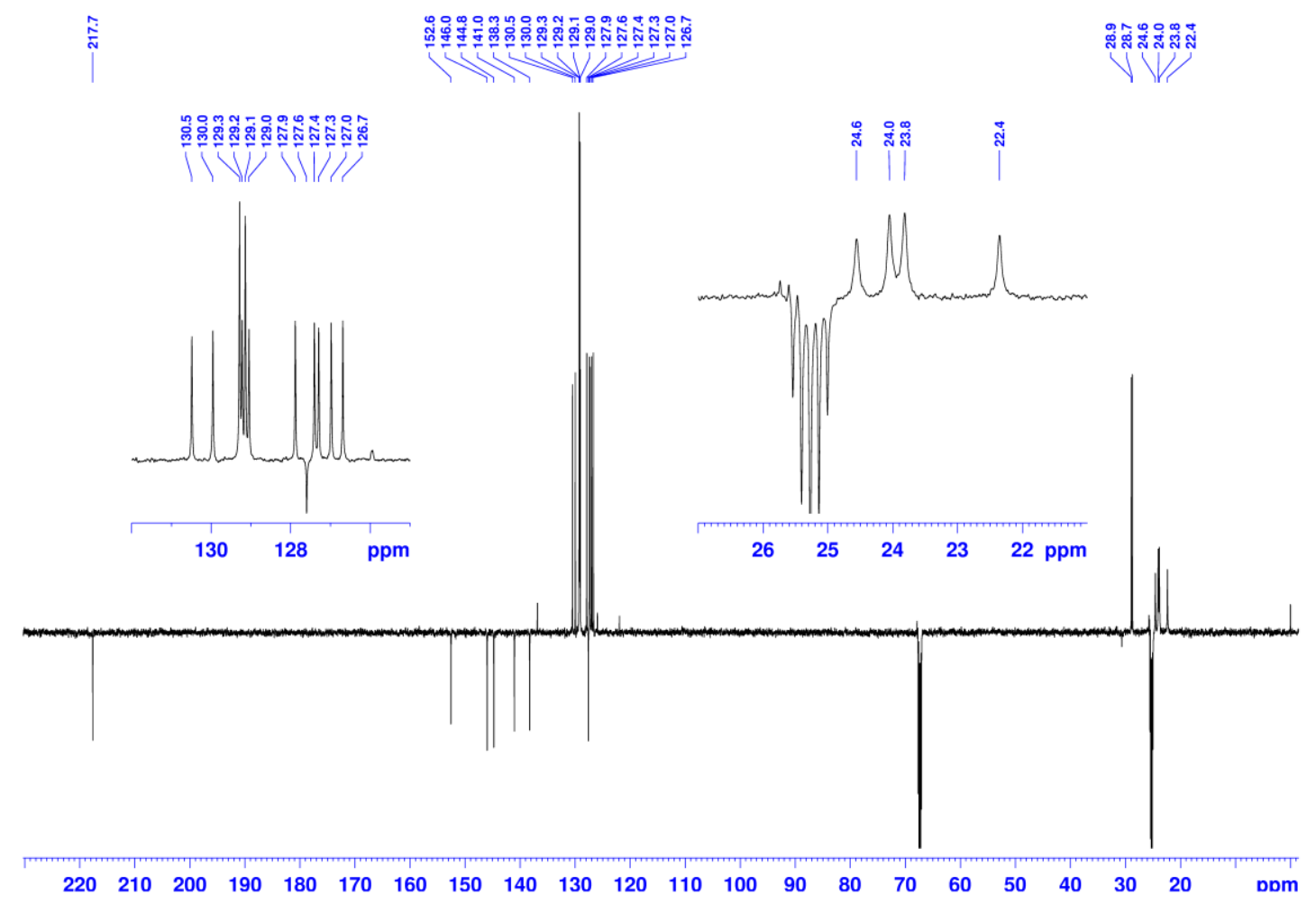

Figure $567{ }^{13} \mathrm{C}$ DEPTQ NMR spectrum $(150 \mathrm{MHz})$ of $21 \mathrm{c}\left(\mathrm{THF}-\mathrm{d}_{8}, 298 \mathrm{~K}\right)$. 


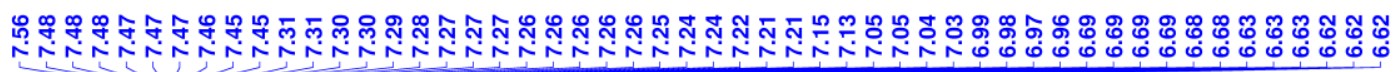

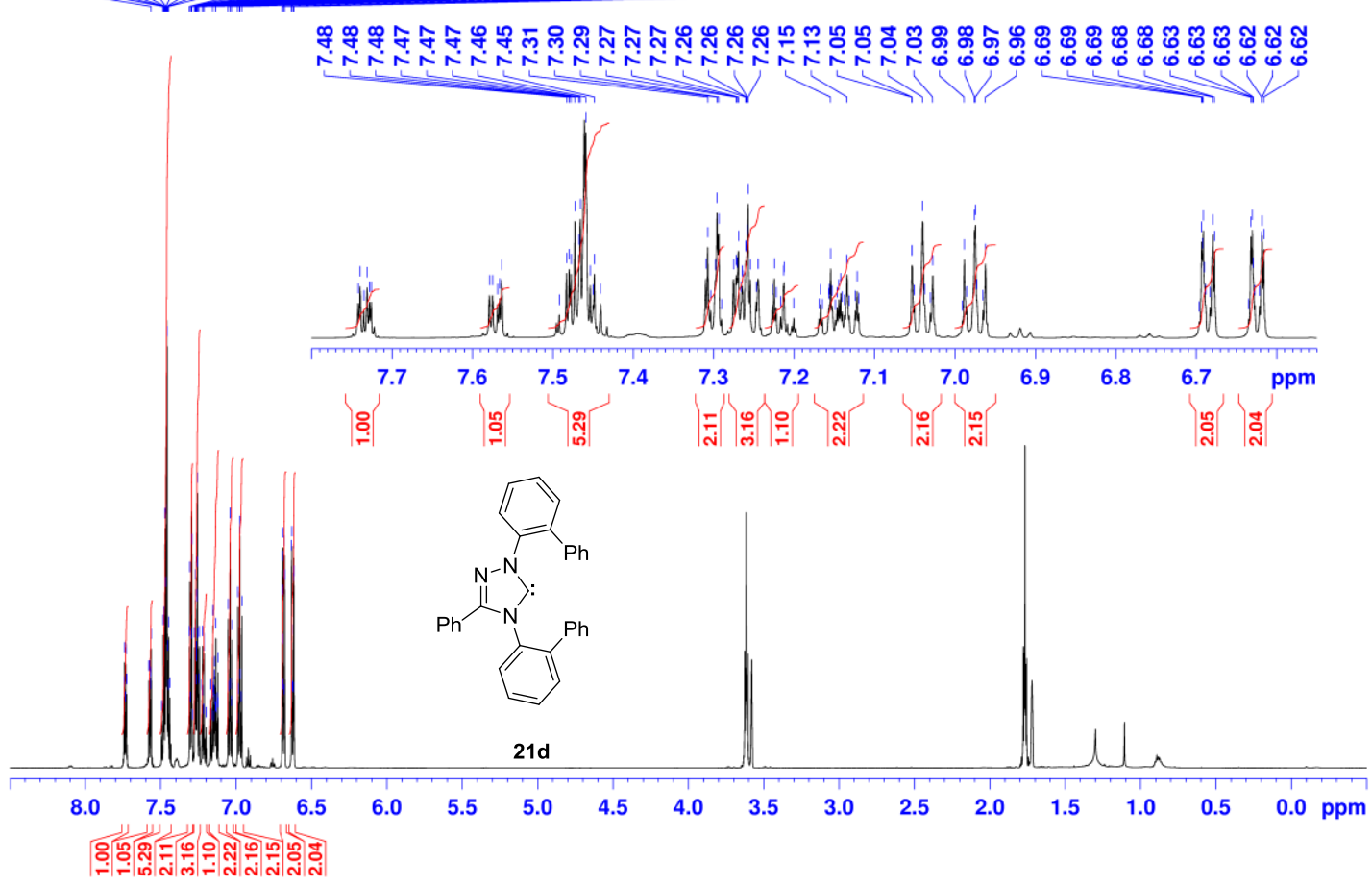

Figure S68 ${ }^{1} \mathrm{H}$ NMR spectrum $(600 \mathrm{MHz})$ of $21 \mathrm{~d}\left(\mathrm{THF}-\mathrm{d}_{8}, 298 \mathrm{~K}\right)$.

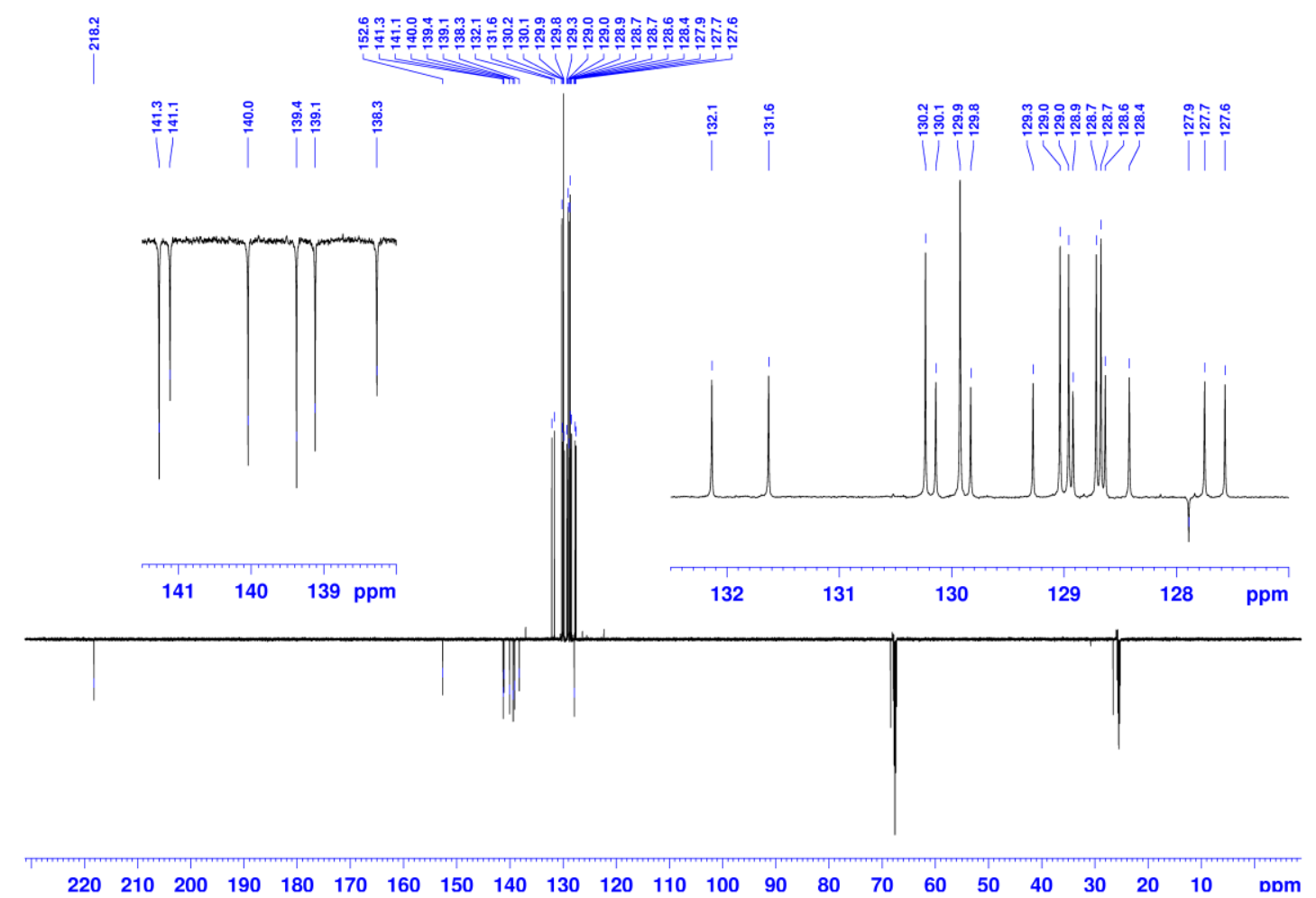

Figure $569{ }^{13} \mathrm{C}$ DEPTQ NMR spectrum $(150 \mathrm{MHz})$ of $21 \mathrm{~d}\left(\mathrm{THF}-d_{8}, 298 \mathrm{~K}\right)$. 


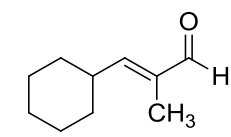

$25 a$

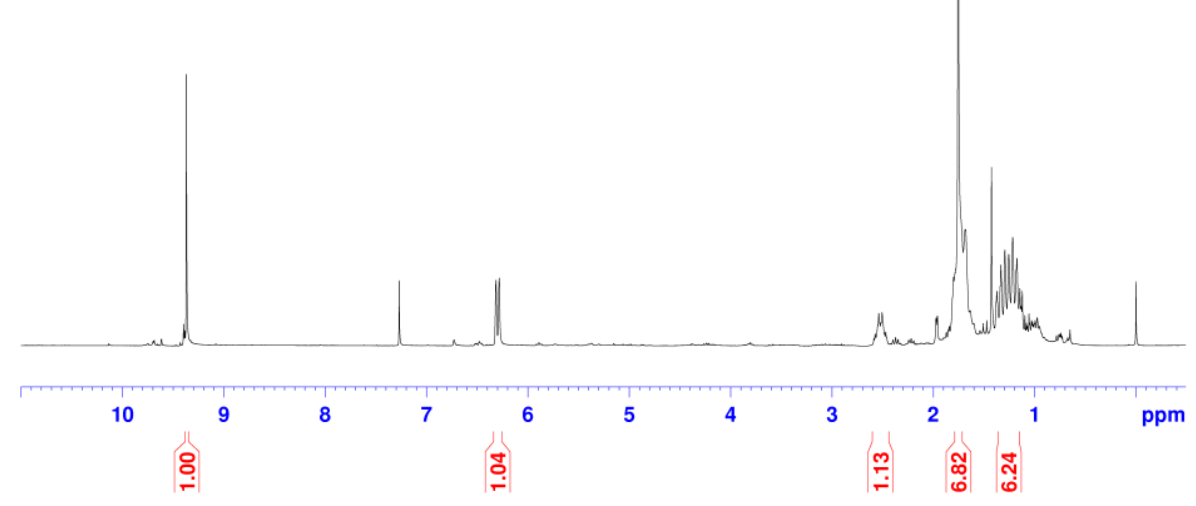

Figure $570^{1} \mathrm{H}(600 \mathrm{MHz}) \mathrm{NMR}$ spectrum of $25 \mathrm{a}\left(\mathrm{CDCl}_{3}, 298 \mathrm{~K}\right)$.
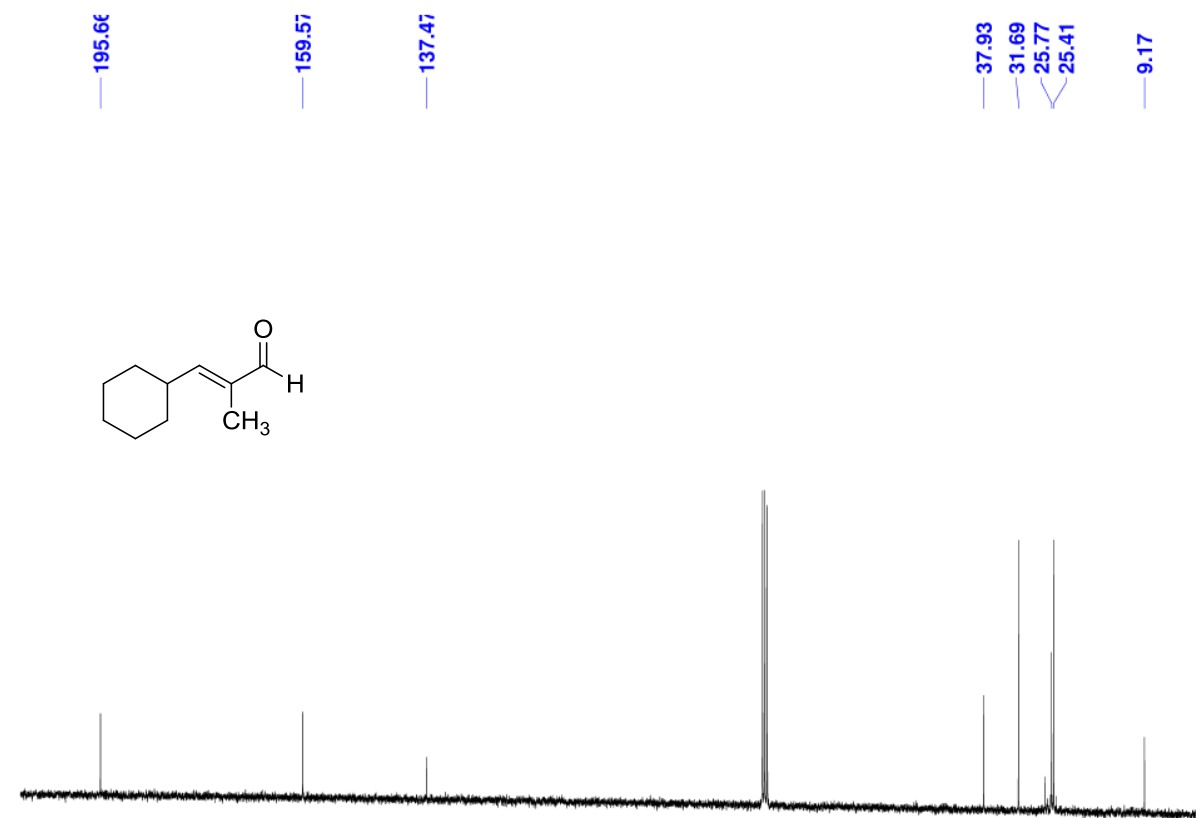

$\begin{array}{llllllllllllllllllll}200 & 190 & 180 & 170 & 160 & 150 & 140 & 130 & 120 & 110 & 100 & 90 & 80 & 70 & 60 & 50 & 40 & 30 & 20 & \mathrm{ppm}\end{array}$

Figure $S 71{ }^{13} \mathrm{C}(150 \mathrm{MHz}) \mathrm{NMR}$ spectrum of $25 \mathrm{a}\left(\mathrm{CDCl}_{3}, 298 \mathrm{~K}\right)$. 


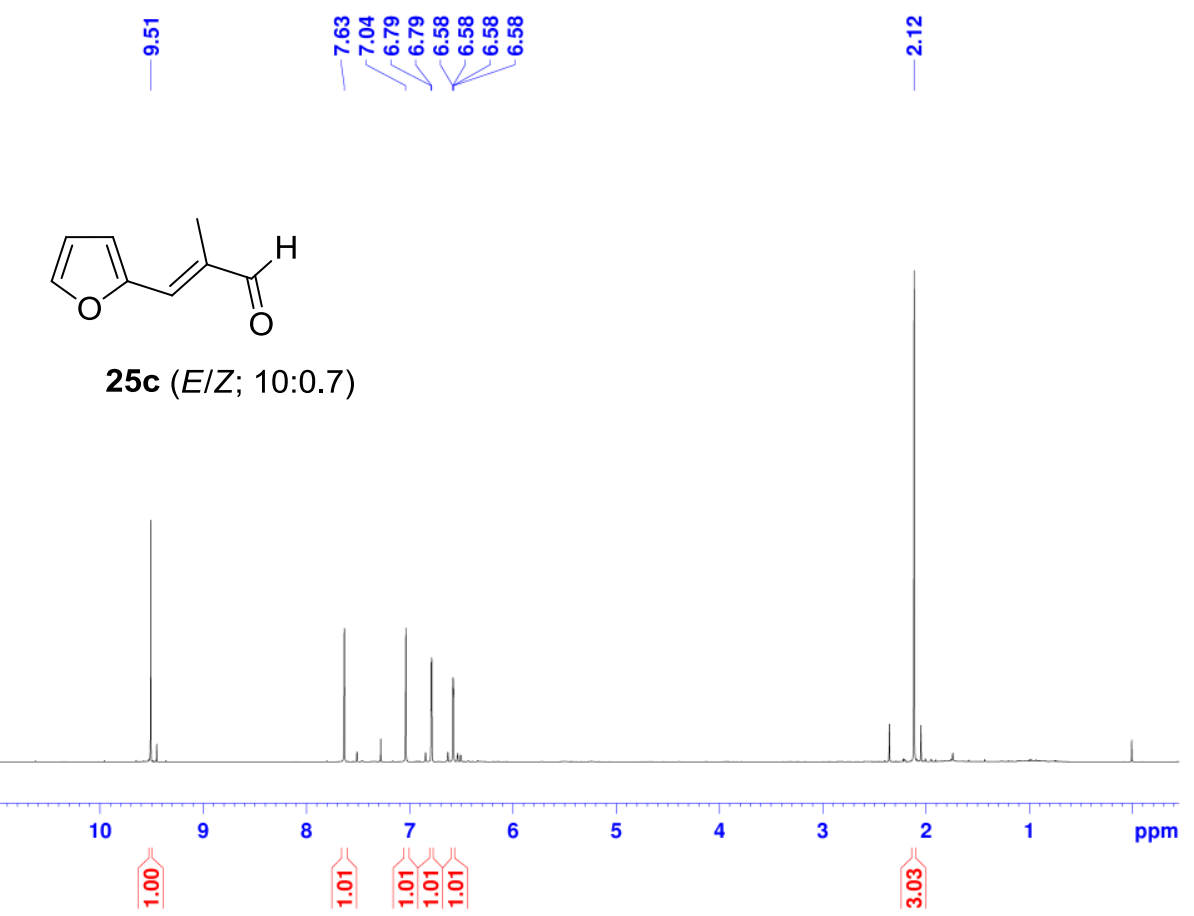

Figure $S 72{ }^{1} \mathrm{H}(600 \mathrm{MHz}) \mathrm{NMR}$ spectrum of $25 \mathrm{c}\left(\mathrm{CDCl}_{3}, 298 \mathrm{~K}\right)$.

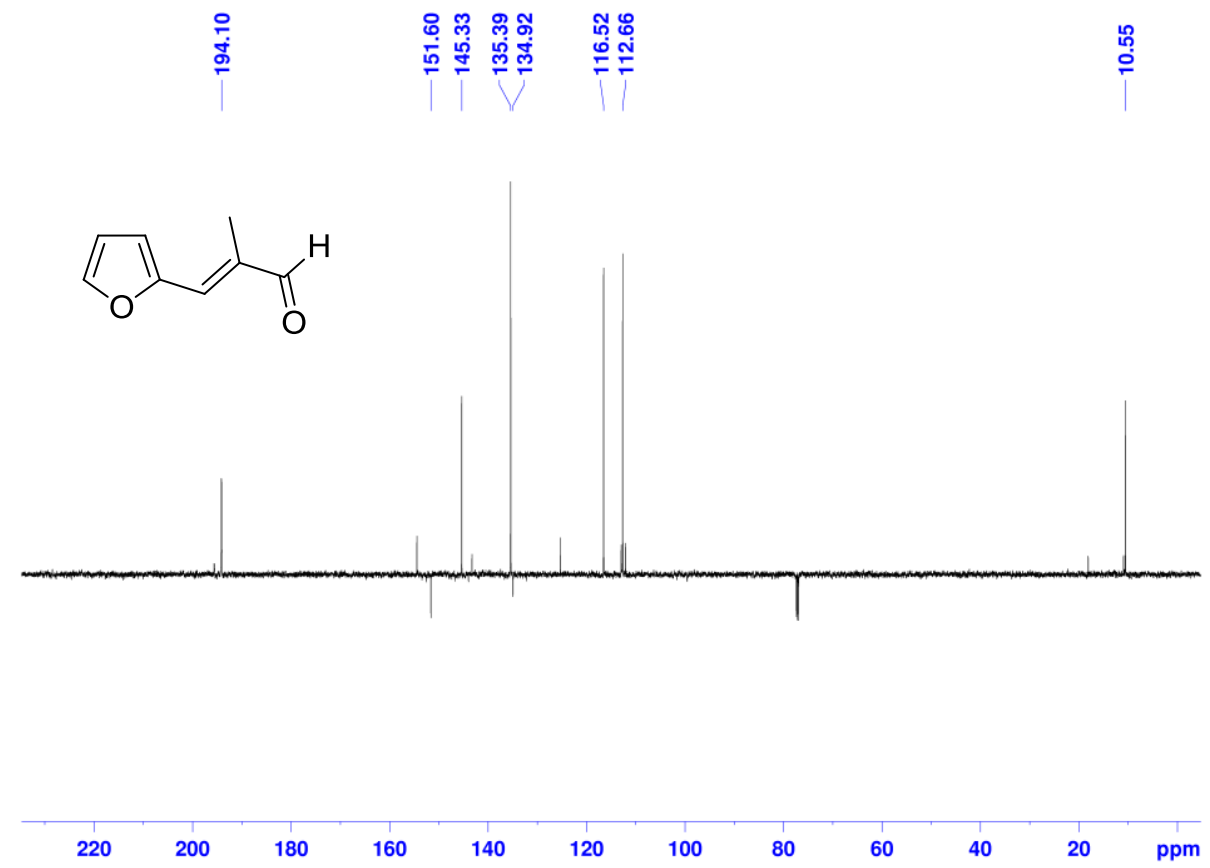

Figure $\mathbf{S 7 3}{ }^{13} \mathrm{C}(150 \mathrm{MHz})$ DEPTQ NMR spectrum of $25 \mathrm{c}\left(\mathrm{CDCl}_{3}, 298 \mathrm{~K}\right)$. 


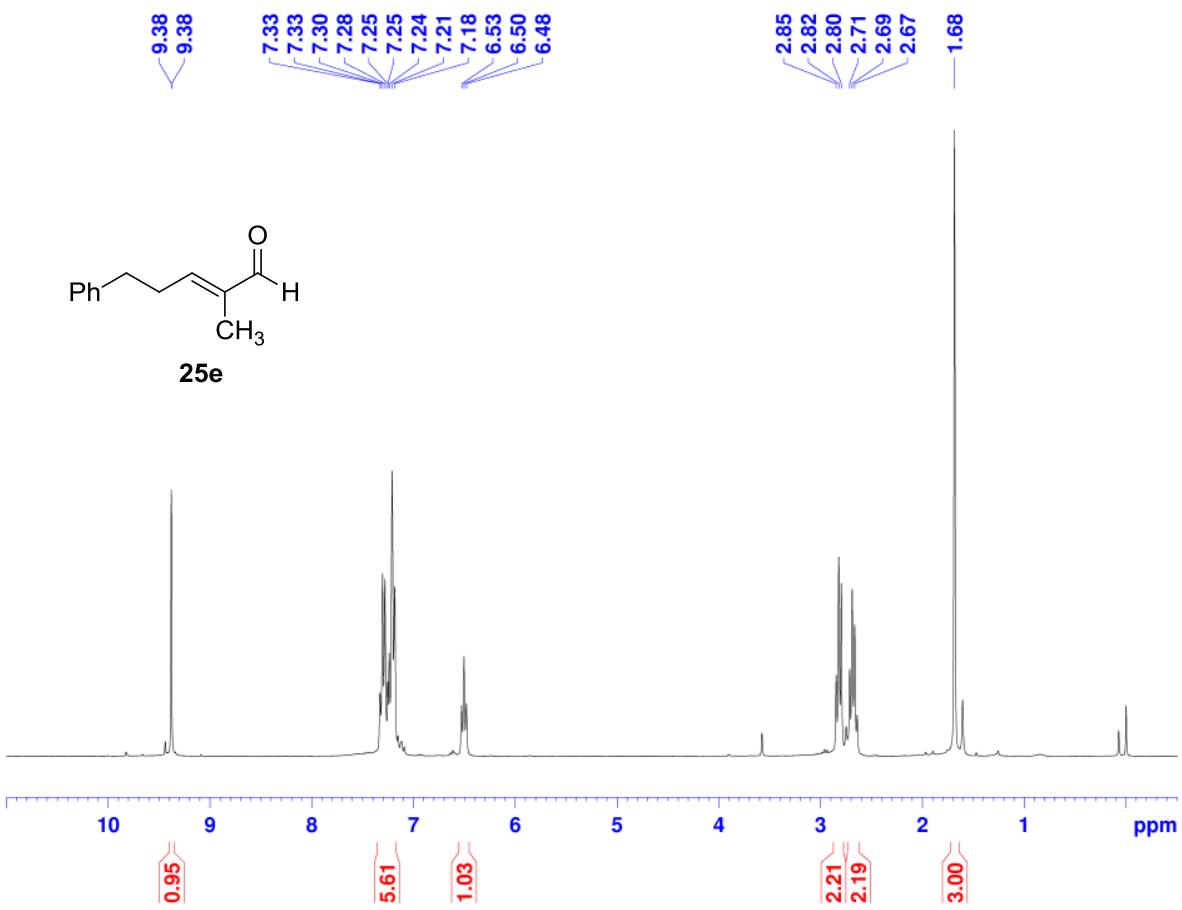

Figure $S 74{ }^{1} \mathrm{H}(600 \mathrm{MHz}) \mathrm{NMR}$ spectrum of $25 \mathrm{e}\left(\mathrm{CDCl}_{3}, 298 \mathrm{~K}\right)$.
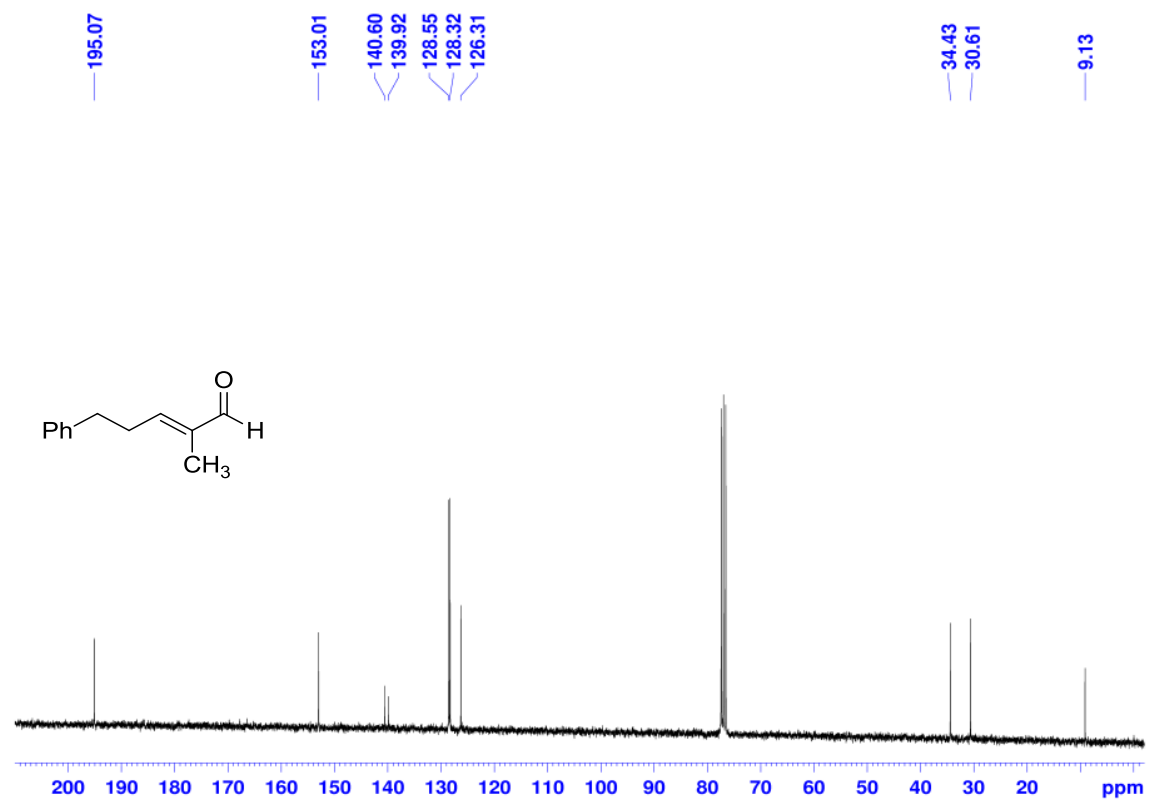

Figure $S 75{ }^{13} \mathrm{C}(150 \mathrm{MHz}) \mathrm{NMR}$ spectrum of $25 \mathrm{e}\left(\mathrm{CDCl}_{3}, 298 \mathrm{~K}\right)$. 


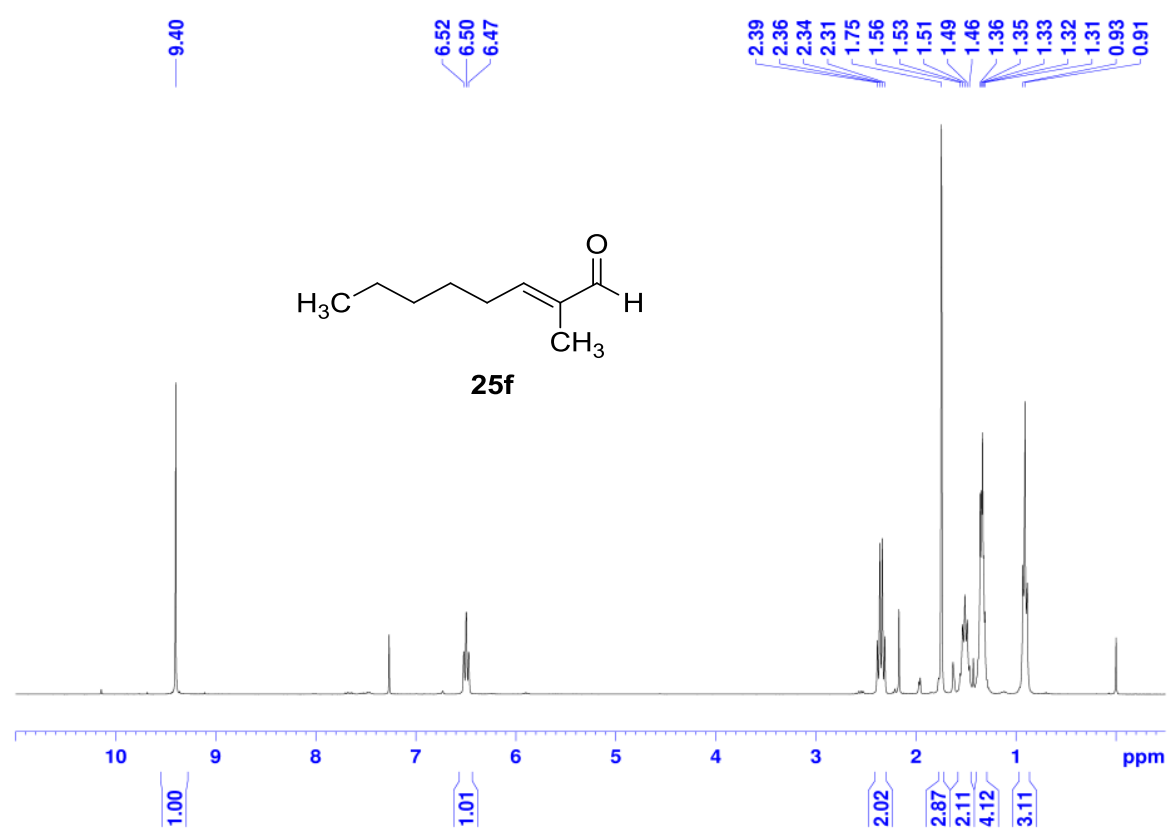

Figure $S 76{ }^{1} \mathrm{H}(600 \mathrm{MHz}) \mathrm{NMR}$ spectrum of $25 \mathrm{f}\left(\mathrm{CDCl}_{3}, 298 \mathrm{~K}\right)$.
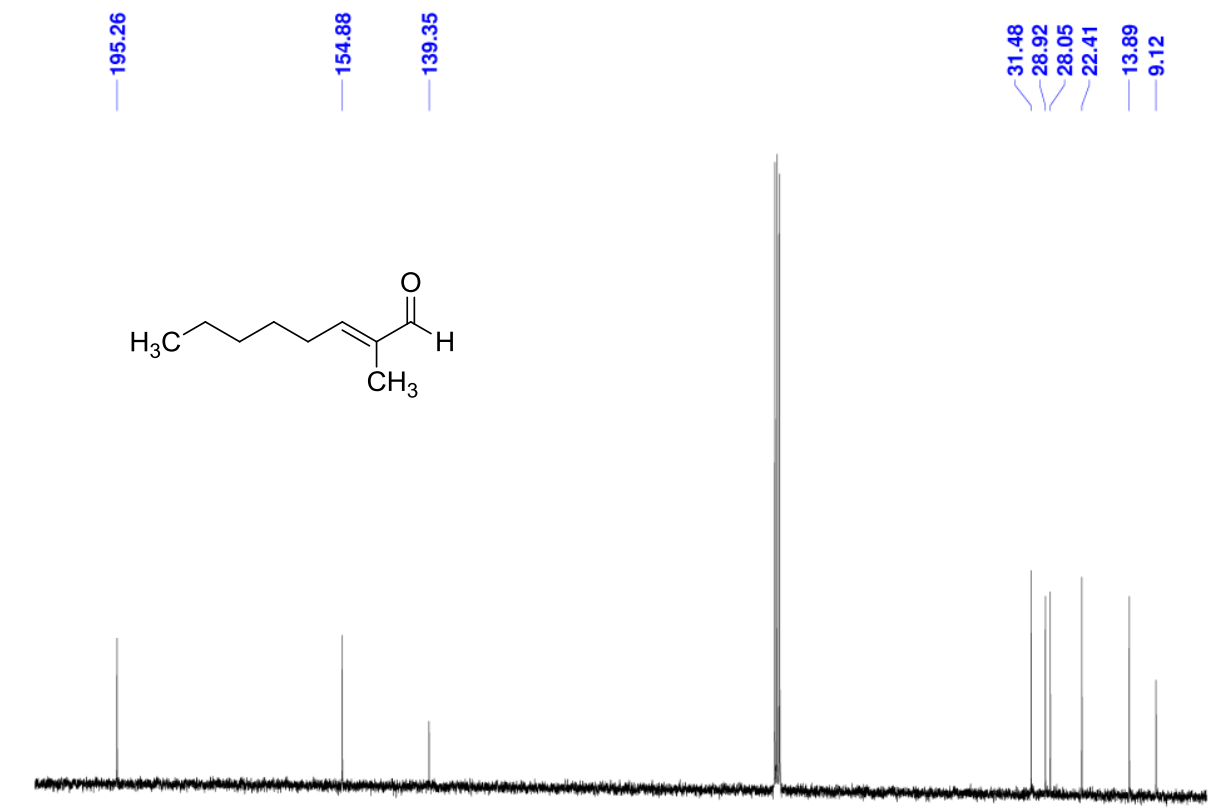

$\begin{array}{llllllllllllllllllll}200 & 190 & 180 & 170 & 160 & 150 & 140 & 130 & 120 & 110 & 100 & 90 & 80 & 70 & 60 & 50 & 40 & 30 & 20 & \mathrm{ppm}\end{array}$

Figure $S 77^{13} \mathrm{C}(150 \mathrm{MHz}) \mathrm{NMR}$ spectrum of $25 \mathrm{f}\left(\mathrm{CDCl}_{3}, 298 \mathrm{~K}\right)$. 


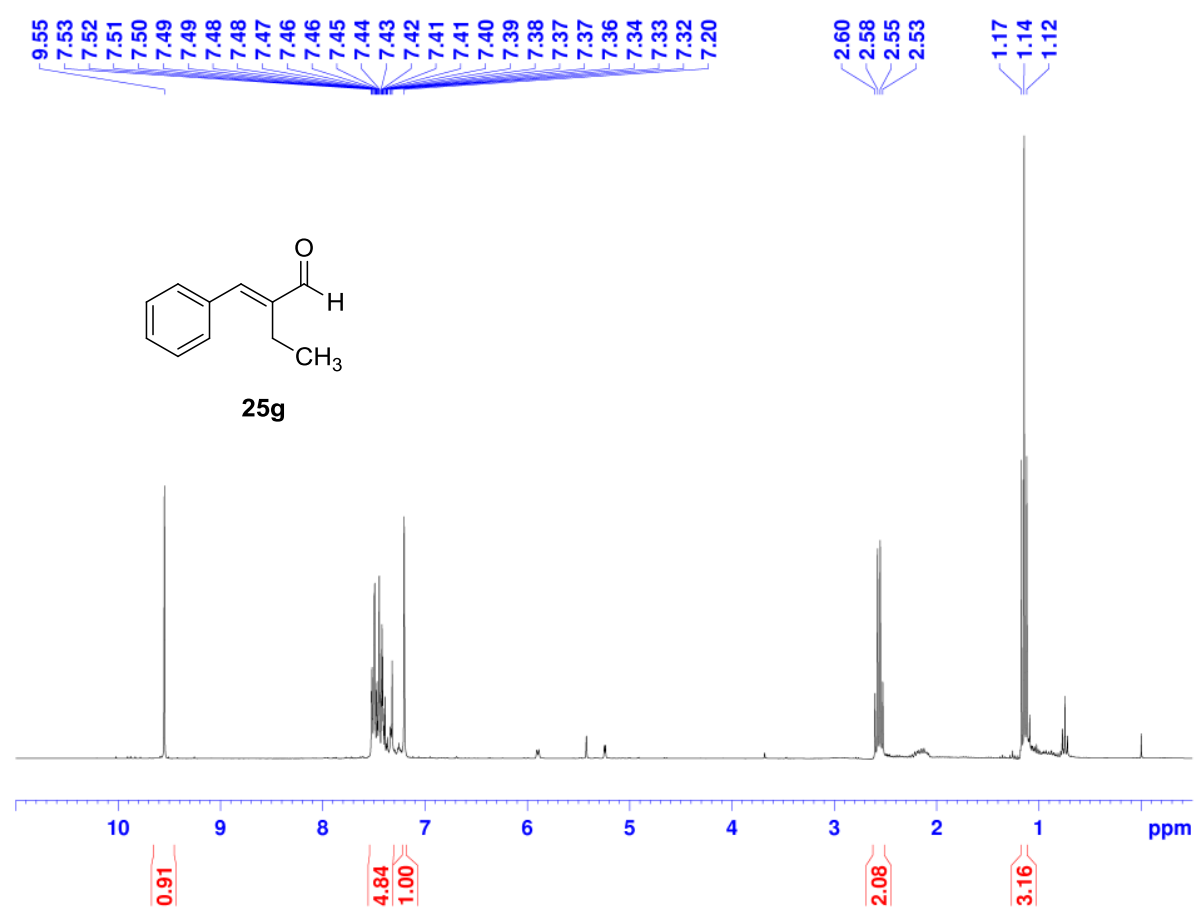

Figure $\mathbf{S 7 8}{ }^{1} \mathrm{H}(600 \mathrm{MHz}) \mathrm{NMR}$ spectrum of $\mathbf{2 5 g}\left(\mathrm{CDCl}_{3}, 298 \mathrm{~K}\right)$.

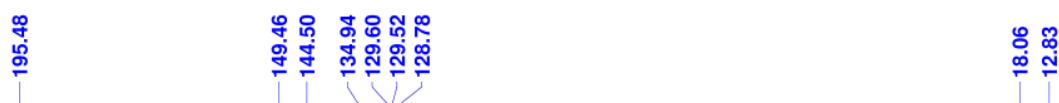<smiles>CC/C(C=O)=C\c1ccccc1</smiles>

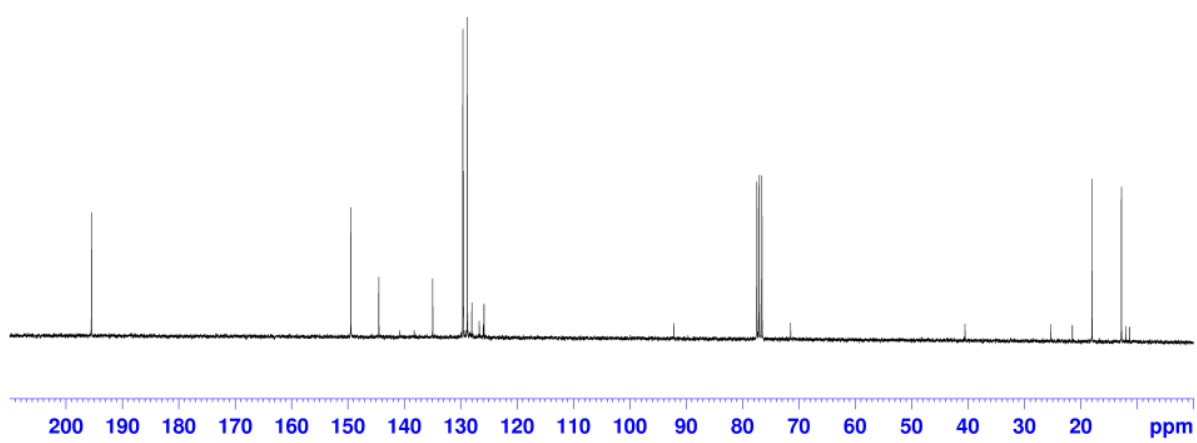

Figure $\mathbf{S 7 9}{ }^{13} \mathrm{C}(150 \mathrm{MHz}) \mathrm{NMR}$ spectrum of $\mathbf{2 5 g}\left(\mathrm{CDCl}_{3}, 298 \mathrm{~K}\right)$. 


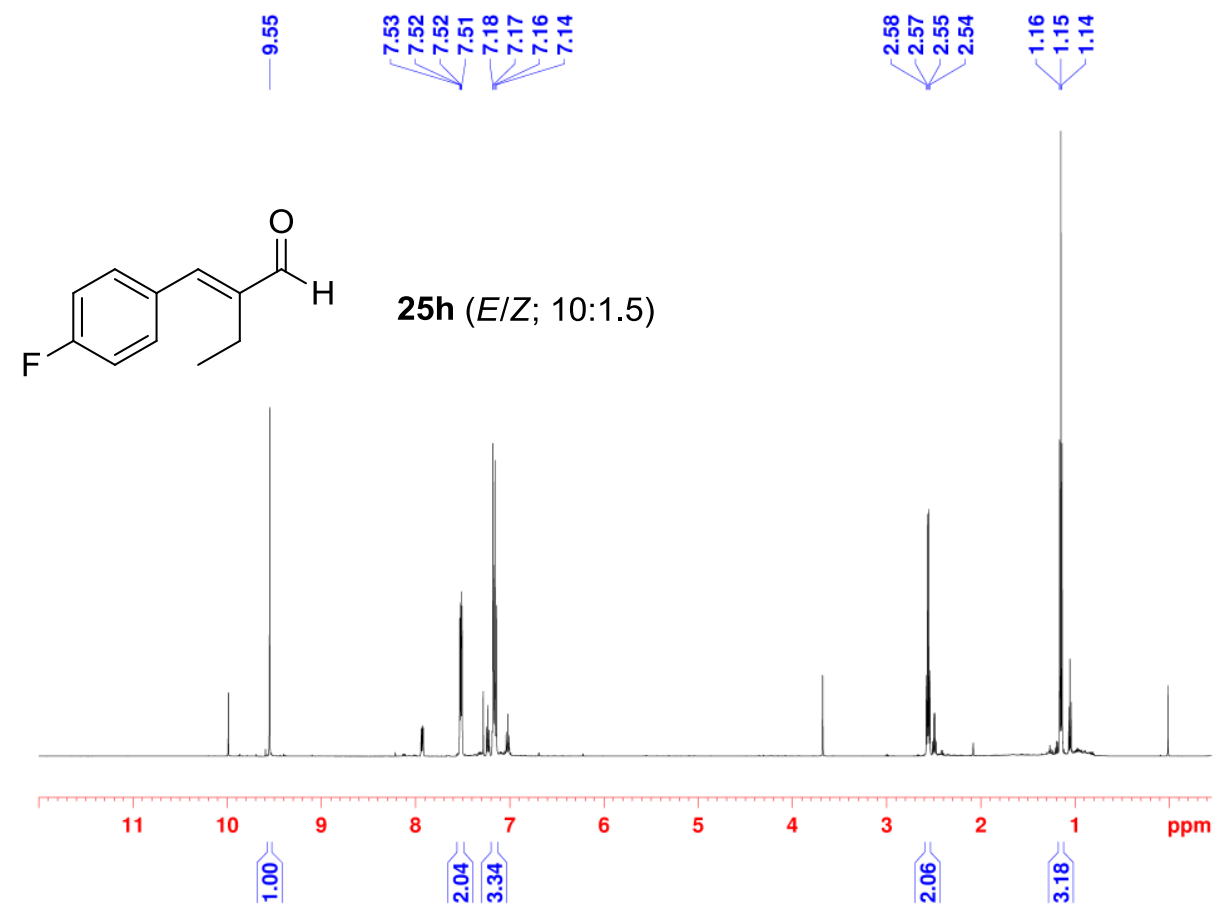

Figure $580^{1} \mathrm{H}(600 \mathrm{MHz}) \mathrm{NMR}$ spectrum of $25 \mathrm{~h}\left(\mathrm{CDCl}_{3}, 298 \mathrm{~K}\right)$.

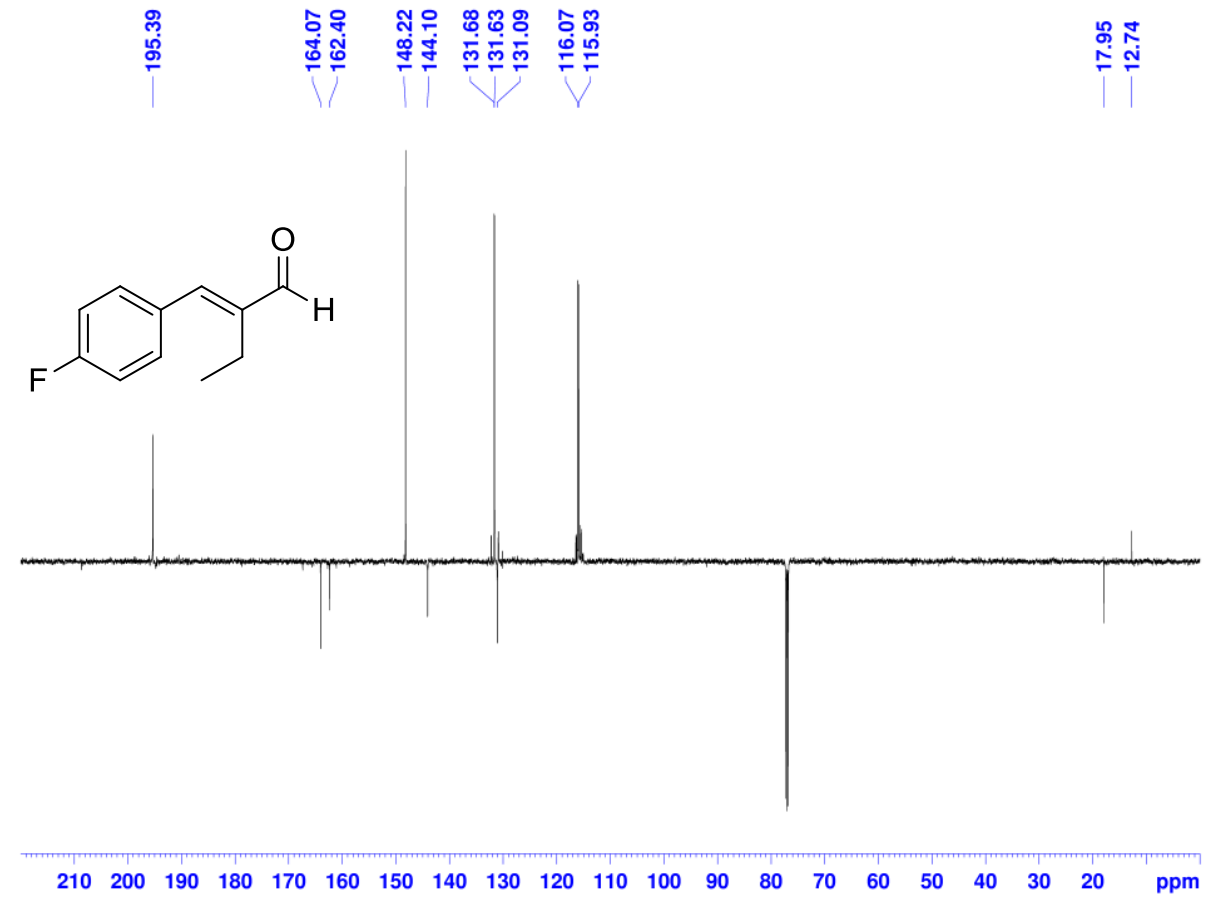

Figure $S 81{ }^{13} \mathrm{C}(150 \mathrm{MHz})$ DEPTQ NMR spectrum of $25 \mathrm{~h}\left(\mathrm{CDCl}_{3}, 298 \mathrm{~K}\right)$. 


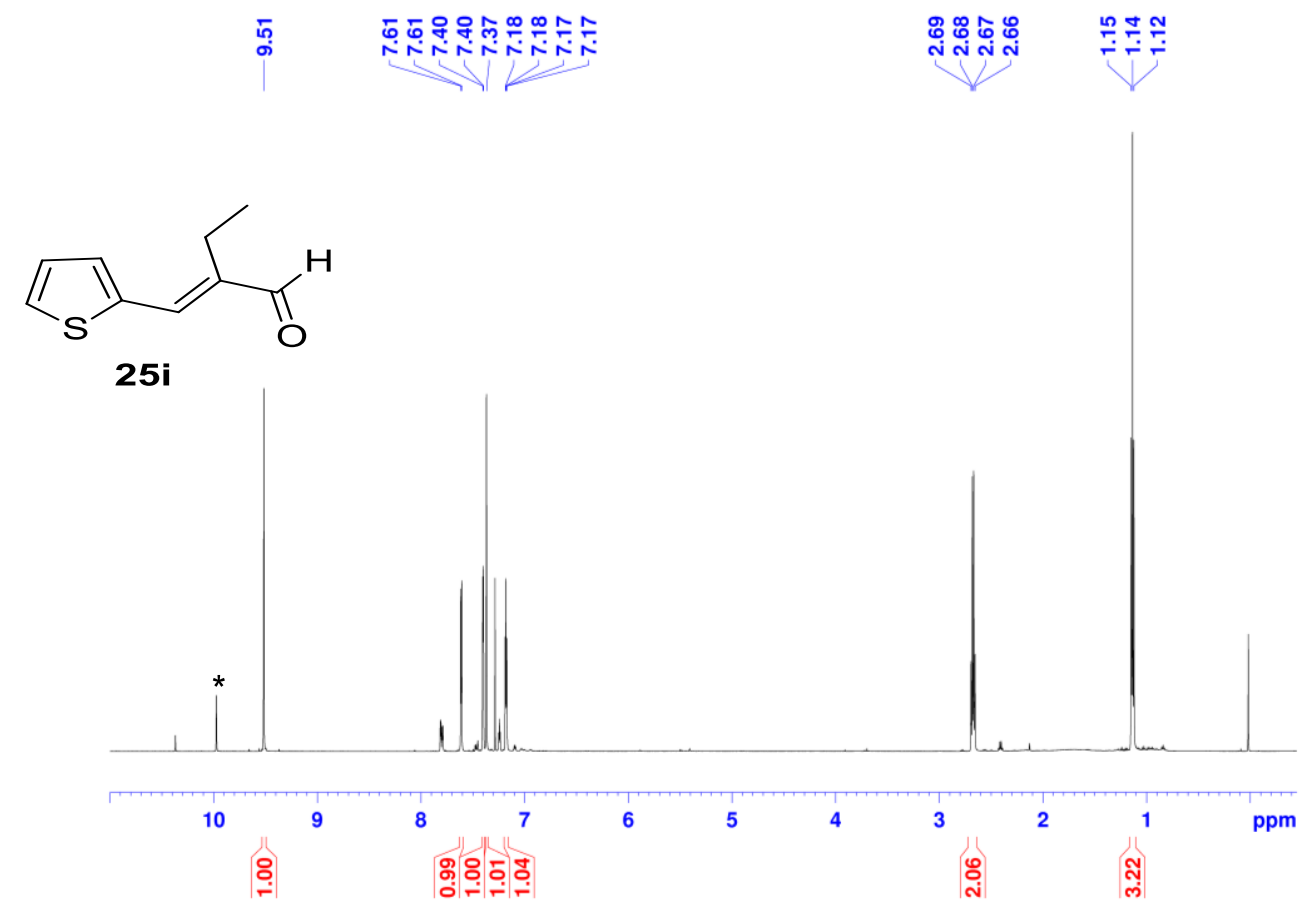

Figure $S 82{ }^{1} \mathrm{H}(600 \mathrm{MHz}) \mathrm{NMR}$ spectrum of $25 \mathrm{i}$ ( ${ }^{*}$ containing unidentified impurity $)\left(\mathrm{CDCl}_{3}\right.$, $298 \mathrm{~K})$.

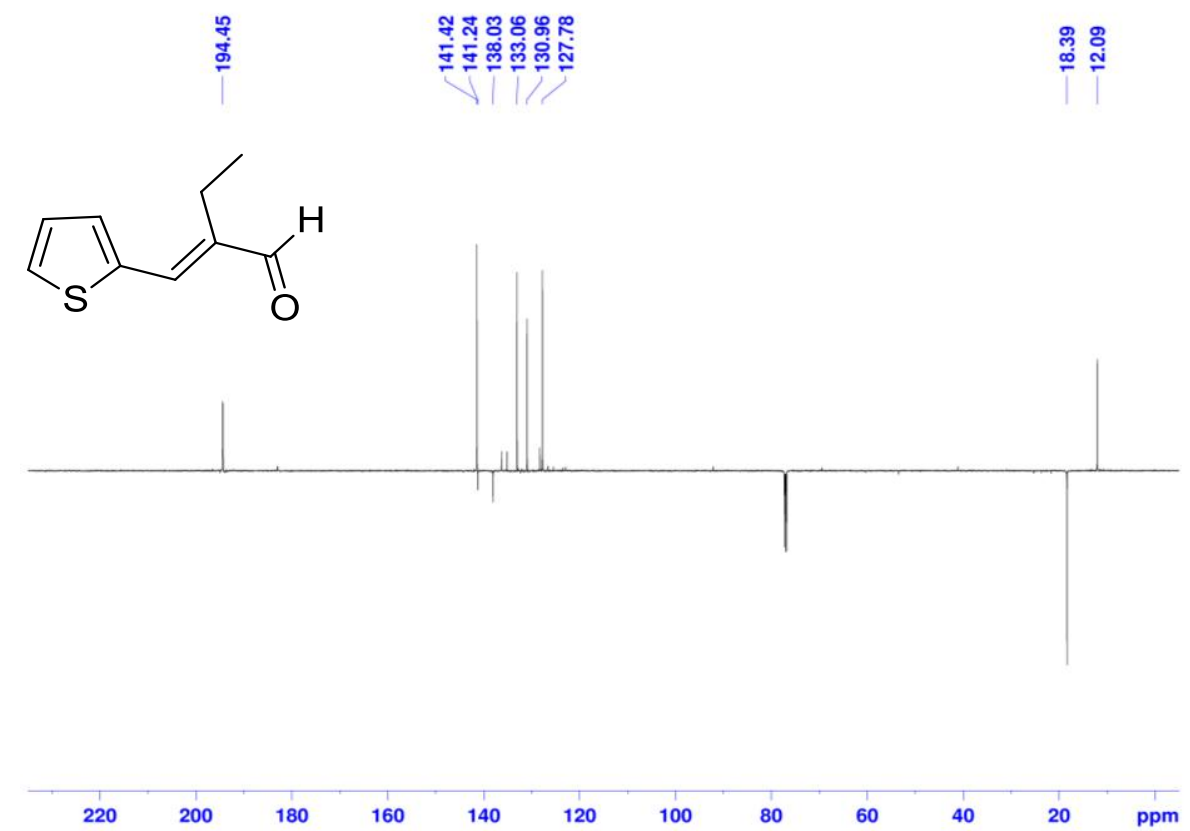

Figure $583{ }^{13} \mathrm{C}(150 \mathrm{MHz})$ DEPTQ NMR spectrum of $25 \mathrm{i}\left({ }^{*}\right.$ containing unidentified impurity $\left(\mathrm{CDCl}_{3}, 298 \mathrm{~K}\right)$. 


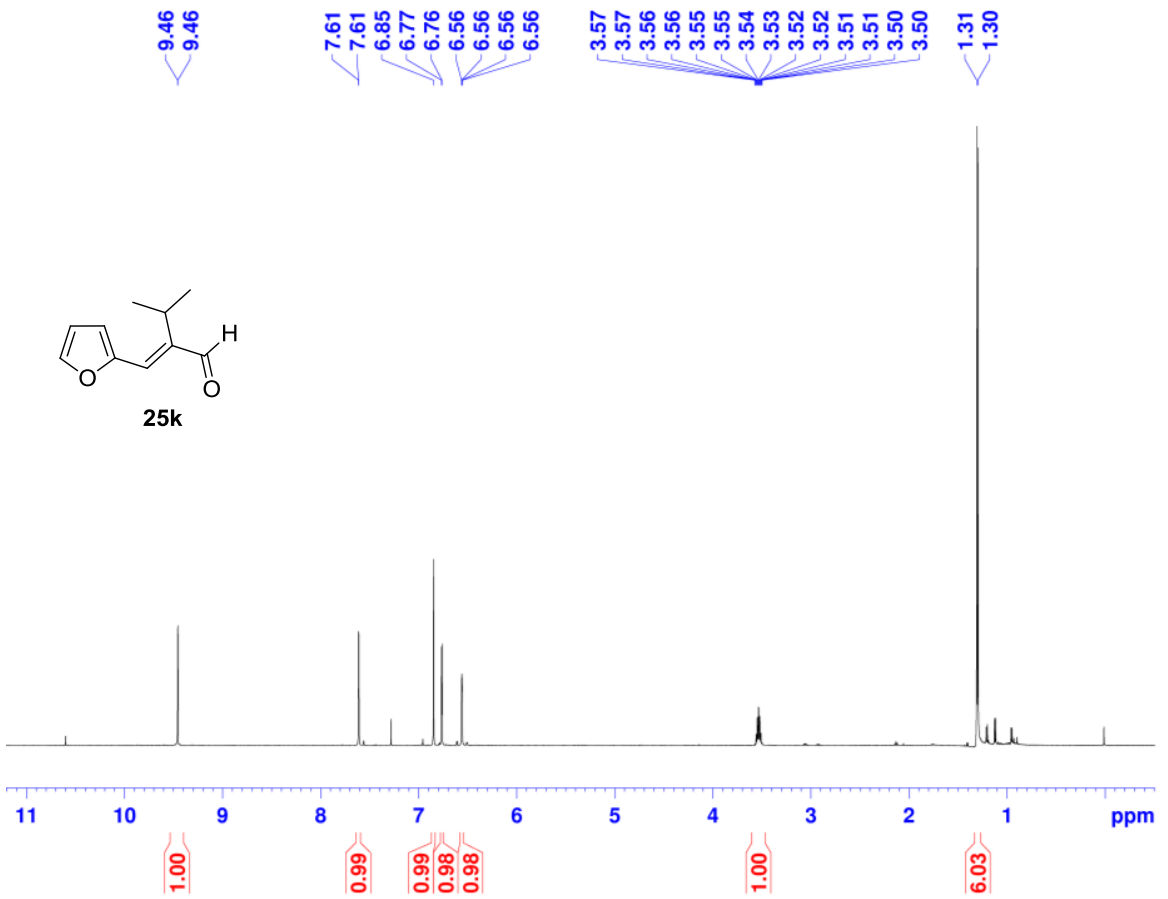

Figure $\mathbf{S 8 4}{ }^{1} \mathrm{H}(600 \mathrm{MHz}) \mathrm{NMR}$ spectrum of $25 \mathrm{k}\left(\mathrm{CDCl}_{3}, 298 \mathrm{~K}\right)$.

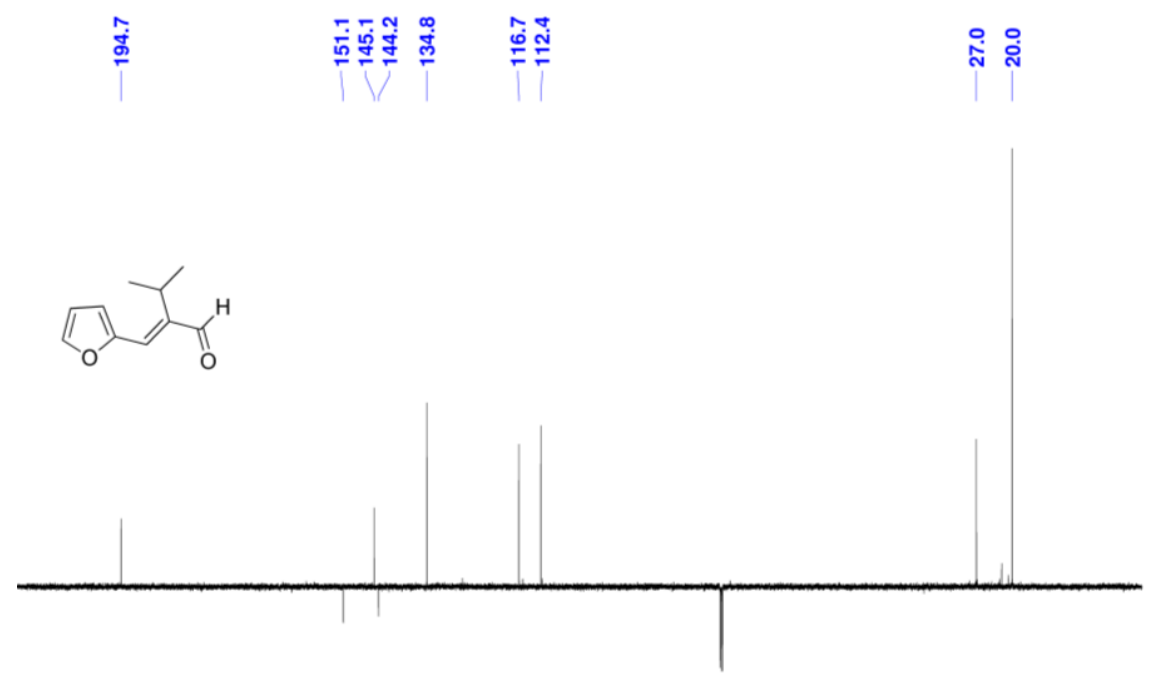

$\begin{array}{llllllllllllllllllllll}210 & 200 & 190 & 180 & 170 & 160 & 150 & 140 & 130 & 120 & 110 & 100 & 90 & 80 & 70 & 60 & 50 & 40 & 30 & 20 & 10 & \mathrm{ppm}\end{array}$

Figure $\mathbf{S 8 5}{ }^{13} \mathrm{C}(150 \mathrm{MHz})$ DEPTQ NMR spectrum of 25k $\left(\mathrm{CDCl}_{3}, 298 \mathrm{~K}\right)$. 


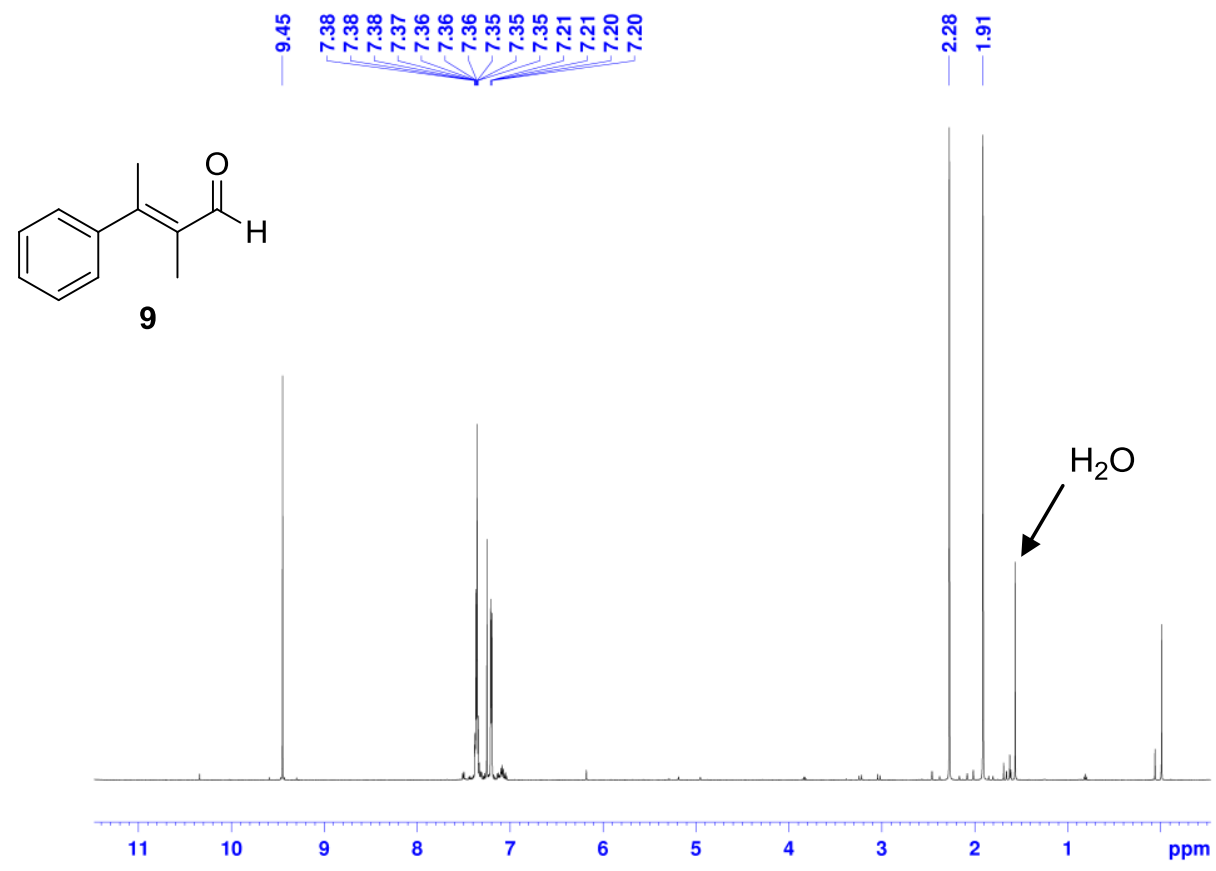

Figure $\mathrm{S} 86{ }^{1} \mathrm{H}(600 \mathrm{MHz}) \mathrm{NMR}$ spectrum of $9\left(\mathrm{CDCl}_{3}, 298 \mathrm{~K}\right)$.

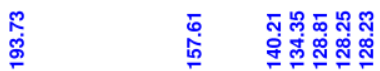

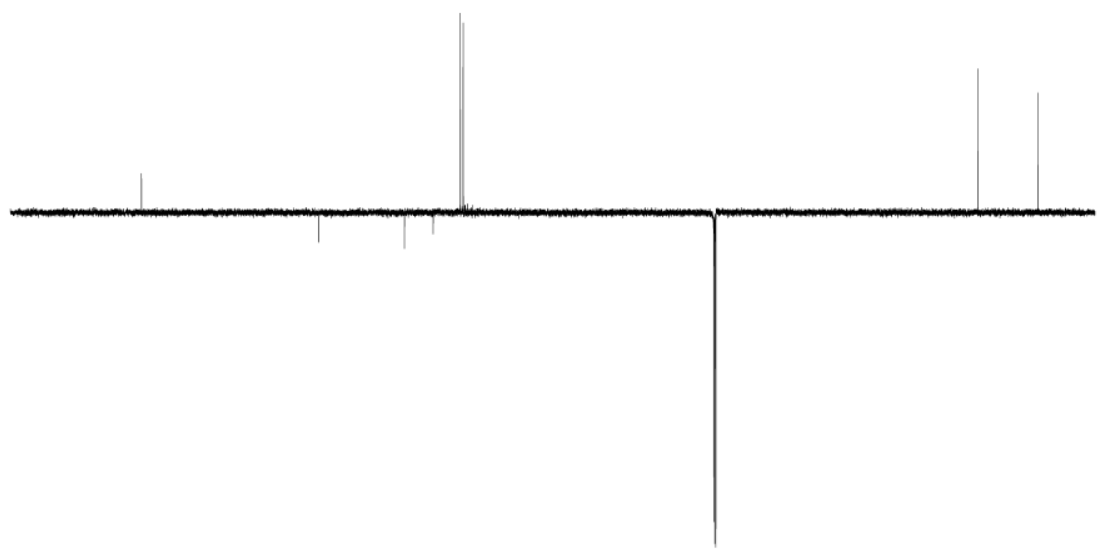

$\begin{array}{lllllllllllllllllllll}210 & 200 & 190 & 180 & 170 & 160 & 150 & 140 & 130 & 120 & 110 & 100 & 90 & 80 & 70 & 60 & 50 & 40 & 30 & 20 & \mathrm{ppm}\end{array}$

Figure $\mathbf{S} 87^{1} \mathrm{H}(150 \mathrm{MHz})$ DEPTQ NMR spectrum of $9\left(\mathrm{CDCl}_{3}, 298 \mathrm{~K}\right)$. 


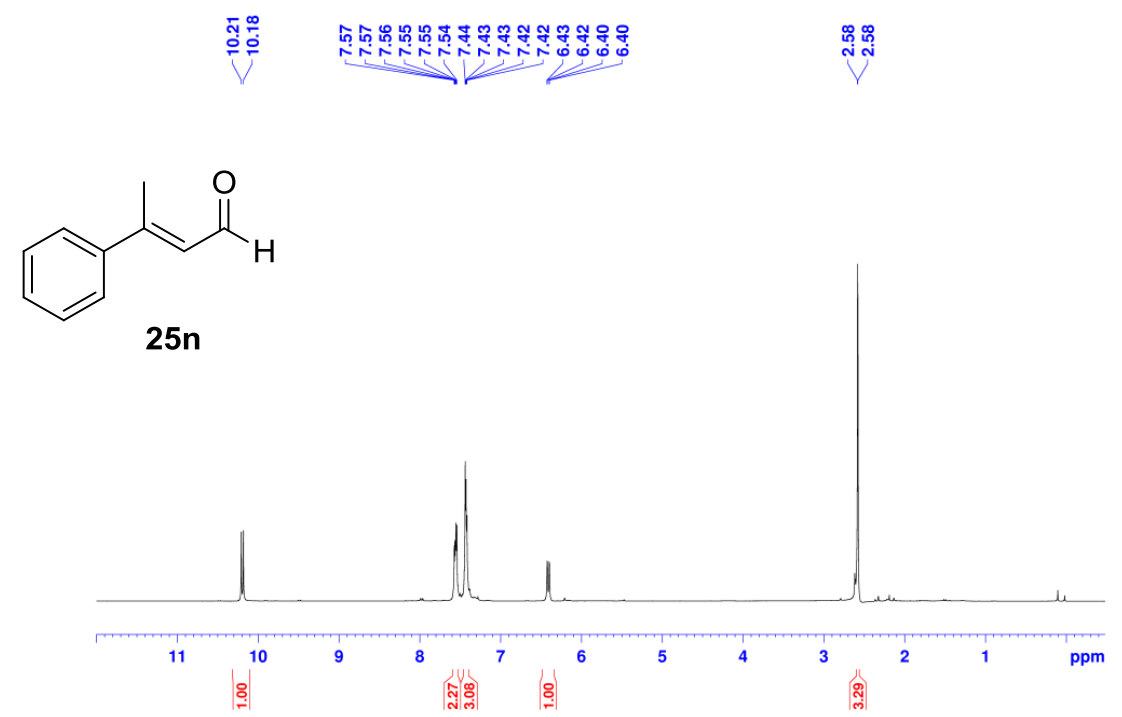

Figure $\mathbf{S} 88^{1} \mathrm{H}(300 \mathrm{MHz}) \mathrm{NMR}$ spectrum of $25 \mathrm{n}\left(\mathrm{CDCl}_{3}, 298 \mathrm{~K}\right)$.

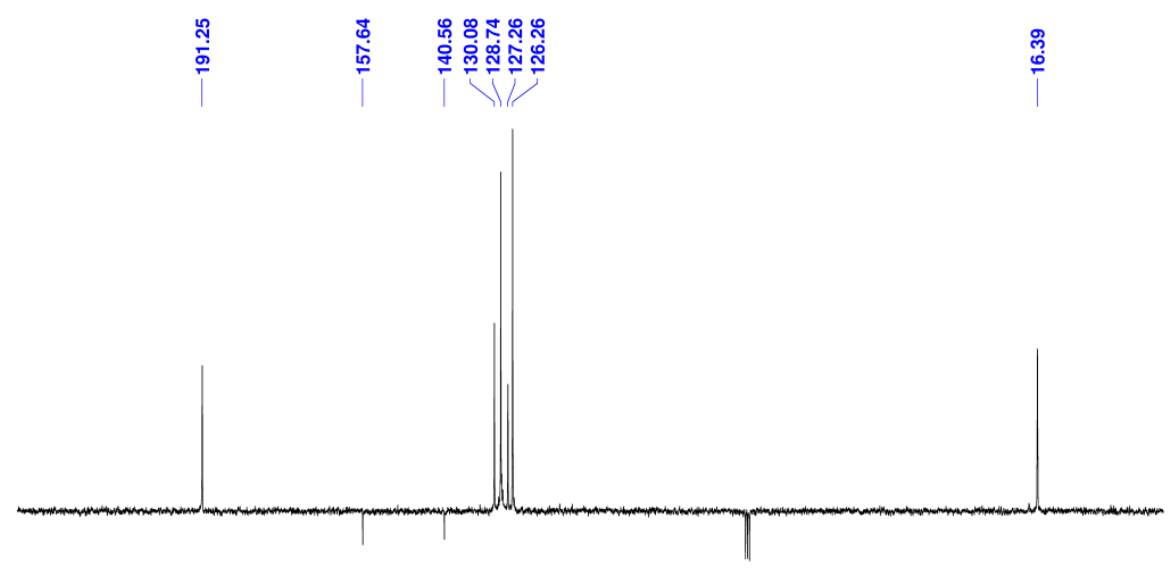

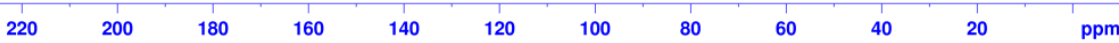

Figure $589{ }^{1} \mathrm{H}(75 \mathrm{MHz})$ DEPTQ NMR spectrum of $25 \mathbf{n}\left(\mathrm{CDCl}_{3}, 298 \mathrm{~K}\right)$. 


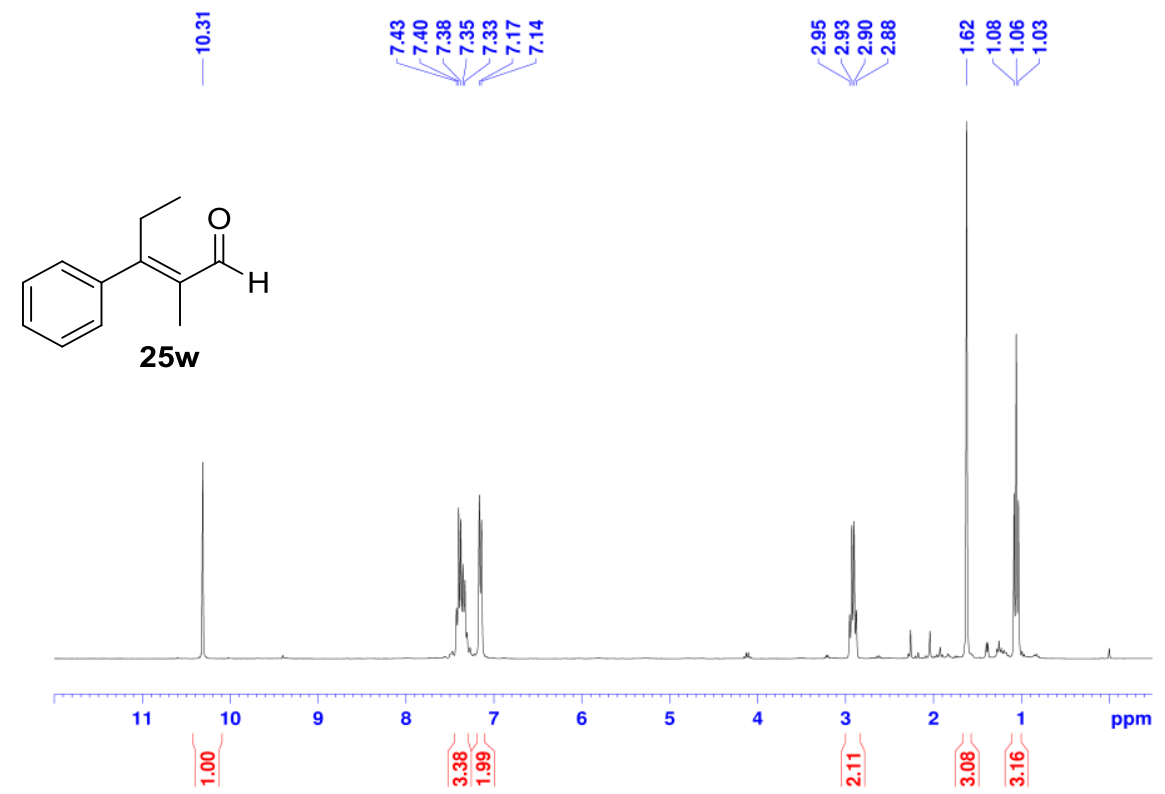

Figure $590^{1} \mathrm{H}(600 \mathrm{MHz}) \mathrm{NMR}$ spectrum of $25 \mathrm{w}\left(\mathrm{CDCl}_{3}, 298 \mathrm{~K}\right)$.
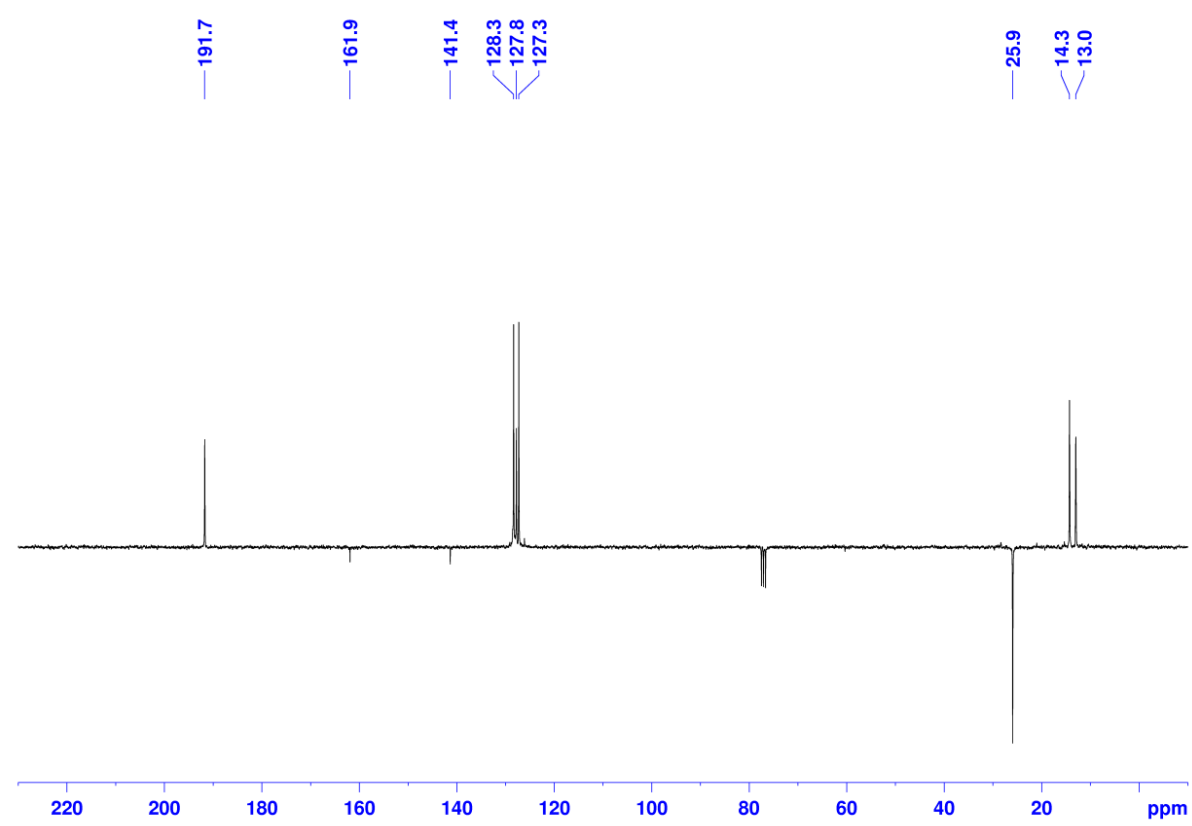

Figure $591{ }^{13} \mathrm{C}(150 \mathrm{MHz})$ DEPTQ NMR spectrum of $25 \mathbf{w}\left(\mathrm{CDCl}_{3}, 298 \mathrm{~K}\right)$. 


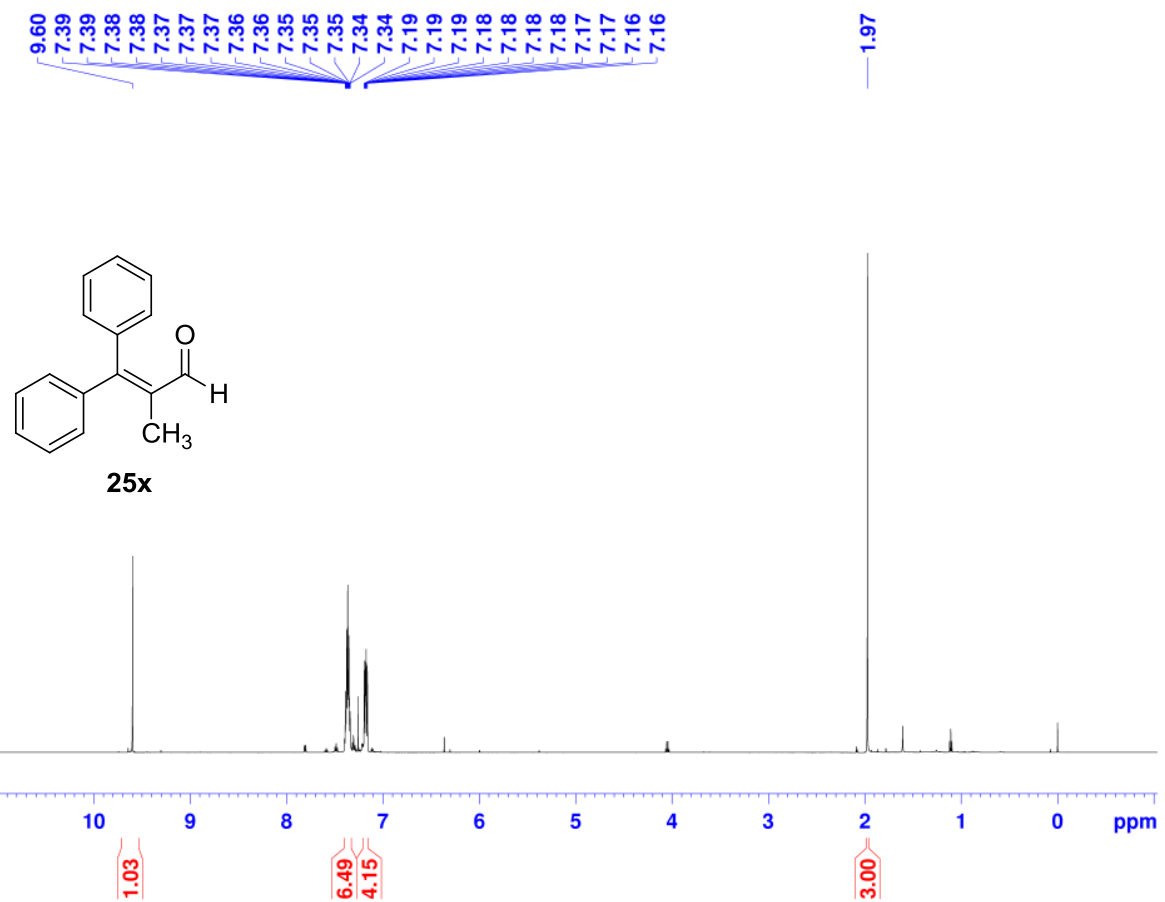

Figure $S 92{ }^{1} \mathrm{H}(150 \mathrm{MHz}) \mathrm{NMR}$ spectrum of $25 \times\left(\mathrm{CDCl}_{3}, 298 \mathrm{~K}\right)$.

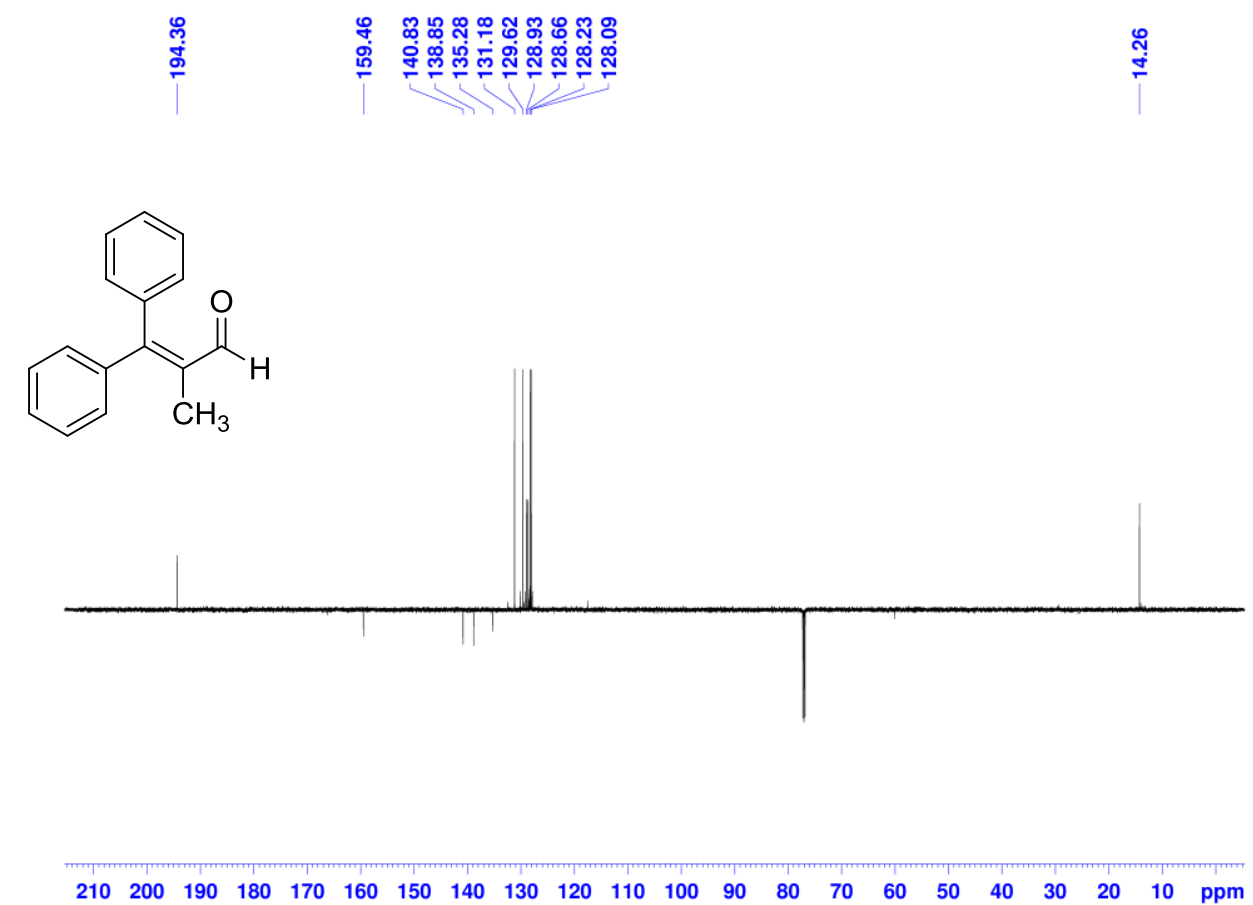

Figure $\mathbf{S 9 3}{ }^{13} \mathrm{C}(150 \mathrm{MHz})$ DEPTQ NMR spectrum of 25x $\left(\mathrm{CDCl}_{3}, 298 \mathrm{~K}\right)$. 


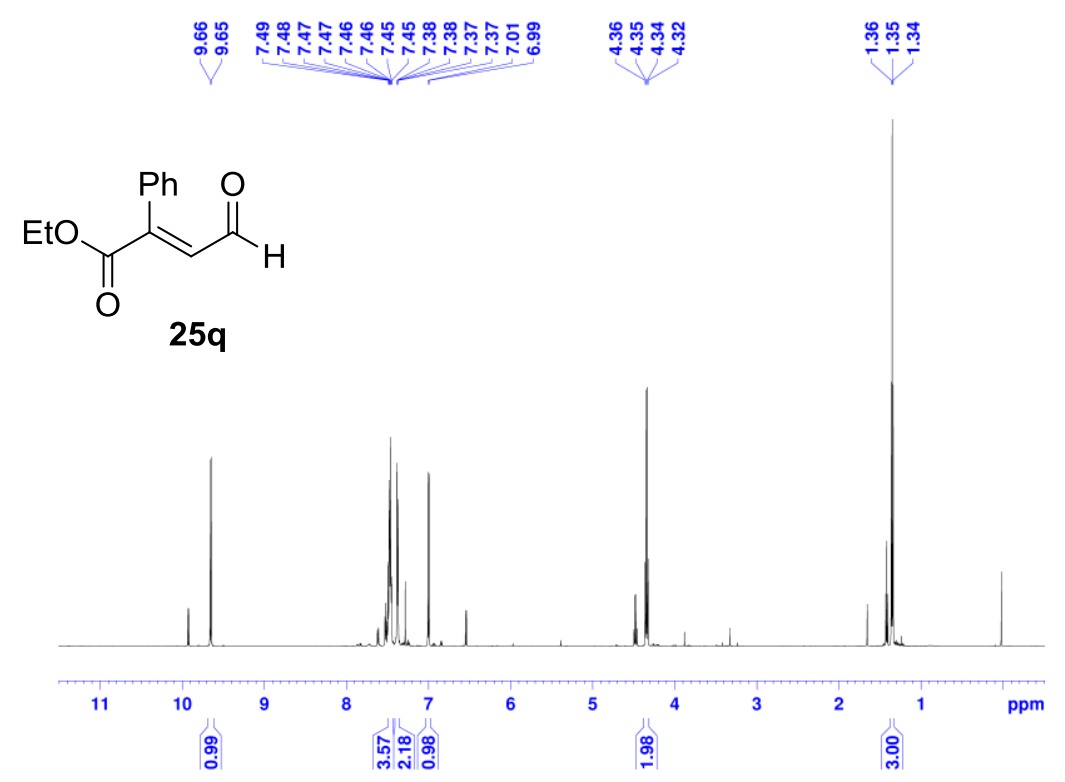

Figure $594{ }^{1} \mathrm{H}(150 \mathrm{MHz}) \mathrm{NMR}$ spectrum of $\mathbf{2 5 q}\left(\mathrm{CDCl}_{3}, 298 \mathrm{~K}\right)$.

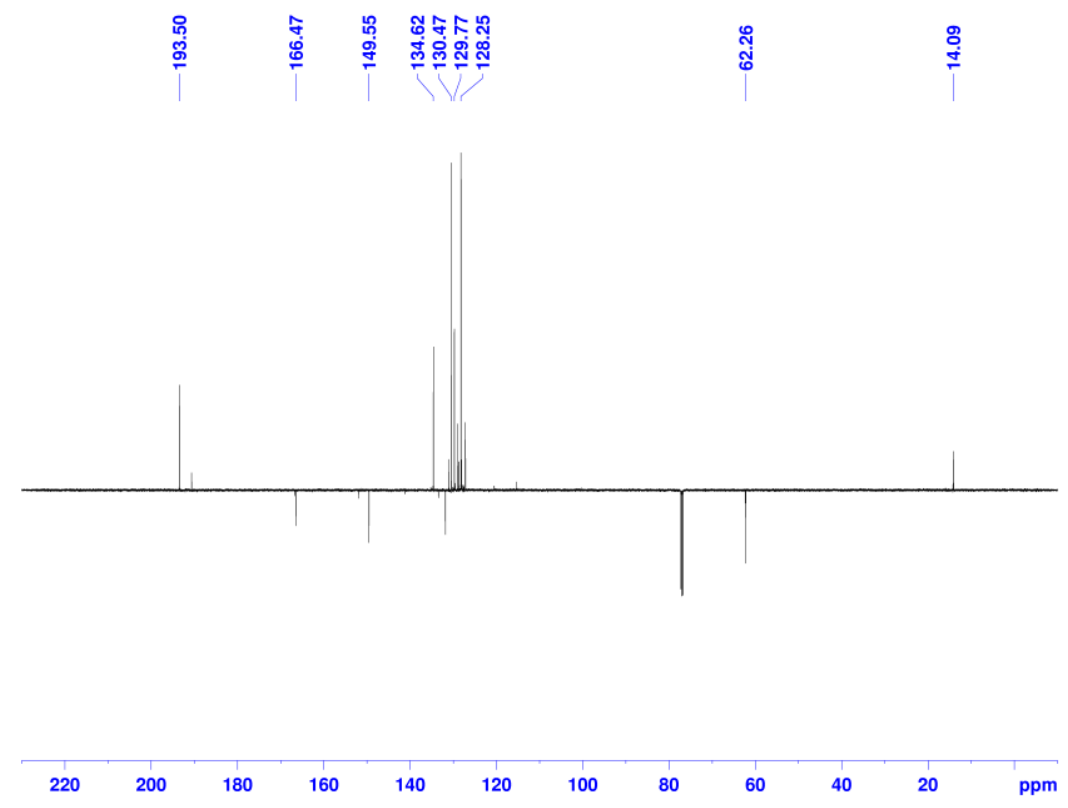

Figure $595{ }^{13} \mathrm{C}(150 \mathrm{MHz})$ DEPTQ NMR spectrum of $25 \mathrm{q}\left(\mathrm{CDCl}_{3}, 298 \mathrm{~K}\right)$. 


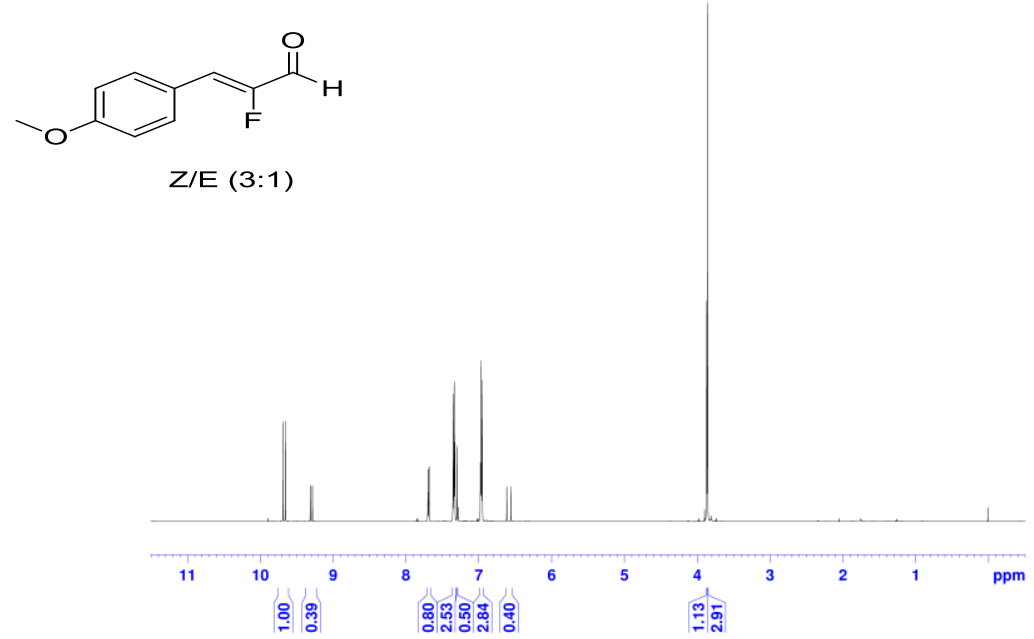

Figure $596{ }^{1} \mathrm{H}(150 \mathrm{MHz}) \mathrm{NMR}$ spectrum of $25 \mathrm{~m}\left(\mathrm{CDCl}_{3}, 298 \mathrm{~K}\right)$.

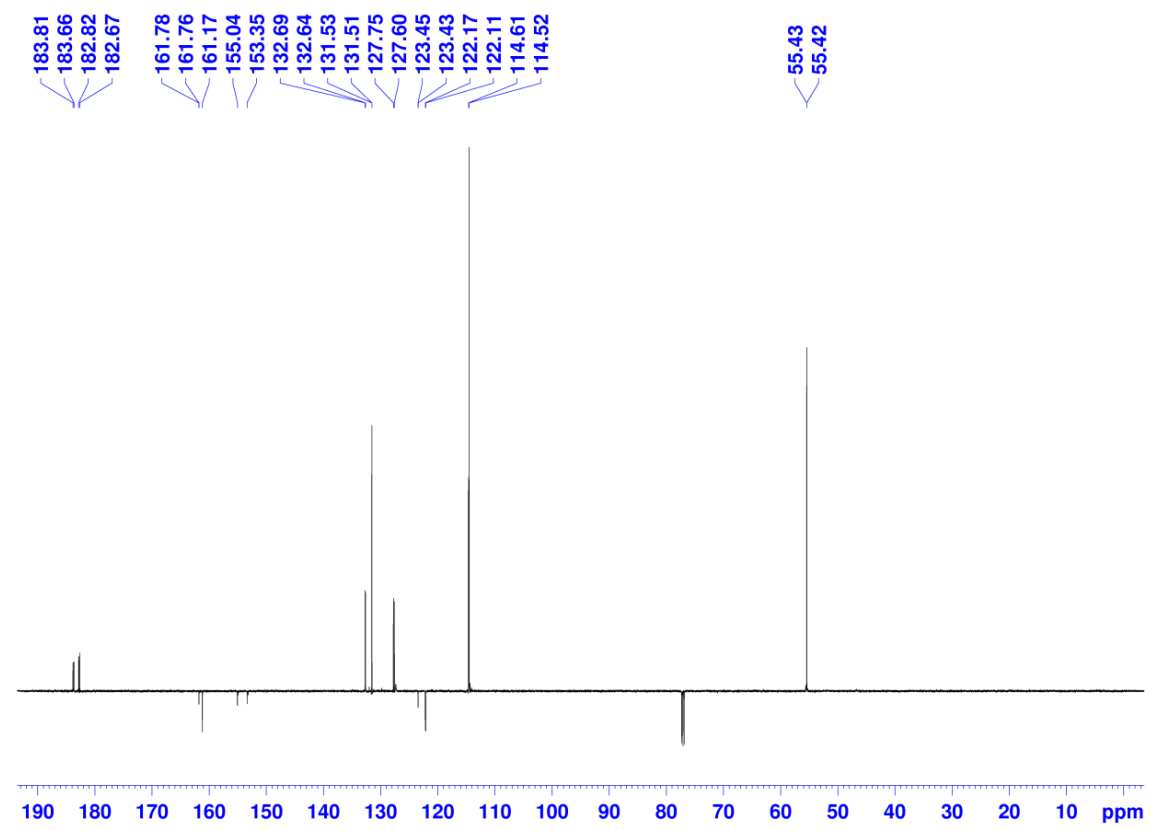

Figure $\mathbf{S 9 7} \mathbf{1}^{13} \mathrm{C}(150 \mathrm{MHz})$ DEPTQ NMR spectrum of $25 \mathrm{~m}\left(\mathrm{CDCl}_{3}, 298 \mathrm{~K}\right)$. 


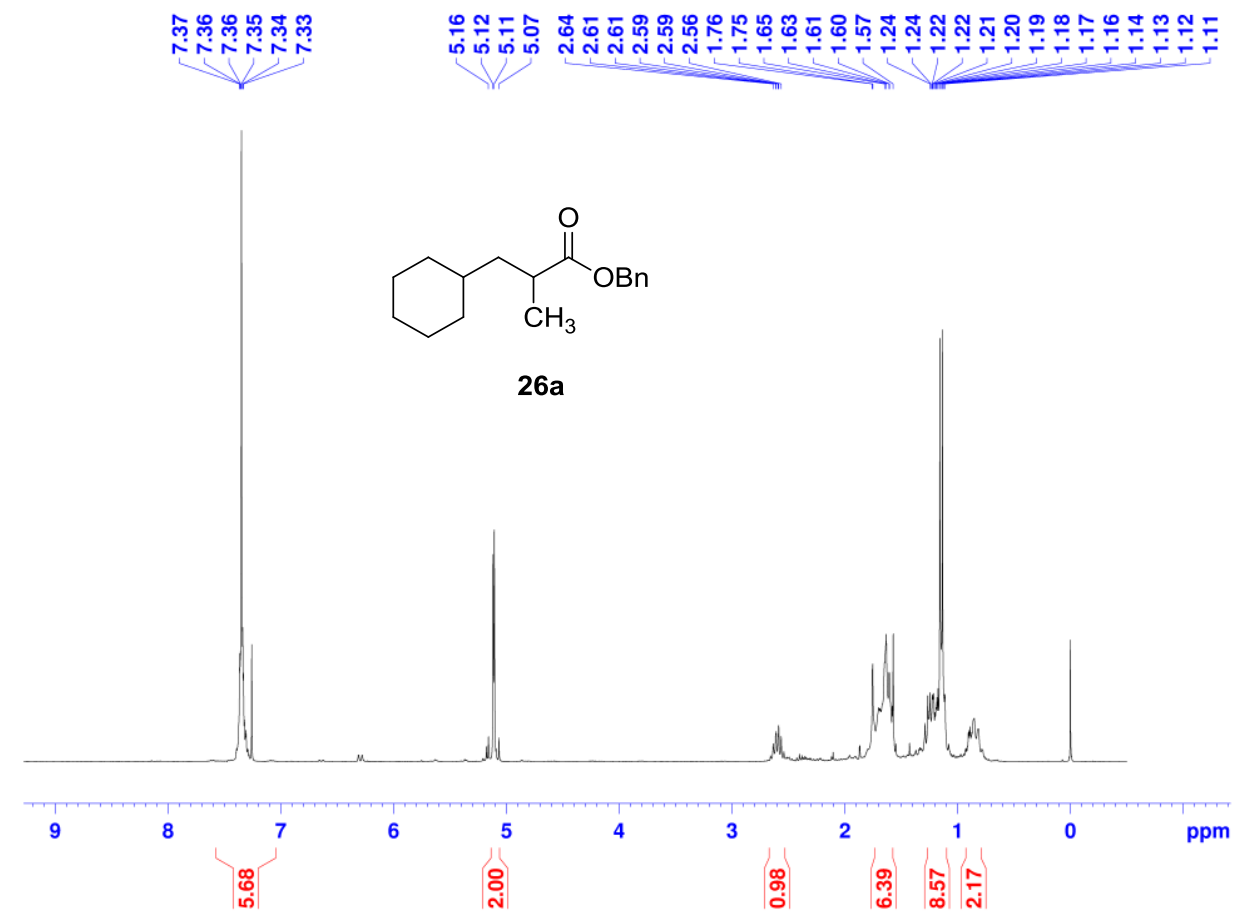

Figure $598{ }^{1} \mathrm{H}(600 \mathrm{MHz}) \mathrm{NMR}$ spectrum of $\mathbf{2 6 a}\left(\mathrm{CDCl}_{3}, 298 \mathrm{~K}\right)$.

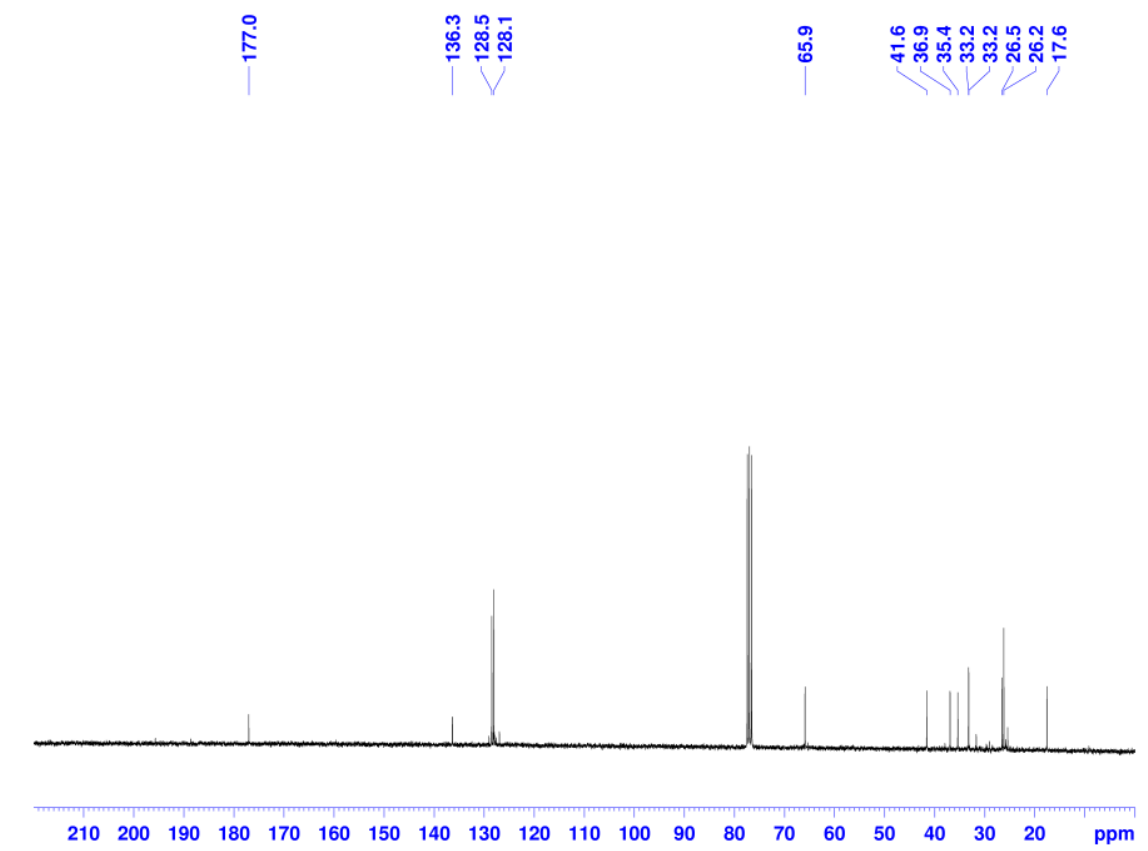

Figure $\mathbf{S 9 9}{ }^{13} \mathrm{C}(150 \mathrm{MHz}) \mathrm{NMR}$ spectrum of 26a $\left(\mathrm{CDCl}_{3}, 298 \mathrm{~K}\right)$. 
<smiles>CC(Cc1ccccc1)C(=O)OCc1ccccc1</smiles>

26b

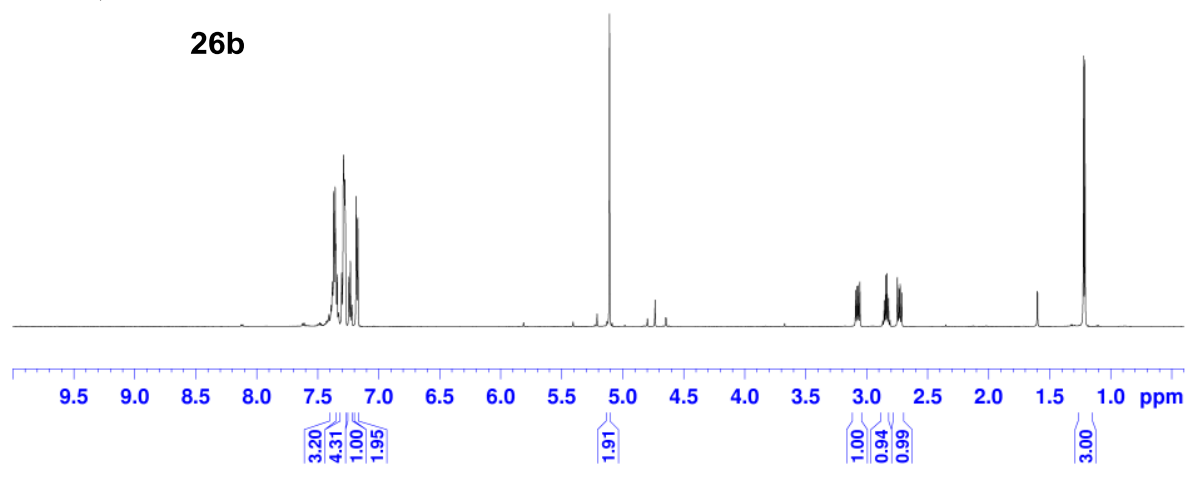

Figure $5100{ }^{1} \mathrm{H}(600 \mathrm{MHz}) \mathrm{NMR}$ spectrum of $\mathbf{2 6 b}\left(\mathrm{CDCl}_{3}, 298 \mathrm{~K}\right)$.

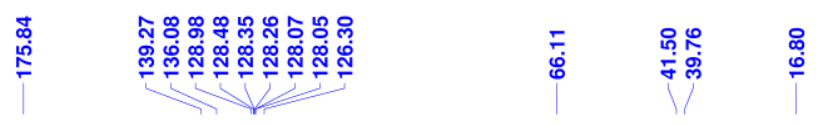<smiles>CC(Cc1ccccc1)C(=O)OCc1ccccc1</smiles>

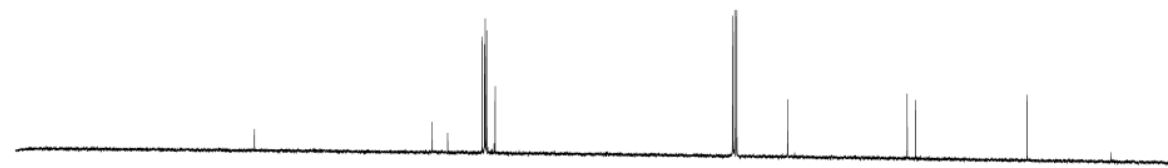

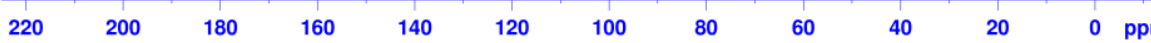

Figure $\mathrm{S} 101{ }^{13} \mathrm{C}(150 \mathrm{MHz}) \mathrm{NMR}$ spectrum of $26 \mathrm{~b}\left(\mathrm{CDCl}_{3}, 298 \mathrm{~K}\right)$. 


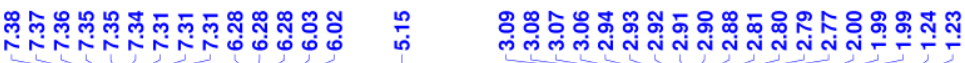<smiles>CCCCOC(=O)C(C)Cc1ccco1</smiles>

26c

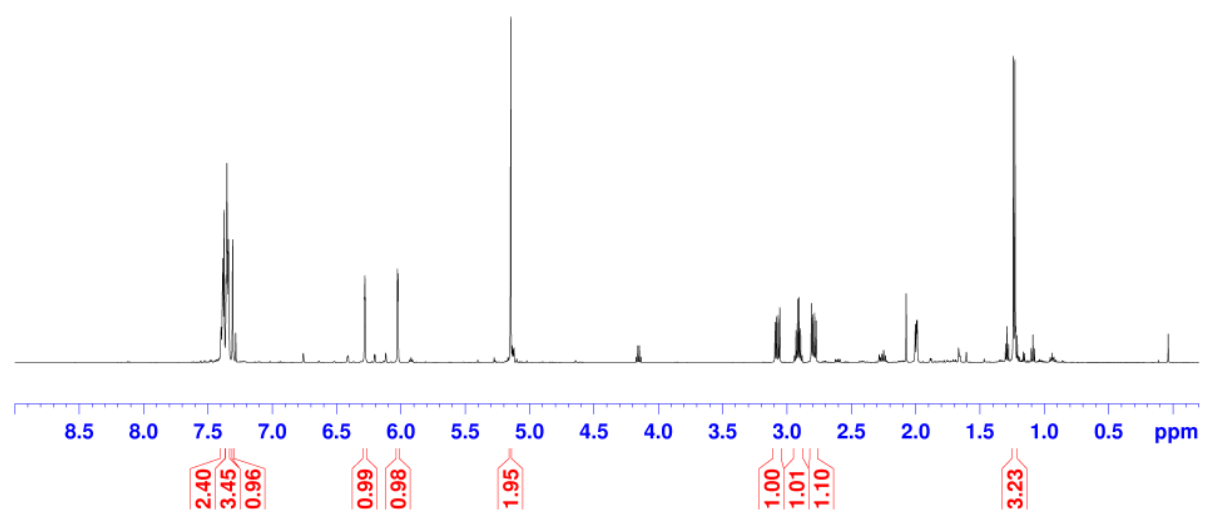

Figure $5102{ }^{13} \mathrm{C}(600 \mathrm{MHz}) \mathrm{NMR}$ spectrum of $26 \mathrm{c}\left(\mathrm{CDCl}_{3}, 298 \mathrm{~K}\right)$.

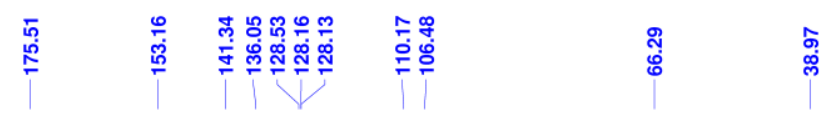

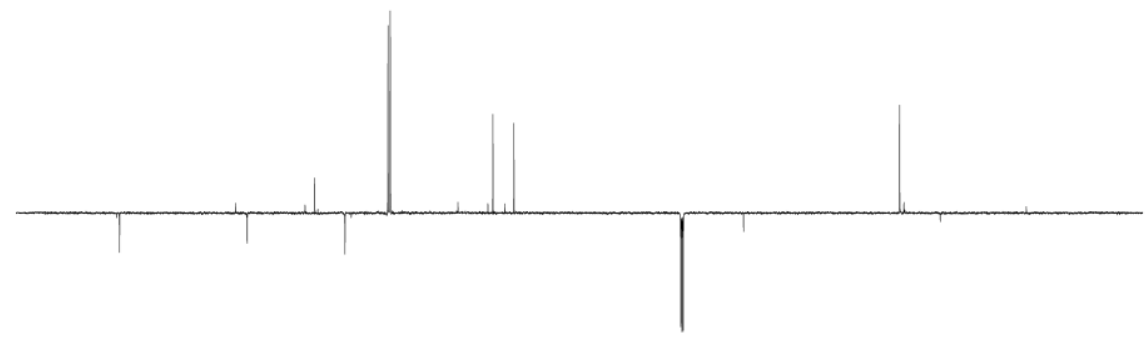

$\begin{array}{llllllllllllllllllll}190 & 180 & 170 & 160 & 150 & 140 & 130 & 120 & 110 & 100 & 90 & 80 & 70 & 60 & 50 & 40 & 30 & 20 & 10 & \mathrm{ppm}\end{array}$

Figure $\mathbf{S 1 0 3}{ }^{13} \mathrm{C}(150 \mathrm{MHz})$ DEPTQ NMR spectrum of $\mathbf{2 6 c}\left(\mathrm{CDCl}_{3}, 298 \mathrm{~K}\right)$. 


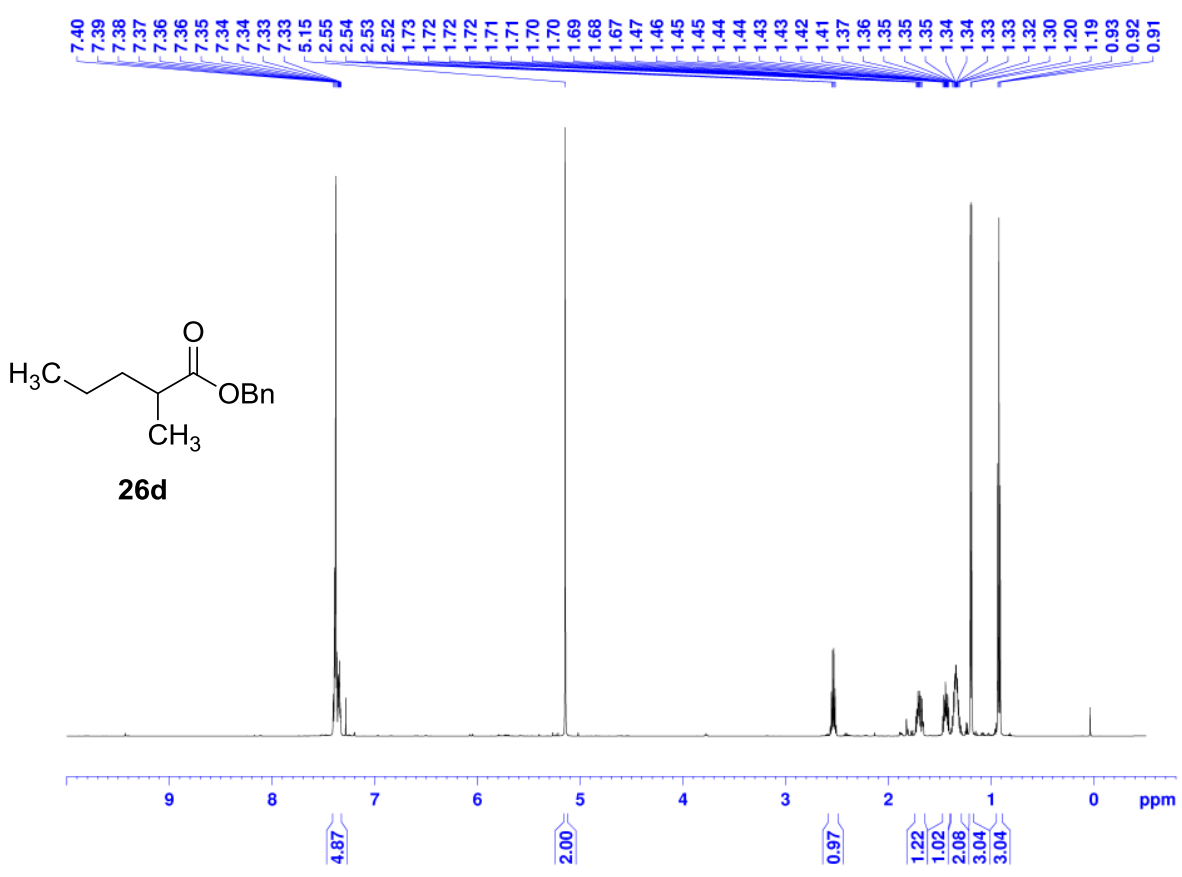

Figure $5104{ }^{1} \mathrm{H}(600 \mathrm{MHz}) \mathrm{NMR}$ spectrum of $\mathbf{2 6 d}\left(\mathrm{CDCl}_{3}, 298 \mathrm{~K}\right)$.

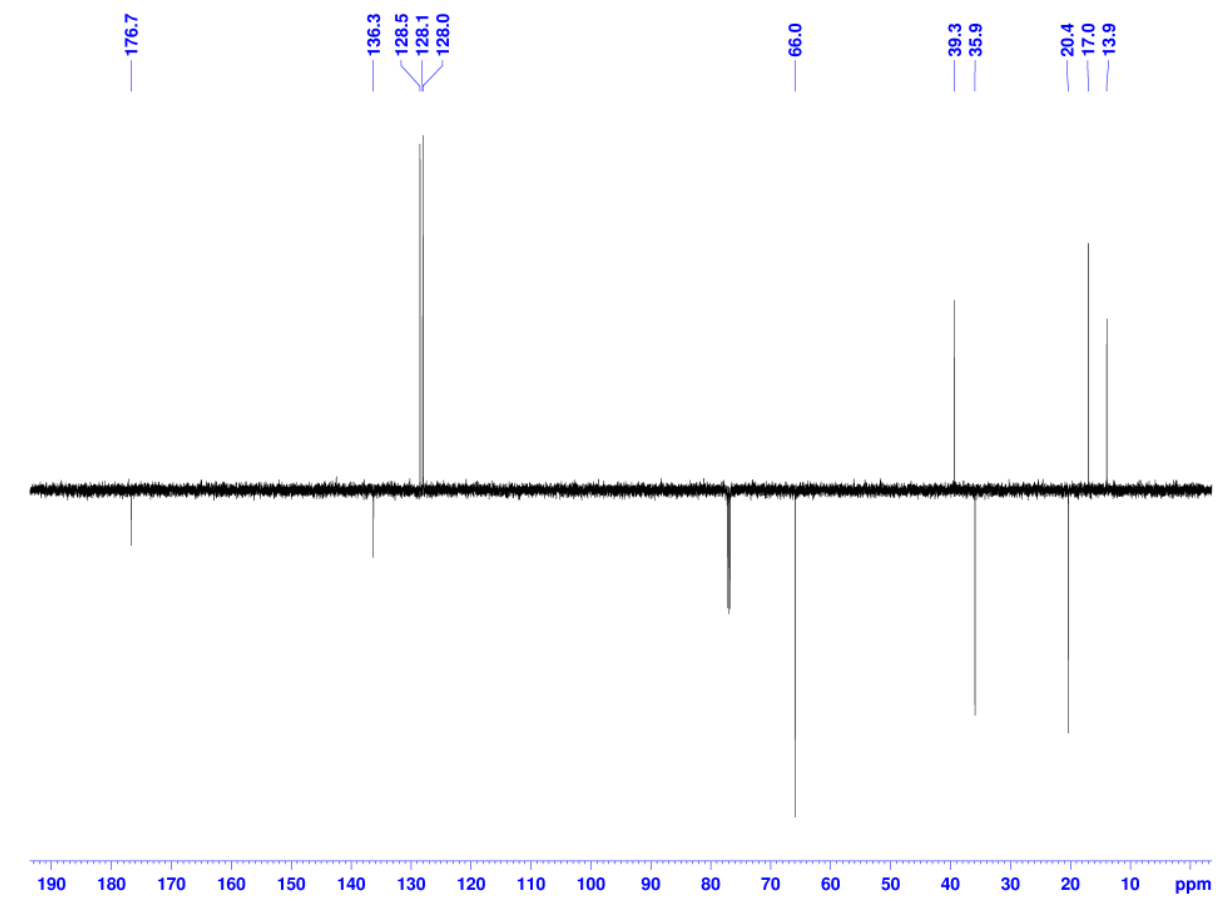

Figure $\mathbf{S 1 0 5}{ }^{13} \mathrm{C}(150 \mathrm{MHz})$ DEPTQ NMR spectrum of $\mathbf{2 6 d}\left(\mathrm{CDCl}_{3}, 298 \mathrm{~K}\right)$. 


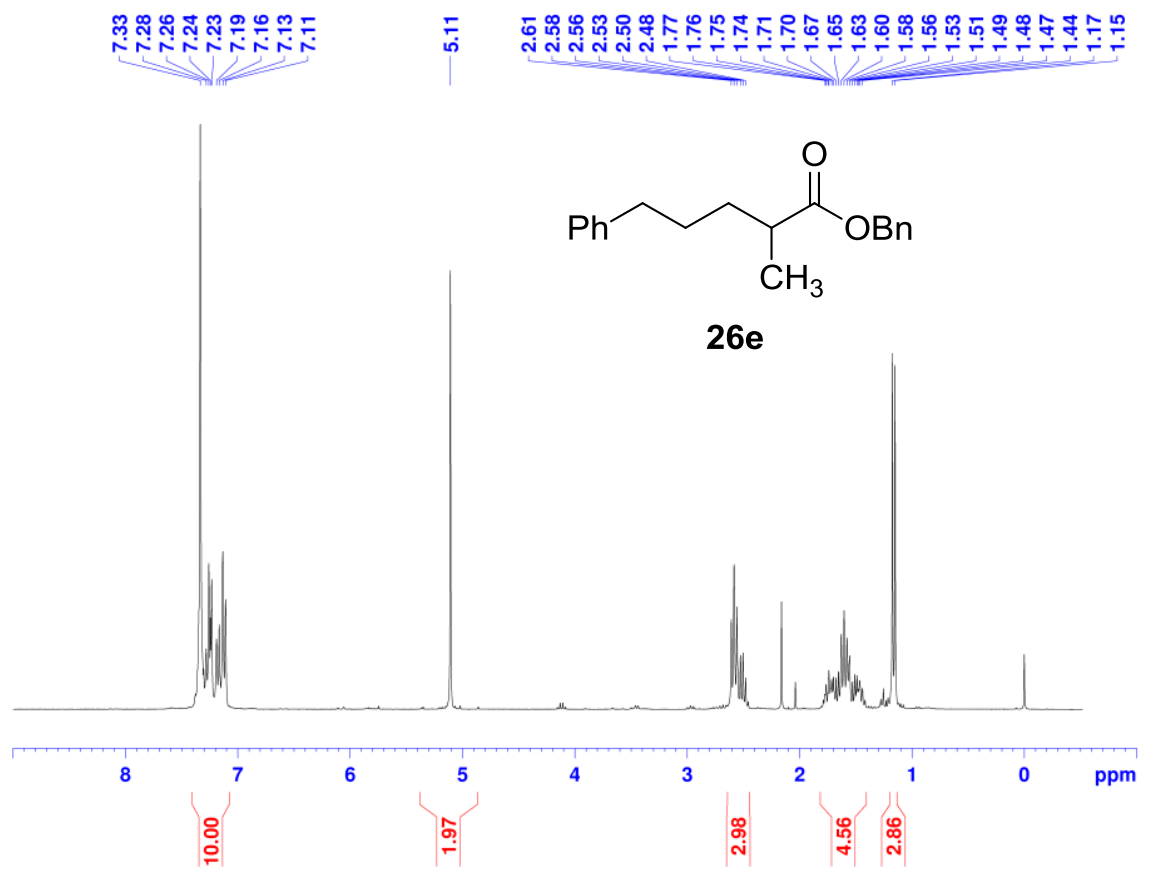

Figure $S 106{ }^{13} \mathrm{C}(600 \mathrm{MHz}) \mathrm{NMR}$ spectrum of $26 \mathrm{e}\left(\mathrm{CDCl}_{3}, 298 \mathrm{~K}\right)$.

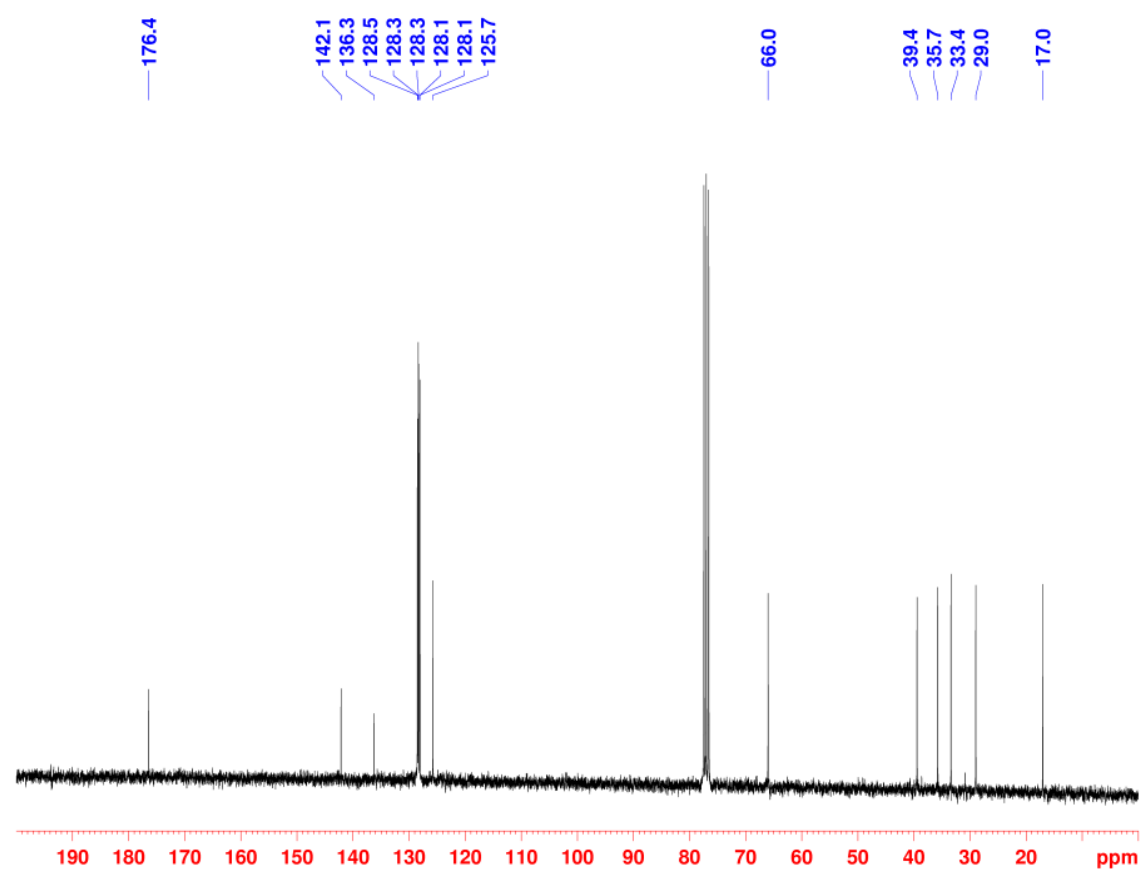

Figure $S 107{ }^{13} \mathrm{C}(150 \mathrm{MHz}) \mathrm{NMR}$ spectrum of $26 \mathrm{e}\left(\mathrm{CDCl}_{3}, 298 \mathrm{~K}\right)$. 


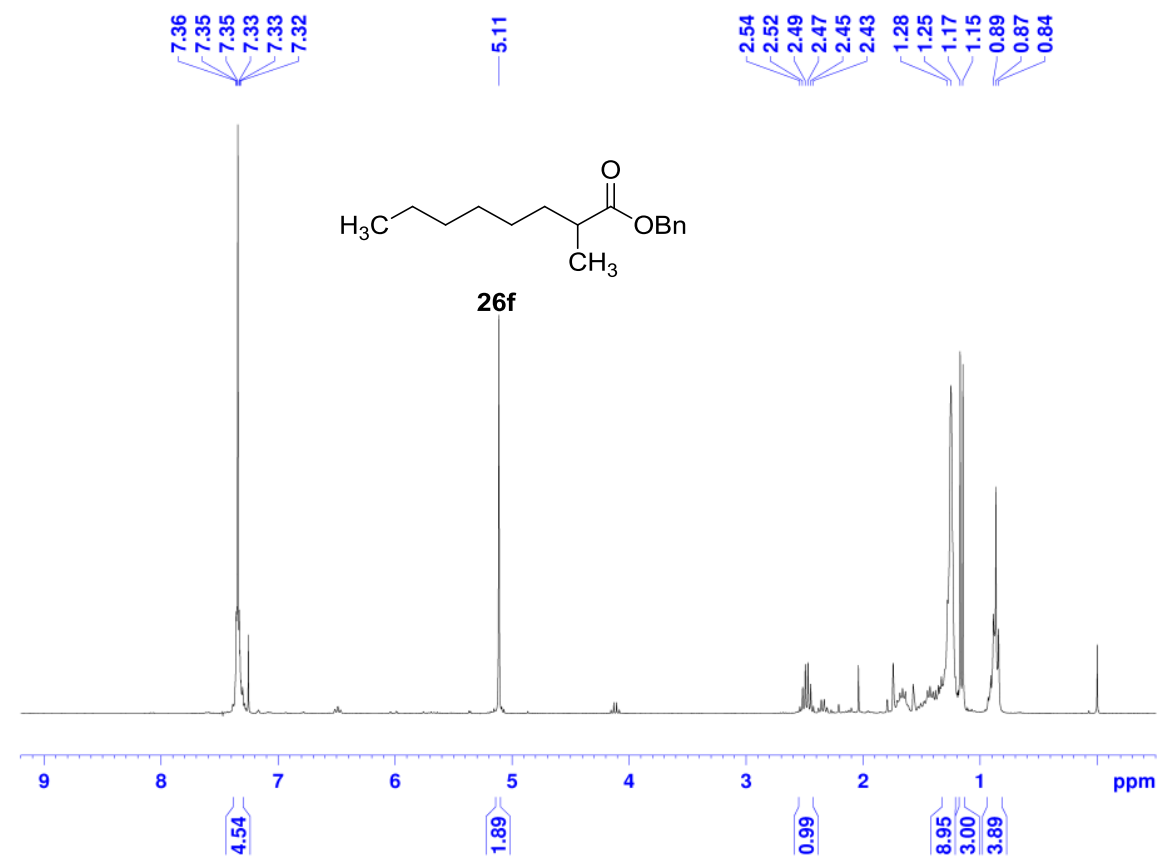

Figure $S 108{ }^{1} \mathrm{H}(600 \mathrm{MHz}) \mathrm{NMR}$ spectrum of $26 f\left(\mathrm{CDCl}_{3}, 298 \mathrm{~K}\right)$.
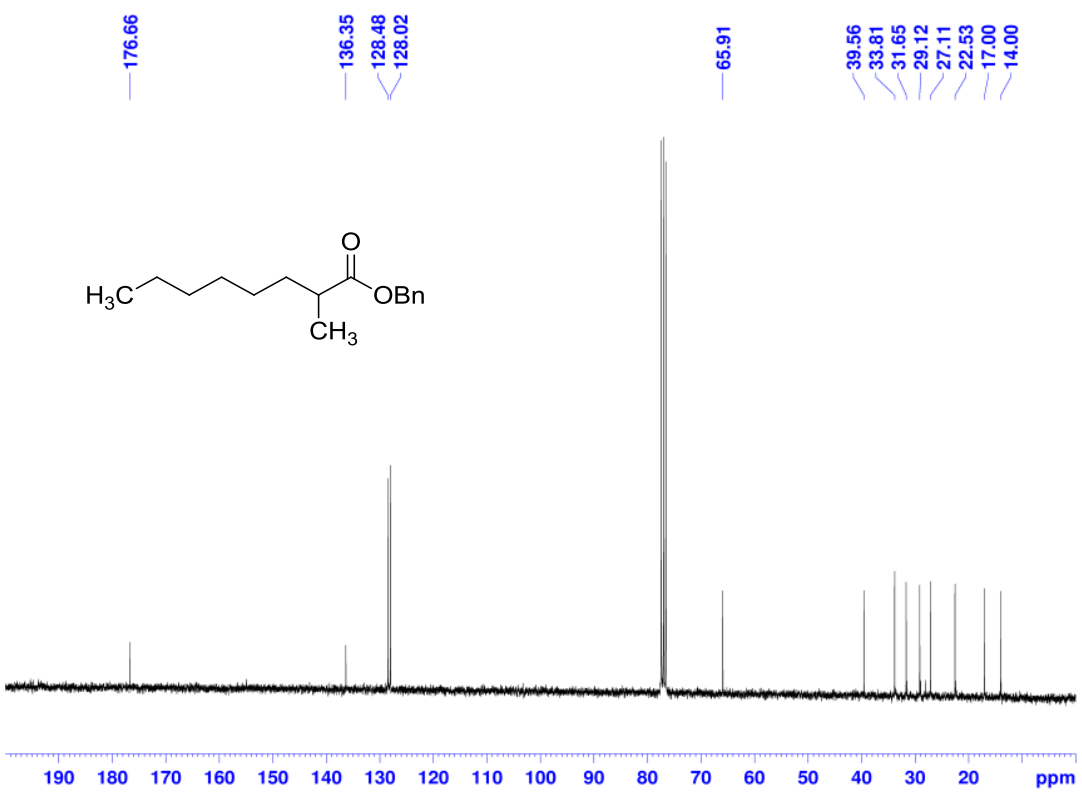

Figure $\mathbf{S 1 0 9}{ }^{13} \mathrm{C}(150 \mathrm{MHz}) \mathrm{NMR}$ spectrum of $26 f\left(\mathrm{CDCl}_{3}, 298 \mathrm{~K}\right)$. 


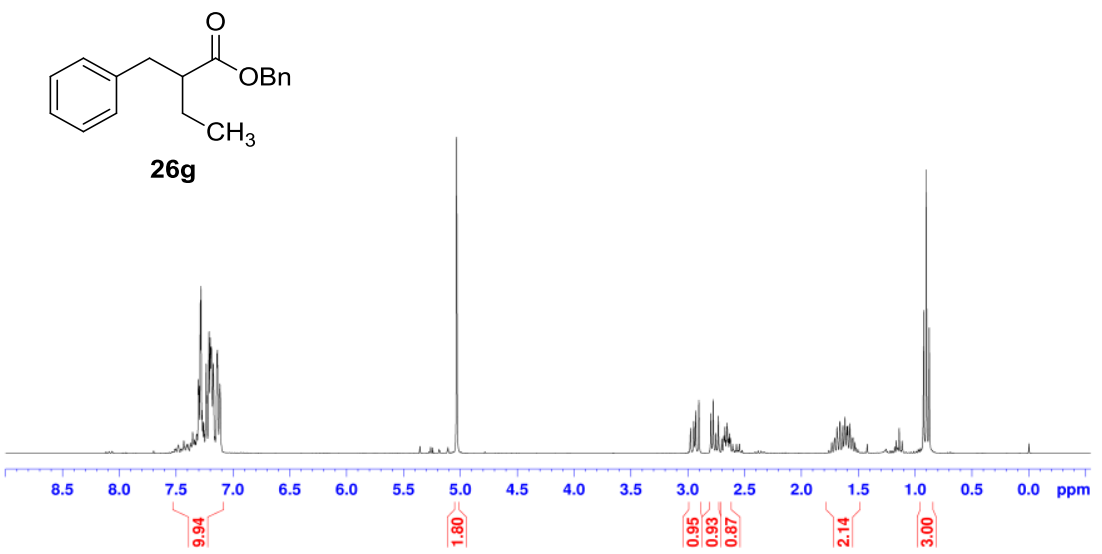

Figure $\mathbf{S 1 1 0}{ }^{1} \mathrm{H}(600 \mathrm{MHz}) \mathrm{NMR}$ spectrum of $\mathbf{2 6 g}\left(\mathrm{CDCl}_{3}, 298 \mathrm{~K}\right)$.

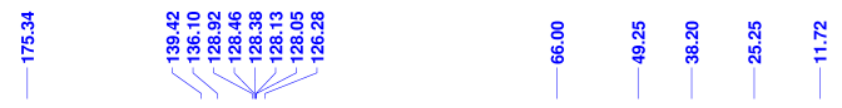<smiles>CCCCCCOC(=O)C(CC)Cc1ccccc1</smiles>

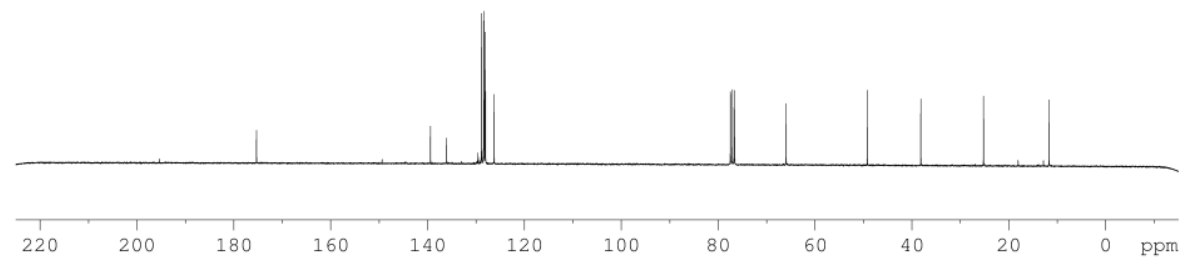

Figure $\mathbf{S 1 1 1}{ }^{13} \mathrm{C}(150 \mathrm{MHz}) \mathrm{NMR}$ spectrum of $\mathbf{2 6 g}\left(\mathrm{CDCl}_{3}, 298 \mathrm{~K}\right)$. 


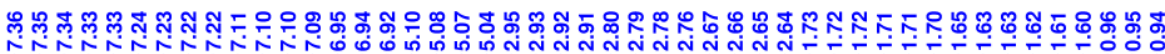<smiles>CCC(Cc1ccc(F)cc1)C(=O)Br</smiles>

$26 h$

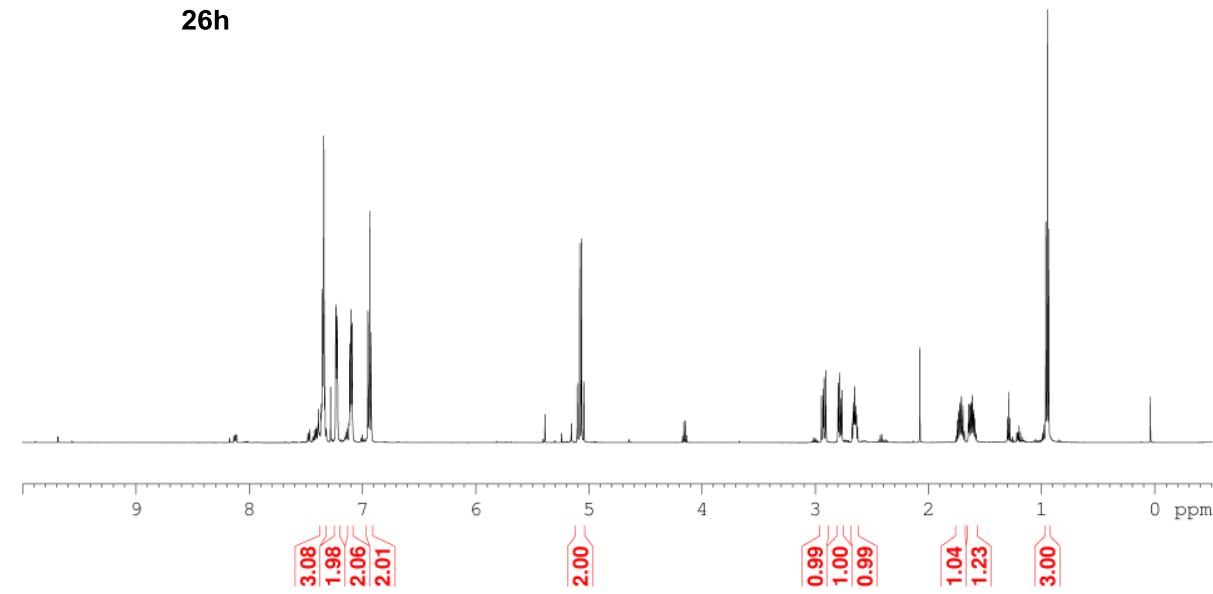

Figure $\mathbf{S 1 1 2}{ }^{1} \mathrm{H}(600 \mathrm{MHz}) \mathrm{NMR}$ spectrum of $26 \mathrm{~h}\left(\mathrm{CDCl}_{3}, 298 \mathrm{~K}\right)$.

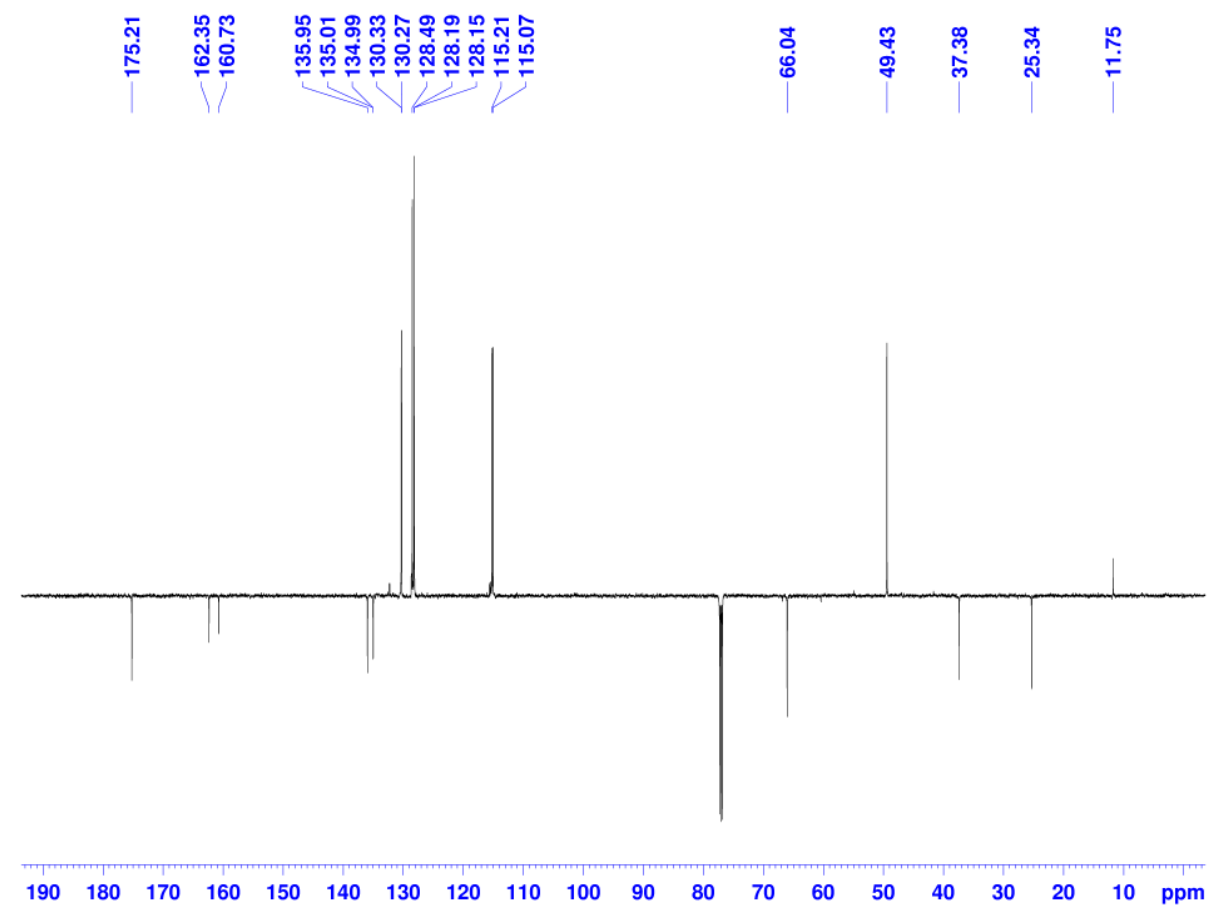

Figure $\mathbf{S 1 1 3}{ }^{13} \mathrm{C}(150 \mathrm{MHz})$ DEPTQ NMR spectrum of $26 \mathrm{~h}\left(\mathrm{CDCl}_{3}, 298 \mathrm{~K}\right)$. 


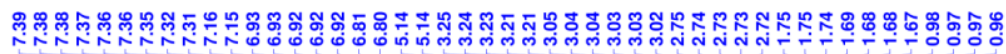

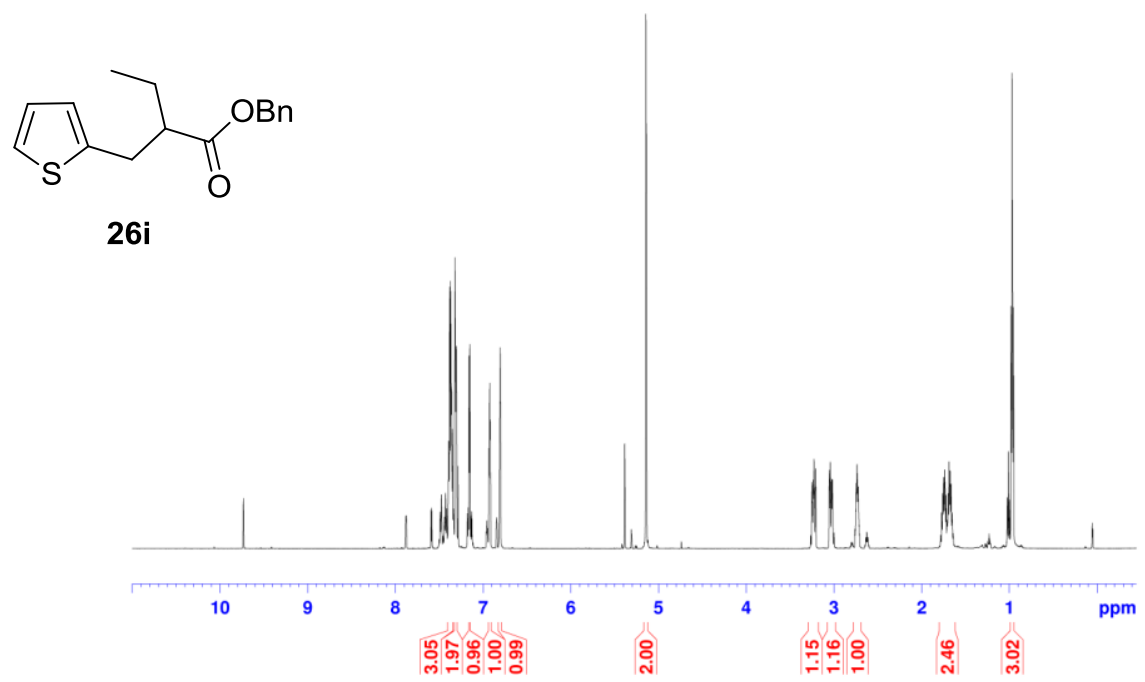

Figure $5114{ }^{1} \mathrm{H}(600 \mathrm{MHz}) \mathrm{NMR}$ spectrum of $26 \mathbf{i}$ (containing impurities presumably saturated aldehyde, unsaturated ester) $\left(\mathrm{CDCl}_{3}, 298 \mathrm{~K}\right)$.

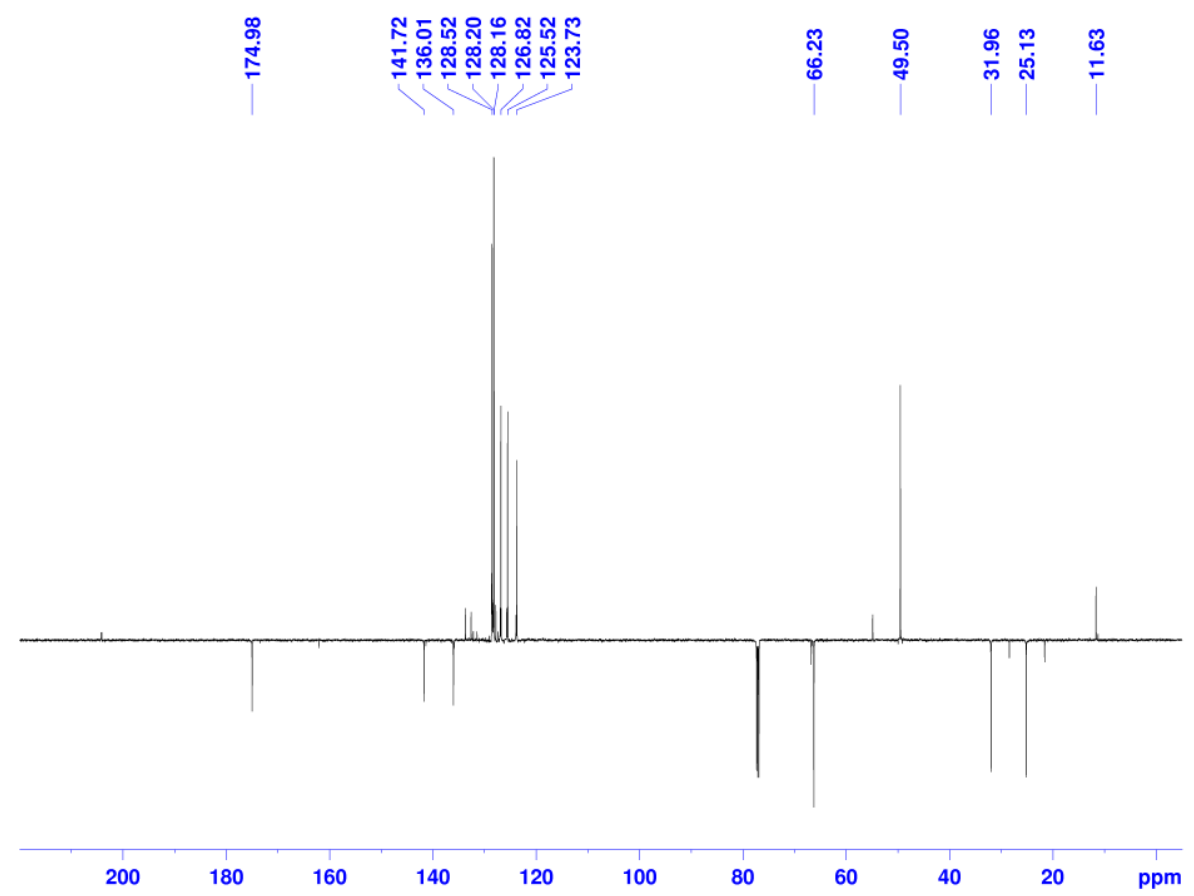

Figure $\mathbf{S 1 1 5}{ }^{13} \mathrm{C}(150 \mathrm{MHz})$ DEPTQ NMR spectrum of 26i (containing impurities presumably saturated aldehyde, unsaturated ester) $\left(\mathrm{CDCl}_{3}, 298 \mathrm{~K}\right)$. 


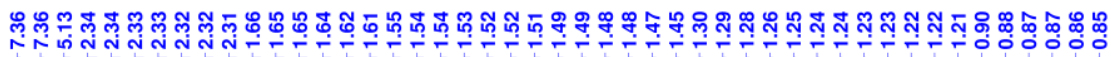

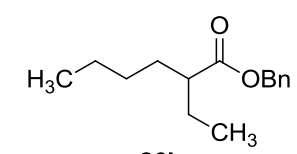

26j

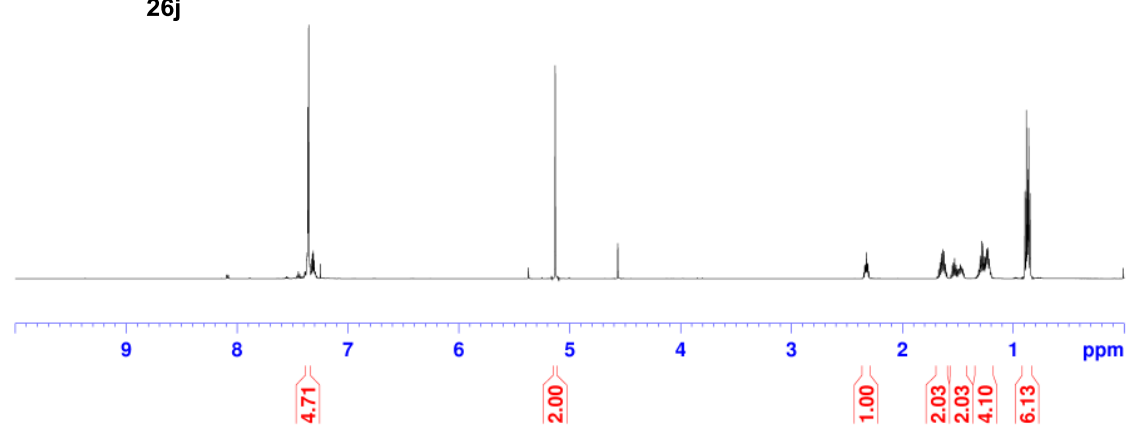

Figure $S 116{ }^{1} \mathrm{H}(600 \mathrm{MHz}) \mathrm{NMR}$ spectrum of 26j (containing impurity benzyl alcohol) ( $\mathrm{CDCl}_{3}$, 298 K).

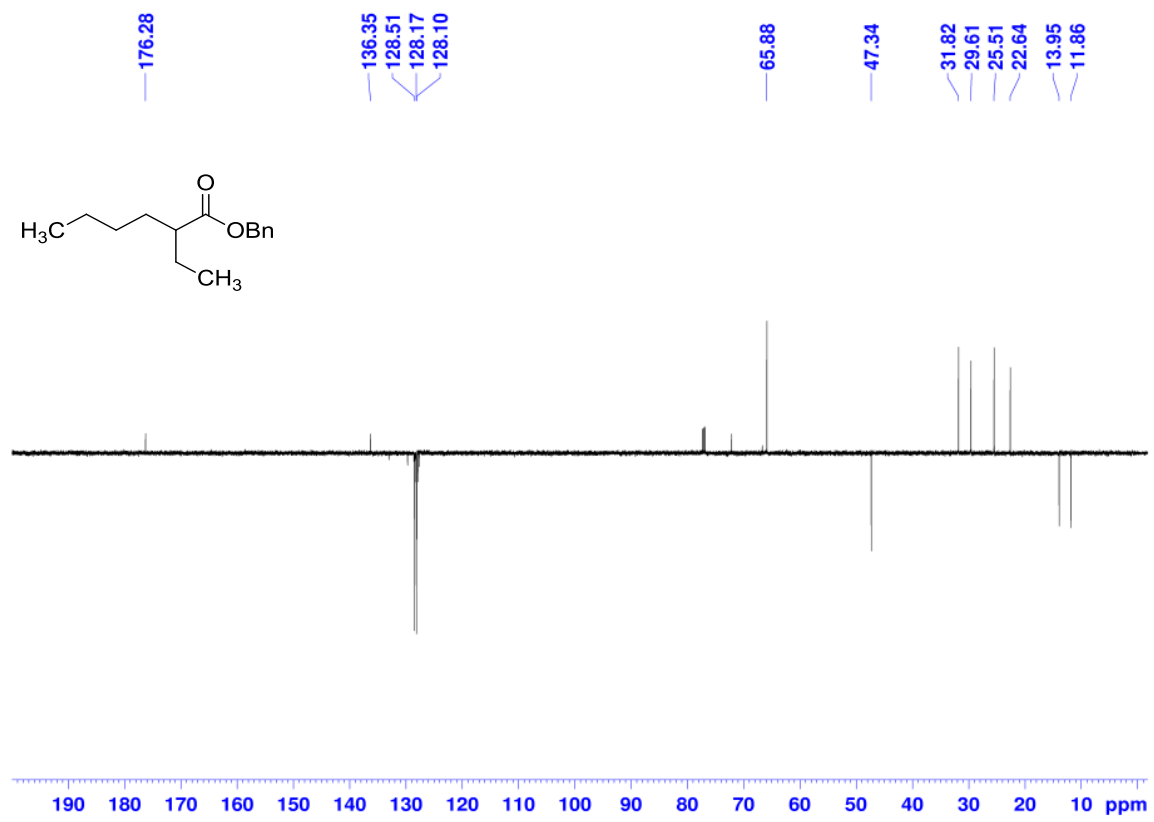

Figure $\mathbf{S 1 1 7}{ }^{13} \mathrm{C}(150 \mathrm{MHz})$ DEPTQ NMR spectrum of 26j (containing impurity benzyl alcohol) $\left(\mathrm{CDCl}_{3}, 298 \mathrm{~K}\right)$. 
<smiles>CC(C)C(Cc1ccco1)C(=O)OCc1ccccc1</smiles>

26k

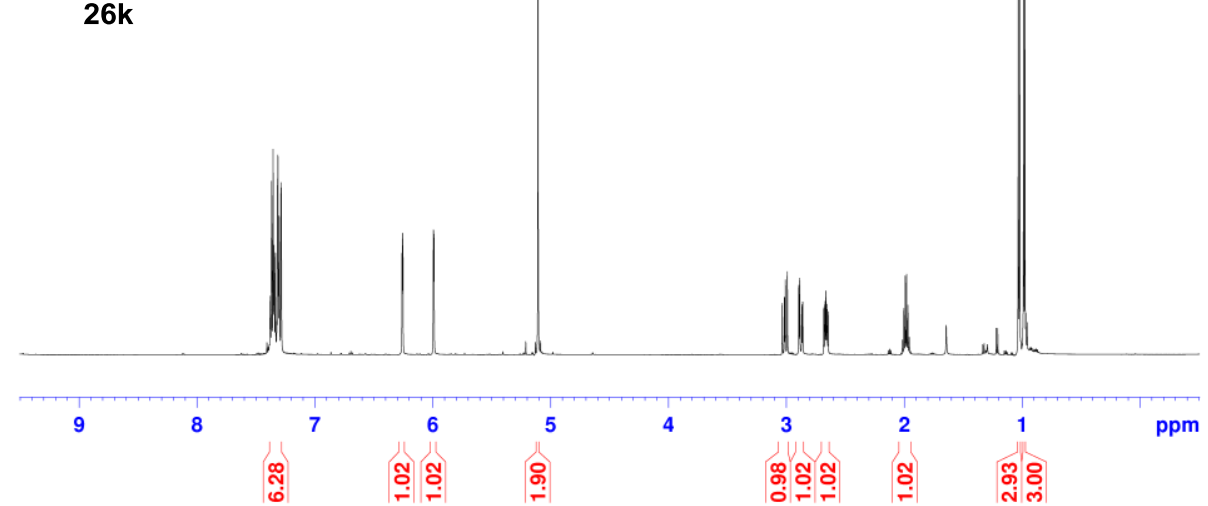

Figure $\mathbf{S 1 1 8}{ }^{1} \mathrm{H}(600 \mathrm{MHz}) \mathrm{NMR}$ spectrum of $\mathbf{2 6 k}\left(\mathrm{CDCl}_{3}, 298 \mathrm{~K}\right)$.

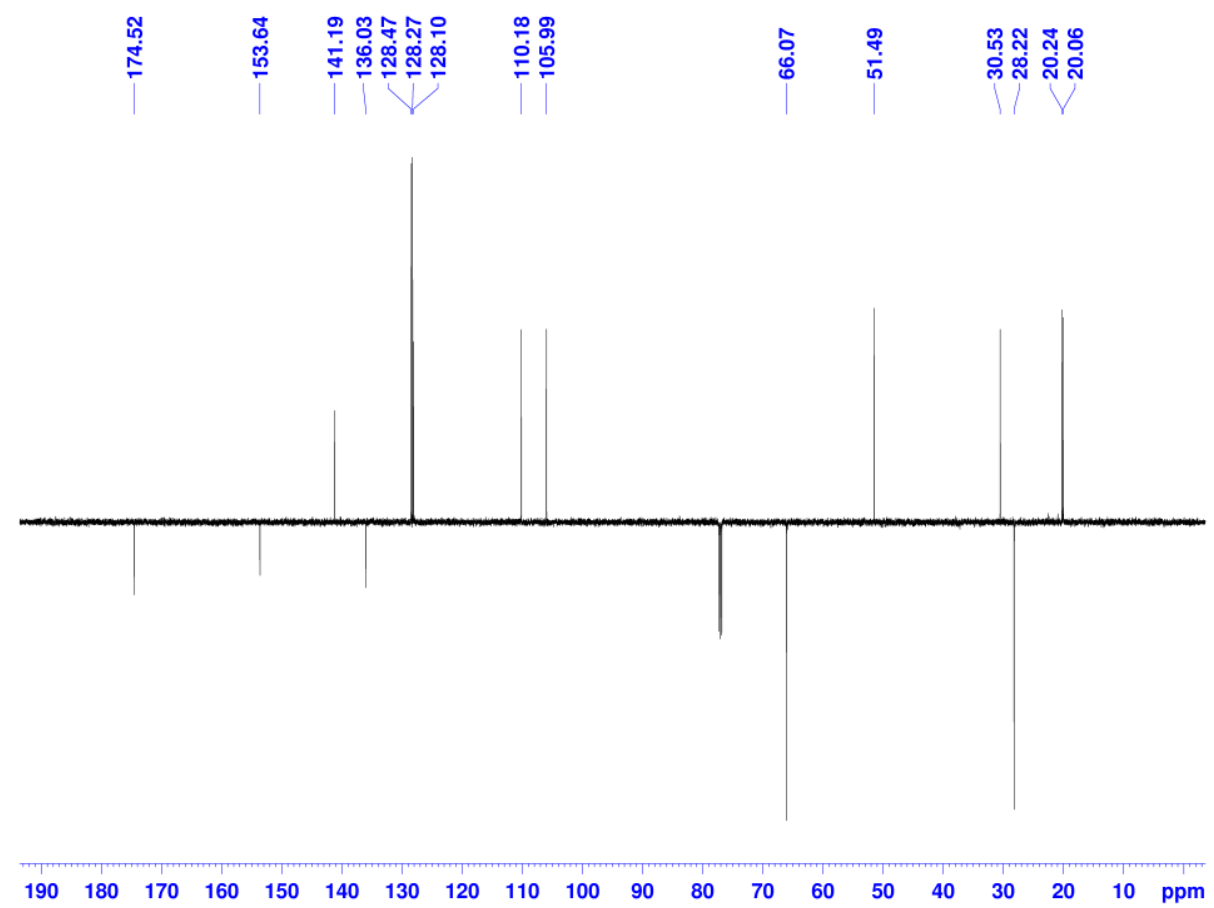

Figure $\mathbf{S 1 1 9}{ }^{13} \mathrm{C}(150 \mathrm{MHz})$ DEPTQ NMR spectrum of $\mathbf{2 6 k}\left(\mathrm{CDCl}_{3}, 298 \mathrm{~K}\right)$. 


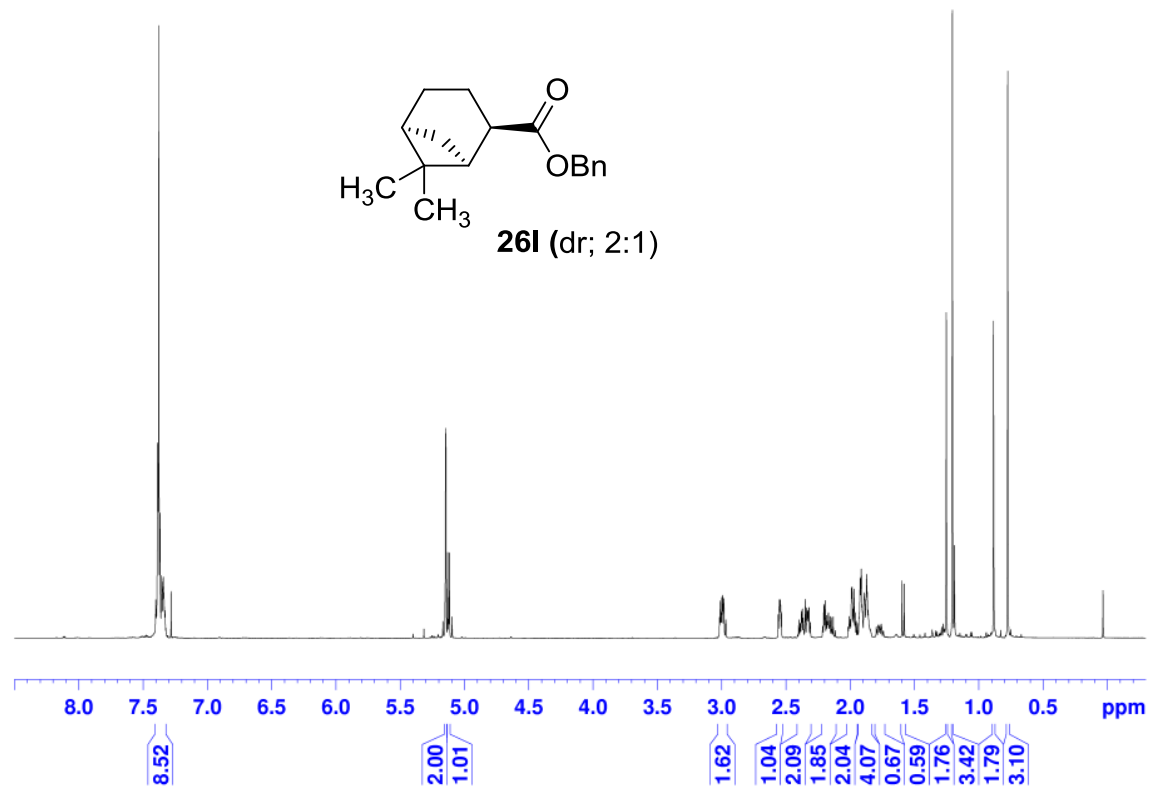

Figure $5120^{1} \mathrm{H}(600 \mathrm{MHz}) \mathrm{NMR}$ spectrum of $26 \mathrm{I}\left(\mathrm{CDCl}_{3}, 298 \mathrm{~K}\right)$.
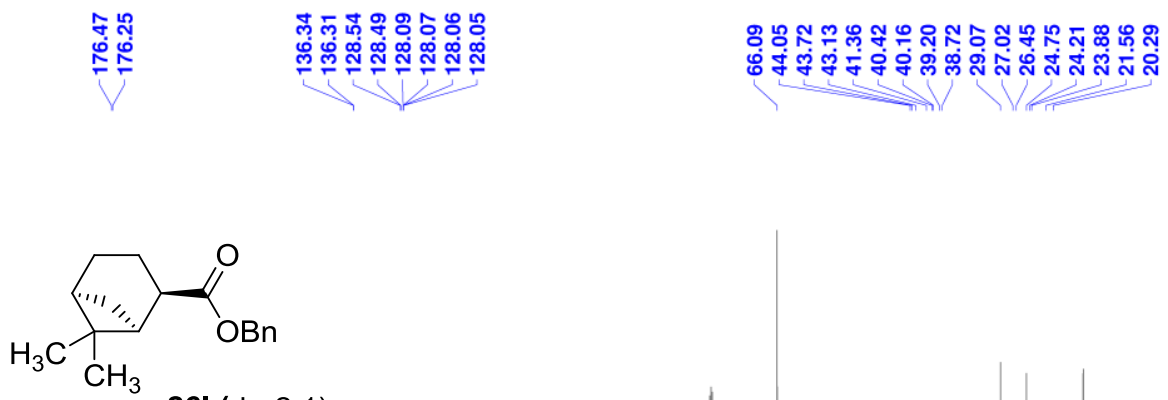

$26 I(d r ; 2: 1)$

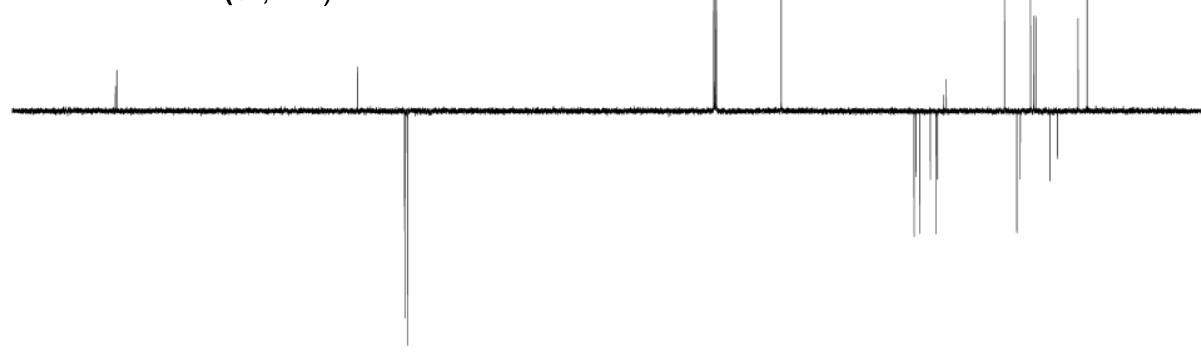

$\begin{array}{llllllllllllllllllll}190 & 180 & 170 & 160 & 150 & 140 & 130 & 120 & 110 & 100 & 90 & 80 & 70 & 60 & 50 & 40 & 30 & 20 & 10 & \mathrm{ppm}\end{array}$

Figure $\mathbf{S 1 2 1}{ }^{13} \mathrm{C}(150 \mathrm{MHz})$ DEPTQ NMR spectrum of $26 \mathbf{I}\left(\mathrm{CDCl}_{3}, 298 \mathrm{~K}\right)$. 


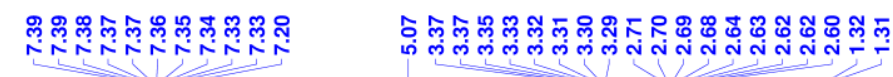<smiles>CCCOC(=O)CC(C)c1ccccc1</smiles>

26n

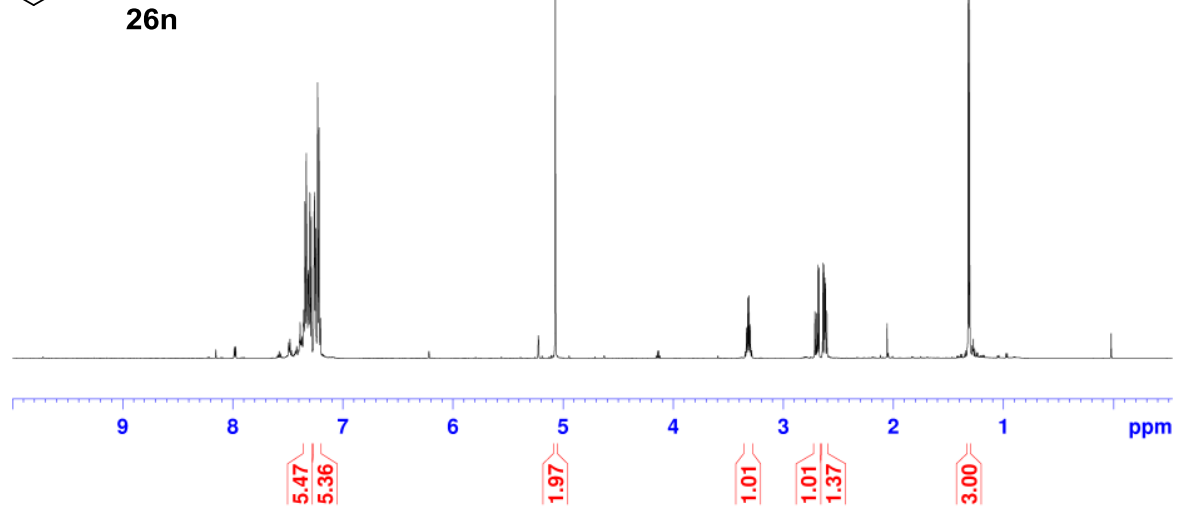

Figure $S 122{ }^{1} \mathrm{H}(600 \mathrm{MHz}) \mathrm{NMR}$ spectrum of $26 \mathrm{n}\left(\mathrm{CDCl}_{3}, 298 \mathrm{~K}\right)$.

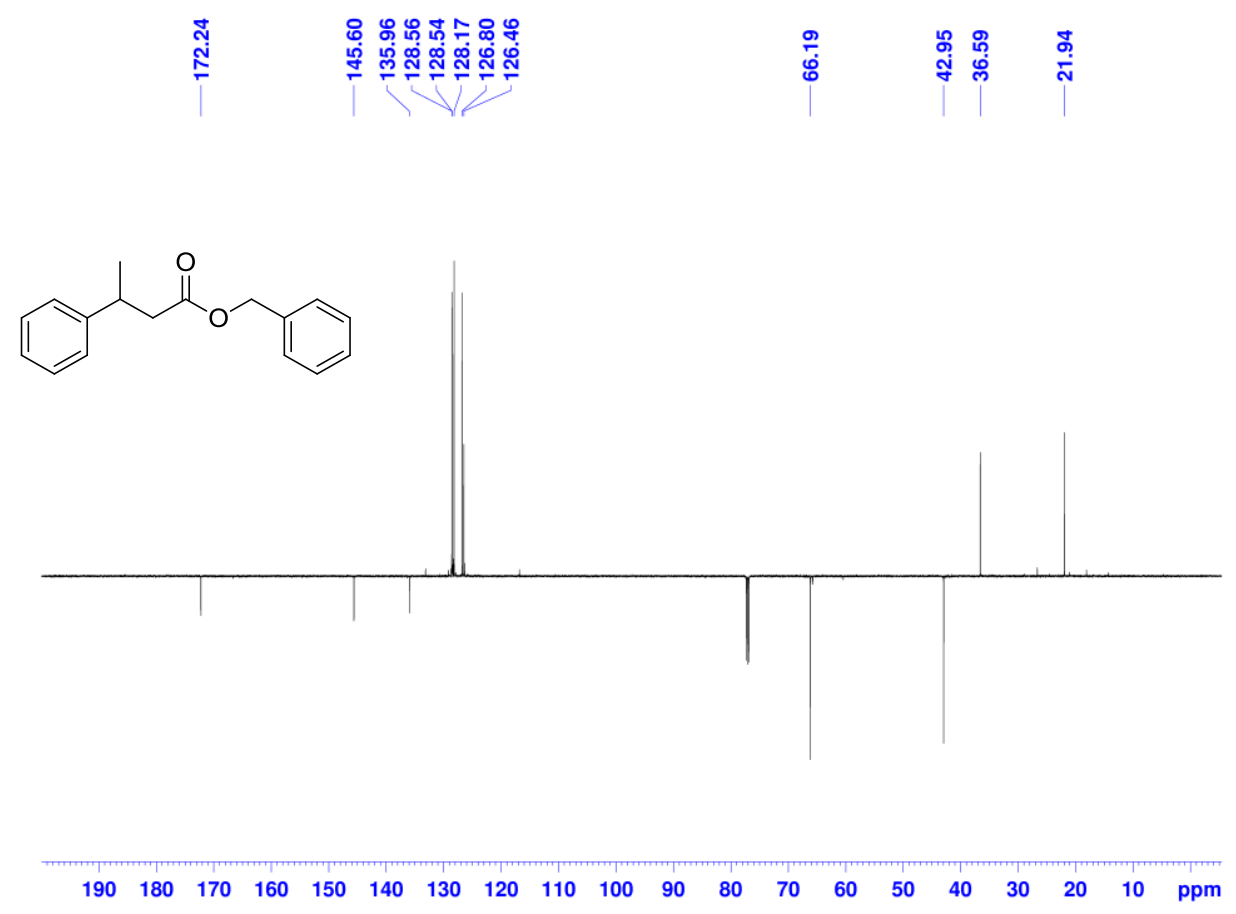

Figure $\mathbf{S 1 2 3}{ }^{13} \mathrm{C}(150 \mathrm{MHz})$ DEPTQ NMR spectrum of $26 \mathrm{n}\left(\mathrm{CDCl}_{3}, 298 \mathrm{~K}\right)$. 


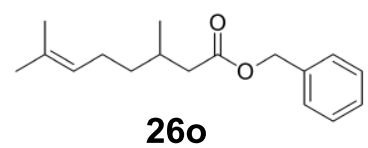

260

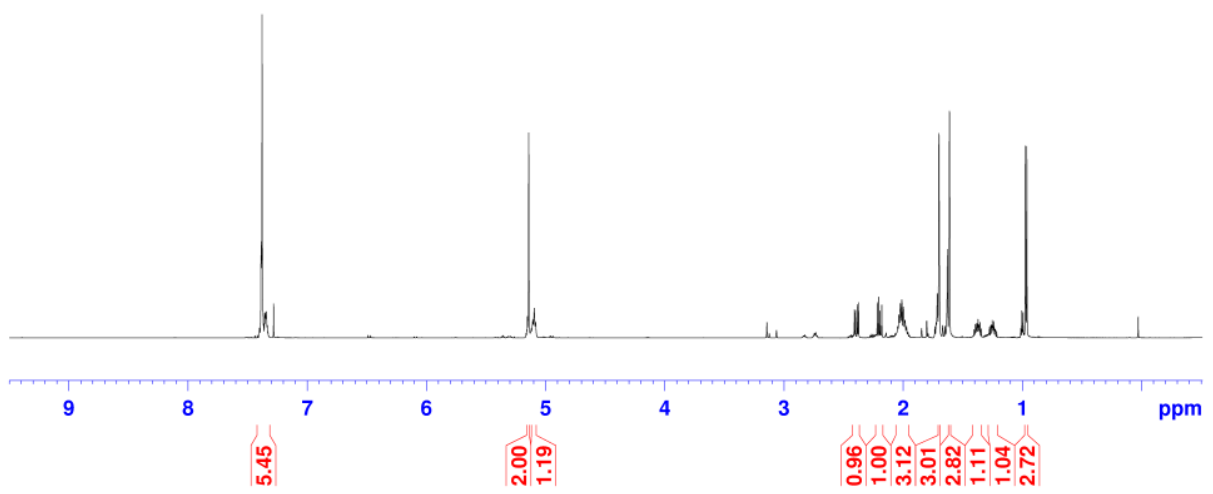

Figure $S 124{ }^{1} \mathrm{H}(600 \mathrm{MHz}) \mathrm{NMR}$ spectrum of $260\left(\mathrm{CDCl}_{3}, 298 \mathrm{~K}\right)$.

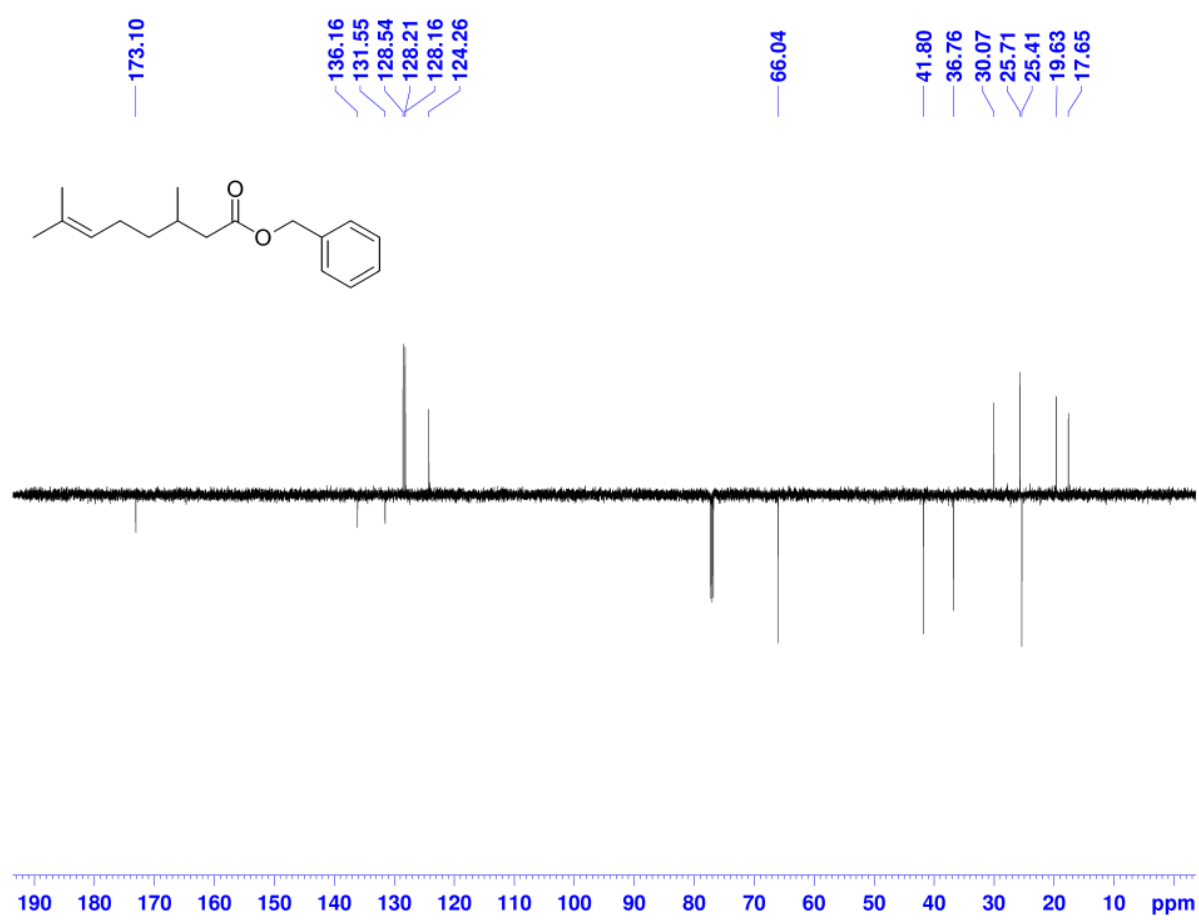

Figure $S 125{ }^{13} \mathrm{C}(150 \mathrm{MHz})$ DEPTQ NMR spectrum of $260\left(\mathrm{CDCl}_{3}, 298 \mathrm{~K}\right)$. 


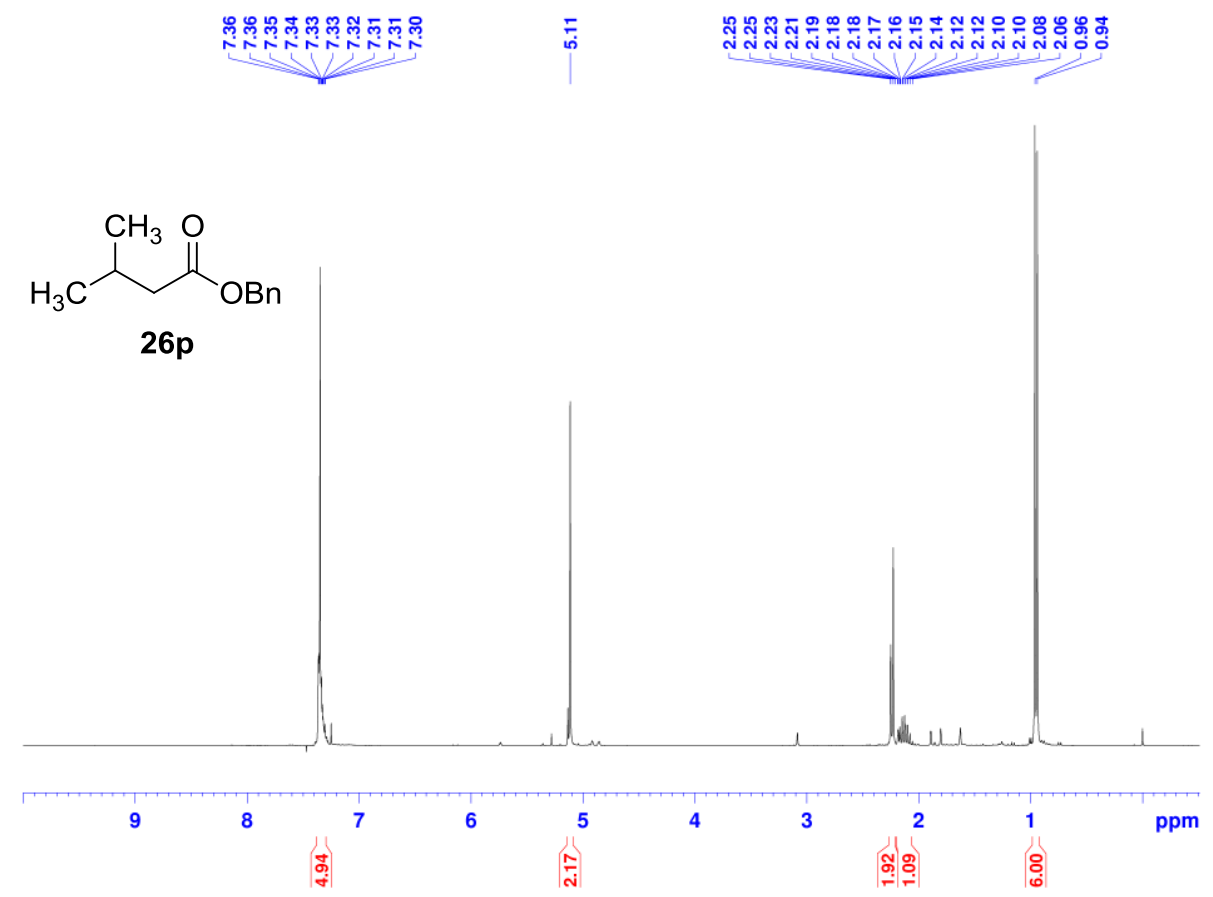

Figure $S 126{ }^{1} \mathrm{H}(600 \mathrm{MHz}) \mathrm{NMR}$ spectrum of $26 \mathrm{p}\left(\mathrm{CDCl}_{3}, 298 \mathrm{~K}\right)$.

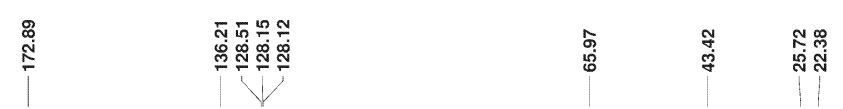

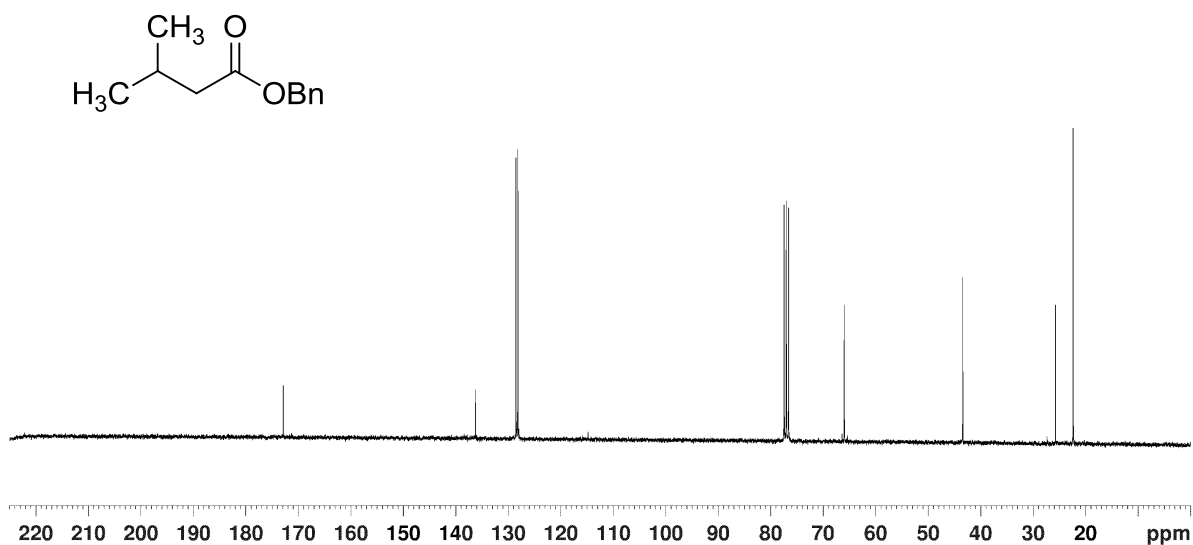

Figure $S 127{ }^{13} \mathrm{C}(150 \mathrm{MHz}) \mathrm{NMR}$ spectrum of 26p $\left(\mathrm{CDCl}_{3}, 298 \mathrm{~K}\right)$. 


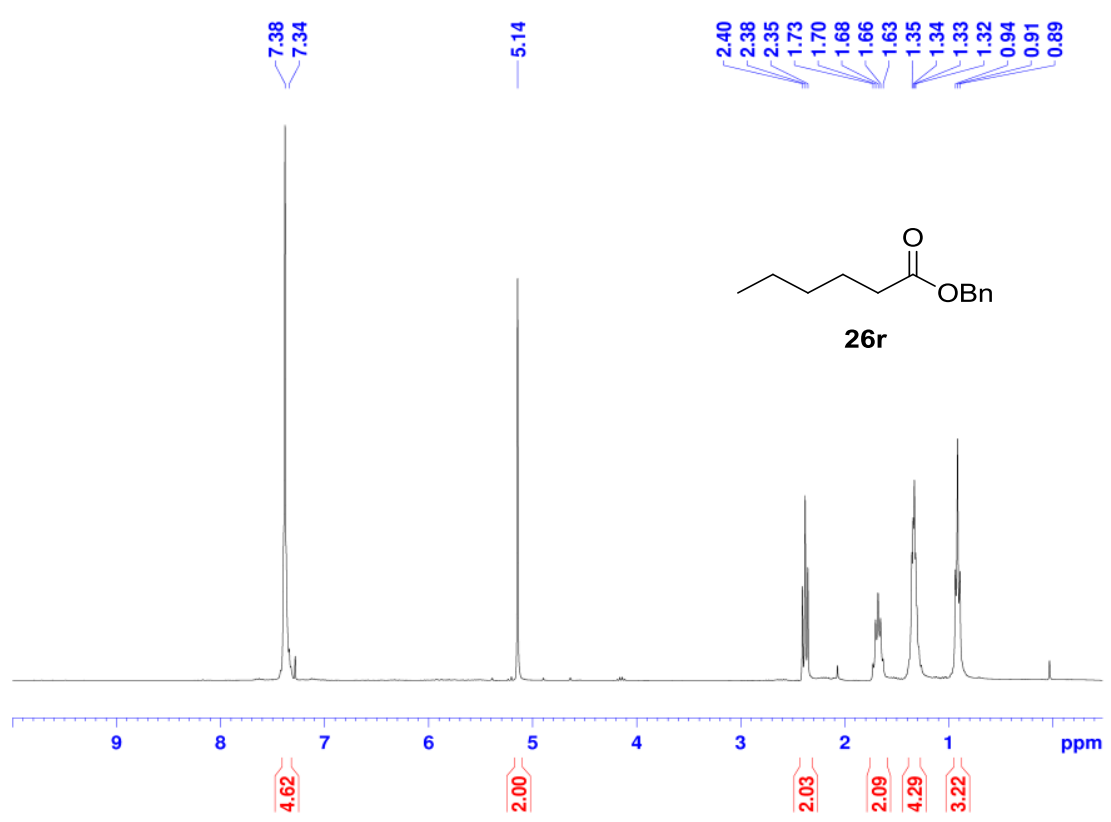

Figure $S 128{ }^{1} \mathrm{H}(600 \mathrm{MHz}) \mathrm{NMR}$ spectrum of $26 \mathrm{r}\left(\mathrm{CDCl}_{3}, 298 \mathrm{~K}\right)$.
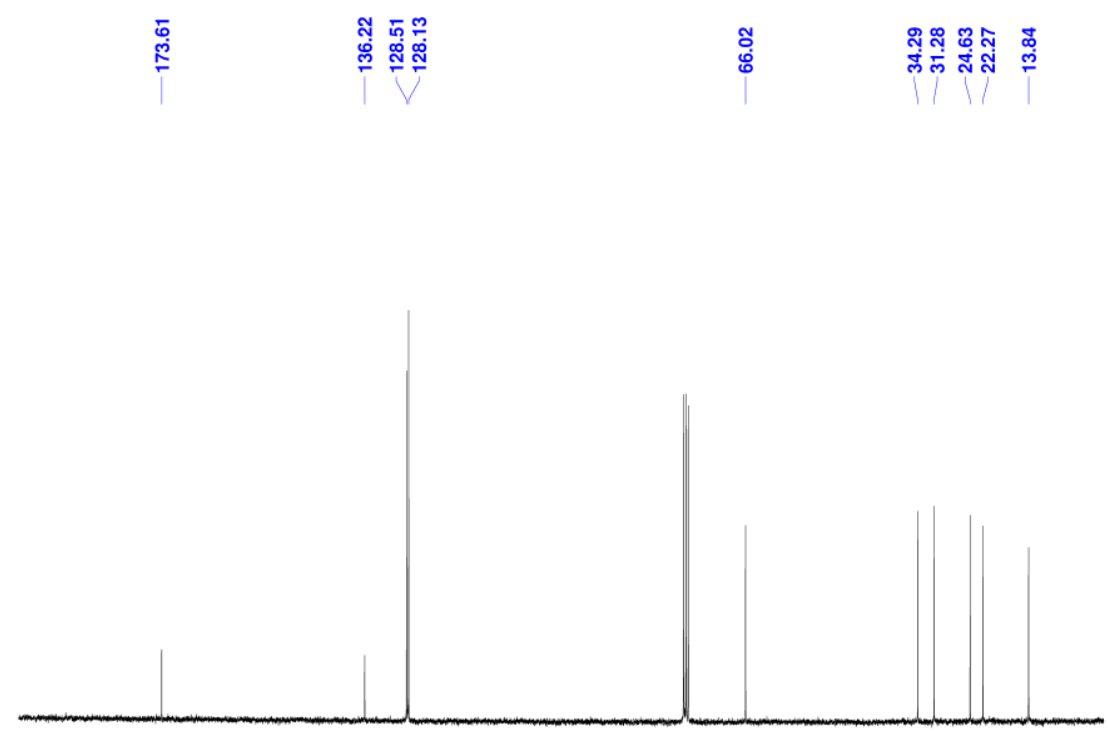

$\begin{array}{lllllllllllllllllll}190 & 180 & 170 & 160 & 150 & 140 & 130 & 120 & 110 & 100 & 90 & 80 & 70 & 60 & 50 & 40 & 30 & 20 & \mathrm{ppm}\end{array}$

Figure $S 129{ }^{13} \mathrm{C}(150 \mathrm{MHz}) \mathrm{NMR}$ spectrum of $26 \mathbf{r}\left(\mathrm{CDCl}_{3}, 298 \mathrm{~K}\right)$. 


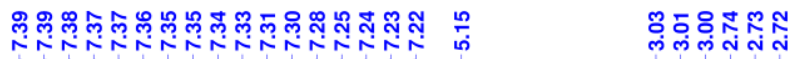

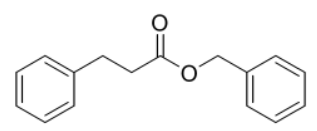

26s

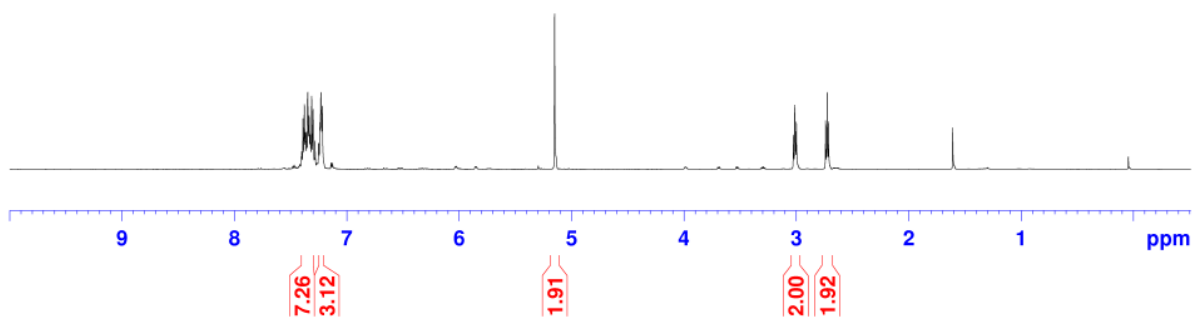

Figure $5130^{1} \mathrm{H}(600 \mathrm{MHz}) \mathrm{NMR}$ spectrum of $26 \mathrm{~s}\left(\mathrm{CDCl}_{3}, 298 \mathrm{~K}\right)$.

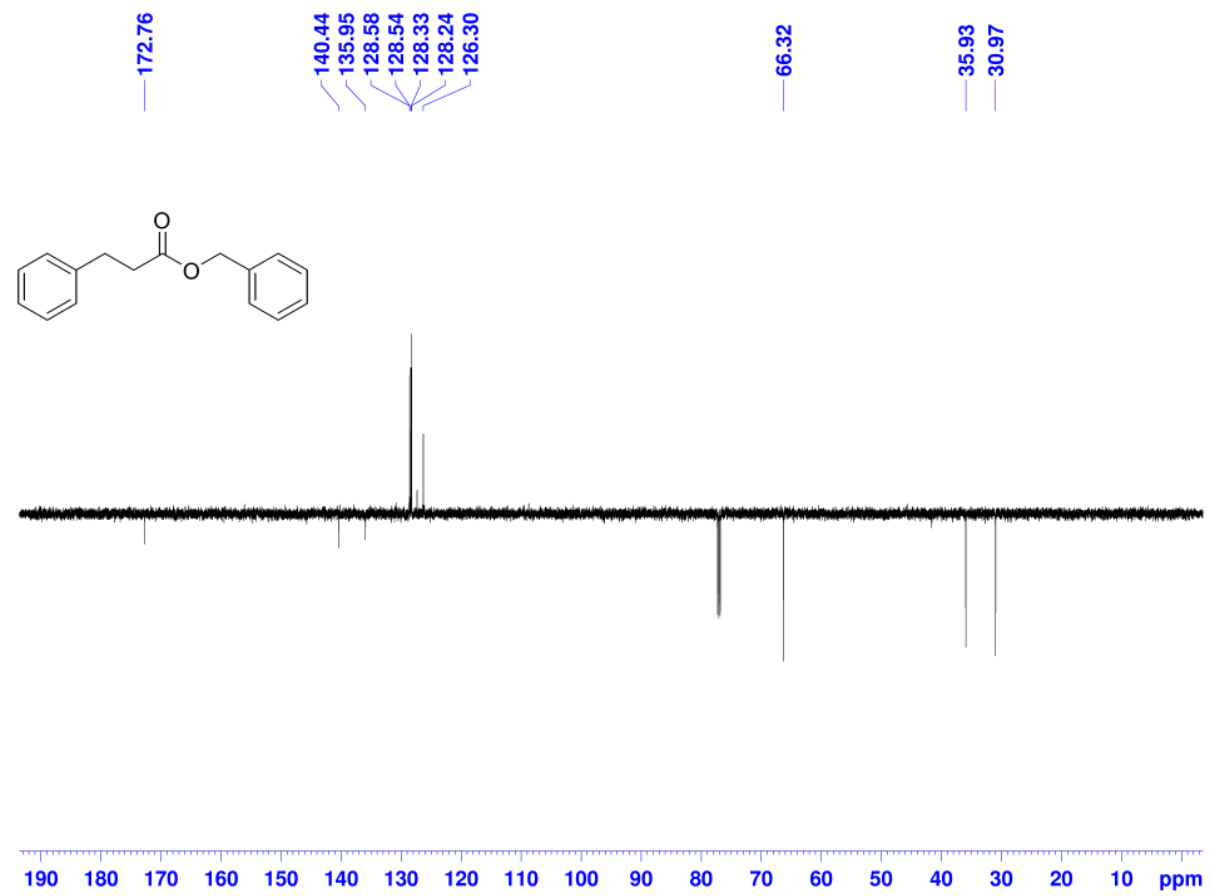

Figure $\mathbf{S 1 3 1}{ }^{13} \mathrm{C}(150 \mathrm{MHz})$ DEPTQ NMR spectrum of 26s $\left(\mathrm{CDCl}_{3}, 298 \mathrm{~K}\right)$. 


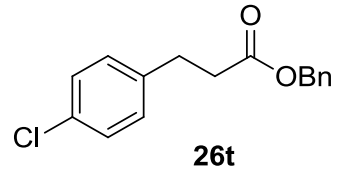

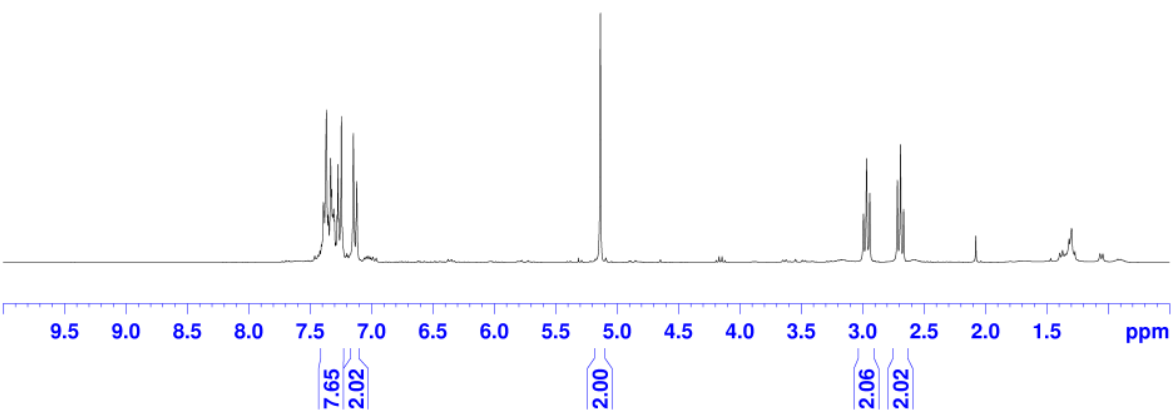

Figure $5132{ }^{1} \mathrm{H}(600 \mathrm{MHz}) \mathrm{NMR}$ spectrum of $26 \mathrm{t}\left(\mathrm{CDCl}_{3}, 298 \mathrm{~K}\right)$.

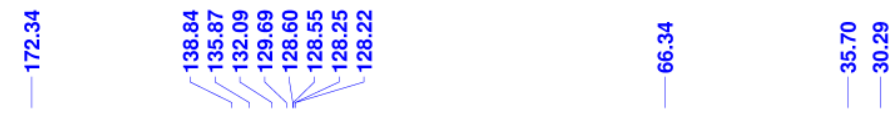

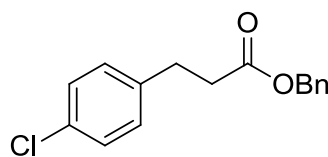

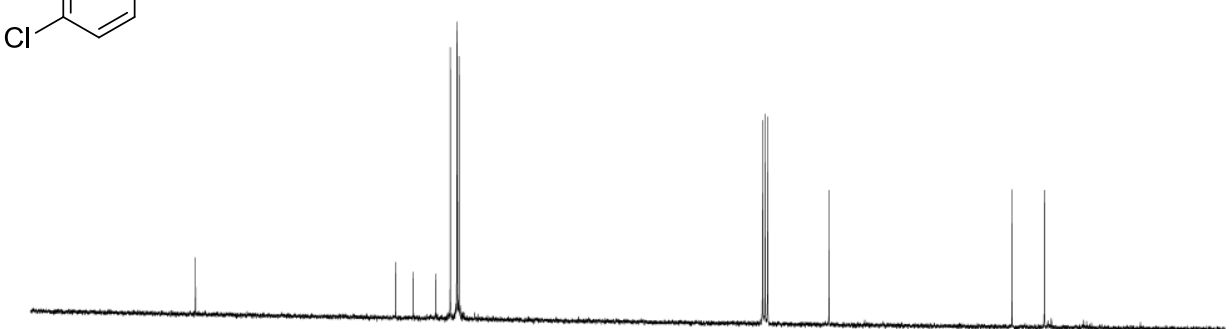

$\begin{array}{lllllllllllllllllll}190 & 180 & 170 & 160 & 150 & 140 & 130 & 120 & 110 & 100 & 90 & 80 & 70 & 60 & 50 & 40 & 30 & 20 & \mathrm{ppm}\end{array}$

Figure $\mathbf{S 1 3 3}{ }^{13} \mathrm{C}(150 \mathrm{MHz}) \mathrm{NMR}$ spectrum of $\mathbf{2 6 t}\left(\mathrm{CDCl}_{3}, 298 \mathrm{~K}\right)$. 


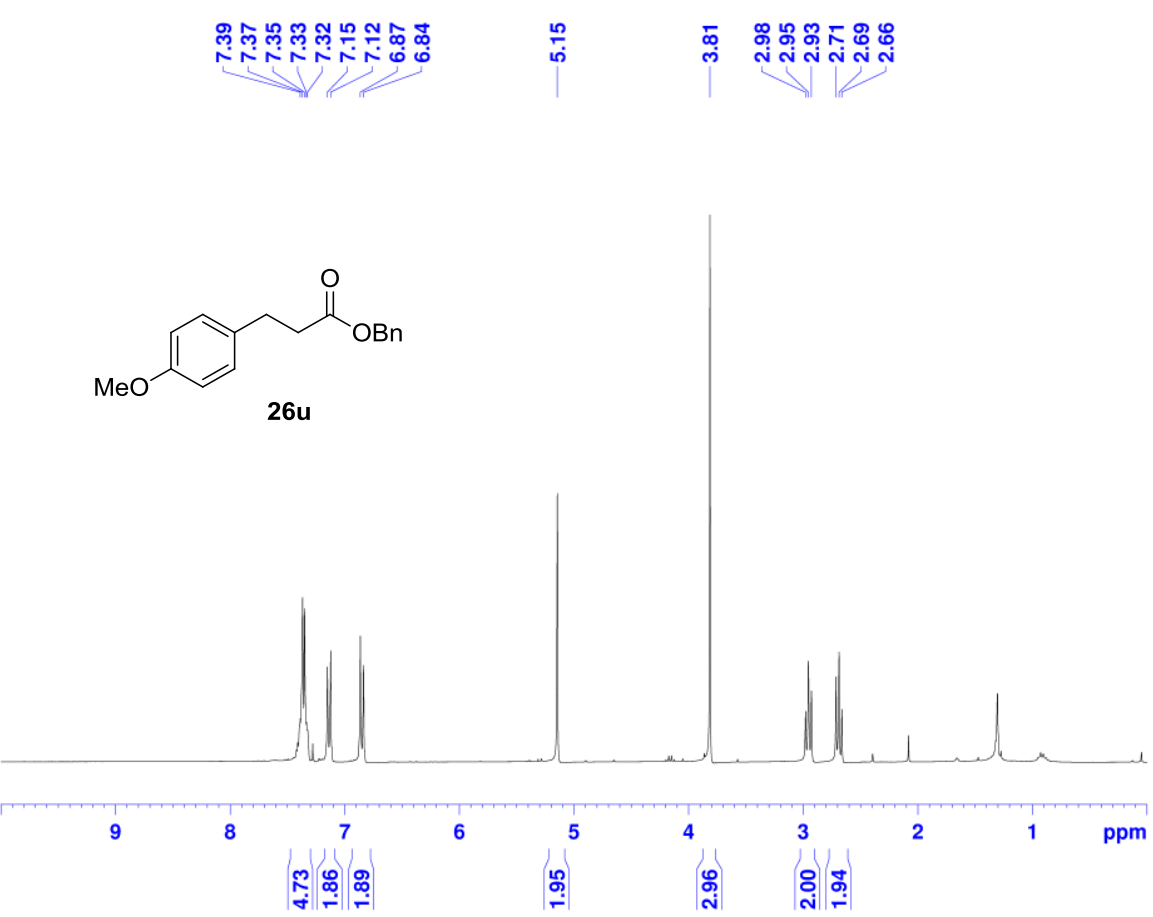

Figure $5134{ }^{1} \mathrm{H}(600 \mathrm{MHz}) \mathrm{NMR}$ spectrum of $\mathbf{2 6 u}\left(\mathrm{CDCl}_{3}, 298 \mathrm{~K}\right)$.
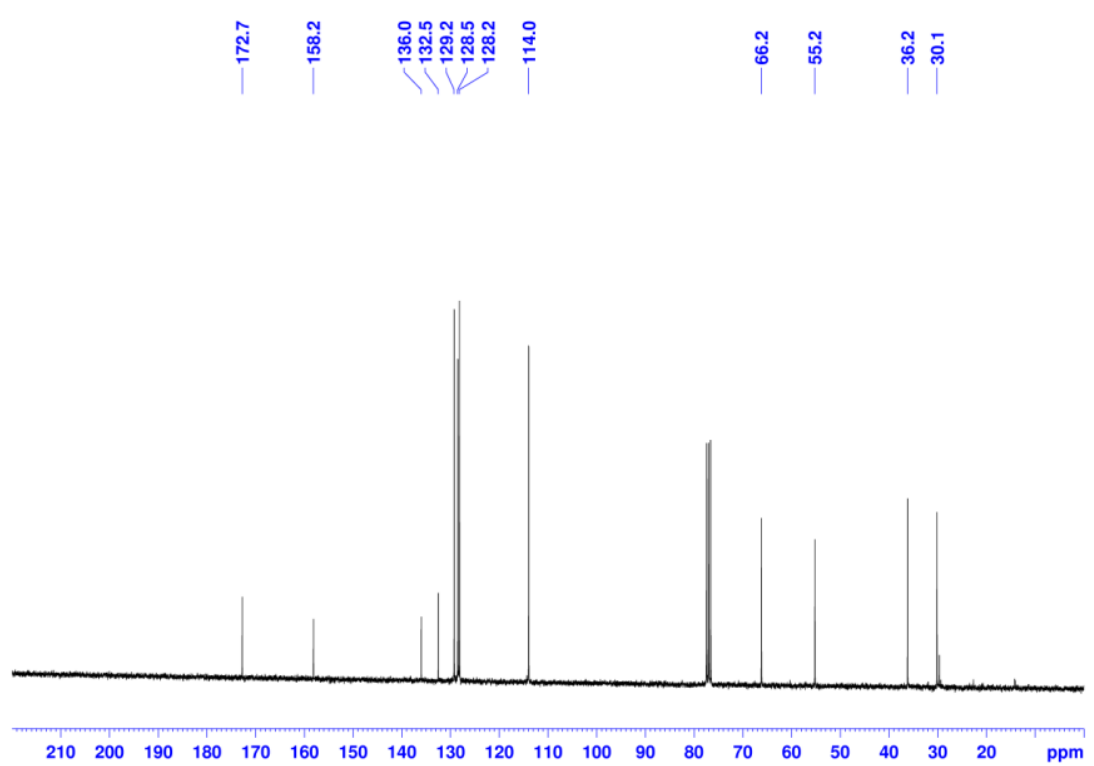

Figure $S 135{ }^{13} \mathrm{C}(150 \mathrm{MHz}) \mathrm{NMR}$ spectrum of $26 \mathrm{u}\left(\mathrm{CDCl}_{3}, 298 \mathrm{~K}\right)$. 


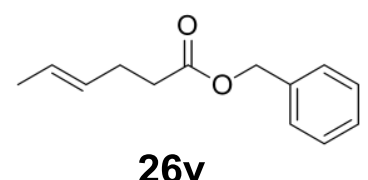

26v

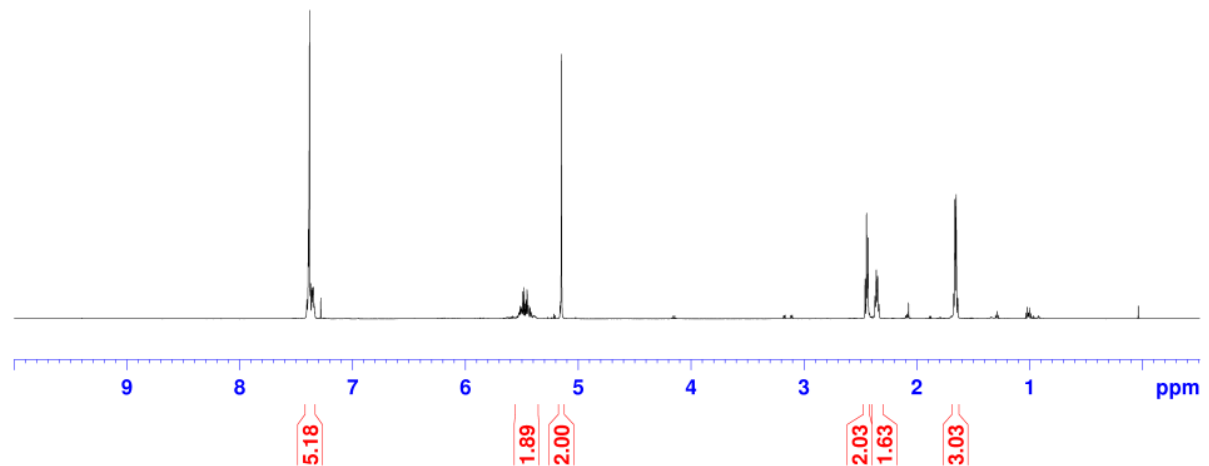

Figure $S 136{ }^{1} \mathrm{H}(600 \mathrm{MHz}) \mathrm{NMR}$ spectrum of $26 \mathbf{v}\left(\mathrm{CDCl}_{3}, 298 \mathrm{~K}\right)$.

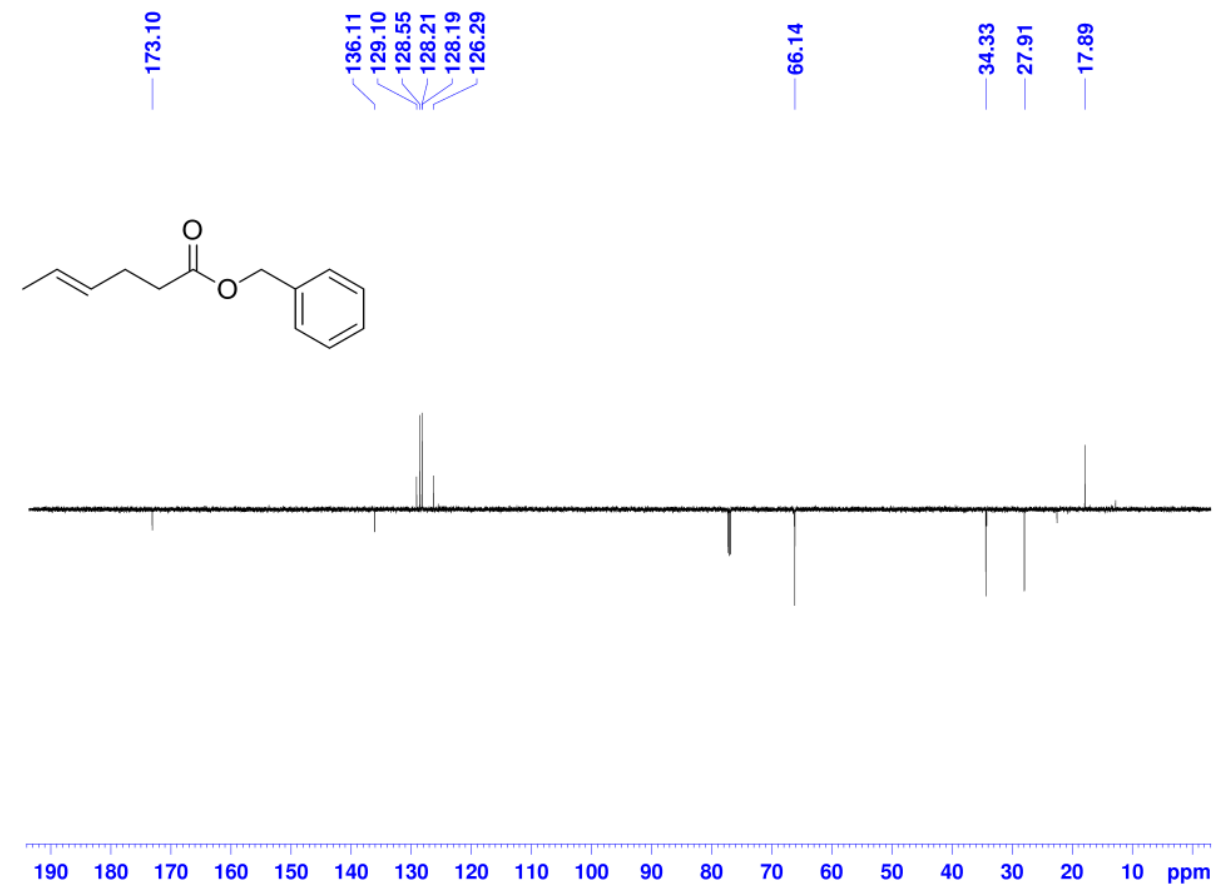

Figure $\mathbf{S 1 3 7}{ }^{13} \mathrm{C}(150 \mathrm{MHz})$ DEPTQ NMR spectrum of $\mathbf{2 6 v}\left(\mathrm{CDCl}_{3}, 298 \mathrm{~K}\right)$. 


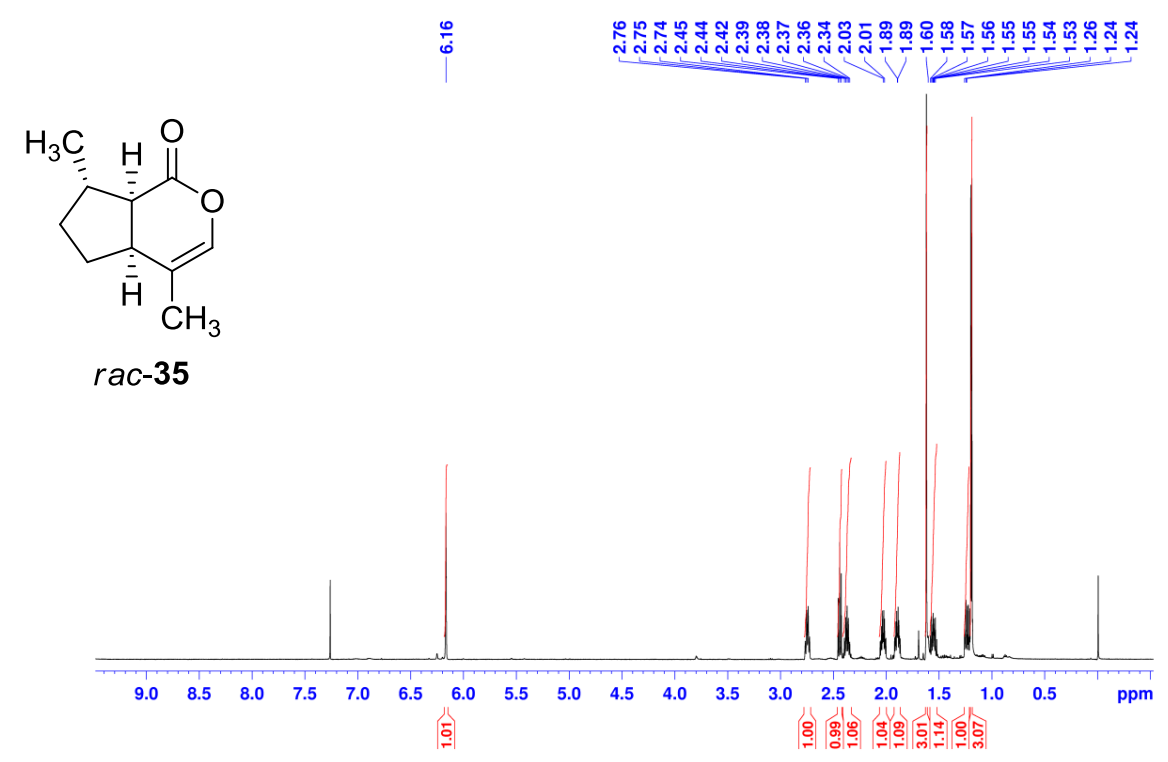

Figure $5138{ }^{13} \mathrm{C}(600 \mathrm{MHz}) \mathrm{NMR}$ spectrum of rac-35 $\left(\mathrm{CDCl}_{3}, 298 \mathrm{~K}\right)$.

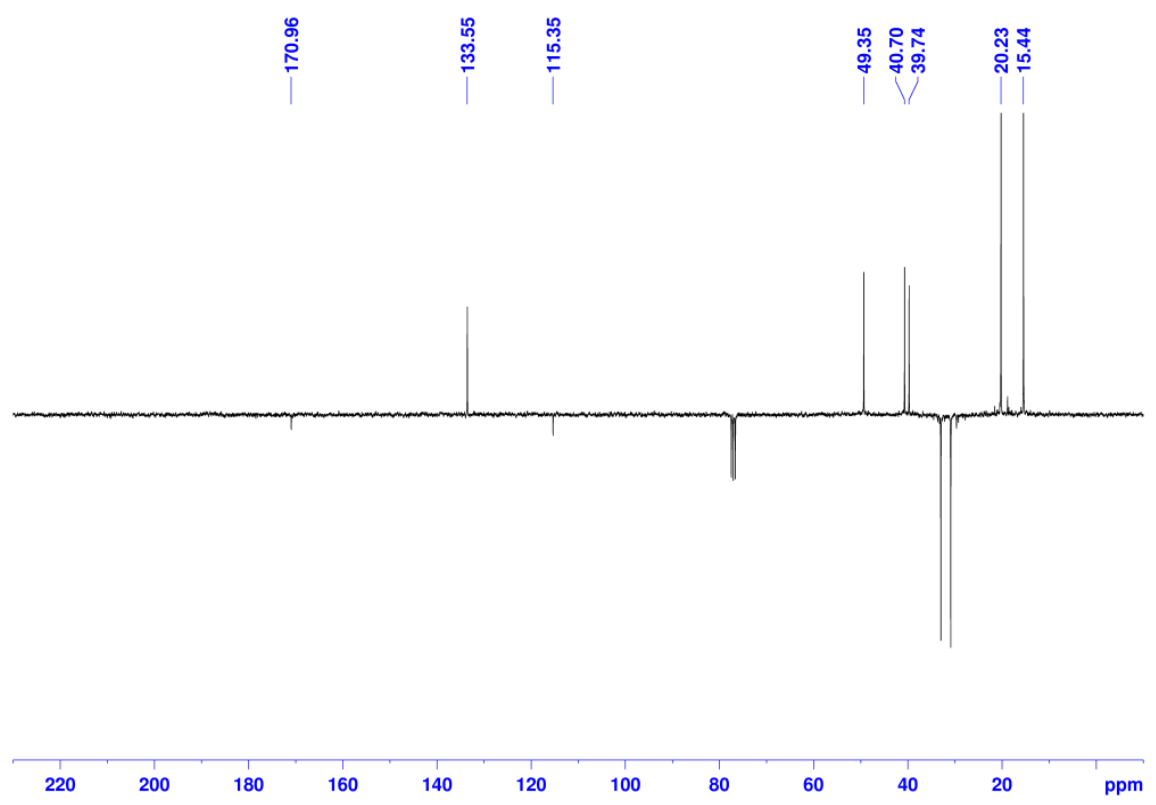

Figure $S 139{ }^{13} \mathrm{C}(150 \mathrm{MHz})$ DEPTQ NMR spectrum of rac-35 $\left(\mathrm{CDCl}_{3}, 298 \mathrm{~K}\right)$. 


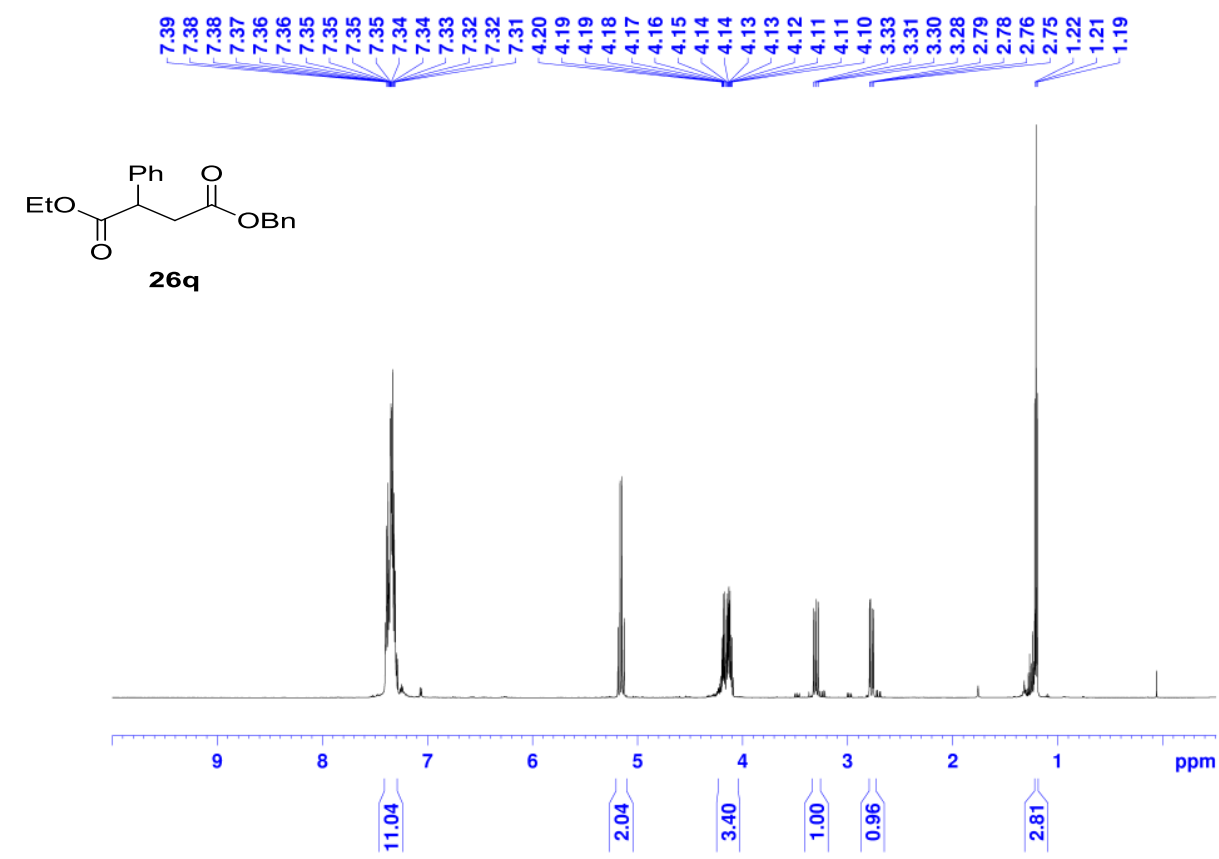

Figure $S 140{ }^{1} \mathrm{H}(600 \mathrm{MHz}) \mathrm{NMR}$ spectrum of $\mathbf{2 6 q}\left(\mathrm{CDCl}_{3}, 298 \mathrm{~K}\right)$.

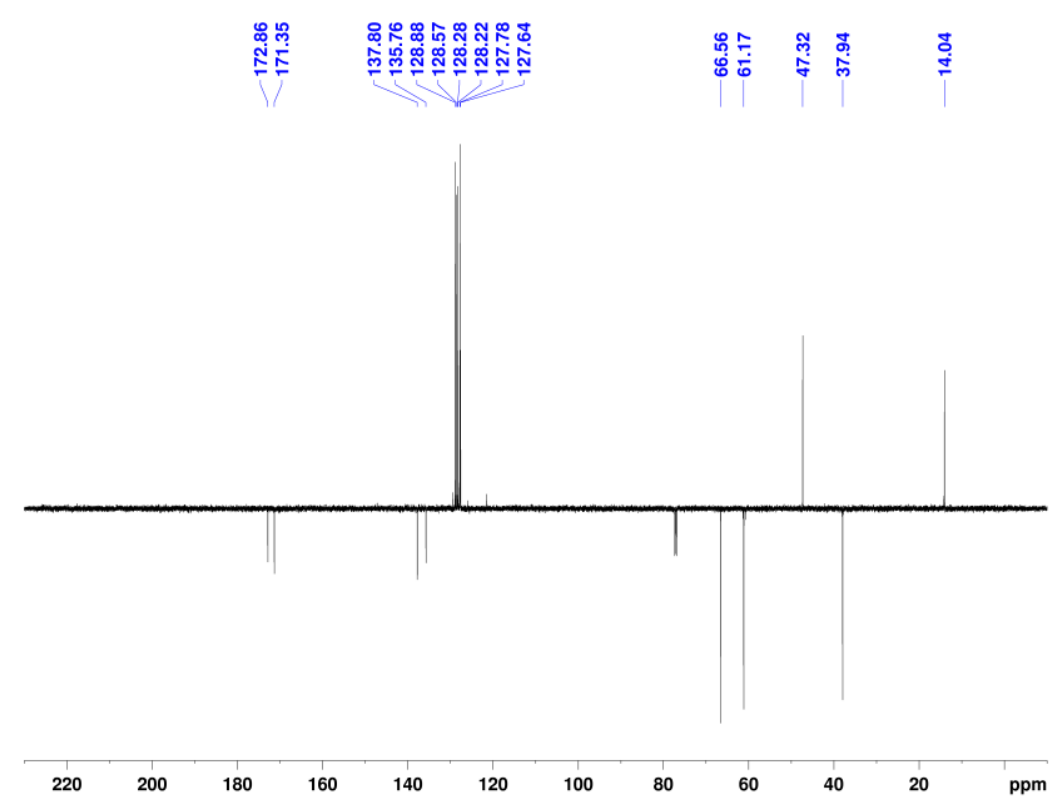

Figure $\mathbf{S 1 4 1}{ }^{13} \mathrm{C}(150 \mathrm{MHz})$ DEPTQ NMR spectrum of $\mathbf{2 6 q}\left(\mathrm{CDCl}_{3}, 298 \mathrm{~K}\right)$. 


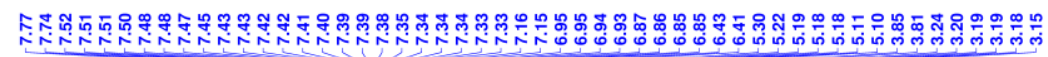
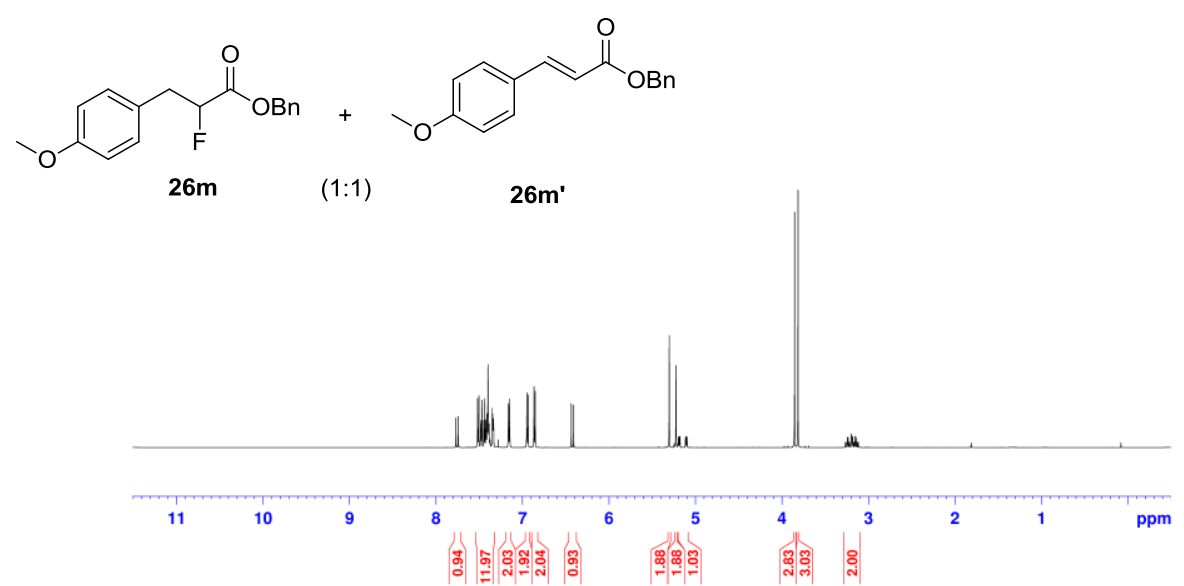

Figure $\mathbf{S 1 4 2}{ }^{1} \mathrm{H}(600 \mathrm{MHz}) \mathrm{NMR}$ spectrum of $\mathbf{2 6 m + 2 6 m}\left(\mathrm{CDCl}_{3}, 298 \mathrm{~K}\right)$.

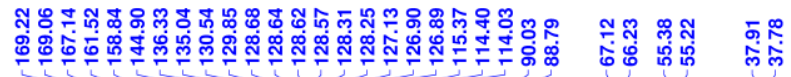

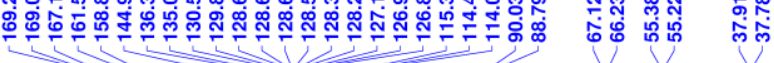

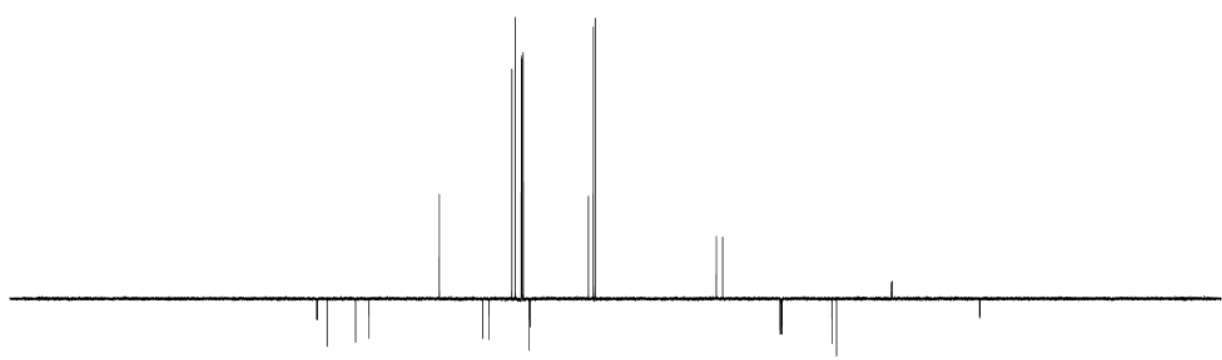

$\begin{array}{lllllllllllllllllllllll}220 & 210 & 200 & 190 & 180 & 170 & 160 & 150 & 140 & 130 & 120 & 110 & 100 & 90 & 80 & 70 & 60 & 50 & 40 & 30 & 20 & 10 & \mathrm{ppm}\end{array}$

Figure $\mathbf{S} 1433^{13} \mathrm{C}(150 \mathrm{MHz})$ DEPTQ NMR spectrum of $\mathbf{2 6 m + 2 6 m '}\left(\mathrm{CDCl}_{3}, 298 \mathrm{~K}\right)$. 


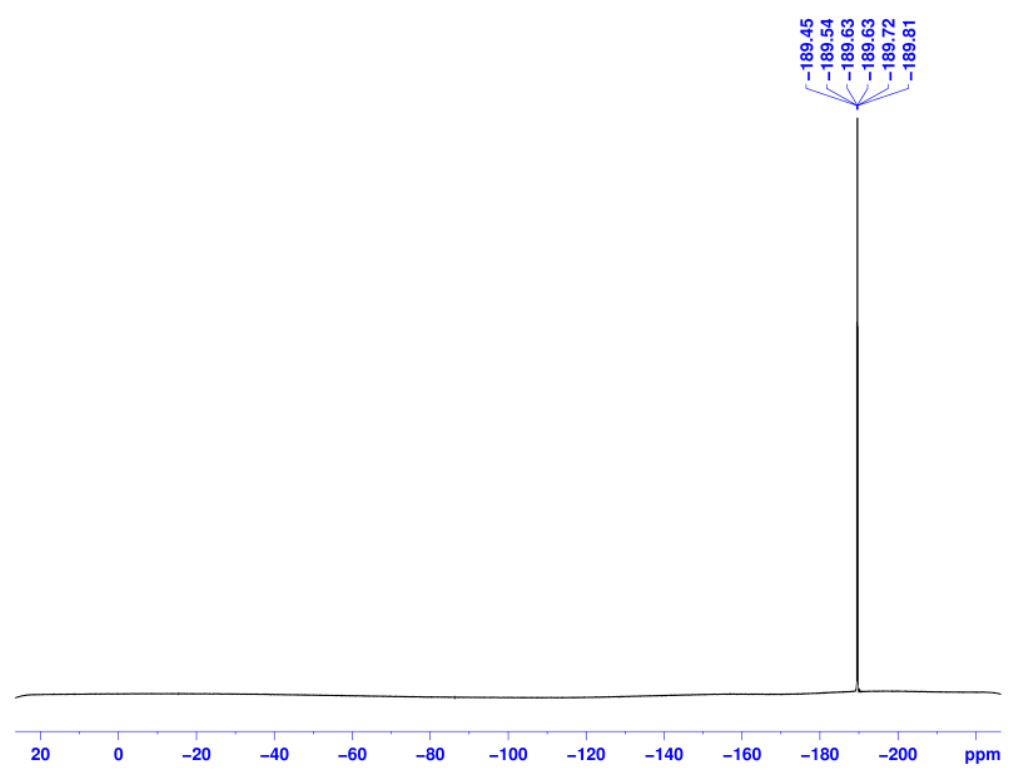

Figure $\mathbf{S 1 4 4}{ }^{19} \mathrm{~F}(280 \mathrm{MHz}) \mathrm{NMR}$ spectrum of $\mathbf{2 6 m + 2 6 m '}\left(\mathrm{CDCl}_{3}, 298 \mathrm{~K}\right)$. 


\section{X-ray data: Crystal data and structure refinement, selected geometric data, ORTEPs (Oak Ridge Thermal Ellipsoid Plot).}

\subsection{X-ray data of $3 b$}

Table 1. Crystal data and structure refinement for yvr2053-vor (CCDC: 1422415).

Identification code
Empirical formula
Moiety formula
Formula weight
Temperature
Wavelength
Crystal system
Space group

Unit cell dimensions

Volume

Z

Density (calculated)

Absorption coefficient

$\mathrm{F}(000)$

Crystal size

Theta range for data collection

Index ranges

Reflections collected

Independent reflections

Completeness to theta $=25.000^{\circ}$

Absorption correction

Refinement method

Data / restraints / parameters

Goodness-of-fit on $\mathrm{F}^{2}$

Final $R$ indices [l>2sigma(I)]

$\mathrm{R}$ indices (all data)

Extinction coefficient

Largest diff. peak and hole yvr2053-vor

C32 H46 N2 O

$\mathrm{C} 32 \mathrm{H} 46 \mathrm{~N} 2 \mathrm{O}$

474.71

100(2) K

$0.71073 \AA$

Monoclinic

$\mathrm{C} 2 / \mathrm{c}$

$\mathrm{a}=39.090(8) \AA$

$\alpha=90^{\circ}$.

$\mathrm{b}=9.747(2) \AA$

$\beta=116.156(7)^{\circ}$.

$\mathrm{c}=17.279(4) \AA$

$\gamma=90^{\circ}$.
8

$1.067 \mathrm{Mg} / \mathrm{m}^{3}$

$0.063 \mathrm{~mm}^{-1}$

2080

$0.150 \times 0.150 \times 0.030 \mathrm{~mm}^{3}$

1.161 to $25.000^{\circ}$.

$-46<=\mathrm{h}<=46,-1<=\mathrm{k}<=11,-20<=\mathrm{l}<=20$

13767

$5068[R($ int $)=0.1051]$

$97.5 \%$

None

Full-matrix least-squares on $\mathrm{F}^{2}$

5068 / 0 / 325

1.009

$\mathrm{R} 1=0.0982, \mathrm{wR} 2=0.2393$

$R 1=0.2547, w R 2=0.3044$

$\mathrm{n} / \mathrm{a}$

0.360 and -0.212 e. $\AA^{-3}$ 


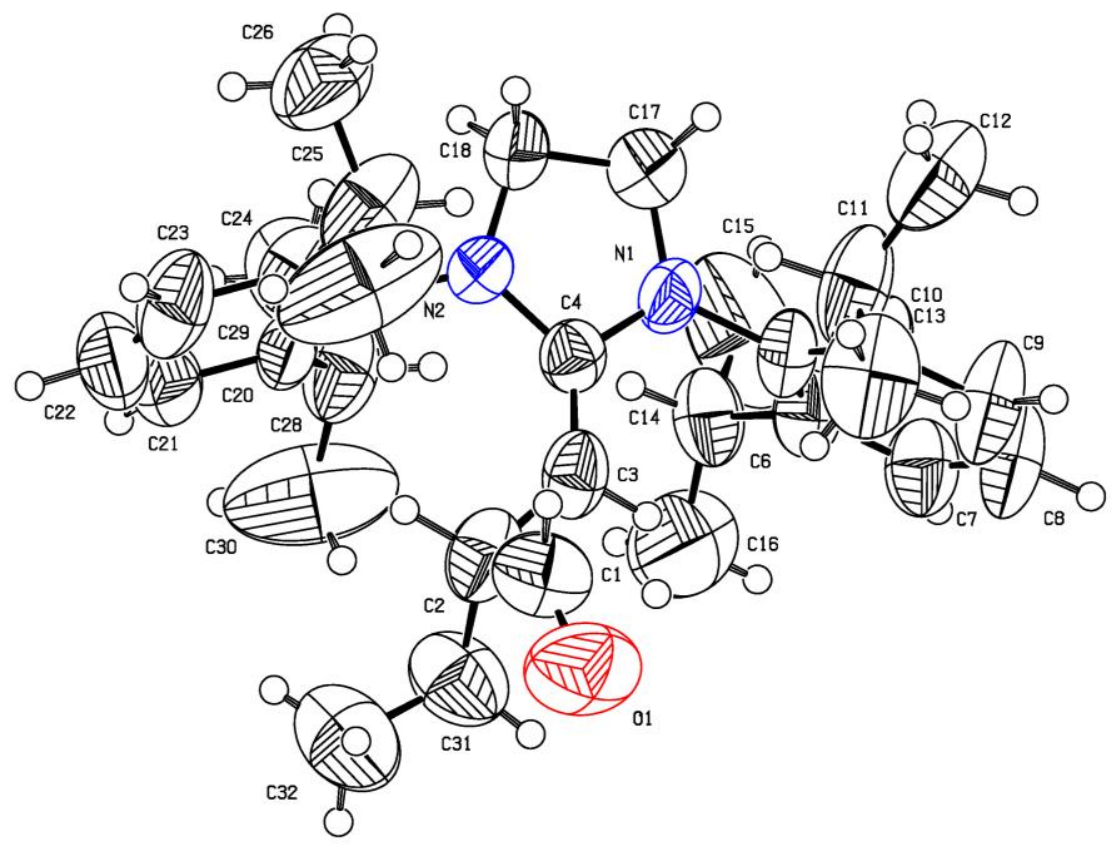

Figure S145. ORTEP of the X-ray crystal structure of $\mathbf{3 b}$. Thermal ellipsoids are drawn at $50 \%$ probability level.

\subsection{X-ray data of 10}

Table 1. Crystal data and structure refinement for yvr3125 (CCDC: 1422406).

Identification code

Empirical formula

Moiety formula

Formula weight

Temperature

Wavelength

Crystal system

Space group

Unit cell dimensions

Volume

Z

Density (calculated)

Absorption coefficient yvr3125

C38 H50 N2 O

C38 H50 N2 O

550.80

$100(2) \mathrm{K}$

$1.54178 \AA$

Monoclinic

$\mathrm{P} 2{ }_{1} / \mathrm{C}$

$a=14.8524(4) \AA$

$\alpha=90^{\circ}$.

$\mathrm{b}=11.9256(3) \AA$

$\beta=108.7930(10)^{\circ}$.

$c=19.3793(5) \AA$

3249.54(15) $\AA^{3}$

4

$1.126 \mathrm{Mg} / \mathrm{m}^{3}$

$0.505 \mathrm{~mm}^{-1}$ 
$\mathrm{F}(000)$

Crystal size

Theta range for data collection

Index ranges

Reflections collected

Independent reflections

Completeness to theta $=67.679^{\circ}$

Absorption correction

Max. and min. transmission

Refinement method

Data / restraints / parameters

Goodness-of-fit on F2

Final $R$ indices [ $>2$ sigma(I)]

$R$ indices (all data)

Extinction coefficient

Largest diff. peak and hole
1200

$0.300 \times 0.200 \times 0.100 \mathrm{~mm}^{3}$

4.422 to $72.177^{\circ}$.

$-14<=\mathrm{h}<=18,-14<=\mathrm{k}<=14,-23<=\mathrm{l}<=23$

36505

$6225[R($ int $)=0.0409]$

$97.5 \%$

Multiscan

0.7536 and 0.6109

Full-matrix least-squares on $\mathrm{F}^{2}$

6225 / 0 / 387

0.959

$\mathrm{R} 1=0.0439, \mathrm{wR} 2=0.1137$

$\mathrm{R} 1=0.0474, \mathrm{wR} 2=0.1221$

$\mathrm{n} / \mathrm{a}$

0.284 and -0.304 e. $\AA^{-3}$

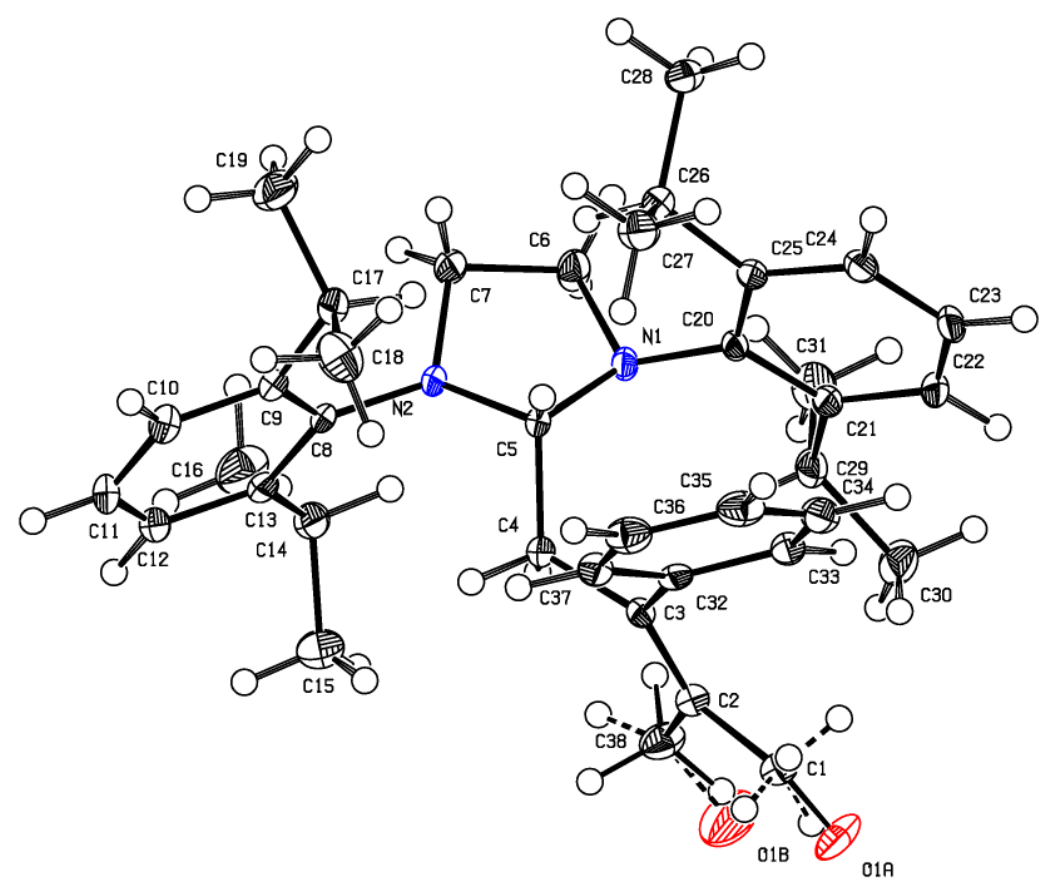

Figure S146. ORTEP of the X-ray crystal structure of 10 with disordered formyl/methyl groups (E/Z). Thermal ellipsoids are drawn at $50 \%$ probability level. 


\subsection{X-ray data of $15 \mathrm{a} \cdot \mathrm{HCl}$}

Table 1. Crystal data and structure refinement for yvr2124(CCDC:1422412).

Identification code

Empirical formula

Moiety formula

Formula weight

Temperature

Wavelength

Crystal system

Space group

Unit cell dimensions

Volume

Z

Density (calculated)

Absorption coefficient

$\mathrm{F}(000)$

Crystal size

Theta range for data collection

Index ranges

Reflections collected

Independent reflections

Completeness to theta $=25.242^{\circ}$

Absorption correction

Refinement method

Data / restraints / parameters

Goodness-of-fit on F2

Final $R$ indices [l>2sigma(I)]

$\mathrm{R}$ indices (all data)

Extinction coefficient

Largest diff. peak and hole yvr2124

$\mathrm{C} 23 \mathrm{H} 22 \mathrm{Cl}$ N3

$\mathrm{C} 23 \mathrm{H} 22 \mathrm{~N} 3, \mathrm{Cl}$

375.88

100(2) K

$0.71073 \AA$

Triclinic

P-1

$a=9.4535(3) \AA$ $\alpha=68.473(2)^{\circ}$.

$\mathrm{b}=9.8004(4) \AA$

$\beta=80.394(2)^{\circ}$.

$c=11.8659(4) \AA$

$\gamma=78.845(2)^{\circ}$.
2

$1.251 \mathrm{Mg} / \mathrm{m}^{3}$

$0.203 \mathrm{~mm}^{-1}$

396

$0.400 \times 0.300 \times 0.300 \mathrm{~mm}^{3}$

1.855 to $26.999^{\circ}$.

$-12<=\mathrm{h}<=8,-12<=\mathrm{k}<=12,-14<=\mathrm{k}<=15$

6379

$4346[R($ int $)=0.0163]$

$99.9 \%$

None

Full-matrix least-squares on $\mathrm{F}^{2}$

4346 / 0 / 247

1.053

$\mathrm{R} 1=0.0372, \mathrm{wR} 2=0.0964$

$\mathrm{R} 1=0.0479, \mathrm{wR} 2=0.1016$

$\mathrm{n} / \mathrm{a}$

0.758 and -0.325 e. $\AA^{-3}$ 


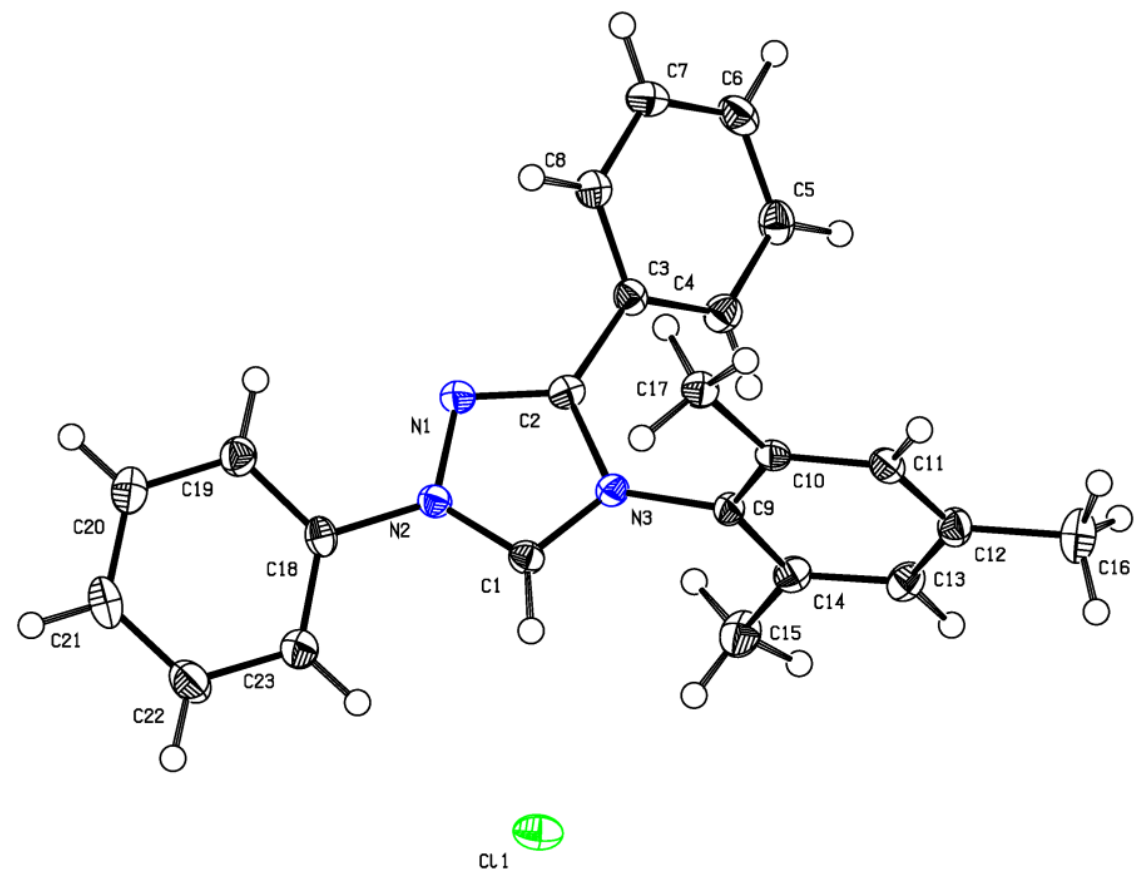

Figure S147. ORTEP of the X-ray crystal structure of $15 a \cdot \mathrm{HCl}$. Thermal ellipsoids are drawn at $50 \%$ probability level.

\section{$12.4 \mathrm{X}$-ray data of $15 \mathrm{~b} \cdot \mathrm{HCl}$}

Table 1. Crystal data and structure refinement for yvr2122 (CCDC:1422413).

Identification code

Empirical formula

Moiety formula

Formula weight

Temperature

Wavelength

Crystal system

Space group

Unit cell dimensions

Volume

Z

Density (calculated)

Absorption coefficient yvr2122

$\mathrm{C} 26 \mathrm{H} 28 \mathrm{Cl} \mathrm{N} 3$

$\mathrm{C} 26 \mathrm{H} 28 \mathrm{~N} 3, \mathrm{Cl}$

417.96

100(2) K

$0.71073 \AA$

Monoclinic

$\mathrm{Cc}$

$a=19.5238(7) \AA \quad \alpha=90^{\circ}$.

$\mathrm{b}=13.2578(6) \AA \quad \beta=110.329(2)^{\circ}$.

C $=9.2969(3) \AA \quad \gamma=90^{\circ}$.

2256.54(15) $\AA^{3}$

4

$1.230 \mathrm{Mg} / \mathrm{m}^{3}$

$0.187 \mathrm{~mm}^{-1}$ 
$\mathrm{F}(000)$

Crystal size

Theta range for data collection

Index ranges

Reflections collected

Independent reflections

Completeness to theta $=25.242^{\circ}$

Absorption correction

Refinement method

Data / restraints / parameters

Goodness-of-fit on $\mathrm{F}^{2}$

Final $R$ indices [l>2sigma(I)]

$R$ indices (all data)

Absolute structure parameter

Extinction coefficient

Largest diff. peak and hole
888

$0.300 \times 0.200 \times 0.100 \mathrm{~mm}^{3}$

1.897 to $26.999^{\circ}$.

$-21<=\mathrm{h}<=24,-15<=\mathrm{k}<=16,-11<=\mathrm{k}<=10$

5541

$3545[R($ int $)=0.0241]$

$99.8 \%$

None

Full-matrix least-squares on $\mathrm{F}^{2}$

3545 / 2 / 275

1.035

$\mathrm{R} 1=0.0272, \mathrm{wR} 2=0.0655$

$\mathrm{R} 1=0.0294, \mathrm{wR} 2=0.0664$

$-0.01(3)$

$\mathrm{n} / \mathrm{a}$

0.157 and -0.160 e. $\AA^{-3}$

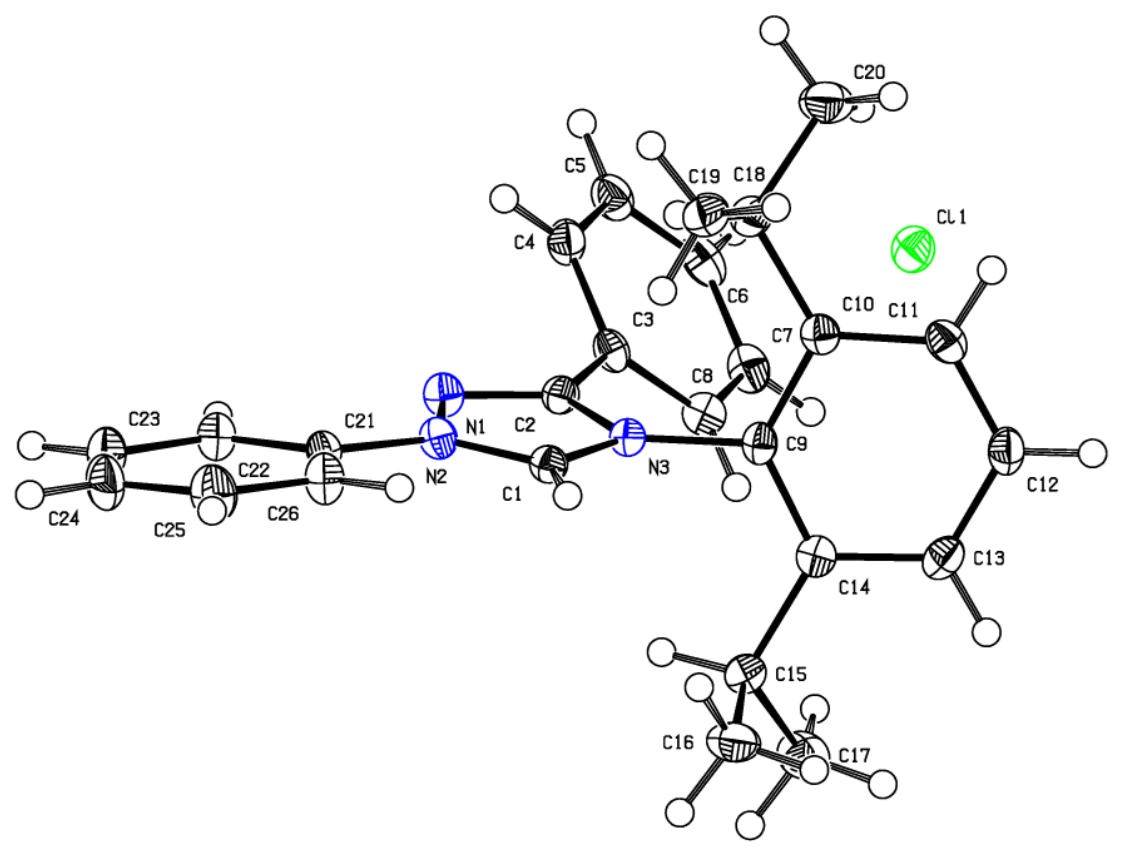

Figure S148. ORTEP of the X-ray crystal structure of $\mathbf{1 5 b} \cdot \mathrm{HCl}$ Thermal ellipsoids are drawn at $50 \%$ probability level. 


\subsection{X-ray data of 19}

Table 1. Crystal data and structure refinement for yvr2151n (CCDC:1422410).

Identification code

Empirical formula

Moiety formula

Formula weight

Temperature

Wavelength

Crystal system

Space group

Unit cell dimensions

Volume

Z

Density (calculated)

Absorption coefficient

$\mathrm{F}(000)$

Crystal size

Theta range for data collection

Index ranges

Reflections collected

Independent reflections

Completeness to theta $=67.684^{\circ}$

Absorption correction

Refinement method

Data / restraints / parameters

Goodness-of-fit on $\mathrm{F}^{2}$

Final $R$ indices [l>2sigma( $(\mathrm{l})]$

$\mathrm{R}$ indices (all data)

Extinction coefficient

Largest diff. peak and hole yvr2151n

$\mathrm{C} 41 \mathrm{H} 44 \mathrm{~N} 4$

$\mathrm{C} 41 \mathrm{H} 44 \mathrm{~N} 4$

592.80

$100(2) \mathrm{K}$

$1.54184 \AA$

Monoclinic

$\mathrm{P} 2 / \mathrm{c}$

$a=12.1055(3) \AA \quad \alpha=90^{\circ}$.

$\mathrm{b}=11.3610(3) \AA \quad \beta=92.179(2)^{\circ}$.

$c=24.5577(5) \AA \quad \gamma=90^{\circ}$.

$3374.99(14) \AA^{3}$

4

$1.167 \mathrm{Mg} / \mathrm{m}^{3}$

$0.522 \mathrm{~mm}^{-1}$

1272

$0.100 \times 0.100 \times 0.030 \mathrm{~mm}^{3}$

3.654 to $65.018^{\circ}$.

$-14<=\mathrm{h}<=13,-12<=\mathrm{k}<=12,-28<=\mid<=11$

13409

$5260[R$ (int) $=0.0361]$

$86.1 \%$

None

Full-matrix least-squares on $\mathrm{F}^{2}$

5260 / 0 / 419

1.039

$\mathrm{R} 1=0.0457, \mathrm{wR} 2=0.1128$

$\mathrm{R} 1=0.0561, \mathrm{wR} 2=0.1197$

$\mathrm{n} / \mathrm{a}$

0.171 and -0.233 e. $\AA^{-3}$ 


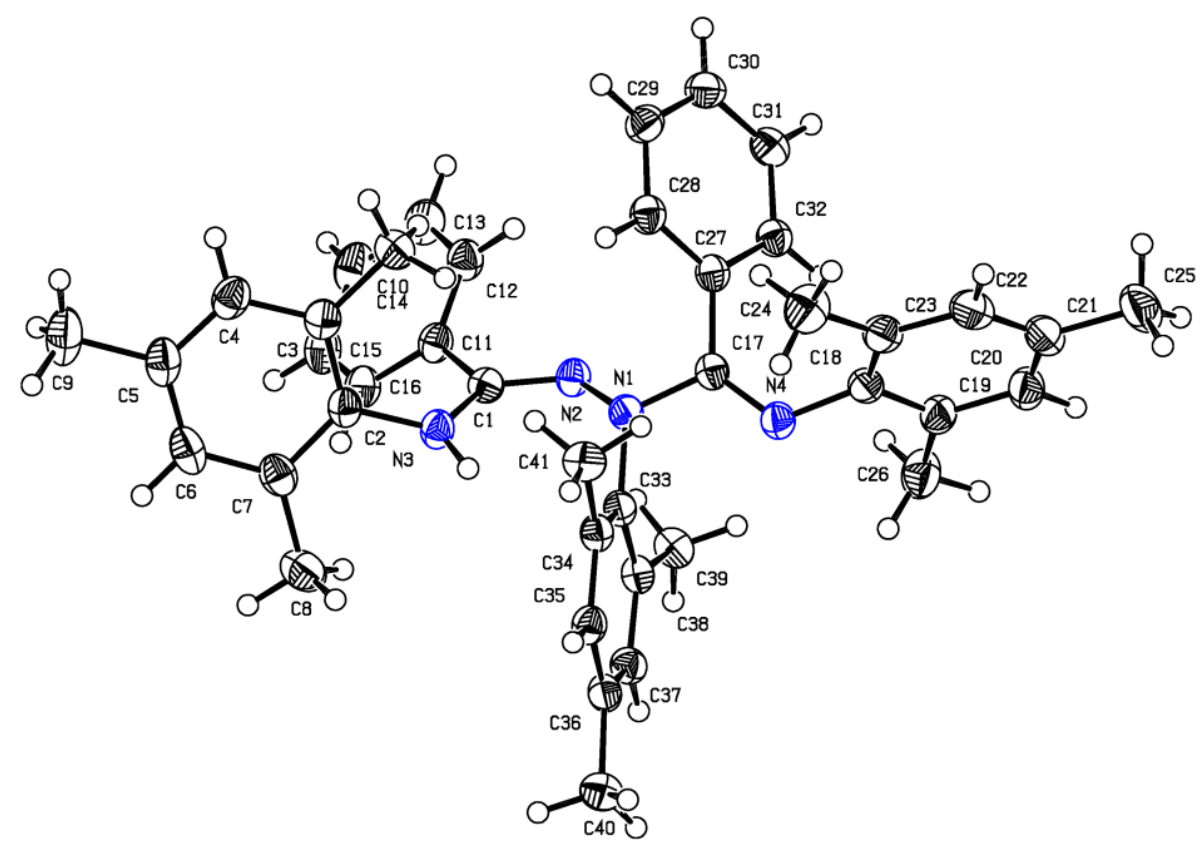

Figure S149. ORTEP of the X-ray crystal structure of 19. Thermal ellipsoids are drawn at $50 \%$ probability level.

\subsection{X-ray data of $21 \mathrm{a} \cdot \mathrm{HCl}$}

Table 1. Crystal data and structure refinement for yvr3001n (CCDC:1422408). Identification code

Empirical formula

Moiety formula

Formula weight

Temperature

Wavelength

Crystal system

Space group

Unit cell dimensions

Volume

Z

Density (calculated) yvr3001n

$\mathrm{C} 26 \mathrm{H} 28 \mathrm{Cl} \mathrm{N} 3$

C26 H28 N3, Cl

417.96

100(2) K

$0.71073 \AA$

Triclinic

P-1

$a=11.3897(17) \AA \quad \alpha=88.843(11)^{\circ}$.

$\mathrm{b}=12.4568(19) \AA \quad \beta=78.415(9)^{\circ}$.

$\mathrm{c}=18.339(3) \AA \quad \gamma=64.417(8)^{\circ}$.
4

$1.211 \mathrm{Mg} / \mathrm{m}^{3}$ 
Absorption coefficient

$\mathrm{F}(000)$

Crystal size

Theta range for data collection

Index ranges

Reflections collected

Independent reflections

Completeness to theta $=25.242^{\circ}$

Absorption correction

Refinement method

Data / restraints / parameters

Goodness-of-fit on $\mathrm{F}^{2}$

Final $\mathrm{R}$ indices [ $>2$ sigma(I)]

$\mathrm{R}$ indices (all data)

Extinction coefficient

Largest diff. peak and hole
$0.184 \mathrm{~mm}^{-1}$

888

$0.200 \times 0.070 \times 0.030 \mathrm{~mm}^{3}$

1.137 to $27.000^{\circ}$.

$-14<=\mathrm{h}<=14,-15<=\mathrm{k}<=15,-23<=\mathrm{k}<=23$

13660

$9407[R($ int $)=0.1231]$

$95.7 \%$

None

Full-matrix least-squares on $\mathrm{F}^{2}$

9407 / 0 / 553

0.900

$\mathrm{R} 1=0.0884, \mathrm{wR} 2=0.1852$

$\mathrm{R} 1=0.3024, \mathrm{wR} 2=0.2570$

$\mathrm{n} / \mathrm{a}$

0.644 and -0.308 e. $\AA^{-3}$

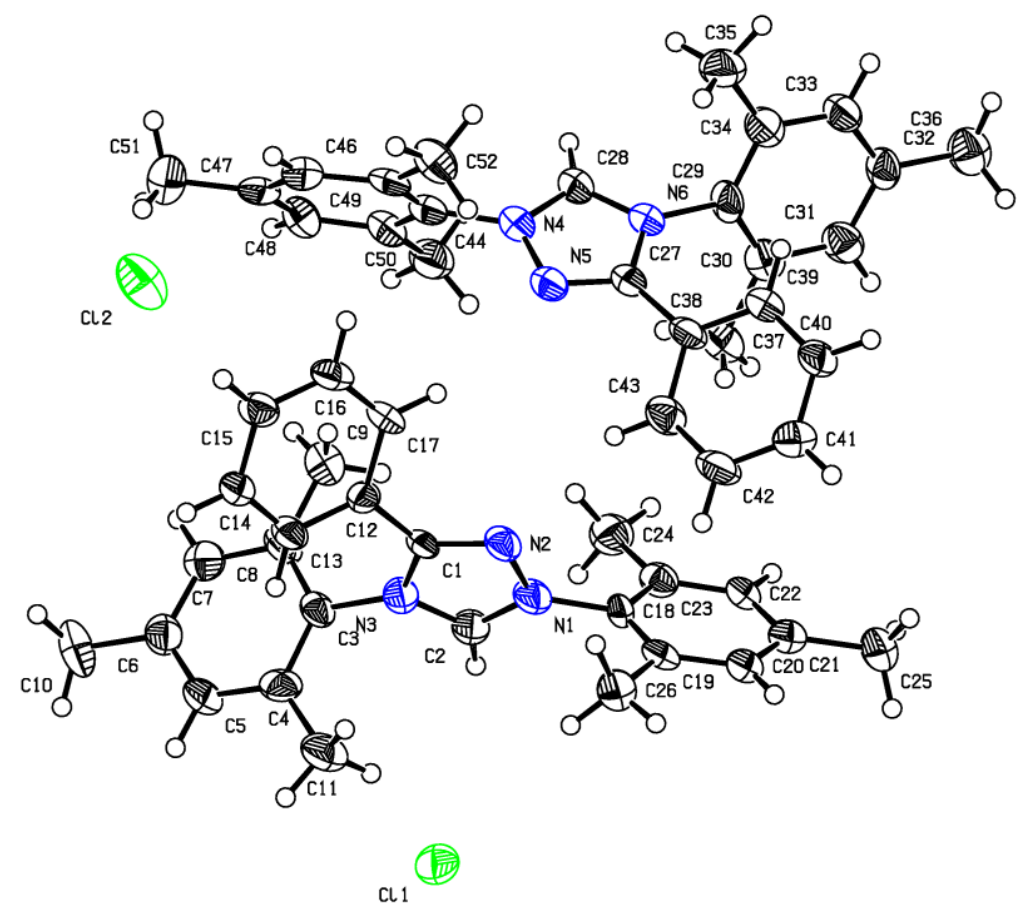

Figure S150. ORTEP of the X-ray crystal structure of $21 a \cdot H C l$ (two slightly different independent molecules per unit cell). Thermal ellipsoids are drawn at $50 \%$ probability level. 


\subsection{X-ray data of $21 \mathrm{~b} \cdot \mathrm{HCl}$}

Table 1. Crystal data and structure refinement for yvr2160-vor (CCDC:1422409).

Identification code

Empirical formula

Moiety formula

Formula weight

Temperature

Wavelength

Crystal system

Space group

Unit cell dimensions

Volume

Z

Density (calculated)

Absorption coefficient

$\mathrm{F}(000)$

Crystal size

Theta range for data collection

Index ranges

Reflections collected

Independent reflections

Completeness to theta $=24.999^{\circ}$

Absorption correction

Refinement method

Data / restraints / parameters

Goodness-of-fit on F2

Final $R$ indices [l>2sigma(I)]

$R$ indices (all data)

Extinction coefficient

Largest diff. peak and hole yvr2160-vor

C65 H82 Cl4 N6

2(C32 H40 N3), C H2 Cl2, 2(Cl)

1089.16

100(2) K

$0.71073 \AA$

Triclinic

$\mathrm{P}-1$

$a=10.2045(8) \AA$ $\alpha=66.015(3)^{\circ}$.

$b=12.1028(7) \AA$

$\beta=88.887(4)^{\circ}$.

C $=14.5479(7) \AA$

$\gamma=69.982(3)^{\circ}$.
1

$1.184 \mathrm{Mg} / \mathrm{m}^{3}$

$0.238 \mathrm{~mm}^{-1}$

582

$0.300 \times 0.200 \times 0.070 \mathrm{~mm}^{3}$

1.547 to $24.999^{\circ}$.

$-12<=h<=11,-14<=k<=14,-17<=\mid<=17$

8367

$5381[R$ (int) $=0.0298]$

$99.9 \%$

None

Full-matrix least-squares on $\mathrm{F}^{2}$

$5381 / 0 / 357$

1.083

$\mathrm{R} 1=0.0578, \mathrm{wR} 2=0.1426$

$\mathrm{R} 1=0.1006, \mathrm{wR} 2=0.1622$

$\mathrm{n} / \mathrm{a}$

0.812 and -0.703 e. $\AA^{-3}$ 


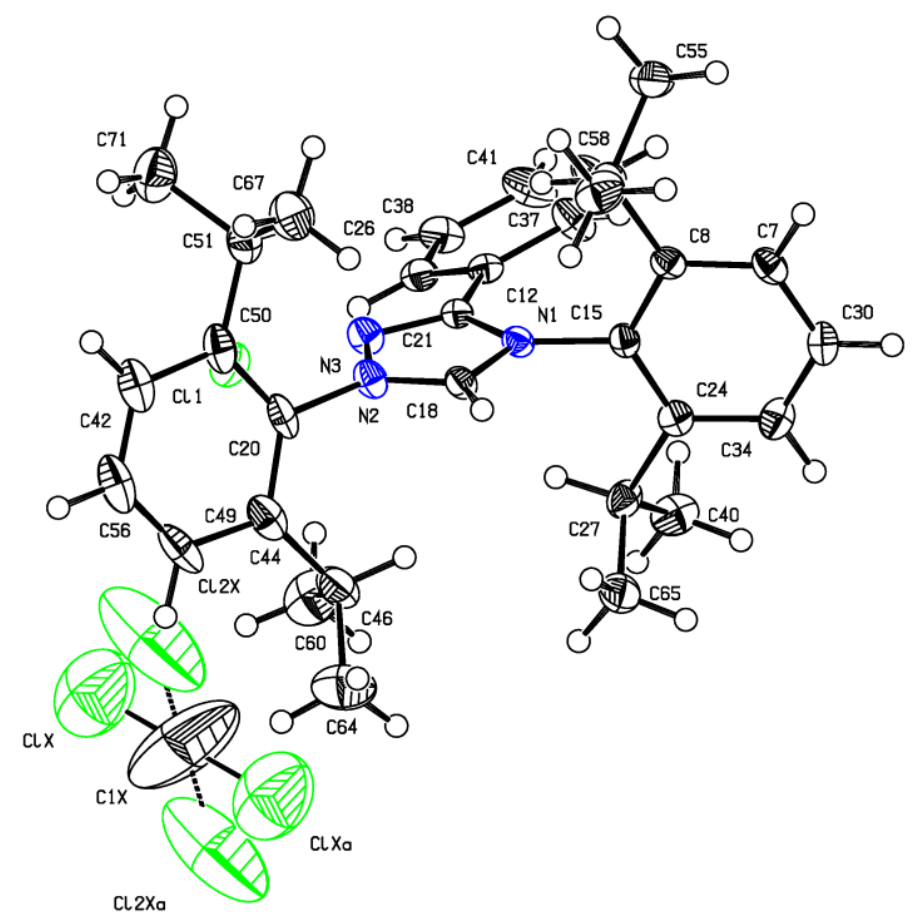

Figure S151. ORTEP of the X-ray crystal structure of $\mathbf{2 1} \mathbf{b} \cdot \mathrm{HCl}$ (containing disordered DCM molecule).Thermal ellipsoids are drawn at $50 \%$ probability level.

\subsection{X-ray data of $21 \mathrm{c} \cdot \mathrm{HBPh}_{4}$}

Table 1. Crystal data and structure refinement for dko54 (CCDC:1422417).

Identification code

Empirical formula

Moiety formula

Formula weight

Temperature

Wavelength

Crystal system

Space group

Unit cell dimensions

Volume

Z

Density (calculated)

Absorption coefficient dko54

C50 H48 B N3

C26 H28 N3, C24 H2O B

701.72

100(2) K

$0.71073 \AA$

Monoclinic

$\mathrm{P} 21 / \mathrm{c}$

$a=10.8366(2) \AA \quad \alpha=90^{\circ}$.

$\mathrm{b}=13.7520(3) \AA \quad \beta=100.1290(10)^{\circ}$.

c $=27.2542(5) \AA \quad \gamma=90^{\circ}$.

3998.25(14) ^3

4

$1.166 \mathrm{Mg} / \mathrm{m}^{3}$

$0.067 \mathrm{~mm}^{-1}$ 
$\mathrm{F}(000)$

Crystal size

Theta range for data collection

Index ranges

Reflections collected

Independent reflections

Completeness to theta $=27.00^{\circ}$

Absorption correction

Refinement method

Data / restraints / parameters

Goodness-of-fit on $\mathrm{F}^{2}$

Final $R$ indices [l>2sigma(I)]

$R$ indices (all data)

Largest diff. peak and hole
1496

$.3 \times .2 \times .2 \mathrm{~mm}^{3}$

1.52 to $27.00^{\circ}$.

$-13<=\mathrm{h}<=13,-17<=\mathrm{k}<=17,-34<=\mathrm{k}<=34$

33862

$8738[R$ (int) $=0.0511]$

$100.0 \%$

None

Full-matrix least-squares on $\mathrm{F}^{2}$

8738 / 0 / 491

1.000

$\mathrm{R} 1=0.0421, \mathrm{wR} 2=0.1076$

$\mathrm{R} 1=0.0731, \mathrm{wR} 2=0.1246$

0.373 and -0.277 e. $\AA^{-3}$
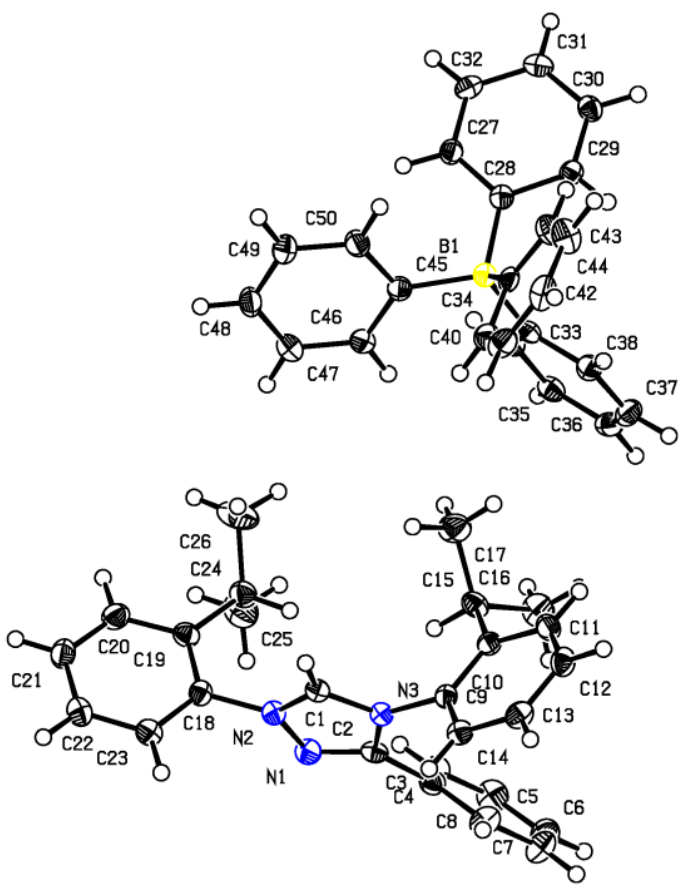

Figure S152. ORTEP of the X-ray crystal structure of $21 \mathrm{c} \cdot \mathrm{HBPh}_{4}$. Thermal ellipsoids are drawn at $50 \%$ probability level. 


\subsection{X-ray data of $21 \mathrm{~d} \bullet \mathrm{HBPh}_{4}$}

Table 1. Crystal data and structure refinement for dko60 (CCDC:1422416).

Identification code

Empirical formula

Moiety formula

Formula weight

Temperature

Wavelength

Crystal system

Space group

Unit cell dimensions

Volume

Z

Density (calculated)

Absorption coefficient

$\mathrm{F}(000)$

Crystal size

Theta range for data collection

Index ranges

Reflections collected

Independent reflections

Completeness to theta $=27.00^{\circ}$

Absorption correction

Refinement method

Data / restraints / parameters

Goodness-of-fit on $\mathrm{F}^{2}$

Final $R$ indices [l>2sigma( $(\mathrm{l})]$

$\mathrm{R}$ indices (all data)

Largest diff. peak and hole dko60

C56 H44 B N3

C32 H24 N3, C24 H2O B

769.75

100(2) K

$0.71073 \AA$

Monoclinic

p21/n

$a=20.9540(5) \AA \quad \alpha=90^{\circ}$.

$\mathrm{b}=14.0107(4) \AA \quad \beta=97.7840(10)^{\circ}$.

$c=28.6559(7) \AA \quad \gamma=90^{\circ}$.

8335.3(4) $\AA^{3}$

8

$1.227 \mathrm{Mg} / \mathrm{m}^{3}$

$0.071 \mathrm{~mm}^{-1}$

3248

$.3 \times .1 \times .03 \mathrm{~mm}^{3}$

1.13 to $27.00^{\circ}$.

$-26<=\mathrm{h}<=25,-17<=\mathrm{k}<=17,-31<=\mid<=36$

45642

$18191[R$ (int) $=0.0894]$

$100.0 \%$

None

Full-matrix least-squares on $\mathrm{F}^{2}$

18191 / 0 / 1081

0.883

$\mathrm{R} 1=0.0468, \mathrm{wR} 2=0.0882$

$\mathrm{R} 1=0.1378, \mathrm{wR} 2=0.1246$

0.240 and -0.236 e. $\AA^{-3}$ 


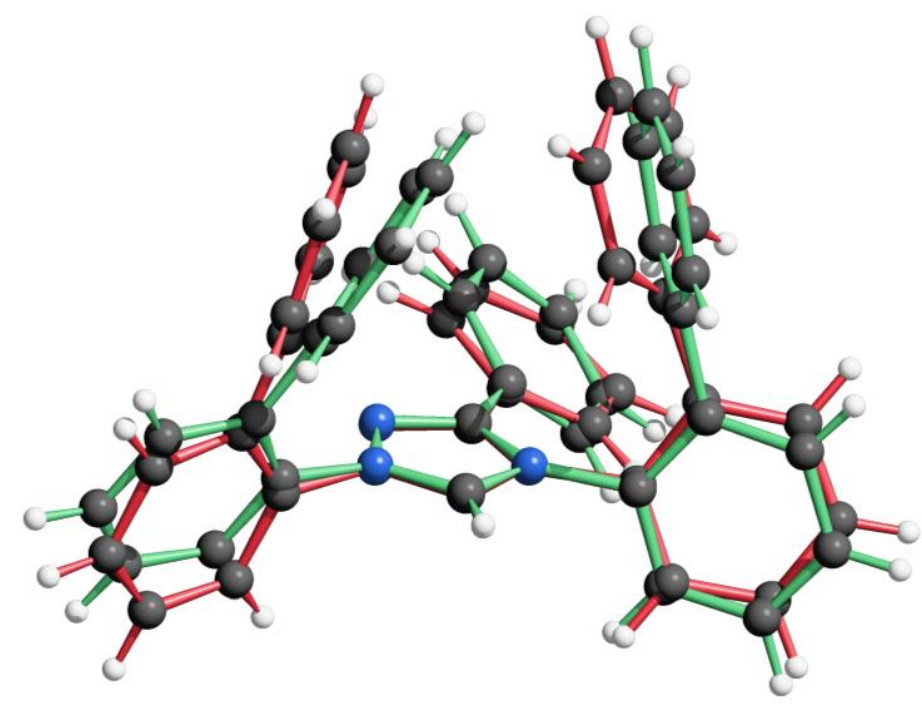

Figure S153. Overlayed structures of the independent molecules in the unit cell of 21d• $\mathrm{HBPh}_{4}$ (counter anions are omitted for clarity).

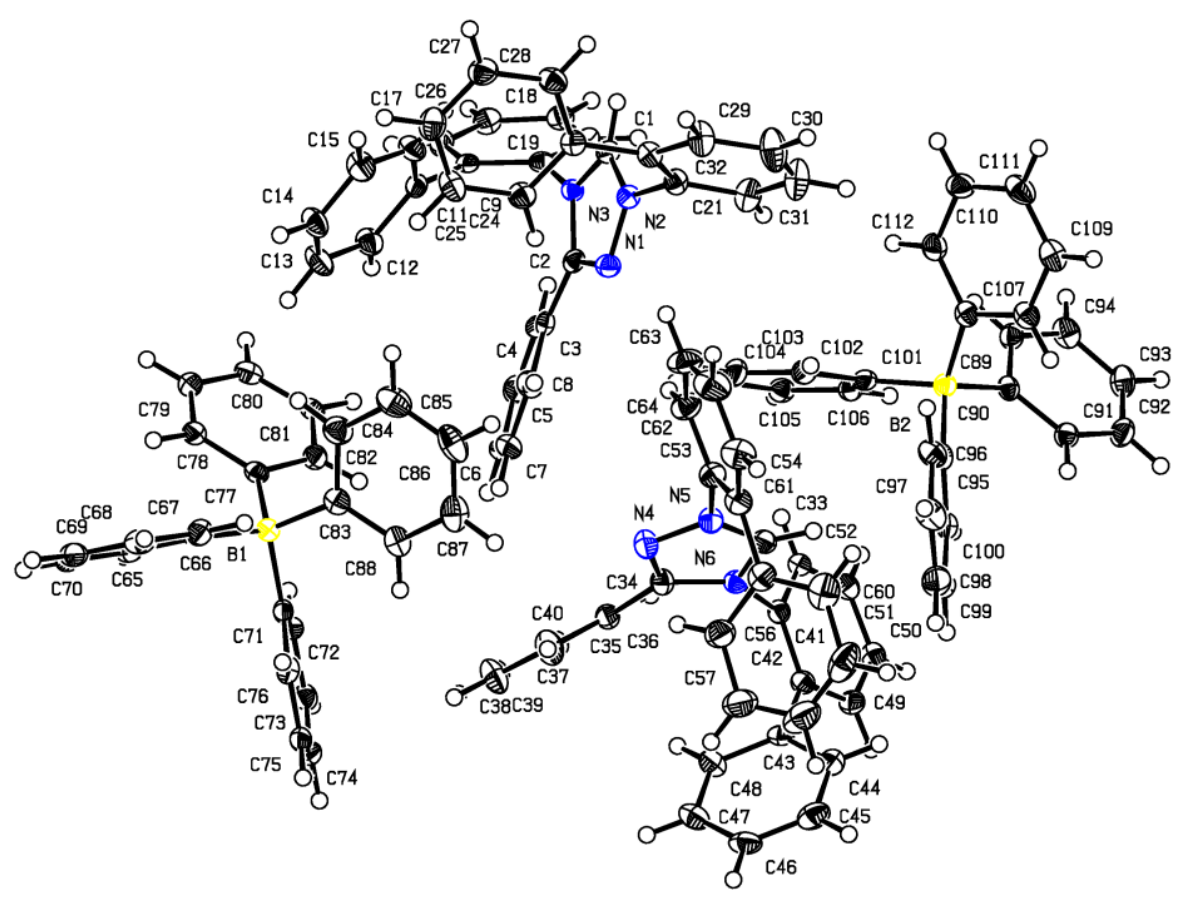

Figure S154. ORTEP of the X-ray crystal structure of $\mathbf{2 1 d} \cdot \mathrm{HBPh}_{4}$ (two molecules per unit cell). Thermal ellipsoids are drawn at $50 \%$ probability level. 


\subsection{X-ray data of 27}

Table 1. Crystal data and structure refinement for yvr2144 (CCDC:1422411).

Identification code

Empirical formula

Moiety formula

Formula weight

Temperature

Wavelength

Crystal system

Space group

Unit cell dimensions

Volume

Z

Density (calculated)

Absorption coefficient

$\mathrm{F}(000)$

Crystal size

Theta range for data collection

Index ranges

Reflections collected

Independent reflections

Completeness to theta $=25.242^{\circ}$

Absorption correction

Refinement method

Data / restraints / parameters

Goodness-of-fit on F2

Final $\mathrm{R}$ indices [l>2sigma(I)]

$R$ indices (all data)

Extinction coefficient

Largest diff. peak and hole yvr2144

C17 H26 Cl N3 O

C17 H24 N3, Cl, H2 O

323.86

100(2) K

$0.71073 \AA$

Orthorhombic

Pbca

$$
\begin{array}{ll}
\mathrm{a}=10.3313(4) \AA & \alpha=90^{\circ} . \\
\mathrm{b}=15.8693(6) \AA & \beta=90^{\circ} . \\
\mathrm{c}=21.6495(8) \AA & \gamma=90^{\circ} .
\end{array}
$$

3549.4(2) $\AA^{3}$

8

$1.212 \mathrm{Mg} / \mathrm{m}^{3}$

$0.221 \mathrm{~mm}^{-1}$

1392

$0.400 \times 0.400 \times 0.300 \mathrm{~mm}^{3}$

2.534 to $26.998^{\circ}$.

$-13<=\mathrm{h}<=12,-16<=\mathrm{k}<=20,-27<=\mathrm{|}<=26$

16390

$3871[R$ (int) $=0.0459]$

$99.9 \%$

None

Full-matrix least-squares on $\mathrm{F}^{2}$

3871 / 0 / 259

1.068

$\mathrm{R} 1=0.0366, \mathrm{wR} 2=0.0914$

$R 1=0.0585, w R 2=0.0980$

$\mathrm{n} / \mathrm{a}$

0.276 and -0.337 e. $\AA^{-3}$ 


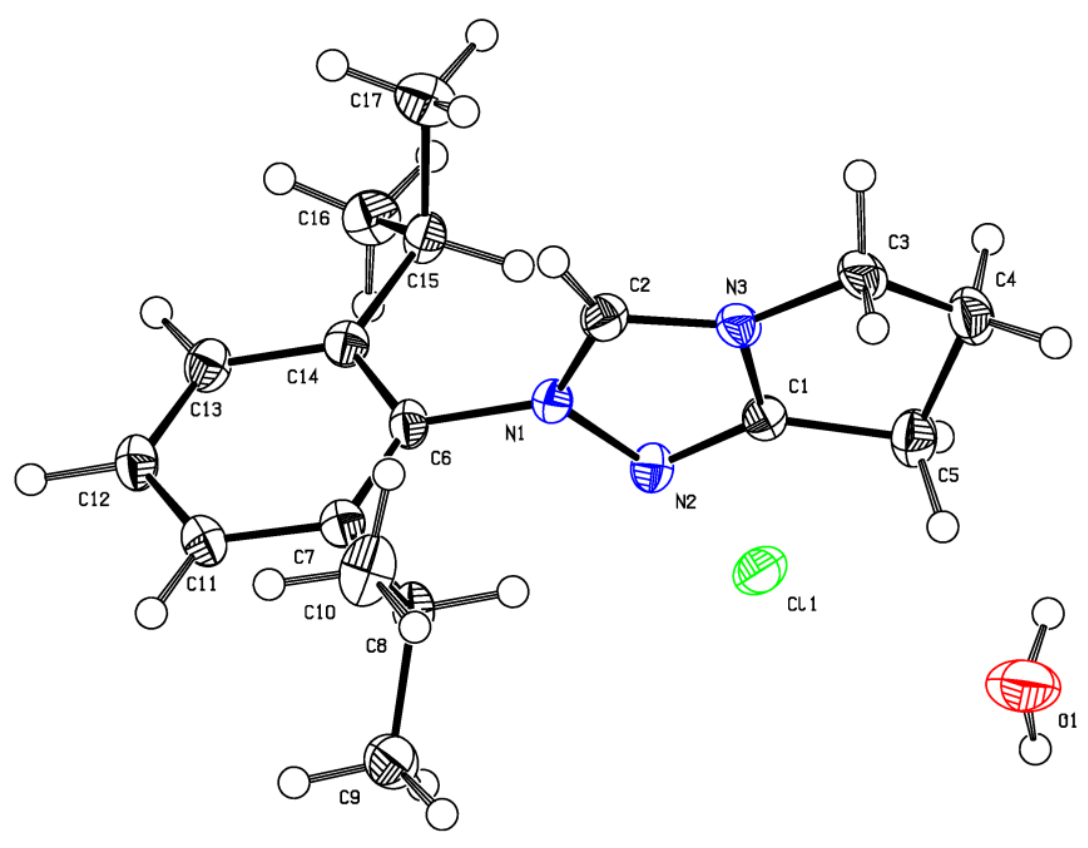

Figure S155. ORTEP of the X-ray crystal structure of 27 (containing 1 molecule of water). Thermal ellipsoids are drawn at $50 \%$ probability level.

\subsection{X-ray data of $21 b$}

Table 1. Crystal data and structure refinement for yvr3016-vor (CCDC:1422407). Identification code

Empirical formula

Moiety formula

Formula weight

Temperature

Wavelength

Crystal system

Space group

Unit cell dimensions

Volume

Z

Density (calculated)

Absorption coefficient yvr3016-vor

C32 H39 N3

C32 H39 N3

465.66

100(2) K

$0.71073 \AA$

Monoclinic

$\mathrm{P} 2{ }_{1} / \mathrm{C}$

$\mathrm{a}=24.0112(7) \AA$

$\alpha=90^{\circ}$.

$\mathrm{b}=10.1112(3) \AA$

$\beta=109.384(2)^{\circ}$.

$\mathrm{c}=24.1821(8) \AA$

$\gamma=90^{\circ}$.
8

$1.117 \mathrm{Mg} / \mathrm{m}^{3}$

$0.065 \mathrm{~mm}^{-1}$ 
$\mathrm{F}(000)$

Crystal size

Theta range for data collection

Index ranges

Reflections collected

Independent reflections

Completeness to theta $=25.242^{\circ}$

Absorption correction

Refinement method

Data / restraints / parameters

Goodness-of-fit on $\mathrm{F}^{2}$

Final $\mathrm{R}$ indices [l>2sigma(I)]

$\mathrm{R}$ indices (all data)

Extinction coefficient

Largest diff. peak and hole
2016

$0.200 \times 0.200 \times 0.150 \mathrm{~mm}^{3}$

0.899 to $26.999^{\circ}$.

$-30<=\mathrm{h}<=20,-12<=\mathrm{k}<=12,-27<=\mathrm{k}<=30$

22634

$11991[R($ int $)=0.0651]$

$99.2 \%$

None

Full-matrix least-squares on $\mathrm{F}^{2}$

11991 / 0 / 648

1.062

$\mathrm{R} 1=0.1149, \mathrm{wR} 2=0.3435$

$\mathrm{R} 1=0.1809, \mathrm{wR} 2=0.3677$

$0.0054(8)$

0.531 and -0.460 e. $\AA^{-3}$

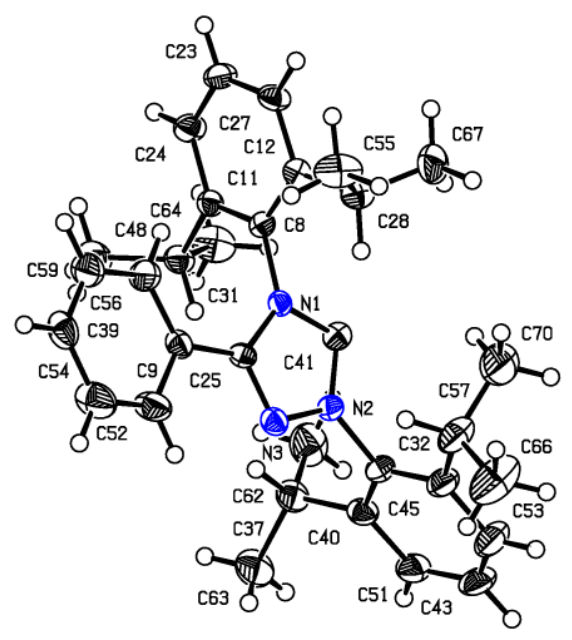

Figure S156. ORTEP of the X-ray crystal structure of 21b (two molecules per unit cell). Thermal ellipsoids are drawn at $50 \%$ probability level. 


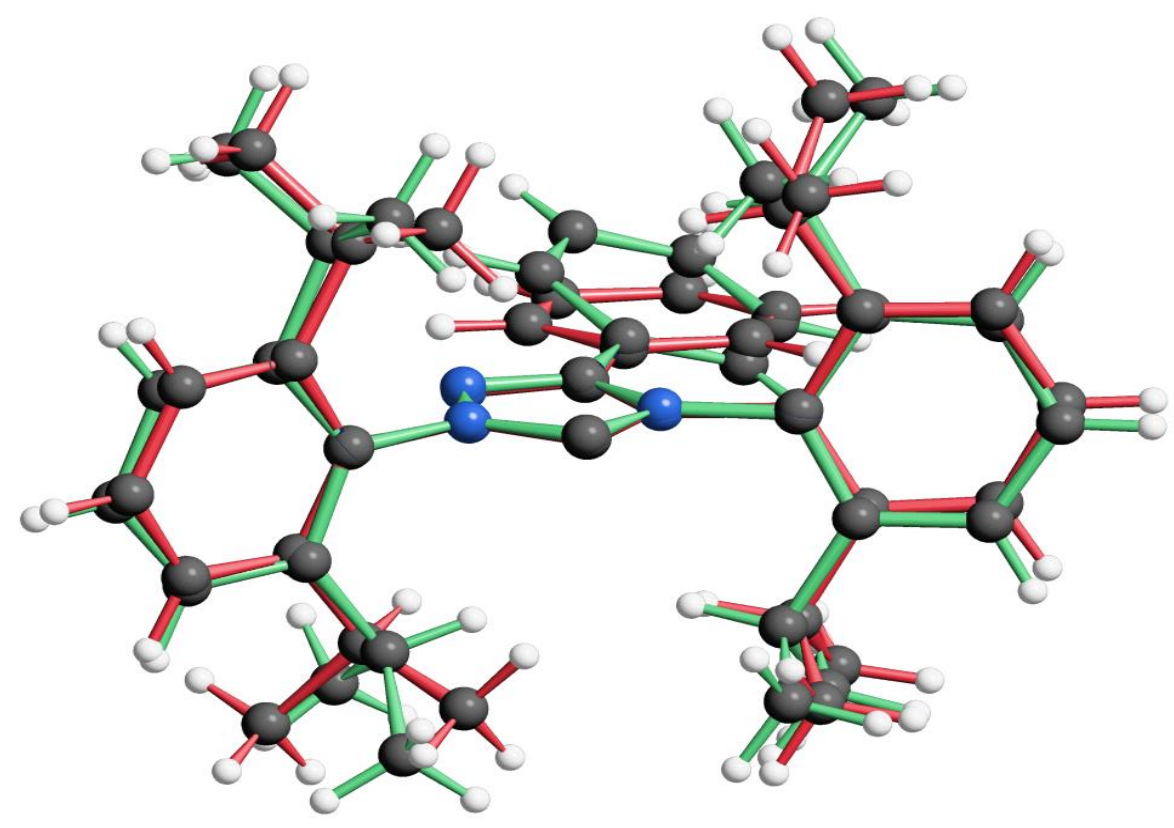

Figure S157. Overlayed structures of the independent molecules in the unit cell of 21b.

\subsection{X-ray data of $21 a$}

Table 1: Crystal data and structure refinement for yvr1092n (CCDC 1447520).

Identification code

Empirical formula

Moiety formula

Formula weight

Temperature

Wavelength

Crystal system

Space group

Unit cell dimensions

Volume

Z

Density (calculated)

Absorption coefficient yvr1092n

C26 H27 N3

$\mathrm{C} 26 \mathrm{H} 27 \mathrm{~N} 3$

381.50

100(2) K

$1.54178 \AA$

Monoclinic

$\mathrm{C} 2 / \mathrm{c}$

$a=31.8863(8) \AA$

$\alpha=90^{\circ}$.

$\mathrm{b}=8.0471(2) \AA$

$\beta=117.4740(10)^{\circ}$.

$c=19.3589(5) \AA$

4407.1(2) $\AA^{3}$

8

$1.150 \mathrm{Mg} / \mathrm{m}^{3}$

$0.522 \mathrm{~mm}^{-1}$ 
$\mathrm{F}(000)$

Crystal size

Theta range for data collection

Index ranges

Reflections collected

Independent reflections

Completeness to theta $=67.679^{\circ}$

Absorption correction

Max. and min. transmission

Refinement method

Data / restraints / parameters

Goodness-of-fit on F2

Final $\mathrm{R}$ indices [l>2sigma(I)]

$R$ indices (all data)

Extinction coefficient

Largest diff. peak and hole
1632

$0.250 \times 0.200 \times 0.030 \mathrm{~mm}^{3}$

3.124 to $72.150^{\circ}$.

$-39<=\mathrm{h}<=39,-9<=\mathrm{k}<=9,-23<=\mid<=23$

32389

$4330[R$ (int) $=0.0302]$

$99.8 \%$

Multiscan

0.7536 and 0.6609

Full-matrix least-squares on $\mathrm{F}^{2}$

4330 / 0 / 268

1.035

$\mathrm{R} 1=0.0384, \mathrm{wR} 2=0.1015$

$\mathrm{R} 1=0.0407, \mathrm{wR} 2=0.1036$

$\mathrm{n} / \mathrm{a}$

0.265 and -0.205 e. $\AA^{-3}$

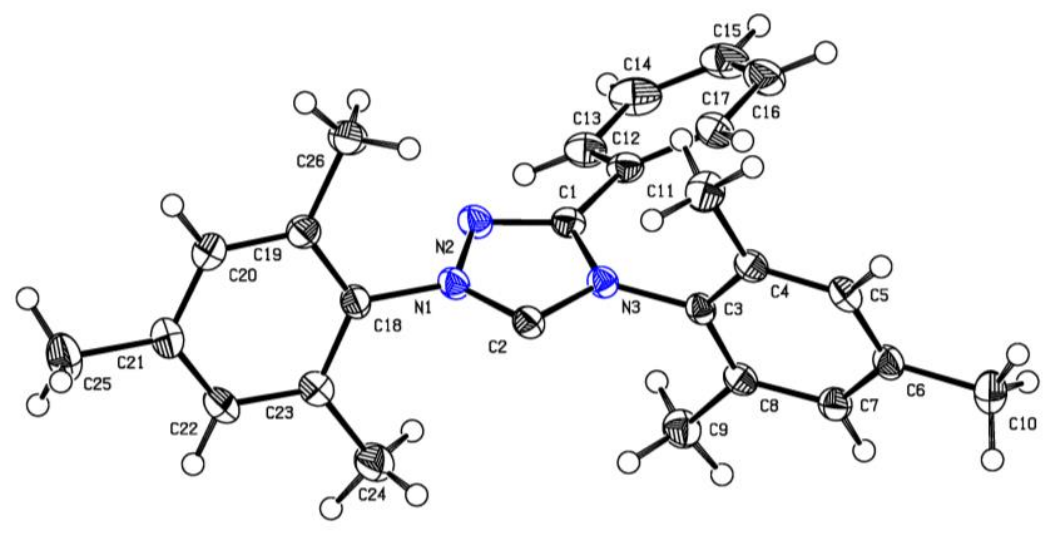

Figure S158. ORTEP of the X-ray crystal structure of 21a. Thermal ellipsoids are drawn at $50 \%$ probability level. 


\section{References}

[1] Enders, D.; Breuer, K.; Kallfass, U.; Balensiefer, T. Synthesis 2003, 1292-1295.

[2] (a) Rivas, F. M.; Riaz, U.; Giessert, A.; Smulik, J. A.; Diver, S. T. Org. Lett. 2001, 3, 2673-2676. (b) Rivas, F. M.; Giessert, A. J.; Diver, S. T. J. Org. Chem. 2002, 67, $1708-1711$.

[3] Bantreil, X.; Nolan, S. P. Nature Protocols 2011, 6, 69-77.

[4] Chow, K. Y.-K.; Bode, J. W. J. Am. Chem. Soc. 2004, 126, 8126-8127.

[5] Piel, I.; Pawelczyk, M.D.; Hirano, K.; Fröhlich, R.; Glorius, F. Eur. J. Org. Chem. 2011, 5475-5484.

[6] Li, P; Wu, C.; Zhao, J.; Rogness, D. C.; Shi, F.; J. Org. Chem. 2012, 77, 3149-3158.

[7] Pratapan, S.; Scaria, P. M.; Bhattacharyya, K.; Das, P. K.; George, M. V. J. Org. Chem. 1986, 51, 1972.

[8] Sibi, M. P.; Stanley, L. M.; Jasperse, C. P. J. Am. Chem. Soc. 2005, 127, 8276-8277.

[9] Ling, K. B.;. Smith, A. D. Chem. Commun. 2011, 47, 373-375.

[10] Wallasch, M. W.; Weismann, D.; Riehn, C.; Ambrus, S.; Wolmershäuser, G.; Lagutschenkov, A.; Niedner-Schatteburg, G.; Sitzmann, H. Organometallics 2010, 29, 806-813.

[11] Dijk, T. V.; Burck, S.; Rong, M. K.; Rosenthal, A. J.; Nieger, M.; Slootweg, J. C.; Lammertsma, K. Angew. Chem. Int. Ed. 2014, 53, 9068-9071.

[12] Frøseth, M.; Netland, K. A.; Rømming, C.; Tilset, M. J. Organomet. Chem. 2005, 690, 6125-6132.

[13] Li, Y.; Li, Z.; Xiong, T.; Zhang, Q.; Zhang, X. Org. Lett. 2012, 14, 3522-3525.

[14] Asselin, A. A.; Humber, L. G.; Dobson, T. A.; Komlossy, J.; Martel, R.R. J. Med. Chem. 1976, 19, 787-792.

[15] Tsang, W.C.P.; Munday, R. H.; Brasche, G.; Zheng, N.;. Buchwald, S. L. J. Org. Chem. 2008, 73, 7603-7610.

[16] Graebe, C.; Rateanu, A. Sc. Justus Liebigs Annalen der Chemie.1894, 279, 257-267.

[17] Struble, J. R.; Bode, J. W. Org. Synth. 2010, 87, 362-376.

[18] Zhang, L.; Sun, L.; Li, Y.-Y.; Liu, Y.; Yang, Y.-X.; Yuan, R.; Wang, P.; Da, C.-S. ChemCatChem 2013, 5, 3516-3519.

[19] Kiyooka, S.; Hena, M. A. J. Org. Chem. 1999, 64, 5511-5523.

[20] Fan, X.; Lv, H.; Guan, Y.-H.; Zhu, H.-B.; Cui, X.-M.; Guo, K. Chem. Commun. 2014, 50, 4119-4122.

[21] Bharathi, P.; Periasamy, M. Org. Lett. 1999, 1, 857-859.

[22] Wang, M. H.; Cohen, D. T.; Schwamb, C. B.; Mishra, R. K.; Scheidt, K. A. J. Am. Chem. Soc. 2015, 137, 5891-5894.

[23] Harit, U.; Rovis, T. J. Am. Chem. Soc. 2010, 132, 2860-2861. 
[24] Geu-Flores, F.; Sherden, N. H.; Courdavault, V.; Burlat, V.; Glenn, W. S.; Wu, C.; Nims, E.; Cui, Y.; O'Connor, S. E. Nature 2012, 492, 138-142.

[25] Erkkila, A.; Pihko, P. M.; J. Org. Chem. 2006, 71, 2538-2541. 